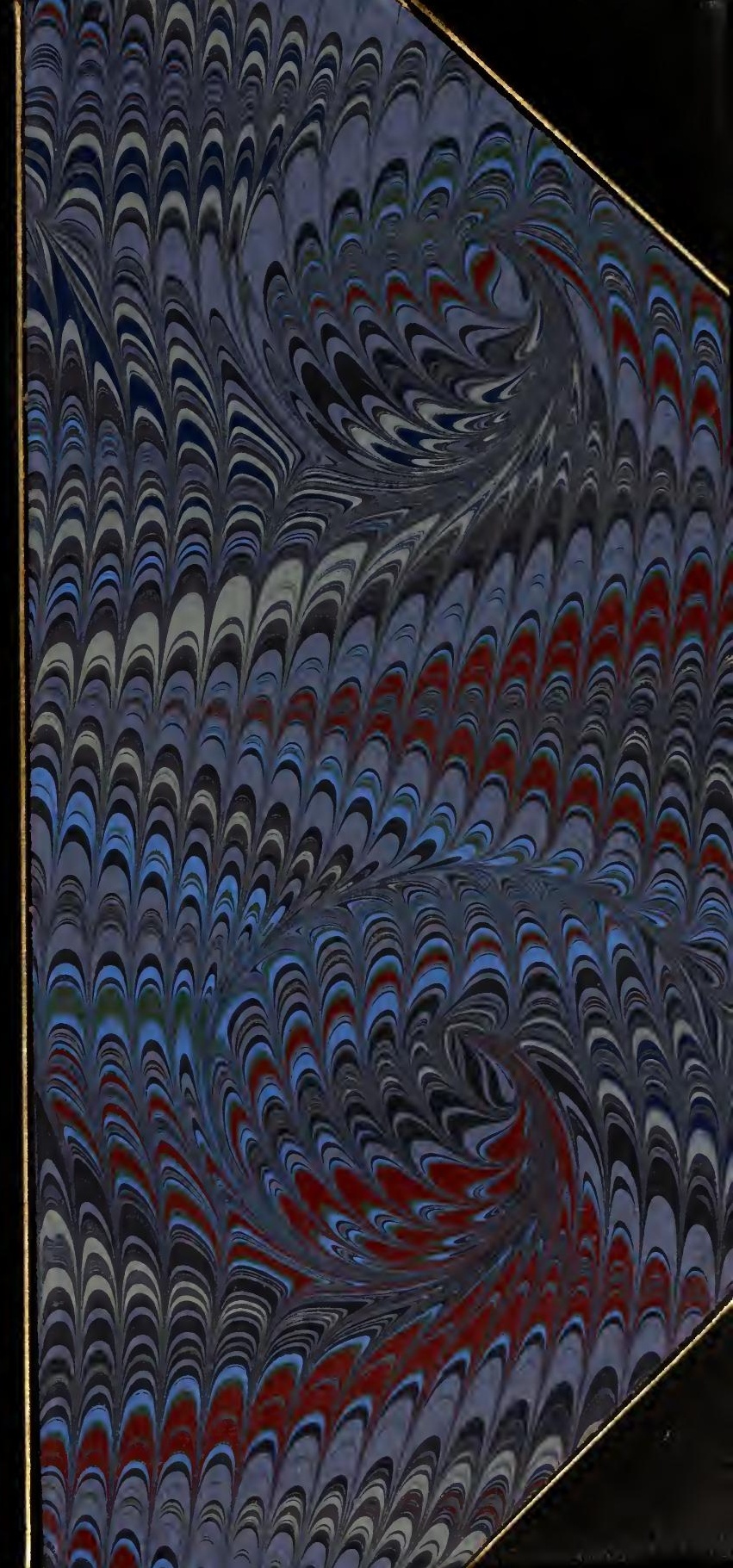








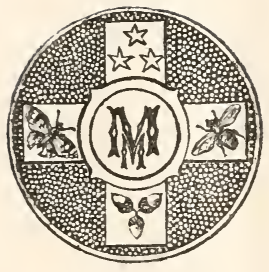




\section{THE}

\section{ELEMENTS OF EMBRYOLOGY.}

$\mathrm{BY}$

M. FOSTER, M.A., M.D., F.R.S.,

FELLOW OF AND PRELECTOR IN PHYSIOLOGY IN TRINITY COLLEGE, CAMBRIDGE,

AND

FRANCIS M. BALFOUR, B.A.,

FELLOW OF TRINITT COLLEGE, CAMBRIDGE.

Zlonion:

MACMILLAN AND CO.

I 874 .

[All Rights reserved.] 
Cambridge:

PBINTED BY C, J. CLAY, M.A. AT THE UNIVERSITY PRESS. 


\title{
THOMAS HENRY HUXLEY
}

\author{
AS A LITTLE TOKEN
}

OE OLR APPRECIATION OF HIS WORTII

\author{
AND OF
}

IIS MUCH KINDNESS TO OLRSELVES

\section{THIS BOOK}

IS RESPECTFCLLX DEDICATED BI

THE ACTHORS. 



\section{PREFACE.}

Is this volume we offer to the public the first part of what we hope may serve as a systematic introduction to the study of Embryology. Some apology is perhaps necessary for the separate publication of a part only of the whole subject; but we trust that the following reasons will justify the course we have adopted.

Those who have paid attention to recent embryological researches must be aware of what we may venture to call the tumultuous condition of many parts of the subject, and of the extreme difficulty in many cases of forming a clear and decided judgment without the aid of independent observations. It is this necessity of having repeatedly to work over contested points with a view to reconcile diametrically opposed statements, or to verify startling announcements, which has rendered so laborious the task we have undertaken, and which so much delays its completion.

On the other hand, whoever wishes to have a sound foundation of embryological knowledge cannot do better than gain a thorough insight into the development of the bird. The practical advantages offered by the hen's egg 
altogether outweigh the theoretical oljections to beginning with the avian type. In many respects, it might be thought desirable to commence with a holoblastic ovum; but the large food-yolk of the bird's egg is in many ways a great assistance to the study of changes going on in the blastoderm. The chick is of all embryos the best to begin with; when its history has once been mastered, the subsequent study of other forms becomes an easy matter.

We venture to hope therefore that we shall meet with general approval, in having described at considerable length the history of the chick, and in hastening the publication of our account, by bringing it forward in a separate form.

In the earlier chapters, especially, we have gone into very considerable detail; and in order to make the account intelligible to the beginner, have not been deterred by the fear of wearying our readers with elementary and recapitulatory statements. Debated matters and details of minor importance have been put in small print; these may be omitted by the student in reading the book for the first time. Though we have sometimes introduced names in connection with important observations, we have not thought it necessary to do this systematically. For recent or debated statements however, the authorities are always cited.

The worth of such a book as this will be very small if the student simply contents himself with reading what is written; and to facilitate the only really useful mode of study, that of actual observation, a few practical instructions have been added in an appendix.

The readiness with which the development of the skull can be studied in the chick renders it, in spite of obvious 
objections, a suitable introduction to the important subject of cranial morphology. It is with this view that we have given a separate chapter on the skull, which we hope may serve as an introduction to the study of Mr Parker's elaborate memoirs.

In the remaining parts, which we shall do our best to complete as soon as possible, the several histories will be treated with much greater brevity, and much more space will be given to theoretical considerations.

The figures, whose source is not acknowledged in the text, were drawn by Miss A. B. Balfour, except a few by ourselves.

The drawing on wood was executed partly by Mr Allchin, but chiefly by Mr Collings; and all the drawings were cut by Mr Cooper. We have to thank those gentlemen for the trouble they have taken in a matter in which, for many reasons, the result never seems commensurate with the labour. We are much indebted to Professor Huxley for having kindly looked over the proofs of the Chapter on the Skull.

The work took its origin in a course of lectures delivered by myself, but many causes prevented my taking the task seriously in hand, until I was joined by my friend and former pupil Mr F. MI. Balfour, whose share in the matter has, to say the least, been no less than my own.

\section{FOSTER.}





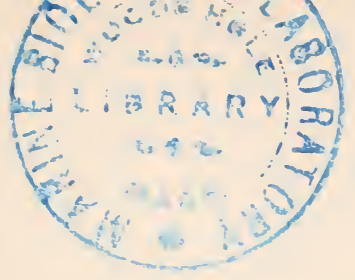

\section{TABLE OF CONTENTS.}

INTRODUCTION • • • • • • • • pp. I-IO.

\section{PART I. THE HISTORY OF THE CHICK.}

\section{CHAPTER I.}

The Structure of the Hen's Egg, and the Chaxges which take place UP to the BeginNixg of INcUbation • . pp. II-26.

I. The shell. 2. The shell-membrane. 3. The albumen. 4. The vitelline membrane. 5. The yolk. 6. The yellow yolk. 7 . The white yolk. 8. The white yolk-spheres. 9. The structure of the blastoderm. Io. Recapitulation. II. The ovarian ovum. I2. The descent of the ovum along the oviduct. I3. The impregnation of the ovum. I4. Segmentation. I5. The formation of the upper and lower layers.

\section{CHAPTER II.}

A Brief Sedrary of the whole History of Incubation, pp. 2ろ-42.

I. The embryo is formed in the area pellucida. 2. The epiblast, mesoblast, and hypoblast. 3. The extension of the blastoderm over the yolk. 4. The vascular area. 5. The head-fold and the other folds by means of which the embryonic sac is formed. 6. The outward shape of the embryo. 7. The formation of the neural tube and alimentary canal: somatopleure and splanchnopleure. 8. The amnion. 9. The allantois.

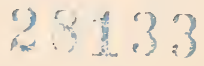




\section{CHAP'TER III.}

The Chajges whici make place during the Frost Day of Incubation, pp. $43-57$.

I. Variations in the progress of development. 2. The embryonic shield. 3. The formation of the epiblast, mesoblast and hypoblast. 4. The primitive streak, the primitive groove. 5. The head-fold, the medullary groove, meclullary folds, and notochord. 6 . The amnion; the changes taking place in the three layers. 7. The increase of the head-fold. 8 . The closure of the medullary canal. 9, ro. The cleavage of the mesoblast: formation of splanchnopleure and somatopleure. II. The protovertebræ. I2. The formation of the vascular area. I3. Recapitulation.

\section{CHAPTER IV.}

The Changes whica take place during the Second Day, pp. $58-83$.

1. Increasing distinctness and prominence of the embryo. 2. The first cerebral vesicle. 3. The increase in the number of protovertebræ. 4. The first rudiments of the alimentary canal. 5. The formation of the heart. 6 . The formation of blood-vessels; the omplalo-mesaraic veins and arteries, the sinus terminalis. 7 . Changes taking place in the cells of the several layers. 8. The rudiment of the Wolffian duct. 9. Recapitulation of the changes during the first half of the second day. Io. Increasing prominence of the embryo; the tail-fold and the lateral folds. II. Continued closure of the medullary canal. I2. The optic vesicles. I3. The second and third cerebral vesicles. I 4. Change of position of the optic vesicles. I5. The vesicles of the cerebral hemispheres. I6. The cranial flexure. I 7 . The rudinent of the ear, or auditory sac. I8. Changes in the heart. 19. The primitive aortæ and first pair of aortic arches, the omphalo-mesaraic vessels, the sinus terminalis. 20. The second and third pair of aortic arches. 2I. The Wolffian duct. 22. The amnion. 23. Recapitulation.

\section{CHAPTER V.}

\section{The Chayges which take place during the Third Day, pp. $S_{4}$-I 40 .}

x. The diminution of the albumen. 2. The spreading of the opaque and vascular areas. 3. The vascular area. 4. The continued folding in of the embryo. 5. The increase of the amnion. 6. The change in the position of the embryo. 7. The curvature of the body. 8. The cranial flexure. 9. Growth of the vesicles of the cerebral hemispheres; the third ventricle, pineal gland, infundibulum and pituitary body, the cerebellum and medulla oblongata. Io. Changes in the spinal cord. II. The formation of the eye. Histological changes in the retina, optic nerve, and lens. I2. The formation of the ear. I3. The nasal pits. I4. The visceral ciefts and folds. I5. The aortic arches. 16 . Changes in the heart; the Ductus Cuvieri and cardinal veins. ${ }^{1} \tau$. The folding in of the alimentary canal; the formation of the tail. 18. The lungs. 19. The liver. 20. The pancreas and spleen. 21. The thyroid body. 22. Changes in the trunk of the embryo. 23. Separation of the muscle-plates from the protovertebræ. 24. Growth of the intermediate cell-mass. 25. The cranial nerves. 26. The Wolffian duct. 27. Recapitulation. 


\section{CHAPTER VI.}

The Changes which take place durixg the Fourth Dar, pp. $141-173$.

r. Appearances on opening the egg. 2. Growth of the amnion. 3. Narrowing of the splanchnic stalk. 4. Increase in the cranial flexure. 5. The first appearance of the limbs. 6. Growth of the head. 7. Changes in the nasal pits. 8. Formation of the mouth. 9. The cranial nerves. I0. The allantois. $\mathbf{I}_{\bar{\imath}}$. Changes in the protovertebræ; the spinal ganglia. I2. The secondary segmentation of the vertebral column. 13. Changes in the notochord. I4. Ossification of the vertebræ. I5. The ribs. I6. Changes in the muscle-plates. I7. The Wolffian body and duct. I8. The duct of Müller. I9. The kidneys. 20. The ovaries and testes. 21. Changes in the arterial system. 22. Changes in the venous system; the veins of the liver. 23. Changes in the heart; the ventricular septum. 24. Recapitulation.

\section{CHAPTER VII.}

The Chaxges which take place ox the Fifth Dar, pp. I7千-I99.

I. Appearances on opening the egg. 2. The growth of the limbs. 3. The cranium; the investing mass and trabeculæ. 4. Changes in the face; formation of the nose and nasal passages. 5. Appearance of the anus. 6. Changes in the spinal cord; the formation of the grey and white columns, and of the posterior and anterior fissures. 7. Changes in the heart; the rudiment of the auricular septum, the division of the bulbus arteriosus into aorta and pulmonary artery, the formation of the semilunar valves. 8. Changes in the heart during the sixth day. 9. Subsequent changes in the heart; the completion of the auricular septum, the arrangement of the openings of the venæ cavæ. Io. Histological differentiation; the fate of the three primary layers. II. Recapitulation.

\section{CHAPTER VIII.}

Froy the Sixth Das to the end of Incebation, pp. 200-224.

I. The commencement of distinct avian differentiation. 2. The fœtal appendages during the sixth and seventh days. 3. During the eighth, ninth and tenth days. 4. From the eleventh to the sixteenth day. 5. From the sixteenth day onwards. 6 . The changes in the general form of the embryo during the sixth and seventh days. 7 . During the eighth, ninth and tenth days. 8. From the eleventh day onwards; feathers, ossifications. 9. Changes in the venous system before and after the commencement of pulmonary respiration. Io. Changes in the arterial system, the modifications of the aortic arches. II. Summary of the chief phases of the circulation. I2. Exclusion from the egg. 


\section{CHAPTER IX.}

\section{The Defelopaent of the Skull, pp. 225-23S.}

I, 2. The prirnordial cranium. 3, 4. The investing mass of Rathke. 5. The trabeculæ cranii. 6. The cartilages of the first visceral arch. 7 . The maxillary process. 8. The mandibular arch. 9. The hyoid arch. ro. The cartilages of the third visceral arch. J I. Changes in the cranium during the fifth and sixth days. I2. During and after the seventh day. 13. The condition of the cranium at about the middle of the second week. r+. Ectosteal and endosteal ossifications of the cartilaginous cranium. 15. Formation of the membrane bones. I6. Progress of ossification during the second and third weeks. I7. Fenestration of the ethmo-presphenoid cartilage: I8. Ossifications in the prootics and alisphenoid. I9. Changes in the basitemporals. Formation of the vomer. 20. The changes which take place immediately after exclusion from the egg. 2r. Further changes in the splint bones. Coalescence of the bones after birth. Table of bones classified according to their mode of ossification.

\section{APPENDIX.}

Practical Instructions for studying the Developjext of the Chick, Pp. $239-267$.

I. Incubators. II. Examination of a $3^{6}$ to $4^{8}$ hours embryo. III. Examination of an embryo of about $48-50$ hours. IV. Of an embryo at the end of the third day. V. Of an embryo of the fourth day. VI. Of a blastoderm of 20 hours. VII. Of an unincubated blastoderm. VIII. Of the process of segmentation. IX. Of the later changes of the embryo. $\mathrm{X}$. Study of the development of the blood-vessels.

\section{ERRATUM.}

p. $12_{4}$, in the description of Figr. $39 B$, for 'Superior vertebral' substitute 'Jugular.' 


\section{LIST OF ILLUSTRATIONS.}

FIG.

I. Dilgramatic Section of an Unincubated Fuwl's Egg . . .

2. A. Yellow yolk-sphere filled with fine granules. $B$. White yolkspheres and spherules of various sizes and presenting different appearances

3. Section of a Blastoderm of a Fowl's Egg at the commencement of Incubation . . . . . . . . . . .

4. Section through the Germinal Dise of the ripe Ovarian Ovum of a Forwl while yet enclosed in its Capsule. . . . . .

5. Surface Views of the early Stages of the Segmentation in a Fowl's Egg

6. Surface View of the Germinal Disc of a Hen's Egg during the later Stages of Segmentation

7. Section of the Germinal Disc of a Fowl during the later Stages of Segmentation

8. 4 to $N$. A series of purely diagrammatic representations introduced to facilitate the comprehension of the manner in which the body of the embryo is formed, and of the various relations of the yolk-sac, amnion and allantois . . . . . . 29-32

9. Diagrammatic Longitudinal Section through the Axis of an Embryo.

ro. Section of a Blastoderm at right angles to the long axis of the Embryo after eight hours' Incubation . 
FIG.

I I. Surface View of the Pellucid Area of a Blastoderm of 18 hours

1 2. Transverse Section of a Blastoderm incubated for 18 hours

13. Transverse Section through the Dorsal Region of an Embryo of the Second Day

14. An Embryo Chick of the First Day (about thirty-six hours) viewed from below as a transparent object

I5. Embryo of the Chick at $3^{6}$ hours viewed from alove as an opaque object.

:6. Diagrammatic Longitudinal Section through the Axis of an Embryo.

17. A, B. Two consecutive Sections of a $3^{6}$ hours Einbryo illustrating the formation of the heart

13. Transverse Section of an Embryo at the end of the Second Day passing through the region of bulbus arteriosus

19. Surface View from below of a small portion of the posterior end of the pellucid area of a 36 hours Chick

20. Transverse Section through the Dorsal Region of an Embryo of 4 ; hours.

21. Embryo of the Chick at 36 hours viewed from above as an Opaque Object

22. Head of a Chick at the End of the Second Day viewed from below as a Transparent Object

23. Diagram of the Circulation of the Yolk-Sac at the end of the Third Day of Incubation

24. Chick of the Third Day (5t hours) viewed from underneath as a Transparent Object

25. Head of a Chick of the Third Day viewed sideways as a Transparent Object

26. Section through the Hind-Brain of a Chick at the end of the Third Day of Incubation

27. Diagrammatic Sections illustrating the Formation of the Eye.

28. Diagrammatic Section of the Eye and the Optic Nerve at an early stage . 
FIG.

PAGE

29. Diagrammatic representation of the Eye of the Click of about the Third Day as seen when the head is viewed from underneath as a transparent object

30. D, E, F. Diagrammatic Sections of the Eye of the Chick of about the Third Day.

31. Section of the Eye of Chick at the Fourth Day

100

32. Section through the Hind-Brain of a Chick at the end of the Third Day of Incubation

33. Two Views of the membranous Labyrinth of Columba domestica. A from the exterior, $\mathrm{B}$ from the interior

I I 2

34. Transverse Section of the Head of a Fœtal Sheep $(16 \mathrm{~mm}$. in length) in the region of the Hind-Brain

35. Section of the Head of a Fœtal Sheep ( $20 \mathrm{~mm}$. in length)

I I +

35. Section through the internal Ear of an Embryonic Sheep (28 mm. in length) .

37. Head of an Embryo Chick of the Third Day viewed siderays as an Opaque Object

3S. The same Head as shewn in Fig. 37 , seen from the Front

39 A. Diagram of the Arterial Circulation on the Third Day .

I 22

39 B. Diagram of the Venous Circulation on the Third Day

$12+$

40. Section of the Tail-end of an Embryo (Chick) of the Third Day

125

fr. Section through the Dorsal Region of an Embryo at the commencement of the Third Day

42. Diagram of a portion of the Digestive Tract of a Chick upon the Fourth Day.

43. Four diagrams illustrating the Formation of the Lungs .

44. Section through the Dorsal Region of an Embryo at the end of the Third Day

45. Head of an Embryo Chick of the Third Day (seventy-five hours) viewed sideways as a transparent object

46. Embryo at the end of the Fourth Day seen as a transparent object. 
FIG.

47. Section through the Lumbar Region of an Embryo at the End of the Fourth Day .

48. A. Head of an Embryo Chick of the Fourth Day viewed from below as an opaque object. B. The same seen sideways . . $\mathbf{r}_{4} 6$

49. Longitudinal Section of the Tail-end of an Embryo Chick at the commencement of the Third Day

50. Longitudinal Section of the Tail-end of an Embryo Chick at the middle of the Third Day

5r. Section of the intermediate Cell-mass on the Fourth Day

52. State of Arterial Circulation on the Fifth or Sixth Day .

$\mathrm{I}_{4} \mathrm{~S}$

53. Diagram of the Venous Circulation at the Commencement of the Fifth Day .

54. Heart of a Chick on the Fourth Day of Incubation viewed from the Ventral Surface.

55. View from above of the Investing Mass and of the Trabecule on the Fourth Day of Incubation

56. A. Head of an Embryo Chick of the Fourth Day viewed from below as an opaque object. B. The same seen sideways. . r\&o

57. Head of a Chick at the Sixth Day from below . . . . I8I

58. Head of a Chick of the Seventh Day from below . . . . I82

59. Section through the Spinal Cord of a Seven Days Chick . . r8s

6. Two views of the Heart of a Chick upon the Fifth Day of Incubation . . . . . . . . . . . . 192

Gr. Heart of a Chick upon the Sixth Day of Incubation, from the Ventral Surface.

62. Diagram of the Venous Circulation at the Commencement of the Fifth Day .

63. Diagram of the Venous Circulation during the later days of Incubation

64. Diagram of the Venous Circulation of the Chick after the commencement of Respiration by means of the Lungs . . . 2 II

65. State of Arterial Circulation on the Fifth or Sixth Day . . . 212 
66. Diagram of the Condition of the Arches of the Aorta towards the Close of Incubation . . . . . . . . 2 I6

67. Diagram of the Arterial System of the Adult Fowl . . 2 29

68. View from above of the Investing Mass and of the Trabeculæ on the Fourth Day of Incubation . • • . • . 226

69. View from below of the Paired Appendages of the Skull of a Fowl on the Fourth Day of Incubation . . . . . 229

70. Side view of the Cartilaginous Cranium of a Fowl on the Seventh Day of Incubation . . . . . . . . . 231

7r. Embryonic Skull of a Fowl during the Second TVeek of Incubation (third stage) from below . . . . . . . . 234 



\section{INTRODUCTION.}

EvEry living being passes in the course of its life through a series of changes of shape and structure. These changes may, in their completest form, be considered as constituting a morphological cycle, beginning with the ovum and ending with the ovum again.

Among many living beings and especially among vertebrate animals by far by the greater part of the life of the individual is spent in one particular phase, which is not only of longer duration than the rest, but also of much more importance, inasmuch as during it the greater part of the 'work' of the living being is done. This is generally spoken of as the adult stage, and in most cases immediately precedes, or is peculiarly associated with, the completion of the morphological cycle in the appearance of a new ovum.

The word embryology may be generally taken to mean the study of the successive morphological phases through which a living being passes from the ovum to the adult stage, or the study of the gradual 'development' of the ovum to the adult form ; though, especially among some of the socalled lower forms of life, its meaning must be so extended as to embrace all the morphological phases of an individual life. Embryology is thus a part of and a necessary introduction to the wider study of 'Generation.' As a matter of 
history we find that the study of it sprang out of the various attempts to solve the problems of why and how living beings come into existence.

It would be beyond the scope of this work to enter at all fully into any account of the earlier of these inquiries from those of Aristotle downwards; but it may be of some use to point out the chief steps by which in modern times embryology has been established as a distinct branch of knowledge.

From the very first, incubated bird's eggs, and especially hen's eggs, owing to their abundance at all seasons, and the ease with which they could be examined, became special objects of study. Aristotle examined the growing chick within the egg, and gave the name of punctum saliens to the 'bloody palpitating point,' which marks the growing heart in the early days of incubation. Since his time all observers have had recourse to the hen's egg; and though it may be urged that the highly specialised characters of the avian type unfit it for so general a purpose as that of serving as the foundation of embryology, the practical advantages of the bird's egg over either the mammalian or any other ovum, are so many, that it must always continue to be, as it has been, a chief object of study.

From the time of Aristotle down to that of Fabricius of Aquapendente so little progress in real observation of facts had been made, that we find the latter anatomist (De Formatione Ovi et Pulli, 1621) describing the chick as being formed out of the chalaze of the white of the egg; a view which lived long afterwards, and whose influence may still be recognized in the names 'tread' or 'treadle' which the housewife sometimes gives to those portions of thickened albumen.

Harvey was the first to clearly establish that the essential part of the hen's egg, that out of which the embryo pro- 
ceeded, was the cicatricula. This Fabricius had looked upon as a blemish, a scar left by a broken peduncle. In his Anatomical Exercises on the Generation of Animals (1651), Harvey describes the little cicatricula as expanding under the influence of incubation into a wider structure, which he calls the eye of the egg; and at the same time separating into a colliquamentum. In this colliquamentum, according to him, there appears, as the first rudiment of the embryo, the heart or punctum saliens, together with the blood-ressels. These gradually gather round them the solid parts of the body of the chick. Harvey clearly was of opinion that the embryo arose, by the successive formation of parts, out of the homogeneous nearly liquid colliquamentum. He was an early advocate of the doctrine of epigenesis.

'Notwithstanding the weight of Harvey's authority, the doctrine of epigenesis subsequently gave way to that of evolution, according to which the embryo pre-existed, eren though invisible, in the ovum, and the changes which took place during incubation consisted not in a formation of parts, but in a growth, i.e. in an expansion with concomitant changes, of the already existing germ. Of this theory Malpighi is frequently said to have been the founder. In a limited sense this is true. In his letter to the Royal Society of London, De Formatione Pulli in Ovo (1672), he confesses himself compelled to admit that even in unincubated eggs an embryo was present (Quare pulli stamina in ovo pre-existere, altioremque originem nacta esse fateri convenit). Yet he evidently struggled against such a conclusion, and instead of developing a consistent theory of evolution, left the earliest stages of the embryo as too mysterious to be profitable objects of study, and contented himself with tracing out the events of later lays. From his descriptions it is clear that his so-called unincubated egrs 
had under the warmth of summer already made considerable progress in development.

The man who first logically worked out a theory of evolution and became its most distinguished and zealous adrocate was Haller (Sur la Formation du Cour dans le Poulet, 1758, and Elementa Physiologice, Liber xxix. 1766).

This great anatomist insisted that the embryo existed even in the unincubated egg though in a rudimentary form, and indeed invisible. He supposed that it was a vermiform structure composed of all the essential parts of a full-grown animal in an undeveloped state, and that the effect of incubation was to educe or evolve these undeveloped organs into an adult condition. The same views were urged with characteristic extravagance by Bonnet (Considérations sur les corps organisés, 1762).

'This doctrine of evolution or prædilineation, as it was called at the time, was doomed to be overthrown even in Haller's own day.

In an inaugural dissertation entitled Theoria Generationis, published 1759, Casper Frederick Wolff laid the foundations of not only modern Embryology, but modern Histology. He shewed that the cicatricula of the unincubated hen's egg consisted of a congeries of particles (such as we now call cells) all alike, or divisible into groups only, and that anything like distinct rudiments of an embryo were wholly absent. Out of these particles the embryo was built up by means of a series of successive changes (several of which he described in detail, especially in his work on the Formation of the Alimentary (anal, 1768), part being added to part, and parts once formed being modified into fresh parts. Thus the old imperfect theory of evolution was supplanted by a view, which, under the term of epigenesis, was in reality a more complete and truer theory of evolution. Wolff also shewed that all the parts as well of plants as of animals could be conceived of 
as being arrangements of these particles or cells variously modified, and that all the phenomena of the form and structure of living beings were to be regarded as the results of a variable nutritive energy, to which he gave the name vis essentialis.

Haller complained of Wolff, that he had attempted to make a great leap instead of being contented with small onward steps. Wolff's leap proved too great for his time. While his insight into the fundamental doctrines of histology remained for the most part without fruit till the next century, so also the way he opened up in embryology was successfully followed by no one for many years after.

In 1816 that admirable teacher Döllinger, of Würzburg, induced Pander to take up the study of the incubated hen's egg. We owe to Pander (Dissertatio Inauguralis sistens Historiam Metamorphoseos quam Ovum Incubatum prioribus quinque diebus subit, and Beiträge zur Entwickelungsgeschichte des Hühnchens im Eie) a clear and excellent description of many of the changes which take place during the early days of incubation. It was he who introduced the term blastoderm. He too first drew attention to the distinction of the three layers, serous, mucous, and vascular. But his greatest merit perhaps consisted in the fact of his studies having been the exciting cause of those of Von Baer.

Coming to Würzburg to study under Döllinger, and finding Pander busily engaged in his embryological work, Von Baer enthusiastically took up the same subject, and thenceforward devoted the greater part of his life to it.

Of the results of his labours, which are embodied in his Entwickelungsgeschichte der Thiere, 1828, 1837, this simply may be said. Von Baer found the true line of inquiry already marked out by Wolff. He followed up that line so sedulously and with such success, that nearly all the work which has been done since his day up to the present time, in Vertebrate 
Embryology, may be regarded as little more than an extension, with corrections, of his observations. Were it desirable to re-publish Von Baer's work, the corrections and expansions of matters of fact necessary to bring it up to the present time, as the phrase goes, would, with some few exceptions, be of minor importance, though they might be many. The theoretical considerations embodied in his Scholia through which he interprets the morphological significance of embryological facts are of great and lasting importance, though they need some modifications in order to bring them into harmony with the theory of natural selection. Since Von Baer's time, the advances made in Vertebrate Embryology, through the elaborate work of Remak, the labours of Rathke, Allen Thomson and others, the admirable lectures of Kölliker, and the researches of more recent inquirers, though many and varied, cannot be said to constitute any epochs in the history of the subject, such as that which was marked by Von Baer, and before him by Wolff. We may perhaps make an exception in favour of the discovery by Purkinje, of the germinal vesicle in the fowl's ovarian ovum (1825). This led to Von Baer's discovery of the mammalian ovum (1827), which first rendered possible a consistent view of mammalian generation.

The study of invertebrate embryology has, on the other hand, during the last few years produced the most striking results.

In the following pages we propose to follow in the path thus marked out by the history of the subject. We begin with the chick as being the animal which has been most studied, and the study of which is easiest, and most fruitful for the beginner. The first part accordingly will be devoted to a description of the changes undergone by an incubated hen's egg, especially during the early days of incubation. We shall endeavour to explain, with such details as are necessary, 
the manner in which the embryo is formed, and the way in which the rudiments of the most important organs of the chick arise. We shall follow a chronological order, tracing out the changes day by day (or with even shorter periods), during the first few days. We are convinced that this method (adopted by Von Baer) is on the whole the one which most commends itself to the learner. It has of course its disadvantages; and in several instances we have found it desirable when describing, at its appropriate date, the most striking phase in the development of an organ, at once to follow up the subsequent history, instead of giving it piecemeal afterwards. But the general advantages of the chronological method, especially when the reading of such a book as this is rendered really useful by an accompanying actual examination of incubated eggs, are so great that they far outweigh the evil of any such slight irregularities. After tracing out the history of the several organs, no farther than is necessary to give a clear idea of the general course of events in each case, we propose to treat the changes and incidents of the latter days of incubation with great brevity, not attempting any special account of avian development, except in the case of the skull. And even this will be treated summarily. The First Part will therefore really be an introduction to the general facts of vertebrate embryology, the chick being taken as an example.

In the Second Part we purpose to consider the embryonic histories of other vertebrates, in so far as these differ from that of the bird; and then to treat of the development of special organs in a more complete manner.

The Third Part will be devoted to an exposition of the main facts of invertebrate embryology, and to the discussion of general morphological considerations. 
The reader will scarcely fail to notice that the First Part especially is entirely confined to a simple description of observed facts, no attempt whatever being made to interpret their meanings. We have purposely pursued this course, because any interpretation of the facts of the bird's development is impossible, or at least illusory, till the history of other animals, vertebrate and invertebrate, has been studied. When all the facts are before him the reader will be in a position to judge of the interpretations offered. 


\section{PART I.}

\section{THE HISTORY OF THE CHICK.}





\section{CHAPTER I.}

THE STRUCTURE OF THE HEN'S EGG, AND THE CHANGES WHICH TAKE PLACE UP TO THE BEGINNING OF INCUBATION.

1. Ix a hen's egg quite newly laid we meet with the following structures. Most external is the shell (Fig. 1, s.), composed of an organic basis, impregnated with calcic salts. It is sufficiently porous to allow of the interchange of gases between its interior and the external air, and thus the chemical processes of respiration, feeble at first, but gradually increasing in intensity, are carried on during the whole period of incubation.

According to Nathusius, Zeitsch. f. Wiss. Zool. Vol. xriIr. p. 225-270, XIX. $322-348$, XX. IO6-I 20 , XXI. 330-355, the egg-shell of birds consists of an outer thimner and an inner thicker layer. The outer layer varies considerably in its consistency in different species. It is soft and pliant in the hen, but in many other birds, as for instance the ostrich, is hard and friable. It is frequently striated hoth vertically and transversely. Pigment when present is confined to this layer. The inner layer is thicker; and its interual surface is marked with rounded processes more or less separated from one another, whose blunt extremities are sunk into the shell-membrane. The presence of these processes must be considered as universal anongst birds. Vertical sections shew that this layer is composed of alternating horizontal laminæ of transparent and opaque material, the opaque laminæ being composed of exceedingly minute particles of an organic nature imbedded in a matrix impregnated with calcic salts.

Both layers of the shell are pierced by vertical canals, which are simple in Carinate but ramified in Ratite birds. These canals open freely on the exterior surface and also on the interior surface in the pits between the blunt processes of the inner layer. It is probable that the outer openings of these canals become closed by the presence of moisture, so that when the shell is wet neither air nor water can pass through it. If the shell is dry, air will penetrate easily; and if the upper layer with the free ends of the tubes be rubbed off, both water and air will pass through it without difficulty. In eggs with coloured shells the colouring matter frequently passes into the canals. 
2. Lining the shell, is the shell-membrane, which is double, being made up of two layers; an outer thicker (Fig. 1, s. m.), and an inner thinner one (i.s. m.). Both of these layers consist of several laminæ of felted fibres of various sizes, intermediate in nature between connective and elastic tibres.

FIG. I.

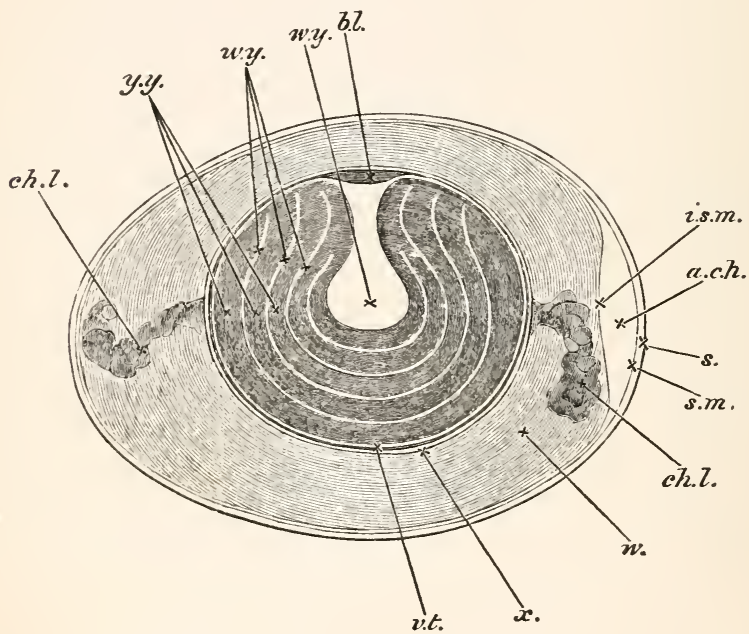

Diagrammatic Section of an Unincobated Fowl's Egg (modified from Allen Thomson).

ul. blastoderm. $w . y$. white yolk. This consists of a central flask-shaped mass and a number of layers concentrically arranged around this. $y$. $y$. yellow yolk. $v$. $t$. vitelline membrane. $x$. layer of more fluid albumen immediately surrounding the yolk. $w$. albumen consisting of alternate denser and more fluid layers. ch.l. chalaza. a. ch. air-chamber at the broal end of the egg. This chamber is merely a space left between the two layers of the shell-membrane. $i . s . m$. internal layer of shellmembrane. s. $m$. external layer of shell-membrane. s. shell.

Over the greater part of the egg the two layers of the shell-membrane remain permanently in close apposition to each other; but at the broad end they tend to separate, and thus to develope between them a space into which air finds its way. This air-chamber, as it is called, is not to be found in perfectly fresh eggs, but makes its appearance in 
eggs which have been kept for some time, whether incubated or not, and gradually increases in size, as the white of the egg shrinks in bulk by evaporation.

3. Immediately beneath the shell-membrane is the white of the egg or albumen (Fig. 1, w.), which is, chemically speaking, a mixture of various forms of proteid material, with fatty, extractive, and saline bodies.

Its average composition may be taken as

I $2 \circ$ p. c. proteid matter,

I.5 p. c. fat and extractives,

$\because$ p. c. saline matter, chiefly sodic and potassic chlorides, with phos. 86.0 p. c. water. phates and sulphates,

The white of the egg when boiled shews in section alternate concentric layers of a transparent and of a finely granular opaque material. In the natural condition, the layers corresponding to these opaque layers are composed of more fluid albumen, while those corresponding to the transparent layers are less fluid, and consist of networks of fibres, containing fluid in their meshes. The outer part of the white, especially in eggs which are not perfectly fresh, is more fluid than that nearer the yolk. The innermost layer, however, immediately surrounding the yolk (Fig. $1, x$.), is of the more fluid finely granular kind.

In eggs which have been hardened a spiral arrangement of the white may be observed, and it is possible to tear off laminæ in a spiral direction from left to right, from the broad to the narrow end of the egg.

Two twisted cords called the Chalazce (Fig. 1, ch. l.), composed of coiled membranous layers of the less fluid albumen, run from the two extremities of the egg to the opposite portions of the yolk. Their inner extremities expand and merge into the layer of denser albumen surrounding the Huid layer next the yolk. Their outer extremities are free, and do not quite reach the outer layer of the white. Thus they cannot serve to suspend the yolk, although they may help to keep it in position, by acting as elastic pads. The interior of each chalaza presents the appearance of a succession of opaque white knots; hence the name chalazæ, grandines (hailstones).

4. The yolk is enclosed in the vitelline membrane (Fig. 1, v. t.), a transparent somewhat elastic membrane easily 
thrown into creases and wrinkles. It might almost be called structureless, but under a high power a fine fibrillation is visible, and a transverse section has a dotted or punctated appearance; it is probably therefore composed of fibres. Its affinities are with elastic rather than connective tissue.

The vitelline membrane of most vertebrates is perforated by fine pores. These are largest in osseous fishes and much finer in mammals; they have not been found in the vitelline membrane of birds.

5. The whole space within the vitelline membrane is occupied by the yolk. To the naked eye this appears tolerably uniform throughout, except at one particular point of its surface, at which may be seen, lying immediately under the vitelline membrane, a small white disc, about $4 \mathrm{~mm}$. in diameter. This is the blastoderm, or cicatricula.

A tolerably typical cicatricula in a fecundated egg will shew an outer white rim of some little breadth, and within that a circular transparent area, in the centre of which, again, there is an opacity, varying in appearance, sometimes uniform, and sometimes dotted.

The dise is always found to be uppermost whatever be the position of the egg, provided there is no restraint to the rotation of the yolk. The explanation of this is to be sought for in the lighter specific gravity of that portion of the yolk which is in the neighbourhood of the disc, and the phenomenon is not in any way due to the action of the chalazæ.

A section of the yolk of a hard-boiled egg will shew that it is not perfectly uniform throughout, but that there is a portion of it having the form of a flusk, with a funnelshaped neck, which, when the egg is boiled, does not become so solid as the rest of the yolk, but remains more or less fluid.

The expanded neck of this flask-shaped space is situated immediately underneath the disc, while its bulbotis enlargement is about the middle of the yolk. We shall return to it directly.

6. The great mass of the yolk is composed of what is known as the yellow yolk (Fig. 1, y. y.). This consists of spheres (Fig. 2, A.) of from $25 \mu$ to $100 \mu^{1}$ in diameter, never containing a nucleus, but filled with numerous minute highly refractive granules; these spheres are very delicate and easily

$$
{ }^{1} \mu=.001 \mathrm{~mm} \text {. }
$$


destroyed by crushing. When boiled or otherwise hardened in situ, they assume a polyhedral form, from mutual pressure. The granules they contain seem to be of an albuminous nature, as they are insoluble in ether or alcohol.

Chemically speaking the yolk is characterized by the presence in large quantities of a proteid matter, having many affinities with globulin, and called vitellin. This exists in peculiar association with the remarkable body Lecithin. (Compare Hoppe-Seyler, Hdb. Phys. Chem. Anal.) Other fatty bodies, colouring matters, extractives (and, according to Dareste, starch in small quantities), \&c. are also present. Miescher (Hoppe-Seyler, Chem. Untersuch. p. 502) states that a considerable quantity of nuclein may be obtained from the yolk, probably from the spherules of the white yolk.

FIG. 2.
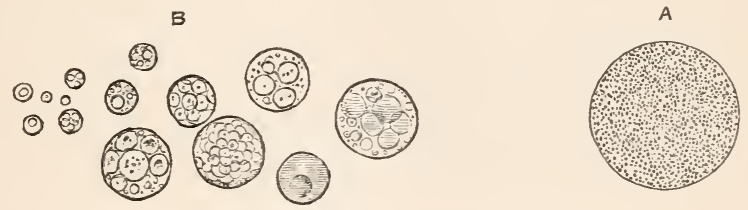

A. Yellow yolk-sphere filled with fine granules. The outline of the sphere has been rendered too bold.

$B$. White yolk-spheres and spherules of various sizes and presenting different appearances. (It is very difficult in a woodcut to give a satisfactory representation of these peculiar structures.)

7. The yellow yolk thus forming the great mass of the entire yolk is clothed externally by a thin layer of a different material, known as the white yolk, which at the edge of the blastoderm passes underneath the disc, and becoming thicker at this spot forms, as it were, a bed on which the blastoderm rests. Immediately under the middle of the blastoderm this bed of white yolk is connected, by a narrow neck, with a central mass of similar material, lying in the middle of the yolk (Fig. 1, w. y.). When boiled, or otherwise hardened, the white yolk does not become so solid as the yellow yolk; hence the appearances to be seen in sections of the hardened yolk. The upper expanded extremity of this neck of white yolk is generally known as the "nucleus of Pander."

Concentric to the outer enveloping layer of white yolk there are within the yolk other inner layers of the same substance, which cause sections of the hardened yolk to 
appear to be composed of alternate concentric thicker laminæ of darker (yellow) yolk, and thinner laminæ of lighter (white) yolk (Fig. 1, $w, y$.).

S. The microscopical characters of the white yolk are very different from those of the yellow yolk. It is composed of spheres (Fig. 2, B.) for the most part smaller than those of the yellow yolk $(4 \mu-75 \mu)$, with a highly refractive nucleuslike body often as small as $1 \mu$ in the interior of each; and also of larger spheres, each of which contains a number of spherules, similar to the smaller spheres; these latter appearing to have passed into the larger spheres, by a process of inclusion.

There has been a considerable amount of controversy as to whether these elements possess a membrane; there is little doubt however that there is no membrane present.

It has also been disputed as to whether they should be considered as true cells or not. If by definition a cell must contain a nucleus, they can hardly be considered as such, since the characters of the highly refractive bodies contained in them have nothing in common with nuclei. We shall give later on reasons for thinking that they may however, as a result of incubation, become veritable cells.

Another feature of the white yolk, according to His, is that in the region of the blastoderm it contains numerous large vacuoles filled with fluid; they are sufficiently large to be seen with the naked eye, but do not seem to be present in the ripe ovarian ovum.

9. It is now necessary to return to the blastoderm. In this, as we have already said, the naked eye can distinguish an opaque white rim surrounding a more transparent central area, in the middle of which again is a white spot of variable appearance. In an unfecundated cicatricula the white disc is simply marked with a number of irregular clear spaces, there being no proper division into a transparent centre and an opaque rim.

The opaque rim is the commencement of what we shall henceforward speak of as the area opaca; the central transparent portion is in the same way the beginning of the area pellucida. At this stage the distinction between these two areas depends entirely on the disposition of the white yolk beneath them, for the blastoderm when lifted up from the white yolk on which it rests appears uniform throughout. In the part corresponding to the area opaca the blastoderm rests immediately on the white yolk, which here forms a 
somewhat raised ring, often spoken of as the germinal wall; underneath the area pellucida is a shallow space containing a nearly clear fluid, to the presence of which the central transparency seems to be due. The white spot in the middle of the area pellucida appears to be the nucleus of Pander shining through.

Vertical sections of the blastoderm shew that it is formed of two layers. The upper of these two layers is composed, see Fig. 3, ep, of a single layer of cells, with their long axes arranged vertically, adhering together so as to form a distinct membrane, the edge of which rests upon the white yolk. After staining with silver nitrate, this membrane viewed from above shews a mosaic of uniform polygonal cells.

Each cell is composed of granular protoplasm filled with highly refractive globules; in most of the cells an oval nucleus may be distinguished, and is most probably present in all. They are of a uniform size (about $9 \mu$ ) over the opaque and the pellucid areas.

The under layer (Fig. 3, $l$ ), is composed of cells which vary considerably in diameter; but even the smaller cells of this layer are larger than the cells of the upper laver.

They are spherical, and so filled with granules and highly refractive globules, that a nucleus can rarely be seen in them: in the larger cells these globules contain a highly refractive body very similar to that present in the white yolk spheres, from the smaller kinds of which indeed they are scarcely distinguishable.

The cells of this layer do not form a distinct membrane like the cells of the upper layer, but lie as a somewhat irregular network of cells between the upper layer and the bed of white yolk on which the blastoderm rests. The lowest are generally the largest; in addition we find a few still larger cells generally separated by a small interval from the remainder of the cells of the lower layer, and resting directly upon the white yolk (Fig. 3, $b$ ). These are frequently spoken of as formative cells; they are however similar in character and indeed connected by gradations with the larger cells of the lower layer. Their mode of formation during segmentation will be subsequently described. 
FIG. 3.

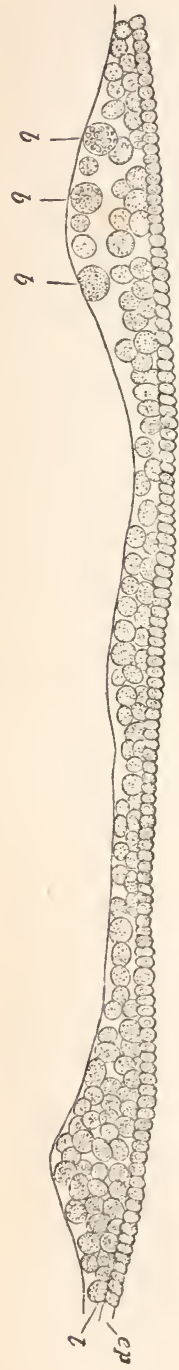

Section of a Blastodery of a Fowl's Egg at the COMMENCEMENT OF INCUBATION.

The thin but complete upper layer ep composed of columnar cells rests on the incomplete lower layer $l$, composed of larger and more granular bodies. The lower layer is thicker in some places than in others, and is especially thick at the periphery. The line below the under layer marks the upper surface of the white yolk. The larger so-called formative cells are seen at $b$, lying on the white yolk. The figure does not take in quite the whole breadth of the blastoderm; but the reader must understand that both to the right hand and to the left $e p$ is continued farther than $l$, so that at the extreme edge it rests directly on the white jolk.

Over nearly the whole of the blastoderm the upper layer rests on the under layer. At the circumference however the upper layer stretches for a short distance beyond the under layer, and here consequently rests directly on the white yolk, and forms that part of the blastoderm known as the area opaca.

10. To recapitulate:-In the normal unincubated hen's egg we recognize the blastoderm, consisting of a complete upper layer of smaller nucleated granular cells and a more or less incomplete under layer of larger cells, filled with larger granules; in these lower cells nuclei are rarely visible. 'The thin flat disc so formed rests, at the uppermost part of the entire yolk, on a bed of white yolk so disposed as to give rise to the appearance in the blastodermic disc itself of an area opaca and an area pellucida. The great mass of the entire yolk consists of the so-called yellow yolk composed of granular spheres. The white yolk is composed of smaller spheres of peculiar structure, and exists, in small part, as a thin coating around, and as thin concentric laminæ in the substance of the yellow yolk, but chiefly in the form of a flask-shaped mass in the interior of the yolk, the upper somewhat expanded top of the neck 
of which forms the bed on which the blastoderm rests. The whole yolk is invested with the vitelline membrane, this again with the white; and the whole is covered with two shell-membranes and a shell.

11. Such an egg has however undergone most important changes while still within the body of the hen; and in order to understand the nature of the structures which have just been described, it will be necessary to trace briefly the history of the egg from the stage when it exists as a so-called ripe ovarian ovum in the ovary of a hen up to the time when it is laid.

If one of the largest capsules of the ovary of a hen which is laying regularly be opened, it will be found to contain a nearly spherical (or more correctly, ellipsoidal with but slightly unequal axes) yellow body enclosed in a delicate membrane. 'I'his is the ovarian ovum or egg. Examined with care the ovum, which is tolerably uniform in appearance, will be found to be marked at one spot (generally facing the stalk of the capsule and forming the pole of the shorter axis of the ovum) by a small disc differing in appearance from the rest of the ovum. This dise is known as the germinal disc or discus proligerus. It consists of a lenticular mass of

Fig. 4.

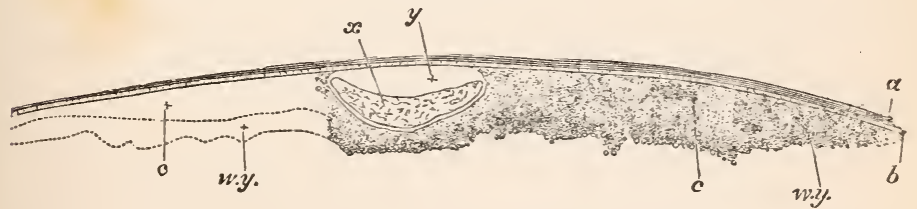

Section throvgh the Germisal Disc of the ripe Otarian Otda of a Fowl WHILE YET ENCLOSED IN ITS CAPSULE.

a. Connective-tissue capsule of the ovum. $\quad b$. epithelium of the capsule, at the surface of which nearest the ovum lies the vitelline membrane. $c$. granular material of the germinal disc, which becomes converted into the blastoderm. (This is not very well represented in the woodcut. In sections which hare been hardened in chromic acid it consists of fine granules.) w. y, white yolk, which passes insensibly into the fine granular material of the disc. $x$, germinal vesicle enclosed in a distinct nembrane, but shrivelled up by the action of the chromic acid. The material enclosed in the membrane of the vesicle is in the hardened specimens finely granular. $y$, space originally completely filled up by the germinal vesicle, before the latter was shrivelled up by the action of the chromic acid. 
protoplasm (Fig. 4, c), imbedded in which is a highly refractive globular or ellipsoidal body (Fig. 4, $x$ ), about $310 \mu$ in diameter, called the germinal vesicle, in the interior of which again is a small body, the germinal spot.

The rest of the ovum is known as the yolk. This consists of two elements, the white yolk- and the yellow yolk-spheres, which are distributed respectively very much in the same way as in the laid egg, the yellow yolk forming the mass of the ovum, and the white yolk being gathered underneath and around the disc (Fig. 4,w. y), and also forming a flaskshaped mass in the interior of the ovum. The delicate membrane surrounding the whole is the vitelline membrane.

Oellacher's (L'ntersuchung über die Furchung und Blatterbildung in Hüh. nereie. Studien aus dem Institute für experimentale pathologie in Wien aus dem Jahre 1869 , pt. I) account of the ovalian ovun differs considerably from that given above. He finds in the neighbourhood of the blastoderm a finely granular material, within which lies a body appearing circular when viewed from above, but having in section a somewhat quadrilateral shape; its side-walls, however, are curved, with their convexity turned inwards. At the bottom of it lies an oval cavity with doubly contoured walls, and at its upper surface placed somewhat excentrically a senicircular space filled with clear material.

Oellacher believes that the quadrilateral body which he thus describes is the germinal vesicle which has commenced to undergo a retrogressive metamorphosis. For the further stages in the metamorphosis, and for further par. ticulars, vide Section I3. Thye circular hole beneath the vesicle is probably merely filled with fluid and is due to the contractions of the germ.

12. When the ovarian ovum is ripe and about to be discharged from the ovary, its capsule is clasper by the dilated termination of the oviduct. 'The capsule then bursts, and the ovum escapes into the oviduct, its longer axis corresponding with the long axis of the oviduct, the germinal disc therefore being to one side. At the time of the bursting of the capsule the germinal vesicle disappears.

In describing the changes which take place in the oviduct, it will be convenient, following the order previously adopted, to treat first of all of the formation of the accessory parts of the egg. These are secreted by the glandular walls of the oviduct. This organ therefore requires some description. It may be sand to consist of four parts;-1st. The dilated proximal extremity. 2nd. A long tubular portion, opening by a narrow neck or isthmus into the 3rd portion, which is much dilated, and has been called the uterus; the 4th part is somewhat narrow, and leads from the uterus 
into the cloaca. The whole of the mucous membrane lining: the oviduct is largely ciliated.

The accessory parts of the egg are entirely formed in the 2nd and 3rd portions. The layer of albumen which immediately surrounds the yolk is first deposited; the chalazie are next formed. Their spiral character and the less distinctly marked spiral arrangement of the whole albumen is brought about by the motion of the egg along the spiral ridges into which the interior of the second or tubular portion of the oviduct is thrown. The spirals of the two chalazæ are in different directions. This is probably produced by their peripheral ends remaining fixed while the yolk to which their central ends are attached is caused to rotate by the contractions of the oviduct. During the formation of the chalazæ the rest of the albumen is also deposited; and finally the shell-membrane is formed in the narrow neck of the 2 nd portion, by the fibrillation of the most external layer of albumen. The egg passes through the 2nd portion in little more than 3 hours. In the 3rd portion the shell is formed. The mucous membrane of this part is raised into numerous flattened folds, like large villi, containing follicular glands. From these a thick white fluid is poured out, which soon forms a kind of covering to the egg, in which the inorganic particles are deposited. In this portion of the oviduct the egg remains from 12 to 18 hours, during which time the shell acquires its normal consistency. At the time of laying it is expelled from the uterus by violent muscular contractions, and passes with its narrow end downwards along the remainder of the oviduct, to reach the exterior.

13. We have now to trace out the changes which take place in the germinal disc, during the passage of the egg down the oviduct.

By the time when the egg becomes clasped by the expanded extremity of the oviduct the germinal vesicle has, according to Oellacier (loc cit. and also Archiv. fïr Micr. Anat. Vol. VIII. 18;2. p. I8), undergone still further retrouressive changes. It has now become very much flattened and closely applied to the vitelline membrane. Both this and former stages, if we may judge from the analogy of osseous fishes, are preparatory to the whole germinal vesicle being bodily ejected from the germinal disc. For further particulars vide Oellacher, A reniv. fïr Micr. Anat. Vol. virr. pp. I-26.

Impregnation occurs in the upper portion of the oviduct; the spermatozoa being found actively moving in a fluid which is there contained. 
It is not certain whether impregnation takes place previous to the deposition of the albumen, or whetier the spermatozoa bore their way through the albumen. The former would appear to be the more probable view, though the fact that Oellacher has found spermatozoa in the albumen, speaks in favour of their being involved in the depositing albumen, and so being brought in contact with tue blastoderm.

According to Coste, Histoire du développement des corps organizés, the access of the cock to the hen once in seven days is sufficient.

We have no positive evidence that the spermatozoa make their way through the vitelline membrane and so gain access to the germinal disc; but, as will be seen in a later part of this work, analogy renders such an event probable.

14. At about the time when the shell is being formed round the egg, the germinal disc undergoes a remarkable change, known as segmentation. We shall have occasion to treat more fully of the nature of segmentation when we come to consider the amphibian ovum in which the various steps of the process may be more easily and satisfactorily traced. Meanwhile, inasmuch as the segmentation of the

FIG, 5 .

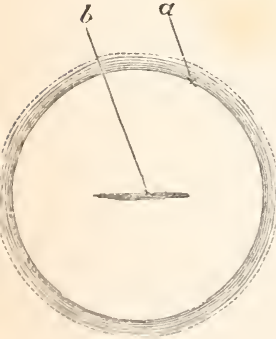

A

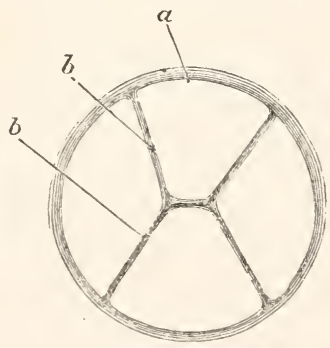

B

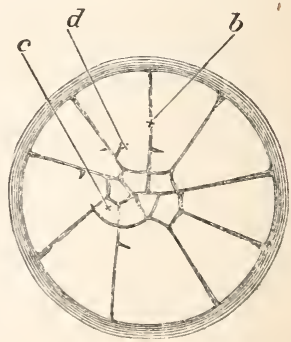

C

\section{Surface Views of the earty Stages of the Segmentation in a Fowl's Egg. (After Coste.)}

$A$ represents the earliest stage. The first furrow $(b)$ has begun to make its appearance in the centre of the germinal disc, whose periphery is marked by the line $a$. In $B$, the first furrow is completed right across the disc, and a second similar furrow at nearly right angles to the first has appeared. The disc thus becomes divided somewhat irregularly into quadrants by four (half) furrows. In a later stage $(C)$ the mericlian furrows $b$ have increased in number, from four, as in $B$, to nine, and cross furrows have also made their appearance. The disc is thus cut up into small central $(c)$ and larger peripheral $(d)$ segments. Several new cross furrows are seen just beginning, as $e x . g r$. close to the end of the liwe of reference $d$. 
germinal disc of a hen's egg differs materially from the segmentation of the entire ovum of an amphibian, the former may briefly be described here.

Viewed from above, a furrow is seen to make its appearance, running across the germinal disc and dividing it into two halves (Fig. 5, A). This primary furrow is succeeded by a second at right angles to itself. The surface thus becomes divided into four segments or quadrants (Fig. 5, $B$ ). Each of these is again bisected by radiating furrows, and thus the number of segments is increased from four to eight (it may be seven or nine). The central portion of each segment is then, by a cross furrow, cut off from the peripheral portion, giving rise to the appearance of a number of central smaller segments, surrounded by more external elongated segments (Fig. $5, C$ ).

Division of the segments now proceeds rapidly by means of furrows running apparently in all directions. And it is

Fig. 6.

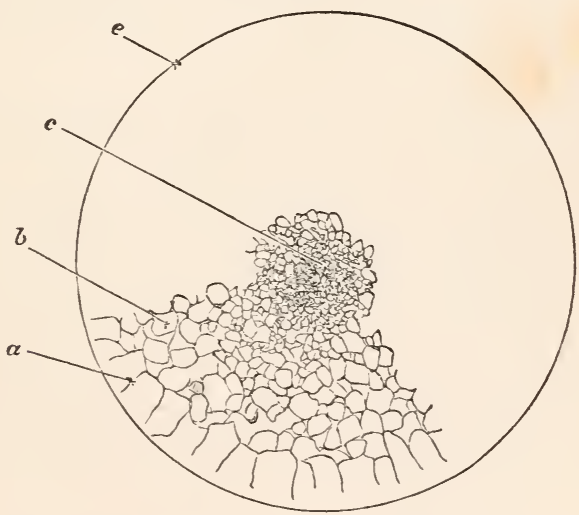

Surface View of the Germinal Disc of a Hen's Egg during the later Stages of Segalentation. (Chromic Acid Preparation.)

At $c$ in the centre of the disc the segmentation masses are very small and numerous. At $b$. nearer the edge, they are larger and fewer; while those at the extreme margin $a$ are largest and fewest of all. It will be noticed that the radiating furrows marking off the segments $a$ do not as yet reach to the extreme margin $e$ of the disc.

The drawing is completed in one quadrant only; it will of course be understood that the whole circle ought to be filled up in a precisely similar manner. 
important to note that the central segments divide more rapidly than the peripheral, and consequently become at once smaller and more numerous (Fig. 6).

Meanwhile sections of the hardened blastoderm teach us that segmentation is not confined to the surface, but extends through the mass of the blastoderm; they shew us moreover that division takes place by means of not only vertical, but also horizontal furrows, i.e. furrows parallel to the surface of the disc (Fig. T).

\section{FIG. 7 .}

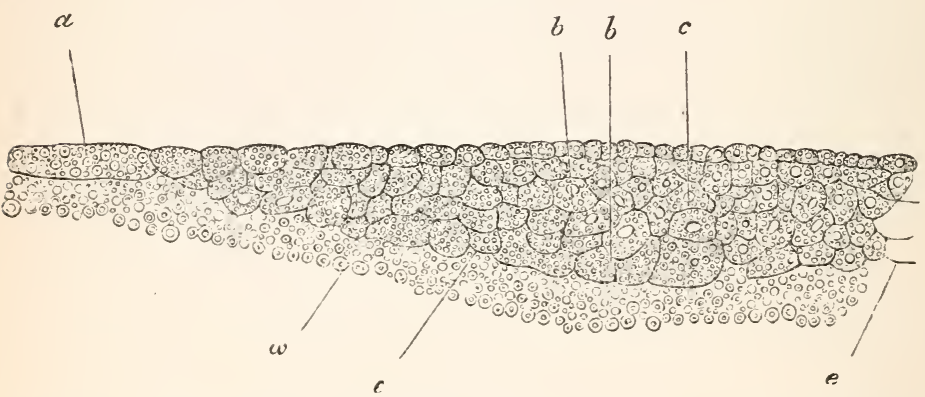

\section{Section of the Germinal Disc of a Fowl during the Later Stages} of Seguentation.

The section, which represents rather more than half the breadth of the blastoderm (the midale line being shewn at $c$ ), shews that the upper and central parts of the disc segment faster than those below and towards the periphery. At the periphery the segments are still very large. One of the larger segments is shewn at $a$. In the majority of segments a nucleus can be seen; and it seems probable that a nucleus is present in them all. Most of the segments are filled with highly refracting spherules, but these are more numerous in some cells (especially the larger cells near the yolk) than in others. In the central part of the blastoderm the upper cells have commenced to form a distinct layer. No segmentation-cavity is present.

$a$. large peripheral cell. $b$. larger cells of the lower parts of the blastoderm. $c$. middle line of blastoderm. $e$. edge of the blastoderm adjoining the white yolk. w. white yolk.

In this way, by repeated division, or segmentation, the original germinal disc is cut up into a large number of small rounded masses of protoplasm, which are smallest in the centre, and increase in size towards the periphery. The segments lying uppermost are moreover smaller than those beneath, and thus the establishment of the two layers of the blastoderm is foreshadowed. 
According to Oellacher, Studien aus dem Ins. f. Exper. Pathol. Vien. i S69, p. I, sections taken through the centre of the germinal disc at the byinning of segmentation shew a somewhat uneven vertical furrow, ending below in a small triangular space, where it joins a nearly horizontal furrow which meets the surface of the erg at some little distance on either side of the vertical furrow. It seems certain that these fist-formed furrows do not iuclude the whole of the germinal disc, whose limits at this stage are however uncertain. In the later stages of segmentation not only do the first-former segments become further divided, but segmentation also extends into the remainder of the germinal disc. Goette, Archiv. Micr. Anat. А. $1+5$, indeed maintrins that segmentation (at a later period) even involves material which is uudoubterly white yolk. He describes nuclei as making their appearance in the upper surfare of the ber of white yolk, and the substance round them as rising up in the form of papilice, which are subsequently constricted off and set free as supplementary segmentation masses. It is these, acco ding to him, which give ris to the formative cells spoken of in the next paragraph. He states that they continue to be formed long after the commencement of incubation. We shail returu to this subject, when we come to discuss more fully the nature of the process of segmentation, in describing the ova of other classes of vertebrates.

Between the segmented germinal disc, which we may now call the blastoderm, and the bed of white yolk on which it rests, a space containing fluid makes its appearance. This, gradually increasing in all dimensions, may be called the segmentation-cavity.

15. As development proceeds, segmentation reaches its limits in the centre, but continues at the periphery, and thus eventually the masses at the periphery become of the same size as those in the centre.

The distinction however between an upper and a lower layer becomes more and nore obvious.

The masses of the upper layer arrange themselves, side by side, with their long axes vertical; their nuclei become very distinct. In fact they form a membrane of columnar nucleated cells.

The masses of the lower layer, remaining larger than those of the upper layer, continue markedly granular and round, and form rather a close irregular network than a distinct membrane. In them nuclei are either wholly absent or at least not readily visible.

It seems more probable that the nucleus is hidden than that it is really absent. In the earliest stages of segmentation which we have examined when the segments were still few in number, a very large proportion of both great and snall segments contained large well-formed nuciei. These nucl-ated segments, which were found in hoth the superficial and deeper portions of the lisc, were invariably those in which the granules were fur some reason or other few and fine; in fact, wherever the granules were not sufficientls numerous to render tise body of the segment too opayue, there a nucleus could be detected. 
We were thus led to the conclusion that a nucleus really existed in all. It is of course quite possible that the clearer nucleated masses eventually come to the surface and leave the more granular and opaque masses to form the lower layer; but it is much more likely that they do not, and that the granular condition of the cells of the lower layer of the fully formed blastoderm is on the one hand the result of their being in immediate contact with the excessively granular white yolk-cells, and on the other the cause of their nuclei not being seen. We have a somewhat analogous case in the invisibility of the nucleus in the early stages of the amphibian blood-corpuscle.

At the time when the segmentation-spheres in the centre are smaller than those at the periphery, and those above are also smaller than those below, a few large spherical rrasses begin to separate from the remainder (or to arise by a continued process of segmentation from the bed of white yolk), and to rest directly on the white yolk, at the bottom of the shallow segmentation-cavity. They contain either numerous small nucleated spherules, or fine granules; the spherules precisely resembling the smaller spheres of white yolk. These loose spherical masses are the formative cells already spoken of.

Thus the original germinal disc of the ovarian ovum, its germinal vesicle having disappeared, becomes, by the process of segmentation, converted into a blastoderm such as is met with in the egg when laid, into an upper layer of columnar nucleated cells, and into a lower layer of irregularly disposed rounded masses which have not yet definitely acquired the character of cells, accompanied by a few stray "formative" cells lying loose in the segmentation-cavity. 


\section{CHAPTER II.}

\section{A BRIEF SUMIIARY OF THE WHOLE HISTORY OF INCUBATION.}

1. Step by step the simple two-layered blastoderm described in the previous chapter is converted into the complex organism of the chick. The details of the many changes through which this end is reached will perhaps be rendered more intelligible if we prefix to the special history of them a brief summary of the general course of events from the beginning to the end of incubation.

In the first place, it is to be borne in mind that the embryo itself is formed in the area pellucida, and in the area pellucida alone. The area opaca in no part enters directly into the body of the chick; the structures to which it gives rise are to be regarded as appendages, which sooner or later disappear.

2. The blastoderm at starting consists of two layers. Very soon a third layer makes its appearance between the other two. These three layers, the establishment of which is a fact of fundamental importance in the history of the embryo, are called respectively the upper, middle and lower layers, or epiblast, mesoblast and hypoblast.

This triple division corresponds roughly, though not exactly, to the old division into serous, vascular and mucous layers.

3. The blastoderm which at first, as we have seen, lies like a watch-glass over the segmentation-cavity, its margin resting on the circular germinal wall of white yolk, spreads, as a thin circular sheet, over the yolk, immediately under the vitelline membrane. Increasing uniformly at all points of its circumference, the blastodermic expansion covers more and more of the yolk, and at last, reaching its opposite pole 
completely envelopes it. Thus the whole yolk, instead of being enclosed as formerly by the vitelline membrane alone, comes to be also enclosed in a bag formed by the blastoderm.

It is not however until quite a late period that the complete closing in at the opposite pole takes place, so that the extension of the blastoderm must be thought of as going on during nearly the whole period of incubation.

Both the area opaca and the area pellucida share in this enlargement, but the area opaca increases much more rapidly than the area pellucida, and plays the principal part in encompassing the yolk.

4. The mesoblast, in that part of the area opaca which is nearest to the area pellucida, becomes the seat of peculiar changes, which result in the formation of blood-vessels. Hence this part of the area opaca is called the vascular area.

5. The embryo itself may be said to be formed by a folding off of the central portion of the area pellucida from the rest of the blastoderm. At first the area pellucida is quite flat, or, inasmuch as it forms part of the circumference of the yolk, slightly but uniformly curved. Very soon, however, there appears at a certain spot a semilunar groove, at first small, but gradually increasing in depth and extent; this groove. which is represented in section in the diagram (Fig. $8, A)$, breaks the uniformity of the level of the area pellucida. It may be spoken of as a tucking in of a small portion of the blastoderm in the form of a crescent. When viewed from above, it presents itself as a curved line (the hinder of the two concentric curved lines in front of $A$ in Fig 11), which marks the hind margin of the groove, the depression itself being hidden. In a vertical longitudinal section carried through the middle line, we may recognize the following parts (Fig. 8, A, or on a larger scale Fig. 9, which also shews details which need not be considered now). Beginning at what will become the posterior extremity of the embryo, (the left-hand side of the figure in each case), and following the surface of the blastoderm forwards (to the right in the figures), the level is maintained for some distance, and then there is a sudden descent, the blastoderm bending round and pursuing a precisely opposite direction to its previous one, running backwards instead of forwards, for some distance. It soon however turns round again, and once more running 
FIG. 8.
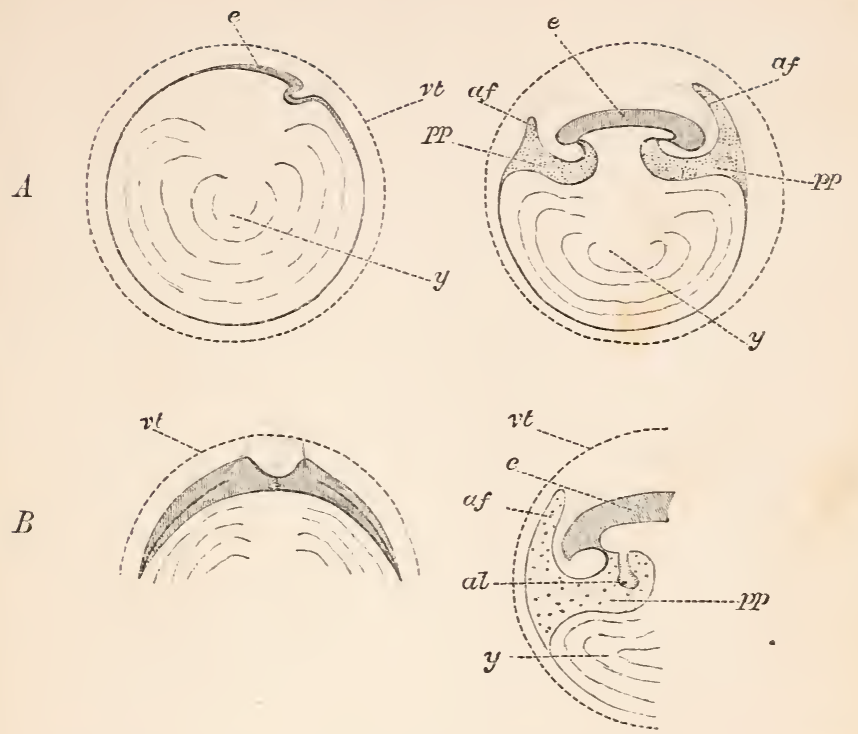

Fig. $8, A$ to $N$ forms a series of purely diagrammatic representations introduced to facilitate the comprehension of the manner in which the body of the embryo is formed, and of the various relations of the yolk-sac, amnion and allantois.

In all $v t$ is the vitelline membrane, placed, for convenience sake, at some distance from its contents, and represented as persisting in the later stages: in the actual egg it is in direct contact with the blastoderm (or yolk), and early ceases to have a separate existence. In all $e$ indicates the embryo, $p p$ the general pleuro-peritoneal space, $a f$ the folds of the amuion, $a$ the amnion proper, ae or ac the cavity holding the liquor amnii, al the allantois, $a^{\prime}$ the alimentary canal, $y$ or $y s$ the yolk or yolk-sac.

$A$, which may be considered as a vertical section taken longitudinally along the axis of the embryo, represents the relations of the parts of the egg at the time of the first appearance of the head-fold, seen on the right-hand side of the blastoderm $e$. The blastoderm is spreading both behind (to the left hand in the figure), and in front (to right hand) of the head-fold, its limits being indicated by the sharing and thickening for a certain distance of the margin of the yolk $y$. As yet there is no fold on the left side of $e$ corresponding to the head-fold on the right, while therefore the front limit of the embryo, as distinguished from the blastoderm, is marked out by the head-fold, there is at present no tail-fold, and therefore no hind limit.

$B$ is a rertical transverse s ction of the same period drawn for convenience sake on a larger scale (it should have been made flatter and less curved). It shews that the blastoderm (vertically shaded) is extending laterally as well as fore and aft, in fact in all directions; but there are no lateral folds, and there- 
fore no lateral limits to the body of the embryo as distinguished from the blastcderm.

Incidentally it shews the formation of the medullary groove by the rising up of the laminæ dorsales. Beneath the section of the groove is seen the rudiment of the notochord. On either side a line indicates the cleavage of the mesoblast just commencing. This cleavage, it will be seen, does not exist in the more central parts of the embryo.

In $C$, which represents a vertical longitudinal section of later date, both head-fold (on the right) and tail-fold (on the left) have advanced considerably. The alimentary canal is therefore closed in both, in front and behind, but is in middle still widely open to the yolk $y$ below. Though the axial parts of the embryo have become thickened by growth, the body-walls are still thin; in them however is seen the cleavage of the mesoblast, and the divergence of the somatopleure and splanchnopleure. The splanchnopleure both at the head and at the tail is folded in to a greater extent than the somatopleure, and
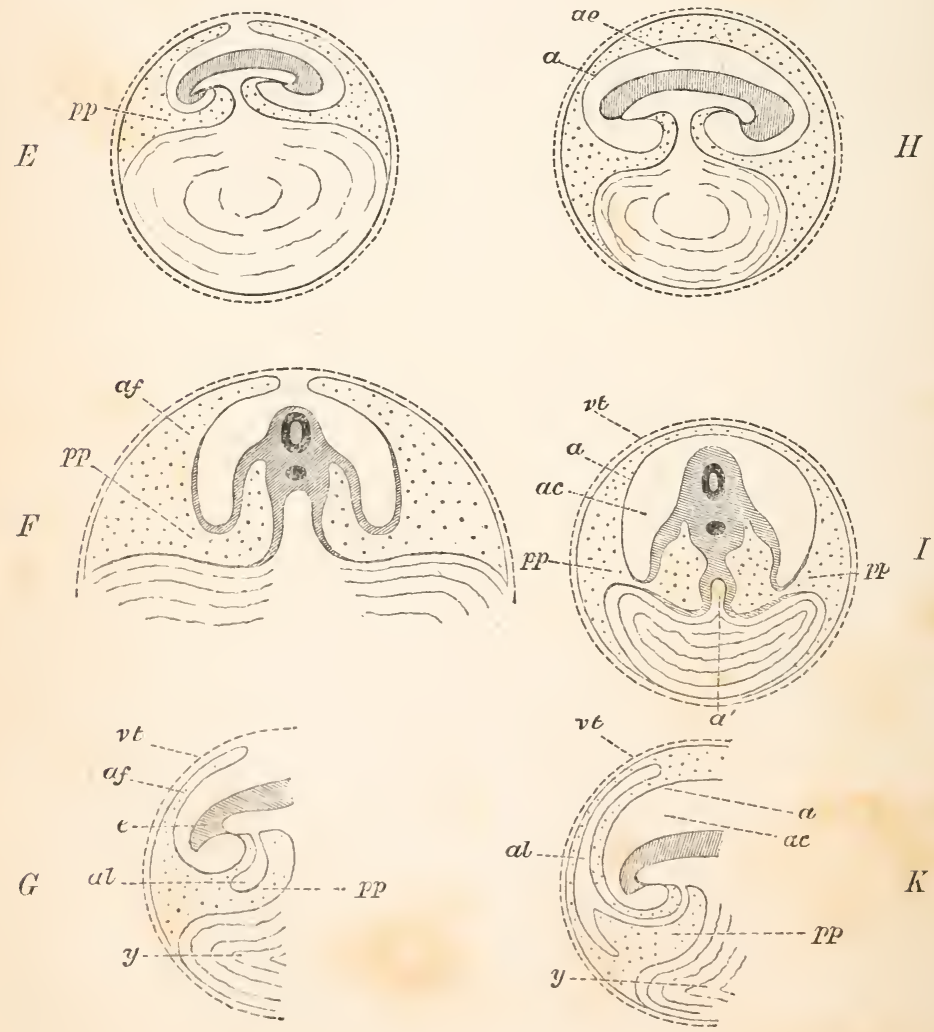
forms the still wide splanchnic stalk. At the end of the stalk, which is as yet short, it bends outwards again and spreads over the top of the yolk. The somatopleure folded in less than the splanchnopleure to form the wider somatic stalk, sooner bends round and runs outwards again. At a little distance from both the head and the tail it is raised up into a fold, af, af, that in front of the head being the highest. These are the amniotic fo:ds. Descending from either fold, it speedily joins the splanchnopleure again, and the two, once more united into an uncleft membrane, extend some way downwards over the yolk, the limit or outer margin of the opaque area not being shewn. All the space between the somatopleure and the splanchnopleure is shaded with dots, $p p$. Close to the body this space may be called the pleuroperitoneal cavity; but outside the body it runs up into either amniotic fold, and also extends some little way over the yolk.

$D$ represents the tail-end at about the same stage on a more enlarged scale, in order to illustrate the position of the allantois al (which was for the sake of simplicity omitted in (C), shewn as a bud from the splanchnopleure, stretching downwards into the pleuroperitoneal cavity $p p$. The dotted area representing as before the whole space between the splanchnopleure and the somatopleure, it is evident that a way is open for the allantois to extend from its present position into the space between the two walls of the amniotic fold $a f$.

$E$, also a longitudinal section, represents a stage still farther advanced. Both splanchnic and somatic stalks are much narrowed, especially the former, the cavity of the alimentary canal being now connecterl with the cavity of the yolk by a mere canal. 'Tise folds of the amnion are spreading over the top of the embryo and nearly meet. Each fold cousists of two walls or limbs, the space between which (dotted) is as before merely a part of the space between the somatopleure and splanchoopleure. Between these arched amniotic folds and the body of the embryo is a space not as yet entirely closed in.

$F$ represents on a different scale a transverse section of $E$ taken through the middle of the splanchnic stalk. The black ring in the body of the embryo shews the position of the neural canal, below which is a black spot, marking the notochord. On either side of the notochord the divergence of somatopleure and splanchnopleure is obvious. The splanchnopleure, more or less thickened, is somewbat bent in towards the middle line, but the two sides do not unite, the alimentary canal being as yet open below at this sput; after converging somewhat they diverge again and run outwards over the yolk. The somatopleure folded in to some extent at first to form the body-walls (which are here made too thick), soc $\mathrm{n}$ benıls outwards again, and almost immediately is raised up into the lateral folds of the amnion af. The continuity of the pleuroperitoneal cavity within the body with the interior of the amniotic fold outside the body is evident; both cavities are dotted. It will of course be understood that this is a purely diagrammatic representation, the various cavities, \&c., being exaggerated in order to shew their relations more clearly.

$G$, which corresponds to $D$ at a later stage, is introduced to shew the manner in which the allantois, now a distinctly hollow body, whose cavity is continuous with that of the alimentary canal, becomes directed towards the amniotic fold.

In $H$ a longitudinal, and $I$ a transverse section of later date, great changes have taken place. The several folds of the amnion have met and coalesced above the body of the embryo. The inner limbs of the several folds have united into a single membrane $(a)$, which encloses a space $(a e)$ round the embryo. This membrane $a$ is the amnion proper, and the cavity within it, i.e. between it and the embryo, is the cavity of the amnion containing the liquor amnii. 
It will be seen that the amnion $a$ now forms in every direction the termination of the somatopleure; the peripheral portions of the somatopleure, the united outer or descending limbs or walls of the folds of in $C, D, F, G$ having been cut adrift, and now forming an independent continuous membrane, the chorion, immediately underneath the vitelline membrane.

In $I$ the splanchnopleure is seen converging to complete the closure of the alimentary canal $a^{\prime}$, even at the stalk (elsewhere the canal has of course long been closed in), and then spreading outwards as usual over the yolk. The point at which it unites with the somatopleure, narking the extreme limit of the cleavage of the mesoblast, is now much nearer the lower pole of the diminished yolk.

As a result of these several changes, a great increase in the dotterl space has taken place. It is now possible to pass from the actual peritoneal cavity within the body, on the one hand round a great portion of the circumference of the yolk, and on the other hand above the amnion $a$, in the space between it and the chorion.

Into this space the allantois is seen spreading in $K$ at $a l$.
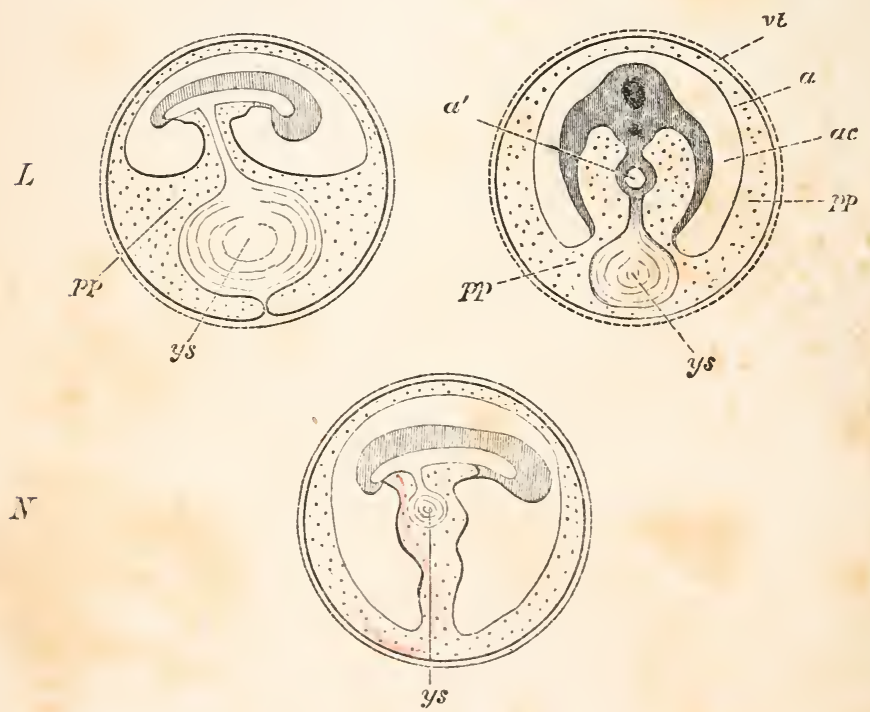

In $L$ the splanchnopleure has completely invested the yolk-sac, but at the lower pole of the yolk is still continuous with that peripheral remnant of the somatopleure now called the chorion. In other words, cleavage of the mesoblast has been carried all round the yolk $(y s)$ except at the very lower pole.

In $M$ the cleavage has been carried through the pole itself; the peripheral portion of the splanchnopleure forms a complete investnent of the yolk quite uncomnected with the peripheral portion of the somatopleure, which now exists as a continuous membrane lining the interior of the shell. The yolk sac $(y s)$ is therefore quite loose in the pleuroperitoneal cavity, being connected only with the alimentary canal $\left(a^{\prime}\right)$ by a solid pedicle. 
Lastly, in $N$ the yolk sac $(y s)$ is shewn being withdrawn into the cavity of the body of the embryo. The allantois is as before, for the sake of simplicity, omitted; its pedicle would of course lie by the side of $y s$ in the somatic stalk inarked by the usual dotted shading.

It may be repeated that the above are diagrams, the various spaces being shewn distended, whereas in many of them in the actual egg the walls have collapsed, and are in near juxtaposition.

FiG. 9.

$N C$ :

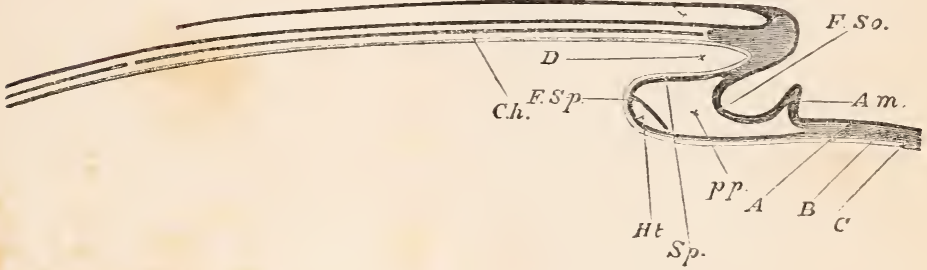

Diagramatio Longitudixal Section throdgh the Axis of an Embryo.

The section is supposed to be made at a time when the head-fold has commenced but the tail-fold has not yet appeared.

$F$. So. fold of the somatopleure. F. Sip. fold of the splanchnopleure.

The line of reference $F$. So. is placed in the lower bay, outside the embryo. The line of $D$ is placed in the upper bay inside the embryo; this will remain as the alimentary canal. Both folds $(F$. So., $F . S p$. $)$ are parts of the head-fold, and are to be thought of as continually travelling onwards (to the left) as development proceeds.

$p p$. space between somatopleure and splanchnopleure: pleuroperitoneal cavity. $A \mathrm{~m}$. commencing (head) fold of the amnion.

A fuller explanation is given under Fig. 16.

forward, with a gentle ascent, regains the original level. As seen in section, then, the blastoderm at this spot may be said to be folded up in the form of the letter 2 . This fold we shall alvays speak of as the head-fold. In it we may recognize two limbs: an upper limb in which the curve is directed forwards, and its bay, opening backwards, is underneath the blastoderm, i.e. as we shall see, inside the embryo (Fig. 9, $D$ ); and an under limb in which the curve is directed backwards, and its bay, opening forwards, is above the blastoderm, i.e. outside the embryo. If an $q$ like the above, made of some elastic material, were stretched laterally, the effect would be to make both limbs longer and proportionally narrower, and 
their bays, instead of being shallow cups, would become more tubular. Such a result is in part arrived at by the growth of the blastoderm; the upper limb of the $p_{1}$ is continually growing forward (but, unlike the stretched elastic model, increases in all its dimensions at the same time), and the lower limb is as continually lengthening backwards; and thus both upper and lower bays become longer and longer. This we shall hereafter speak of as the travelling backwards of the head-fold.

The two bays do not however both become tubular. The section we have been speaking of is supposed to be taken vertically along a line, which will afterwards become the axis of the embryo; and the lower bay of the $Z$ is a section of the crescentic groove mentioned above, in its middle or deepest part. On either side of the middle line the groove gradually becomes shallower. Hence in sections taken on either side of the middle line or axis of the embryo (above or below the plane of the figures), the groove would appear the less marked the farther the section from the middle line, and at a certain distance would disappear altogether. It must be remembered that the groove is at first crescent-shaped, with its corcavity turned towards what will be the hind end of the embryo (Fig. 11). As the whole head-fold is carried farther and farther back, the horns of the crescent are more and more drawn in towards the middle line, the groove becoming first semicircular, then horse-shoe-shaped. In other words, the headfold, instead of being a simple fold running straight backwards, becomes a curved fold with a central portion in front running backwards, and two side portions rumning in towards the middle line. The effect of this is that the upper bay of the $e_{1}$ (that within the embryo) gets closed in at the sides as well as in the front, and thus speedily becomes tubular. The under bay of the 2 (that outside the embryo) remains of course open at the sides as in front, and forms a sort of horse-shoe-shaped ditch surrounding the front end of the embryo.

We have dwelt thus at length on the formation of the head-fold, because, unless its character's are fairly grasped, much difficulty may be found in understanding many events in the history of the chick. The reader will perhaps find the matter easier to comprehend if he makes for himself a rough 
model, which he easily can do by spreading a cloth out flat to represent the blastoderm, placing one hand underneath it, to mark the axis of the embryo, and then tucking in the cloth from above under the tips of his fingers. The fingers, covered with the cloth and slightly projecting from the level of the rest of the cloth, will represent the head, in front of which will be the semicircular or horse-shoe-shaped groove of the head-fold.

At its first appearance the whole 2 may be spoken of as the head-fold, but later on it will be found convenient to restrict the name chiefly to the lower limb of the 2 .

Some time after the appearance of the head-fold, an altogether similar but less conspicuous fold makes its appearance, at a point which will become the posterior end of the embryo. This fold, which travels forwards just as the head-fold travels backwards, is the tail-fold (Fig. 8, C).

In addition, between the head- and the tail-fold two lateral folds appear, one on either side. These are simpler in character than either head-fold or tail-fold, inasmuch as they are nearly straight folds directed inwards towards the axis of the body (Fig. 8, F), and not complicated by being crescentic in form. Otherwise they are exactly similar.

As these several folds become more and more developed, the head-fold travelling backwards, the tail-fold forwards, and the lateral folds inwards, they tend to unite in the middle point; and thus give rise more and more distinctly to the appearance of a small tubular sac seated upon, and connected, by a continually-narrowing hollow stalk, with that larger sac which is formed by the extension of the rest of the blastoderm over the whole yolk.

The smaller sac we may call the "embryonic sac," the larger one "the yolk-sac." As incubation proceeds the smaller sac (Fig. S), gets larger and larger at the expense of the yolksac (the contents of the latter being gradually assimilated by nutritive processes into the tissues forming the growing walls of the former, not directly transferred from one cavity into the other). Within a day or two of the hatching of the chick, at a time when the yolk-sac is still of some considerable size, or at least has not yet dwindled away altogether, and the development of the embryonic sac is nearly complete, the yolk-sac (Fig. 8, $N$ ) is slipped into the body of 
the embryo, so that ultimately the cmbryonic sac alone remains.

6. The embryo, then, is formed by a folding off of a portion of the blastoderm from the yolk-sac. The general outline of the embryo is due to the direction and shape of the several folds which share in its formation; these, while preserving a nearly perfect bilateral symmetry, present marked differences at the two ends of the embryo. Hence from the very first there is no difficulty in distinguishing the end which will be the head from that which will be the tail.

In addition to this, the tubular sac of the embryo, while everywhere gradually acquiring thicker and thicker walls, undergoes at various points, through local activities of growtli in the form of thickenings, ridges, buds or other processes, many modifications of the outline conferred upon it by the constituent folds. Thus bud-like processes start out from the trunk to form the rudiments of the limbs, and similar thickenings and ridges give rise to the jaws and other parts of the face. By the unequal development of these outgrowths the body of the chick is gradually moulded into its proper outward shape.

7. Were the changes which take place of this class only, the result would be a tubular sac of somewhat complicated outline, but still a simple tubular sac. Such a simple sac might perhaps be roughly taken to represent the body of many an invertebrate animal; but the typical structure of a bird or other vertebrate animal is widely different. It may very briefly be described as follows.

First there is, above, a canal running lengthways along the body, in which are lodged the brain and spinal cord. Below this neural tube is an axis represented by the bodies of the vertebra and their continuation forwards in the structures which form the base of the skull. Underneatl this, again, is another tube closed in above by the axis, and on the sides and below by the body-walls. Enclosed in this second tube, and suspended from the axis, is a third tube, consisting of the alimentary canal with its appendages (liver, salivary glands, lungs, \&c., which are fundamentally mere diverticula from one simple canal). The cavity of the outer tube, which also contains the heart and other parts of the vascular system, is the general 
body cavity; it is divided into a thoracic or pleural, and an abdominal or peritoneal cavity; these two cavities are, however, from their mode of origin, portions of one and the same tube. Thus a transverse section of a vertebrate animal always shews the same fundamental structure: above a single tube, below a double tube, the latter consisting of one tube enclosed within another, the inner being the alimentary canal, the outer the general cavity of the body. Into such a triple tube the simple tubular embryonic sac of the chick is converted by a series of changes of a remarkable character.

The upper or neural tube is formed in the following way. At a very early period the upper surface of the blastoderm in the region which will become the embryo, is raised up into two ridges or folds which run parallel to each other at a short distance on either side of what will be the long axis of the embryo, and thus leave between them a shallow longitudinal groove (Fig. 8, $B$, also Figs. 11, 12, m.c). As these ridges, which bear the name of medullary folds, increase in height they arch over towards each other and eventually meet and coalesce in the middle line, thus converting the groove into a canal, which at the same time becomes closed at either end (Fig. 8, F, I, also Fig. 13.-.M.). The cavity so formed is the cavity of the neural tube, and eventually becomes the cerebro-spinal canal.

The lower double tube, that of the alimentary canal, and of the general cavity of the body, is formed in an entirely different way. It is, broadly speaking, the result of the junction and coalescence of the fundamental embryonic folds, the head-fold, tail-fold, and lateral folds; in a certain sense the cavity of the body is the cavity of the tubular sac described in the last paragraph.

But it is obvious that a tubular sac formed by the folding in of a single sheet of tissue, such as we have hitherto considered the blastoderm to be, must be a simple tubular sac possessing a single cavity only. The blastoderm however does not long remain a single sheet, but speedily becomes a louble sheet of such a kind that, when folded in, it gives rise to a double tube.

Very early the blastoderm becomes thickened in the region of the embryo, the thickening being chiefly due to an increase in the middle layer or mesoblast, while at the same 
time it becomes split or cleft horizontally over the greater part of its extent into two leaves, an upper leaf and a lower leaf. In the neighbourhood of the axis of the body, beneath the neural tube, this cleavage is absent (Fig. $8, B$; also Figs. 13-20), in fact, it begins at some little distance on either side of the axis and spreads thence into the periphery in all directions. It is along the thickened mesoblast that the cleavage takes place, the upper part of the mesoblast uniting with epiblast to form the upper leaf, and the lower part with the hypoblast to form the lower leaf.

In the fundamental folds both leaves are involved, both leaves are folded downwards and inwards, both leaves tend to meet in the middle below; but the lower leaf is folded in more rapidly, and thus diverges from the upper leaf, a space being gradually developed between them (Fig. 8). In course of time the several folds of the lower leaf meet and unite to form an inner tube quite independently of the upper leaf, whose own folds in turn meet and unite to form an outer tube separated from the inner one by an intervening space. The inner tube is the alimentary canal which is subsequently perforated at both ends to form the mouth and anus; the walls of the outer tube are the walls of the body, and the space between the two tubes is the general "serous cavity," which being subsequently divided into pleural and peritoneal portions, may be spoken of as the pleuroperitoneal cavity.

Hence the upper (or outer) leaf of the blastoderm, from its giving rise to the body-walls, is called the somatopleure ${ }^{1}$; the lower (or inner) leaf, from its forming the alimentary canal and its tributary viscera, the splanchnopleure ${ }^{2}$.

This horizontal splitting of the blastoderm into a somatopleure and a splanchnopleure, which we shall hereafter speak of as the cleavage of the mesoblast, is not confined to the region of the embryo, but gradually extends over the whole of the yolk-sac. Hence in the later days of incubation the yolksac comes to have two distinct coats, an inner splanchnopleuric and an outer somatopleuric investment, separable from each other all over the sac. We have seen that, owing to the manner of its formation, the 'embryonic sac' is connected with the 'yolk-sac' by a continual narrowing hollow 
stalk; but this stalk must, like the embryonic sac itself, be a double stalk, and consist of a smaller inner stalk within a larger outer one, Fig. 8, E,H. The folds of the splanchnopleure, as they tend to meet and unite in the middle line below, give rise to a continually narrowing hollow stalk of their own, a splanchnic stalk, by means of which the walls of the alimentary canal are continuous with the splanchnopleuric investment of the yolk-sac, and the interior of that canal is continuous with the yolk inside the yolk-sac. In the same way the folds of the somatopleure form a similar stalk of their own, a somatic stalk, by means of which the bodywalls of the chick are continuous (for some time; the continuity, as we shall see, being eventually broken by the development of the amnion) with the somatopleuric investment of the yolk-sac; and the pleuroperitoneal cavity of the body of the chick is continuous with the narrow space between the two investments of the yolk-sac.

At a comparatively early period the canal of the splanchnic stalk becomes obliterated, so that the material of the yolk can no longer pass directly into the alimentary cavity, but has to find its way into the body of the chick by absorption through the blood-vessels. The somatic stalk, on the other hand, remains widely open for a much longer time; but the somatic shell of the yolk-sac never undergoes that thickening which takes place in the somatic walls of the embryo itself; on the contrary, it remains thin and insignificant. When accordingly in the last days of incubation the greatly diminished yolk-sac with its splanchnic investment is withdrawn into the rapidly enlarging abdominal cavity of the embryo, the walls of the abdomen close in and unite, without any regard to the shrivelled, emptied somatopleuric investment of the yolk-sac, which is cast off as no longer of any use. (Fig. 8. Compare the series.)

8. Very closely connected with the cleavage of the mesoblast and the division into somatopleure and splanchnopleure, is the formation of the amnion, all mention of which was, for the sake of simplicity, purposely omitted in the description just given.

The amnion takes its origin from certain folds of the somatopleure, and of the somatopleure only, in the following way. 
At a time when the cleavage of the mesolulast has somewhat advanced, there appears, a little way in front of the semilunar head-fold, a second fold (Fig. 11, also Fig 8, C.), running more or less parallel or rather concentric with the first and not unlike it in general appearance, though differing widely from it in nature. In the head-fold the whole thickness of the blastoderm is involved; in it both somatopleure and splanchnopleure (where they exist, i.e. where the mesoblast is cleft,) take part. This second fold, on the contrary, is limited entirely to the somatopleure. Compare Figs. 8 and 9. In front of the head-fold, and therefore altogether in front of the body of the embryo, the somatopleure is a very thin membrane, consisting only of epiblast and a very thin layer of mesoblast; and the fold we are speaking of is, in consequence, itselt thin and delicate. Rising up as a semilunar fold with its concavity directed towards the embryo (Fig. S, C, af.), as it increases in height it is gradually drawn backwards over the developing head of the embryo. The fold thus covering the head is in due time accompanied by similar folds of the somatopleure, starting at some little distance behind the tail, and at some little distance from the sides (Fig. 8, $C, D, E, F^{\prime}$ ). In this way the embryo becomes surrounded by a series of folds of thin somatopleure, which form a continuous wall all round it. All are drawn gradually over the body of the embryo, and at last meet and completely coalesce (Fig. 8, II, I), all traces of their junction being removed. Beneath these united folds there is therefore a cavity, within which the embryo lies (Fig. 8, $H, a e$ ). This carity is the cavity of the amnion. The folds which we have been describing are those which form the amnion.

Each fold, of course, necessarily consists of two limbs, both limbs consisting of epiblast and a very thin layer of mesoblast; but in one limb the epiblast looks towards the embryo, while in the other it looks away from it. The space between the two limbs of the fold, as can easily be scen in Fig. \&, is really part of the space between the somatopleure and splanchnopleure; it is therefore continuous with the general space, part of which afterwards becomes the pleuroperitoncal cavity of the body, shaded with dots in the figure and marked $(p p)$. So that it is possible to pass from the cavity between the two limbs of each fold of the amnion into the cavity which surrounds 
the alimentary canal. When the sereral folds meet and coalesce together above the embryo, they unite in such a way that all their inner limbs go to form a continuous inner membrane or sac, and all their outer limbs a similarly continuous outer membrane or sac. The inner membrane thus built up forms a completely closed sac round the body of the embryo, and is called the amniotic sac, or amnion proper, (Fig. S, H, I, \&c. a.), and the fluid which it afterwards contains is called the amniotic fluid, or liquor amnii. The space between the inner and outer sac, being formed by the united cavities of the several folds, is, from the mode of its formation, simply a part of the general cavity found everywhere between somatopleure and splanchnopleure. The outer sac over the embryo lies close under the vitelline membrane, while its periphery is gradually extended over the yolk as the somatopleuric investment of the yolk-sac described in the preceding paragraph.

9 . If the mode of origin of these two sacs (the inner or true amnion, and the outer or false amnion, as Baer called it) and their relations to the embryo be borne in mind, the reader will have no difficulty in understanding the course taken in its growth by an important organ, the allantois, of which we shall hereafter have to speak more in detail.

The allantois is fundamentally an appendage of the alimentary canal, and may be regarded as a bud thrown out by the splanchnopleure close to its junction with the somatopleure at the hinder end of the embryo (Fig. $8, D$, al.). From thence it grows first into the pleuroperitoneal cavity of the embryo, and thence very rapidly pushes its way by the development of a long stalk into the space between the true and false amniotic sacs (Fig. 8, G, $K$ ). Curving over the embryo, it comes to lie over the embryo and the amnion proper, separated from the shell (and vitelline membrane) by nothing more than the thin false amnion. In this position it performs its functions as a respiratory organ. It is evident that though now placed quite outside the embryo, the space in which it lies is a continuation of that peritoneal cavity in which it took its origin.

It is only necessary to add, that the false amnion either coalesces with the vitelline membrane, in contact with which 
it lies, or else replaces it, and in the later days of incubation is known as the chorion.

In the above account we have described the somatopleure as consisting of mesoblast as well as epiblast even in its most peripheral portions. The inner limbs of the anmiotic folds undoubtedly contain mesoblastic elements, since the amnion proper contains plain muscular fibres. Some authors however regard the outer limbs of the amniotic folds (giving rise to the false amnion) and the somatopleure beyond them as being composed of epiblast only. 


\section{CHAPTER III.}

THE CHAYGES WHICH TAKE PLACE DURING THE FIPST DAY OF INCUBATION.

1. During the descent of the egg along the oviduct, where it is exposed to a temperature of about $40^{\circ} \mathrm{C}$, the blastoderm, as we have seen, continues to undergo important changes. When the egg is laid and becomes cold these changes all but entirely cease, and the blastoderm remains inactive until, under the influence of the higher temperature of natural or artificial incubation, the vital activities of the germ are brought back into play, the arrested changes go on again, and usher in the series of events which we have now to describe in detail.

The condition of the blastoderm at the time when the egg is laid is not exactly the same in all eggs, in some the changes being farther advanced than in others, though the differences of course are slight; in some eggs, especially in warm weather, changes of the same kind as those caused by actual incubation may take place, to a certain extent, in the interval between laying and incubation; lastly, in all eggs, both under natural and especially under artificial incubation, the dates of the several changes are, within the limits of some hours, very uncertain, particularly in the first few days; one egg being found, for example, at 36 hours in the same stage as another at 24 or 30 hours, or a third at 40 or 48 hours. When we speak therefore of any event as taking place at any given hour or part of any given day, we are to be understood as meaning that such an event will generally be found to have taken place at about that time. We introduce exact dates for the convenience of description. 
The changes which take place during the first day will be most easily considered under three periods: from the 1st to the 12 th, from the 12 th to the 20 th, and from the 20 th to the 24th hour.

2. From the 1st to about the 12th hour.-During this period the blastoderm when viewed from above is found to have increased greatly in size. The pellucid area, which at the best is but obscurely marked in the unincubated egg, becomes very distinct (the central opacity having disappeared), and contrasts strongly with the opaque area, which has even still more increased both in distinctness and size.

For the first few hours both the pellucid and opaque areas remain circular, and the only change, besides increase in size and greater distinctness which can be observed in them, is a slight ill-defined opacity or loss of transparency, which makes its appearance in about the middle of the pellucid area. This is known as the embryonic shield.

3. Slight as are the changes which can at this stage be seen from surface views, sections taken from hardened specimens bring to light many most important changes in the nature and arrangement of the constituent cells.

It will be remembered that the blastoderm in the unincubated egg is composed of two layers, an upper (Fig. 3, ep.) and an under layer; that the upper is a coherent membrane of columnar nucleated ceils, but that the lower one (Fig. 3, $l$.) is formed of an irregular network of larger cells in which the nuclei, if present, are rarely visible; and that in addition to this there are certain still larger cells, called 'formative cells' (Fig. 3, b), lying at the bottom of the segmentationcavity.

Under the influence of incubation changes take place very rapidly, which result in the formation of the three layers of the blastoderm.

The upper layer, which we shall henceforward call the epiblast (Fig. 10, A), takes but little share in these changes.

In the lower layer, however, certain of the cells begin to get flattened horizontally, their granules become less numerous, and a distinct nucleus makes its appearance in them; the cells so altered cohere together and form a membrane (Fig. 10, C). The membrane thus formed, which is 
first completed in the centre of the pellucid area, we shall henceforward speak of as the hypoblast.

Between it and the epiblast many of the cells of the original lower layer are enclosed, and in addition some of the formative cells (migrating by belp of amœboid movements after the fashion of white-blood corpuscles) begin to travel round the edge of the hypoblast, and to pass in between it and the epiblast.

The cells, whether originally "formative" cells or cells from the lower layer, thus gathered between the epiblast and hypoblast, undergo a process of endogenous cell-formation, by which the whole of the interior of each becomes converted into a number of new cells. These new cells, spherical in form, and possessing a large nucleus with a distinct nucleolus, are first formed in the centre of the pellucid area and subsequently in its periphery. They constitute the third layer or mesoblast (Fig. 10, B).

The epiblast is the Hornblatt (corneal layer), and the hypoblast the Darmdriisenblatt (epithelial glandular layer) of the Germans, while those parts of the mesoblast which take part in the formation of the somatopleure and splanchnopleure correspond respectively to the Haut-muskel-platte and Larm-faser-platte.

All blood-vessels arise in the mesoblast. Hence the vascular layer of the older writers fall entirely within the mesoblast.

The serous layer of the same authors includes the whole of the epiblast, but also comprises a certain portion of mesoblast; for they speak of all the organs of animal life (skin, bones, muscle, \&c.) as being formed out of the serous layer, whereas the epiblast proper gives rise only to the epidermis and to certain parts of the nervous systern. In the same way their mucous layer corresponds to the hypoblast with so much of the mesoblast as takes part in the formation of the organs of organic life. Their vascular luyer therefore answers to a part only of the mesoblast, viz. that part in which blood-vessels are especially develıped.

It is worthy of notice that the cells of the epiblast are themselves the direct results of segmentation; but that the hypoblast and mesoblast are formed at a subsequent period, and are therefore only indirectly the results of segmentation. The true difference between the hypoblast and mesoblast lies in the mode in which each layer is formed, and not in any essential difference in the segmentation-spheres from which each is derived.

At about the time when the hypoblast is completely formed as a distinct membrane, the mesoblast cells form a somewhat thick mass in the centre of the blastoderm, and cause the central opacity spoken of above as the embryonic shield.

4. Soon after this, between the 8 th and 12th hours, the hitherto circular pellucid area becomes oval (the opaque area remaining circular). The oval is, with remarkable regu- 
larity, so placed that its long axis forms a right angle, or very nearly a right angle, with the long axis of the egg itself. Its narrow end corresponds with the future hind end of the embryo: and we may henceforward speak of it as the hind end. If an egg be placed with its broad end to the right hand of the observer, the head of the embryo will in nearly all cases be found pointing away from him.

At about the time when the pellucid area is beginning to undergo this change of shape, there appears in surface views, along a line corresponding with the long axis of the oval, and occupying not, as might perhaps be expected, its front but its hinder two-thirds, a narrow opaque streak, much more opaque, and therefore distinct, than the embryonic shield, but still shadowy and ill-defined. This is known as the primitive streak.

Fig. Io.

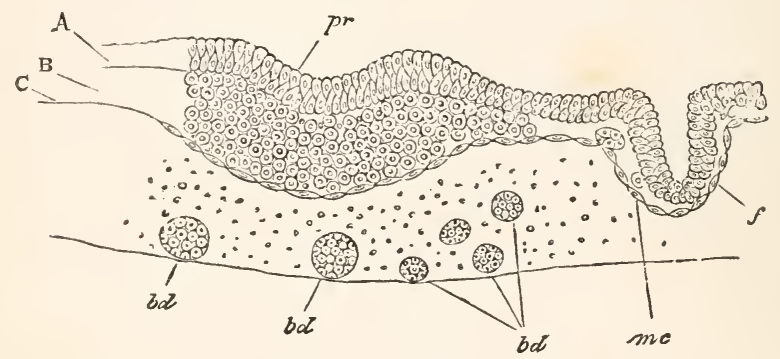

Section of a Blastoderm at right $\Lambda$ Ngles to the LoNg axis of the Embryo after eight hours' Incubation.

(Taken about midway between front and hind end.)

A. epiblast. B. mesoblast. $C$. hypoblast. $p r$. primitive groove. $f$. fold in the blastoderm, probably produced by the action of the chromic acid. m. c. mesoblast cell; the line points to one of the peripheral mesoblast cells lying between epiblast and hypoblast. $b d$. formative cells.

The following are the chief points represented in the section. (1) The thickening of the mesoblast underneath the primitive groove $p^{2}$., even when it is hardly at all present at the sides of the groove. (2) The hypoblast, $C$, early formed as a single layer of spindle-shaped cells. (3) The so-called segmentationcavity, in which coagulated albumen is present. On the floor of this are the large formative cells $b d$.

The line of separation between the epiblast and mesoblast underneath the primitive groove is too strongly marked in the figure.

The primitive streak is no sooner formed than it becomes marked on its upper surface by a delicate shallow furrow 
running along its axis. In fresh specimens viewed with transmitted light, this furrow appears as a linear transparency, but in hardened specimens seen under reflected light may be distinctly recognized as a furrow or narrow groove, the bottom of which being thinner than the sides appears more transparent when viewed with transmitted light. It is known as the primitive groove. The nature of the changes by which it is brought about can only be learnt by the study of vertical sections (Fig. 10). These teach us that the opacity which marks out the primitive streak is chiefly due to a thickening of the mesoblast. In the formation, however, both of the primitive streak, and especially of the primitive groove, the epiblast also plays an important part.

During these twelve hours the epiblast has been spreading rapidly, much more rapidly than the other two layers. Over the white yolk in the region of the opaque area it forms a layer one cell deep, but at the same time has become two or three cells deep in the centre of the pellucid area. In the pellucid area its constituent cells have become narrower $(6 \mu)$ and more columnar, but over the opaque area flatter and broader $(12 \mu)$ than they were at first. At the 12th hour therefore we find a distinct histological difference between the epiblast cells of the pellucid and those of the opaque area.

Over the thickening of the mesoblast, which forms the basis of the primitive streak, the epiblast is also thickened; the hypoblast, however, remains here, as in the rest of the blastoderm, a flat sheet consisting of a single layer of flattened (seen in sections as a single row (Fig. 10, C) of spindleshaped) cells, which become larger and more irregular at the periphery. The thickening of the mesoblast and epiblast in the region of the primitive streak causes the upper outline of the blastoderm as seen in sections to rise above the general surface in a gentle curve (Fig. 10).

The primitive groove is formed almost entirely by a pushing in or depression of the epiblast at the summit of this curve.

The thickness of the epiblast remains about the same on the sides as at the bottom of the groove. The mesoblast, on the contrary, is thinner immediately beneath the bottom of the groore than at the two sides, where it is decidedly 
thicker than in the rest of the pellucid area. It is apparently this median thinning of the mesoblast which gives rise to the linear transparency seen in specimens viewed with transmitted light. The hypoblast, it may be remarked, is generally curved downwards beneath the primitive streak and groove, though not to the same extent as the epiblast. Thus the whole blastoderm is somewhat curved in this region. Immediately beneath the groove a kind of fusion takes place between the epiblast and mesoblast, though on close examination the line of junction between them can generally be made out. This apparent fusion His (Ueber die Erste Anlage des Wirbeltheirlcils) regarded as an event of great importance, and gave the name of axis-cord to the part in which it occurs. In fresh specimens a narrow (opaque) streak can be seen running down the centre of the groove; but it is not represented by any structure which can be seen in sections.

The chief events then which occur during the first twelve hours of incubation are the establishment of the three layers of the blastoderm, and the appearance of the embryonic shield, of the primitive streak and of the primitive groove.

5. From the 12th to the 20th hour.-During this period the pellucid area rapidly increases in size, and from being oval becomes pear-shaped. The primitive groove grows even more rapidly than the pellucid area; so that by the 16 th hour it is not only absolutely, but also relatively to the pellucid area, longer than it was at the 12th hour. 'The interval between its end and the circumference of the pellucid area continues to be greater in front than behind.

At about the 16th hour, or a little later, a thickening of the mesoblast takes place in front of the primitive groove, giving rise to an opaque streak ending abruptly in front against a semicircular fold, which appears at this time near the anterior extremity of the pellucid area (Fig. 11), and is known as the head-fold. In fresh specimens this streak looks like a continuation from the anterior extremity of the primitive groove; but in hardened specimens it is easy to see that the connection is only an apparent one.

Along the new streak a groove (Fig. 11, m.c.) is very soon formed, which, narrow in front, but widening very much behind, embraces between its diverging walls the anterior extremity of the primitive groove. 'This new groore, by the conversion of which into a tube the medullary canal will be formed, is known as the medullary groove.

On each side of it the mesoblast is thickened, and the surface of the blastoderm raised up in the form of two longitudinal folds, known as the lamince dorsales, or the medullary folds (Fig. 11, A). Immediately beneath the bottom of the 
FIG. II.

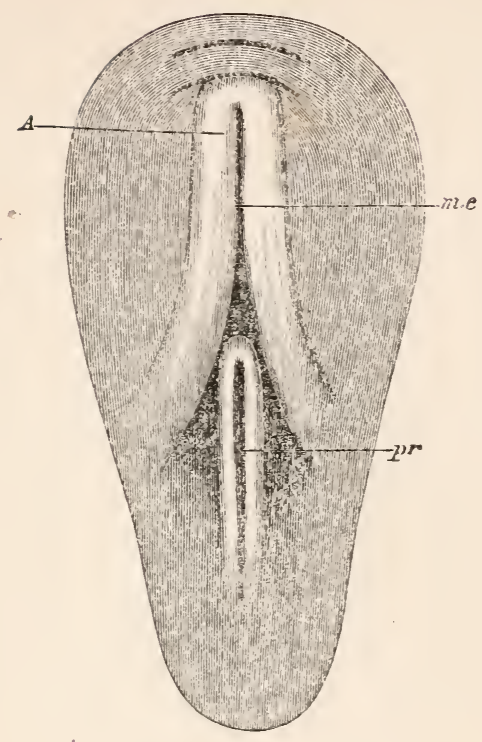

Surface View of the Pellocid Area of a Blastodery of i 8 hours.

None of the opaque area is shewn, the pear-shaped outline indicating the limits of the pellucid area.

At the hinder part of the area is seen the primitive groove $m$., with its nearly parallel walls, fading away behind, but curving round and meeting in front so as to form a distinct anterior termination to the groove, about half way up the pellucid area.

Above the primitive groove is seen the medullary groove $m . c$, with the medullary folds $A$. These diverging behind, slope away on either side of the primitive groove, while in front they curve round and meet each other close upon a curved line which represents the head-fold.

The second curved line in front of and concentric with the first is the commencing fold of the amnion.

groove, however, the mesoblast is thinned out and very soon the cells in this position, separating from the lateral masses, adhere together in the middle line, and thus form between the epiblast and the hypoblast a flattened circular rod known as the notochord, seen in section as an elliptical aggregation of cells (Fig. 12, ch.) 
The medullary groove differs in many important particulars from the prinitive groove. Beneath the primitive groove the mesoblast always fuses more or less with the epiblast; this is never the case under the medullary groove. Under the primitive groove the mesoblast never shews any signs of differentiation into any organ; under the medullary groove the notochord is formed out of the mesoblast cells. The epiblast lining the bottom of the medullary groove frequently becomes very much thinner than at its sides; this seems never to be the case with the primitive groove.

The primitive groove reaches its maximum growth before the appearance of the medullary groove; and after the appearance of the latter gradually becomes less and less conspicuous, and finally disappears without leaving a trace. A curved remnant of it is to be found at the hind end of the medullary canal between the 3 oth and 4 oth hours, but by the 5oth not a trace of it remains.

By the earlier observers the primitive groove was supposed to become converted into the medullary canal. Dursy (Der Primitivstreif des Hühnchens) was the first to give a correct account of its disappearance; and the distinction letween it and the medullary groove has since been fully recognized by many ohservers. Goette (Archiv. Micr. Anat. Vol. x. 1873, pp. 145-199) describes the medullary groove as always appearing to the left of the primitive groove, and having its floor continuous with the left wall of the latter. He states that beneath this left wall the unsvmmetrically placed axis-cord is found; indeed he considers that the notochord is a forward continuation of the axis-cord, and that the latter, as the primitive groove recedes befure the medullary groove, becomes continuously converted into the former.

The primitive groove theu is a structure which appears early, and son disappears without entering directly into the formation of any part of the future animal. Apparently it has no function whatever. We can only supjos s that it is the ruliment of some ancestral feature.

6. By the 20th hour the medullary groove or canal, with its medullary folds or laminæ dorsales, is fully established. It then presents the appearance, towards the hinder extremity of the embryo, of a shallow groove with sloping diverging walls which embrace between them the remains of the vanishing primitive groove.

Passing forwards towards what will become the head of the embryo the groove becomes narrower and deeper with steeper walls. On reaching the head-fold (Fig. 11), which continually becomes more and more prominent, the medullary folds curve round and meet each other in the middle line, so as to form a somewhat rounded end to the groove. In front therefore the canal does not become lost by the gradual flattening and divergence of its walls as is the case behind, but has a definite termination, the limit being marked by the head-fold.

In front of the head-fold, quite out of the region of the medullary folds, there is usually another small fold which is the beginning of the ammion (Fig. 11). 
We must now go back, and say a few words about the changes which the cells of the various layers undergo from the $12-20$ hours.

FIG. 12.

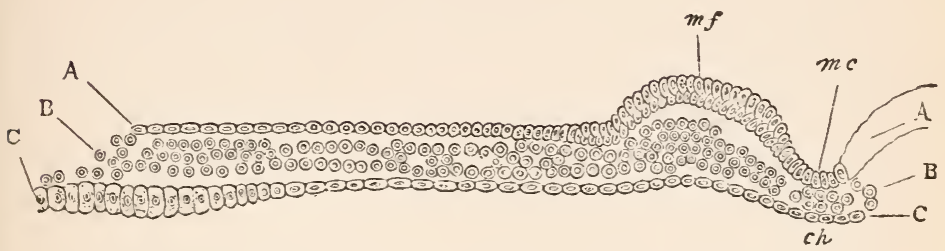

Traxsferse Secrion of a Blastodery ixcubated for i 8 hours.

The section passes through the medullary groove mc, at some distance behind its extreme front, and shews some of the chief puints in which it differs frum the primitive groove.

The chief of these are, (I) the presence underneath it of the notochord ch., (2) the absence of any apparent adhesion between the epiblast and the mesoblast, (3) the thickening of the mesoblast underneath the medullary folds, $m f$.

$A$. epiblast. B. mesoblast. C. hypoblast.

m. c. medullary groove. m. $f$. medullary fold. ch. notochord: the small group of mesoblast cells separated by a narrow gap from the thicker mass of mesoblast on either side.

It is to be noticed that the cells of the hypoblast become more columnar as they approach the edge of the pellucid area, and finally pass, without any strong line of demarcation, into the white-rolk spheres.

Only one half of the section is represented-if completed the section would be symmetrical about the line passing through the centre of the medulary canal, $m c$.

The hypoblast (Fig. 12, $C$ ) continues to be only one cell deep; the cells being, during the whole of this period, flatter in the centre, and larger and more irregular towards the periphery of the blastoderm. At about the 12th hour they are very irregular in size; shewing very great variations over a very small space. This probably implies that they are rapidly undergoing division. Later, however (about the 1sth hour), they are fairly uniform over particular regions, though they vary considerably in size at different parts of the pellucid area. In no case does the hypoblast extend beyoud the edge of the pellucid area.

The hypoblast cells along the central axis of the pellucid area, and for some little distance on each side, are smaller than elsewhere over the blastoderm. Over a small district just outside the embryo, and at about one-third of the way from the posterior extremity of the blastoderm, they are, from the I 8 th-23rd hour, considerably larger than anywhere else. The remaining hypoblast celis 
are intermediate in size between these very large cells and the smaller cells in the centre. During the whole of this period the hypoblast cells continue to be granular and filled with highly refractive spherules, exhibiting in this respect a marked contrast to their appearance at a it ter time.

Their mode of increase is partly by division, but the layer grows chiefly in a manner which is very different and somewhat remarkable. Eefore the I 2 th hour the hypoblast at its margin ended abruptly against the white-yolk cells; but after that hour its relation to the white yo.k becomes altered. As they approach the white yolk the cells of the hypoblast become more and more filled with white-yolk spherules, and at the extreme edge of the pellucid area it is very difficult to say where the white yolk ends, and where the hypoblast begins. This is somewhat diagrammatically shewn in Fig. 12. The white-yolk spheres near the edge of the pellucid area have generaly acquired nuclei, though it is frequently difficult to see them owing to the numerous highly refractive spherules which the spheres contain. The nearer they are to the edge of the pellucid area the fewer spherules they contain, and at the very edge it is almost impossible to say whether they ought to be called whitevolk spheres or hypoliast cells. The chief increase of the hypoblast therefore seems to take place through the couversion, cell for cell, of the white yolk into the hypoblast.

During this period the mesoblast (Fig. 12, $B$ ) cells do not undergo any marked change. The layer itself enlarges to a certain extent through the multiplication of cells by the division of old ones; but the chief increase in bulk is probably due to the formative cells, which are continually passing round from the bottom of the segmentation-cavity to the mesoblast, and there become converted, in the way described ( $\$$ 3.) above, into mesoblast cells.

These formative cells are more numerous at the bottom of the segmentationcavity at the 18 th hour than they were at the first hour. This accession to their number is probably due to fresh ones being formed from the floor of white yolk. They appear to grow in size by absorbing the white-yolk spherules, with which indeed they are completely filled.

The cpiblast cells (Fig. 12, A) probably increase entirely by division, and seem to derive their nourishment from the white yolk on which the peripheral cells rest, and perhaps also from the albuminous fluid which fills the segmentationcavity and occupies all the interstices between the cells of the various layers.

The cells near the edge of the opaque area are the largest and flattest of the epiblast cells; those in the middle of the pellucid area are smaller than those at its edge.

Ontside the blastoderm there are to be seen on the surface of the yolk alternating transparent and opaque white rings. These are known as the halonss, and frequently appear at the commencement of incubation. It is stated by His that they are to be explained by the white-yolk spheres undergoing changes of two kinds. In the one case the spherules they contain are 
dissolved and give place to vacun!es; where this occurs to a large extent an opactue ring is firmed. In the other case a solution of the protoplasm of the spheres takes place, and the splserules are let loose in large numbers; where this occurs a transparent ring is formed.

The chief events then of the second part of the first day, are the appearance of the medullary folds and groove, the formation of the notochord, the beginning of the head-fold and amnion, and the histological changes taking place in the several lavers.

7. From the 20th to the 24th hour. The head-fold enlarges rapidly, the crescentic groove becoming deeper, while at the same time the overhanging margin of the groove (the upper limb of the 2 , Chap. II. $\$ 5)$, rises up above the level of the blastoderm; in fact, the formation of the head of the embryo may now be said to have detinitely begun.

8 . The medullary folds, increasing in size in every dimension, but especially in height, lean over from either side towards the middle line, and thus tend more and more to roof in the medullary canal, especially near the head. About the end of the first day they come into direct contact and completely coalesce with each other at a point which lies at some little distance behind the head-fold, in the region which will afterwards become the neck. Union, having begun at this spot, rapidly runs forward till (early in the second day) the head-part is completely closed in ; and then passes more slowly backwards. The whole of the anterior portion of the groove is closed in before the union has advanced more than a very short distance towards the tail. In this way a tubular canal is former, ending blindly in front, but as yet open behind. This is the medullary or neural canal (Fig. 13, M, Fig. 20, MC.). It is not completely closed in at the tail till a period considerably later than the one we are considering.

9. Meanwhile important changes are taking place in the axial portions of the mesoblast, which lie on each side of the notochord beneath the medullary folds.

In an embryo of the middle period of this day, examined with transmitted light, the notochord is seen at the bottom of the medullary groove between the medullary folds, as a trarsparent line shining through the floor of the groove when the embryo is viewed from above. On either side of the 
notochord the body of the embryo appears somewhat opaque, owing to the thickness of the medullary folds; as these folds slope away outwards on either side, so the opacity gradually fades away in the pellucid area. There is present at the sides no sharp line of demarcation between the body of the embryo and the rest of the area; nor will there be any till the lateral folds make their appearance; and transverse vertical sections shew (Fig. 12) that there is no break in the mesoblast, from the notochord to the margin of the pellucid area, but only a gradual thinning.

10. During the latter period of the day, however, the plates of inesoblast on either side of the notochord begin to be split horizontally into two layers, the one of which attaching itself to the epiblast, forms with it the somatopleure (Fig. 13, compare also Fig. 2(), So.), while the other, attaching itself to the hypoblast, forms with it the splanchnopleure (Fig. 13, $B c, \mathrm{Fig} .20, s p)$. By the separation of these two layers from each other, a cavity (Fig. 1., $p p$, and Fig. 20, pl), containing fluid only, and more conspicuous in certain parts of the embryo than in others, is developed. This cavity is the beginning of that great serous cavity of the body which afterwards becomes divided into separate cavities. We shall speak of it as the pleuro-peritoneal cavity.

11. This cleavage into somatopleure and splanchnopleure does not extend quite up to the walls of the medullary canal. Hence there is left along either side of the canal, between it and the line along which the cleavage begins, a tract or plate of uncleft mesoblast, which receives the name of vertebral plate, the more external mesoblast being called the lateral plate.

At first each vertebral plate is not only unbroken along its length but also continuous at its onter edge with the upper and lower layers of the lateral plate of the same side. Very soon, however, clear transverse lines are seen, in surface views, stretching inwards across each vertebral plate from the lateral plate towards the notochord; and not long after a transparent longitudinal line makes its appearance on either side of the notochord along the line of junction of the lateral with the vertebral plate.

These transparent lines are caused by the appearance of vertical clefts, giving rise to narrow spaces containing nothing 
but clear fluid; and transverse sections shew that they are due to breaches of continuity in the mesoblast only, the epiblast and hypoblast having no share in the matter. The first transverse lines which appear are two in number, one a little behind the other, about opposite the spot where the medullary folds first coalesced to form the neural tube. The longitudinal lines begin at about the same place and run thence backward, parallel to the notochord, as far as the closure of the medullary canal extends. Behind the first two transverse lines other parallel transverse lines in course of time make their appearance.

Thus each vertebral plate appears in surface views to be cut up into a series of square plots, bounded by transparent lines. Each square plot is the surface of a corresponding cubical mass (Fig. 13, P. v., Fig. 20, P. v.). The two such

\section{FIG. I3.}

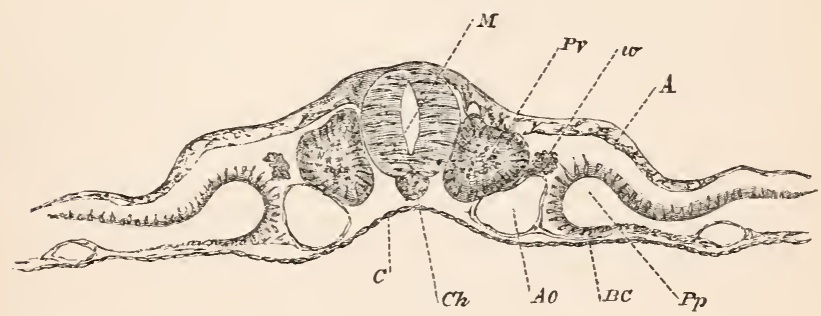

Transverse Section throdgh the Dorsal Region of an Embryo of the SEcoxd DAY. (Copied from His), introduced here to illustrate the formation of the protovertebræ, and the cleavage of the mesoblast.

The provertebræ appear irregularly quadrate; they woull have been more distinctly square, had the section been one of the first day. before the appearance of the primitive aortæ, and of the rudiments of the Wolffian ducts: cumpare Fig. 20.

M. medullary canal. $P v$. protovertebra. $w$. rudiment of Wolffian duct. A. epiblast. C. hypoblast. Ch. notochord. Ao. aorta. $B C$. splanchnopleure.

cubical masses first formed, lying one on either side of the notochord beneath, and a little to the outside of the medullary folds, are the first pair of protovertebrce. Behind this first pair, but otherwise similarly situated, a second and third pair make their appearance during the first day. 
The vertebral plate, while still continuous with the lateral plate (the distinction between the two being indicated solely by the cleavage of the latter), consists of several layers of cells ; but of these only the uppermost layer, that immediately under the epiblast, appears to be continued into the somatopleure; the whole of the remainder, including those cells which will eventually form the so-called nucleus of the protovertebræ, seem to pass directly into the splanchnopleure.

All these changes except the formation of the pleuro-peritoneal cavity can be seen in surface views of fresh transparent specimens, but their nature is best shewn in sections.

12. Since the commencement of incubation the area opaca has been spreading outwards over the surface of the yolk, and by the end of the first day has reached about the diameter of a sixpence. It appears more or less mottled over the greater part of its extent, but this is more particularly the case with the portion lying next to the pellucid area; so much so, that around the pellucid area an inner ring of the opaque area may be distinguished from the rest by the difference of its aspect.

At about the 20th-24th hours an increasing number of formative cells make their way from the segmentation-cavity to the edge of the area opaca, and there, immediately underneath the epiblast, quickly become converted into a rather thick and somewhat irregular network of mesoblast cells. 'The mottled appearance of the inner ring spoken of above is due to changes taking place in this mass of mesoblast, changes which eventually result in the formation of what is called the vascular area, the outer border of which marks the extreme limit to which the mesoblast extends.

During the whole of this period the medullary groove has been growing rapidly backwards, so that the primitive groove appears to be pushed further and further back, and at the same time becomes smaller and less conspicuous. The amniotic fold is at the end of the first lay very noticeable.

13. The changes then which occur during the first day may thus be briefly summarized:

(1) 'The hypoblast and mesoblast are formed from the segmentation-spheres, so that by the 6 th to the 8 th hour the three layers of the germ-the epiblast, the mesoblast, and the hypoblast-are definitely established.

(2) The primitive streak is formed by a thickening of the mesoblast. 
(3) The primitive groove is formed along the centre of the primitive streak.

(4) The pellucid area becomes pear-shaped, the broad end corresponding with the future head of the embryo. Its long axis lies at right angles to the long axis of the egg.

(5) The medullary groove makes its appearance in front of the primitive groove, and below it the notochord is formed out of mesoblastic cells.

(6) The development of the head-fold gives rise to the first definite appearance of the head.

(7) The medullary folds rise up and coalesce in the region of the neck to form the neural tube, the primitive streak and groove disappearing. pearance.

(8) One or more pair of protovertebrce make their ap-

(9) By the cleavage of the mesoblast, the somutopleure separates from the splunchnopleure. the head-fold.

(10) The first trace of the amnion appears in front of

(11) The vascular area begins to be be distinguished from the rest of the opaque area.

It may be well to remark, before passing on to the second day, that out of the protovertebre are formed not only the permanent vertebræ, but also the superticial dorsal as well as certain other muscles and the spinal nerves; that the pair of protovertebræ first formed corresponds not with the first cervical vertebra of the adult chick, but rather with the third or even fourth; for though the majority of the protorertebræ are formed regularly behind the first pair, two or even three pair may make their appearance in front of it; and lastly, that in the part of embryo which forms the head, the mesoblast is never cut up into protovertebræ, and never undergoes cleavage to form somatopleure and splanchnopleure. 


\section{CHAPTER IV.}

THE CHANGES WHICH TAKE PLACE DURING THE SECOND DAY.

1. The First Half of the Second Day. In attempting to remove the blastoderm from an egg which has undergone from 30 to 36 hours' incubation, the observer cannot fail to notice a marked change in the consistency of the blastodermic structures. The excessive delicacy and softness of texture which rendered the extraction of an 18 or 20 hours' blastoderm so difficult, has given place to a considerable amount of firmness; the outlines of the embryo and its appendages are much bolder and more distinct; and the whole blastoderm can be removed from the egg with much greater ease.

In the embryo itself viewed from above one of the features which first attracts attention is the progress in the head-fold (Fig. 15). The upper limb or head has become much more prominent, while the lower groove is not only proportionately deeper, but is also being carried back beneath the body of the embryo (Chap. II. §. 5.)

2. The medullary folds are closing rapidly. In the region of the head they have quite coaleseed, a slight notch in the michlle line at the extreme front marking for some little time their line of junction. The open medullary groove of the first day has thus become converted into a tube, the neural canal, closed in front, but as yet open behind. For a brief period the calibre of this tube is uniform throughout; but very speedily the front end dilates into a small bulb, whose cavity remains continuous with the rest of the neural canal, and whose walls, like those of the canal, are formed of epiblast. This bulb is known as the first cerebral vesicle, Fig. 14, $F B$, 
FIG. I4.

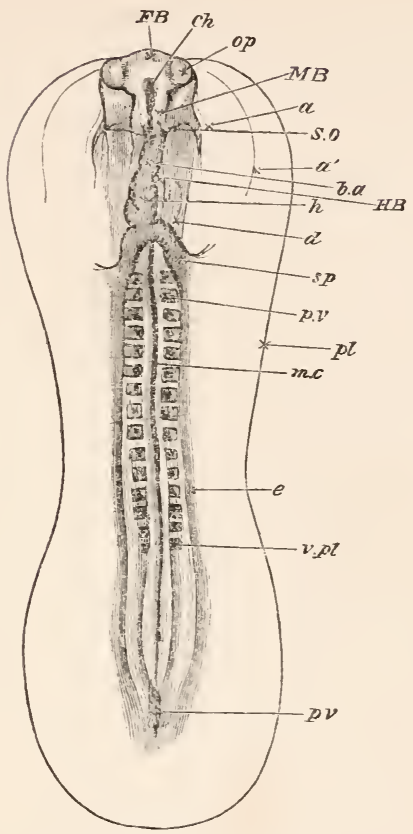

An Eybrto Chick of the first day (About thitti-six hours) viewed FROM BELOW AS A TRANSPARENT OBJECT.

$p l$. Outline of the pellucid area.

$F B$. The forebrain or first cerebral vesicle, projecting from the sides of which are seen the optic vesicles op. A definite head is now constituted, the backward limit of the somatopleure fold being indicated by the faint line $S . O$. Around the head are seen the two limbs of the amniotic headfuld: one, the true amuion $a$, closely enveloping the hear, the other, the false amnion $a^{\prime}$, at sone distance from it. The head is seen to project beyond the anterior limit of the pellucid area.

The splanchnopleure fold extends as far back as sp. Along its diverging limbs are seen the conspicunus venous root; or omphalo-mesaraic veins, uniting to form the heart $h$, which continuing forward as the bulbus arteriosus $b a$, is lost in the substance of head just in tront of the somatop'eure fold.

Lying (in this position of the embryo) under the heart is seen the broad foregut $d$, the wide crescentic opening into which at the hind linit of the splanchnopleure fold is very conspicuons. Beneath the foregut are faintly seen the hind brain $H B$. and higher up and more distinctly the mid brain $M B$. These are not yet completely differentiated, and their limits are in consequence very obscurely indicated. 
Behind the splanchnopleure fo'd, marking the hind limits of the foregut, are seen the two rows of protovertebia, the dark line between which $m$. $c$. indicates the position both of the line of junction of the medullary folds and of the notochord. The front end of the notochord is seen at ch. underneath the forebrain; its hind end is indistinct. Towards the tail the protovertebræ become indistinct and give place to the vertebral plates $v . p /$. Still further back, at the commencing tail, all the parts become indistinct, the remains of the primitive groove $p w$. being as conspicuous as anything else.

and makes its appearance in the early hours of the second day. Behind it a second and a third bulb, the second and third cerebral vesicles, are successively formed in a similar manner; but the consideration of these, though they begin to make their appearance soon after the formation of the first cerebral vesicle, may be conveniently reserved to a later period.

3. The number of protovertebræ increases rapidly. The one or two pairs which are seen at the end of the first day have by the middle of the second day multiplied to five, or eight, or even more, Figs. 14, 15, p.v', each being formed in the same way as the first. As was mentioned previously, the chief increase takes place from before backwards, the new protovertebrx appearing behind the old ones; but one pair at least is probably formed in front of that which was the very first to appear.

In the early part of this day the formation of new protovertebra keeps pace with the closing in of the melullary folds, so that that part of the canal which is already closed in is always flanked by protovertebra; but later on the formation of protovertebre lags behind, so that for some distance towards the hinder extremity the closed-medullary canal is unprotected by protovertebr: Fig. 15. At the extreme end the merlullary folds become shallower, diverge from each other, and afterwards meet again, thus forming a lozengeshaped open depression known as the sinus rhomboidalis, Fig. 15, s. $r$.

Behind the sinus rhombnilalis there may generally be seen a small and usually curved remnant of the primitive groove. Fig. $15, p . r$.

4. In a former chapter it was pointed out (Chap. II. \$ 5) that the embryo is virtually formed by a folding or tucking in of the limited portion of the blastoderm, first at the anterior extremity, and afterwards at the posterior extremity and at 


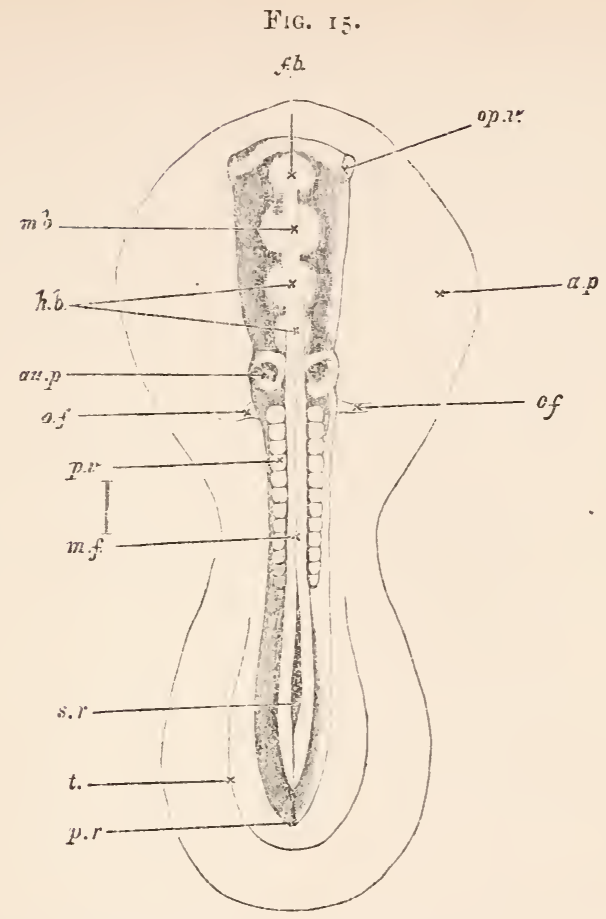

Esibrio of the Chick at 36 hodrs Vietred ffioj a OBJECT. (Chromic acid preparation.)

$f . b$. front brain. m. $b$. mid brain. h. $b$. hind brain. op. $v$. optic resicle. au. $p$. auditory vesicle. o. f. omphalo-mesaraic vein. $p . r$. protovertebra. m. $f$. line of junction of the medullary folds above the merlullary canal. $s . r$. sinus rhomboldalis. $t$. tail-fold. $p . r$. remains of primitive groove. a. $p$. area pellucida.

The line to the side between $p . v$. and $m$. $f$. represents the true length of the embryo.

Tne biscuit-shaped outline indicates the margin of the pollucid area. The head, which reaches as far back as o.f, is distinctly marked off; but neither the somatopleuric nor splanchnopleuric folds are shewn in the figure; the latter diverge at the level of $o . f$, the fcrmer considerably nearer the front, somewhere between the lines $m . b$. and $h . b$. The optic vesicles $o r . v$. are seen bulging out beneath the superficial epiblast. The heart lying underneath the opaque body cannot be seen. The tail-fold, $t$., is just indicated; no distinct lateral folds are as yet visible in the region midway between hear and tail. At $m . f$. the line of junction between the medullary folds is still visible, being lost forwards over the cerebral vesicles, while behind the folds diverge to enclose the narrowing sinus rhomboidalis, $s . r$. 
the sides. One of the results of this doubling up of the blastoderm to form the head is the appearance, below the anterior extremity of the medullary tube, of a short canal, ending blindly in front, but open widely behind (Fig. 16, I)), a cul de sac in fact, lined with hypoblast reaching from the extreme front of the embryo to the point where the splanchnopleuric leaf of the head-fold (Fig. 16, $F . S p$ ) turns back on itself. This cul de sac, which of course becomes longer and longer the farther back the hear-fold is carried, is the rudiment of the front end of the alimentary canml, the foregut, as it might be called. In transverse section it appears to be flattened horizontally, and also bent, so as to have its convex surface looking downwards, (Fig. $18 \mathrm{al}$ ). At first the anterior end is quite blind, there being no mouth at all; the formation of this at a subsequent date will be described later on.

At the end of the first half of the second day the head-

Fig. I6.

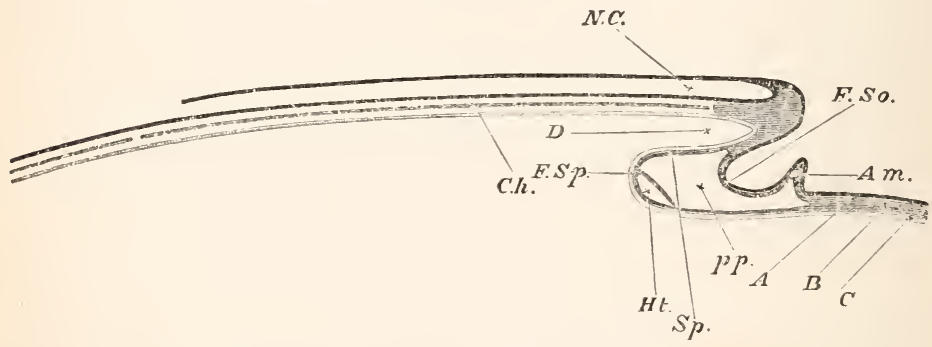

Diagramisitic Loxgitudinal Section throdgh the Axis of an Embryo.

The section is supposed to be made at a time when the head-fold has commenced but the tail-fold has not yet ilppeared.

N. C. neural canal, closed in front but as yet open behind. Ch. nntochord, not reaching to the extreme front, snd not as yet fully formed behind. The section being talsen in the niddle line, the protovertebre are of course not shewn. In front of the notochord is seen a mass of uncleft mesoblast, which will eventually form part of the skull. $D$. the commencing foregut or front part of the alimentary canal. F. So. Somatopleure, raised up in its peripheral portion into the anmiotic fold $A \mathrm{~m}$. Sp. Splanchnopleure. At $S_{p}$. it forms the under wall of the foregut; at $F$. Sp. it is turning round and about to run forward. Just at its turning point the cavity of the heart $H t$ is being developer in its mesollast. $\quad p p$. pleuroperitoneal cavity. $A$ epiblast, $B$ mesoblast, $C$ hypotlast, indicated in the rest of the figure by differences in the shading. At the part where these three lines of reference end the mesoblast is as yet uncleft. 
fold has not proceeded very far backwards, and its limits can easily be seen in the fresh embryo both from above and from below.

5. It is in the head-fold that the formation of the heart takes place, its mode of origin being connected with that cleavage of the mesoblast and consequent formation of splanchnopleure and somatopleure of which we have already spoken.

At the extreme end of the embryo (Fig. 16), where the blastoderm begins to be folded back, the mesoblast is never cleft, and here consequently there is neither somatopleure nor splanchnopleure; but at a point a very little further back, close under the blind end of the foregut, the cleavage (at the stage of which we are speaking) begins, and the somatopleure, $F$. So, and splanchnopleure, $F$. $S p$. diverge from each other. They thus enclose between them a cavity, $p$ ), which rapidly increases behind by reason of the fact that the fold of the splanchnopleure is carried on towards the hinder extremity of the embryo considerably in advance of that of the somatopleure. Both folds, after running a certain distance towards the hind end of the embryo, are turned round again, and then course once more forwards over the yolk-sac. As they thus return (the somatopleure having meanwhile given off the fold of the ammion, $A m$.), they are united again to form the uncleft blastodermic investment of the yolk-sac. In this way the cavity arising from their separation is closed below.

It is in this cavity, which from its mode of formation the reader will recognise as a part (and indeed at this epoch it constitutes the greater part) of the general pleuroperitoneal cavity, that the heart is formed.

It makes its appearance at the under surface and hind end of the foregut just where the splanchnopleure folds turn round to pursue a forward course, (Fig. 16, Ht); and by the end of the first half of the second day (Fig. 14, h) has acquired somewhat the form of a flask with a slight bend to the right. At its anterior end a slight swelling marks the future bulbus arteriosus; and a bulging behind indicates the position of the auricles. It is hollow, and its cavity opens below and behind into two vessels called the omphalo-mesaraic veins (Figs. 14, 15, o.f), which pass outwards in the folds of the splanchnopleure at nearly right angles to the axis of the 
embryo. The anterior extremity of the heart is connected with the two aortæ.

The muscular portion of the walls of the heart are derived in the chick (as in all other vertebrates in which the point has been worked out) from the mesoblast of the sp'anchnopleure.

A though thus much may be asserted with tolerable certainty for all vertebrates; yet the exact node of development appears, according to our present knowledge, to be very different in different cases; and it seems probable that these differences are in part the result of variations in the mode of formation and time of closure of the alimentary canal.

In the chick tie investigation of the earlier stages of the heart is beset with considerable difficulties; and accordingly various inquirers have arrived at very different results, though thie majority are agreed as to its formation from the mesoblast of the splanchnopleure. Exact information concerning the epithelium lining the heart may be said to be almost completely wanting.

Von Baer described the heart as consisting in its earliest stage of two solid ascregations of the mesoblast cells of the splanchnopleure, converging in front at the end of the foregut where they are loosely united together by a thin band, but diverging behind along the diverging folds of the splanchnopleure. As the foregut lengthens, the two masses coalesce more and more completely in front, until the whole structure assumes the shape of a fusiform mass, attached to the under wall of the foregut, with prolongations stretching like the limbs of an inverted $X$ along the folds of the splanchnopleure on either side. At first solid throughout, the $\mathrm{X}$-shape mass subsequently becomes hollowed ont and filler with fluid by the solution of its central cells.

The account given by Remak (Entuickelung der Wirbelthicre, I855) is some. what similar.

According to His the beart is formed by the separation of a layer.of the splanchnopleure and its coalescence with a similar layer from the somatopleure. It is therefore from the beginning hollow. Its cavity is also from the beginning continuous with the canals of the aortæe and omphalo-mesaraic veins, the roots of which are formed in a precisely similar manner as itself. It is through these that the epithelial (endothelial) elements, derived from the white yolk, make their way into the heart to form its epithelial (endothelial) lining.

According to Afanassieff (Bull. A cad. st. Pétershourg., Tom. xuI. i \$69, pp. $32 \mathrm{I}-335)$ the heart is formed by the longitudinal separation of a thick layer of the mesoblast of the splanchnopleure along the under wall of the foregnit. At either side, so much of the mesoblast is detached, that only a single layer of cells (seen spindle-shaped in a transverse section of the embryo) remains united with the hypoblast to form the wall of the gut. Along the middle line the separation is not complete, the detached layer of mesollast being here still connected with the wall of the gut by a few cells. The single layer of spindleshaped cells breaks loose in turn from the wall of the gut on either side of the middle line, but still remains attached along the middle line itself. We have thins in transverse section a thimner and a thicker layer of mesoblast, hanging down in a double festoon from the (hypoblastic) under-wall of the gut. Both linyers become more and more separated from the gut, and bulge out into the pleuroperitoneal space, thus creating between themselves and the gut a cavity, which is at first double, but, by the clisappearance of the ceils along the middle line, subsequently becomes single. This is the cavity of the heart, the thick layer representing its muscular walls, and the thin its epithelial lining. The two ends are open, the hinder end being connected with the omphalo-mesaraic veins, and the front with the aortæ. At first the heart is not a tube with 
complete walls of its own, but rather a cavity, closed in below and at the siles by its mesoblastic walls, and roofed over by the bare hypoblastic under-wall of the foregut. Very shortly, however, the side walls cluse in above, and thus pinch off the heart as a complete and distinct tube, which becomes quite detached along the greater part of its length from the wall of the gut, though it still remains connected with it, both at the venous and arterial ends.

Klein (Hien. Sitzungsbericht, LXIII. II., I871) considers that the heart is formed from the cells of the niesoblast of the splanchnopleure as a body which, at first solid, subsequently becomes hollow by the couversion of its central cells into blood-corpuscles. The layer of cells immediately surrounding the bloor-corpuscles forms the epithelial lining, and subsequently vecomes counected with that of the great arteries and veins.

The following view, which our own observations have led us to adopt, agrees with that of Klein in regarding the heart as being at first a solid thickening of the mesoblast of the splanchnopleure; but its accordance with the earlier statements of Von Baer is much more complete.

In order to understand the formation of the heart it uust be distinctly borne in mind that in the region where the heart is about to appear, the splanchnopleure is continually being folded in on either side, and that these lateral folds are progressively meeting and uniting in the middle line to form the under-wall of the foregut (that which in the adult chick $w$ ill be the anterior wall of some portion of the alimentary canal). (Compare Chap. II. $\$$.) At any given moment these folds will be found to have completely united in the middle line along a certain distance measured from the point in front where the cleavage of the mesublast (i.e. the separation into sumatopleure and splanchnopleure) begins, to a paiticular point tarther back.

At this particular point the folus will have met, but not united, Fig. 17, $A$. Further back still they will have not even met, but will appear simply inclined towards each other, Fig. $5, B$. Or, to put it in another way, they will here be found to be diverging from the point where they were united, and not only diverging laterally each from the niddle line, but also both turning so as to run in a forward direction to regain the surface of the yolk and rejoin the somatopleure, Fig. 16. In a transverse section taken behind this extreme point of union, or point of divergence, as we may call it, the splanchnopleure on either side when traced downwards from the axis of the embryo may be seen to bend in towards the middle so as to apprwach its fellow, and then to run rapidly outwards, Fig. I 7, $B$. A longitudinal section shews that it runs forwards also at the same time, Fig. 16. A section through the very point of divergence shews the two folds meeting in the middle line and then separating again, so as to form something like the letter $X$, with the upper limbs converging, and the lower limbs diverging. In a section taken in front of the point of divergence, Fig. I8, the lower diverging limbs of the $\mathrm{X}$ have disappeared altogether; nothing is left but the upper limbs, which, completely united in the middie line, form the under-wall of the toregut.

As development proceeds, what we have called the point of divergence is continually being carried farther and tarther back, so that the distince between it and the point where the somatopleure and splanchnopleure separate from each other in front, i.e. the length of the foregut, is continually increasing.

When the heart is about to bo formed, thickenings are observed in the mesoblast of the splanchnupleure, along the diverging folds, i.e. along the lower limbs of the $\mathbf{X}$ just behind the point of divergence. 'I hese thickenings are continued into each other by a similar thickening of the mesoblast extending through the point of divergence itself.

At first there is no thickening of the mesollast in front of the point of divergence, $i . \epsilon$. along the under-wall of the foregut. As the point of diverg- 
ence however is in the course of events carried farther hack, though the lower diverging folds (the lower limbs of the $\mathrm{X}$ ) risappear, the thickening at the point renıains and increases. In a short time, cousequently, we do find a thickening of the mesoblast in the under-wall of the foregut just in front of the point of divergence, which thickening is continuous like an inverted $X$, with two thickenings reaching down the diverging folds behind the point of divergence.

This $X$-shaped thickening becomes hollow by a transformation of its central cells; the single cavity in front is the cavity of the heart, and the two diverging cavities behind, with which it is continuous, are the canals of the omphalo-mesaraic veins.

As development proceeds, and the point of divergence is carried still farther and farther back, the heart increases in length step by step at the expense of the continually coalescing omphalo-mesaraic veins.

The coalescence of the mesoblastic thickening which forms the walls of the veins precedes that of their canals, consequently in sections taken at particular points we meet with two cavities invested by one wall. This is probably what was seen by the observers who have described the heart as being formed as a double tube which afterwards became single.

The front end of the cavity of the heart is continuous with canals similarly formed in the mesoblast of the foregut by the solution of certain cells. These are the canals of the aortæ.

At first the substance of the heart is along its whole length adherent to and indeed a part of the underwall of the foregut. Sub-equently it becomes free in its middle portion, the arterial and venous ends alone remaining attached.

Soon after its formation the heart begins to beat, its at first slow and rare pulsations beginning at the venous and passing on to the arterial end. It is of some interest to note that its functional activity commences long before the cells of which it is composed shew any distinct differentiation into muscular or nervous elements.

6. To provide channels for the fluid thus pressed by the contractions of the heart, a system of tubes has made its appearance in the mesoblast both of the embryo itself and of the vascular and pellucid areas. In front the single tube of the heart bifurcates into two primitive aorte, each of which bending round the front end of the foregut, passes from its under to its upper side, the two forming together a sort of incomplete arterial collar imbedded in the mesoblast of the gut. Arrived at the upper side of the gut, they turn sharply round, and run separate but parallel to each other backwards towards the tail, in the mesoblast on each side of the notochord immediately under the protovertebræ (Figs. 18, Ao, 20, Ao). About half way to the hinder extremity each gives off at right angles to the axis of the embryo a large branch, the omphalomesaraic artery (Fig. 23, Of, A.), which, passing outwards, is 
distributed over the pellucid and vascular areas, the main trunk of each aorta passing on with greatly diminished calibre towards the tail, in which it becomes lost.

FIG. 17. $A$.

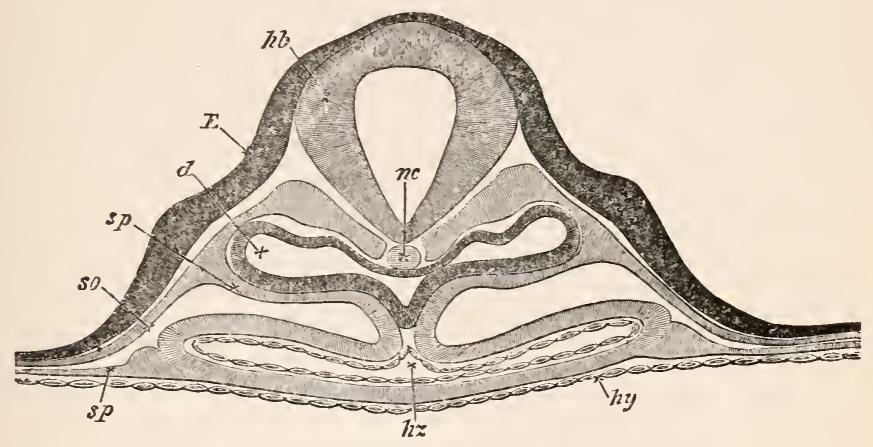

FIG. I7. $B$.

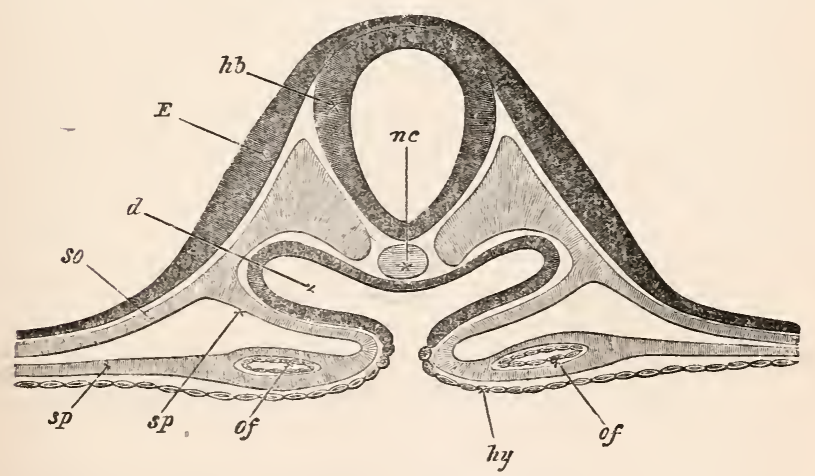

Two consecetive Sections of A 36 hours' Eybryo illdstrating the FORMATION OF THE HEIRT. $A$ IS THE MOST ANTERIOR OF THE TWO.

h. b. hind brain. nc. notochord. $E$. epiblast. so. somatopleure. sp. splanchnopleure. $d$. alimentary canal. hy. hypoblast. $h z$. (in $d$ ) heart. of. omphalo-mesaraic vein.

The heart is seen from the sections to be formed from the mesoblast of the splanchnopleure. It is not bowever split off from a portion of the mesoblast which forms the muscular wall of the alimentary canal, but the mesoblast, where it turns round to run outwards again over the yolk-sack, becomes thickened, and in each of the thickenings (one on each side) so formed, a cavity appears 
forming immediately behind the heart the omphalo-mesaraic veins (section $B$ ), (of). As however the folding of the splanchnopleure becomes more complete, and the digestive canal becomes completely closed (instead of remaining partially open as in section $B$ ), these two cavities unite; and an appearance is produced similar to that represented in figure $A$, where there is the single cavity of the heart $(h z)$. In the interior of the heart is seen a lining of flattened cells.

The shading, as will be seen, is purely diagrammatic. The epiblast, whether superficial as at $E$, or involuted as part of the neural canal $h b$, is shaded of one tiut. The mesoblast, whether uncleft, or diverging into somatopleure and splanchnopleure, is of another tint. In the hypoblast a distinction has been drawn between the thickened portion which lines the alimentary canal, and the thinner portion which belongs to the more peripheral part of the splanchnopleure, the two being at first continuous as in $B$, and afterwards separated as in $A$.

It will be understond that the two figures, though actually two consecutive sections of the same embryo, may be taken to represent two phases of the formation of the heart. $B$ in the process of development will become $A$, and $A$ a short time previously was in the condition of $B$.

Fig. I 8 .

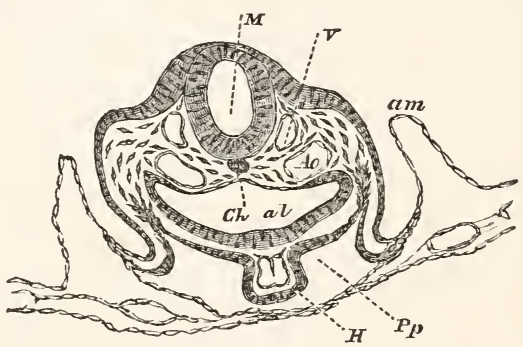

Transverse Section of an Embrto at the end of the second day passing THROUGH THE REgion of bULbUS arteriosus. (Copied from His.)

M. medullary canal in the region of the hind brain. $V$. anterior cardinal or superior vertebral vein. Ao. Aorta. Ch. Notochord. al. alimentary canal. $H$. Heart (bulbus arteriosus). Pp. Pleuroperitoneal cavity. am. amnion.

On comparing this with Fig. 17 , it will be seen that the mesoblast (muscular) wall of the heart $I I$ has now become quite separate from the rest of the mesoblast of the splanchnopleure, which forms, in the section, an independent line below the heart, the section of branches of the omphalo-mesaraic veins being seen on either side. The bridle of mesoblast represented in the drawing as passing from the splanchnopleure below to the somatopleure above, reaching the latter just inside the fold of the annion, has been described by His, but has never been seen by ourselves.

In the vascular and pellucid areas, the formation of vascular channels with a subseqnent differentiation into arteries, capillaries and veins, is proceeding rapidly. Blood- 
corpuscles too are being formed in considerable numbers. 'The mottled yellow vascular area becomes covered with red patches consisting of aggregations of blood-corpuscles, often spoken of as blood-islands.

Round the extreme margin of the vascular area and nearly completely encircling it, is seen a thin red line, the sinus or vena terminalis (Fig. 23, $S v$.). This will soon increase in size and importance.

From the vascular and pellucid area several large channels are seen to unite and form two large trunks, one on either side, which running along the splanchnopleure folds at nearly right angles to the axis of the embryo, unite at the "point of divergence" to join the venous end of the heart. These are the omphalo-mesaraic veins (Figs. 14, o.f., 23, o.f.) spoken of above.

Both vessels and corpuscles are formed entirely from the cells of the mesoblast; and in the regions where the mesoblast is cleft, are at first observed exclusively in the splanchnopleure. Ultimately of course they are found in the mesoblast everywhere.

The mode of formation of the blood-vessels and corpuscles has been much and long debated. The observations of one of us have led us to believe the following to be the true account.

In the pellucid area, where the formation of blood-vessels may be most easily observed, a number of mesoblastic cells are seen to send out processes. These processes unite, and by their union a protoplasmic network is formed containing nuclei at the points from which the processes started. The nuclei, which as a rule are much elongated and contain large oval nucleoli, increase very rapidly by division, and thus form groups of nuclei at the, so to speak, nodal points of the network. Several nuclei may also be seen here and there in the processes themselves. The network being completed, these groups, by continued division of the nuclei, increase rapidly in size; the majority of the nuclei composing them acquire a red colour and become converted into blood-corpuscles (Fig. I9, b.c.); but a few, generally on the outside of the group, remain ununaltered, (Fig. I 9, a). The protoplasm in which the central reddened nuclei are imbedded becomes liquefied, while that on the outside of each group, as well as that of the uniting processes, remains granular, and increasing in quantity, forms an investment for the unaltered nuclei which are embedded in it.

Each nodal point is thus transformed into a more or less rounded mass of blood-corpuscles floating in plasma but enveloped by a layer of nucleated protoplasm, the several groups being united by strands of nucleated protoplasm. These uniting strands rapidly increase in thickness; new processes are also continually being formed; and thus the network is kept close and thickset while the area is increasing in size.

By a transformation of nuclei similar to that which took place in the nodal points, blood-corpuscles make their appearance in the processes also, the central portions of which become at the same time liquefied. The uncoloured nuclei 
situate in the envelopes of the nodal groups, as well as those lying on the exterior of the connecting processes, appropriate a quantity of the granular protoplasm surrounding each, and thus become converted into spindle-shaped cells. Each nodal group and each connecting process thus gets a distinct wall of nucleated cells. By the continued widening of the connecting processes and solution of their central portions, accompanied by a corresponding increase in the enveloping nucleated cells, the original protoplasmic network is converted into a system of communicating tubes, the canals of which contain blood-corpuscles and plasma, and the walls of which are formed of spindle-shaped nucleated cells.

FIg. 19.

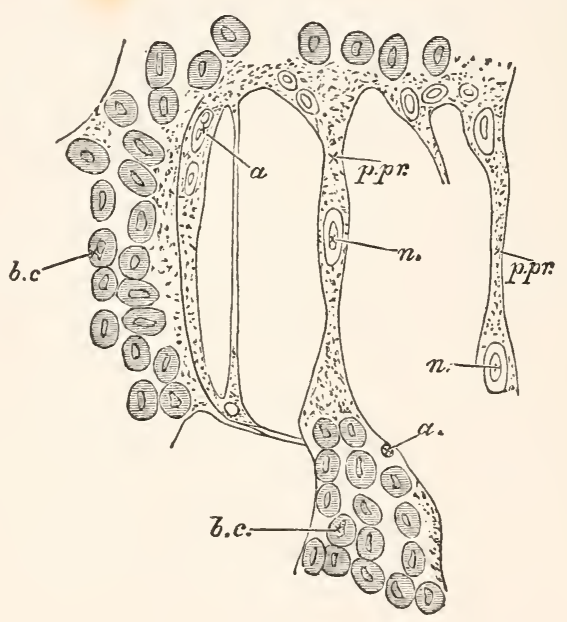

SURFACE VIEW FROM BELOW OF A SMALL PORTION OF THE POSTERIOR END OF THE PELLUCID AREA OF A 36 HOURS' CHICK. 'To illustrate the formation of the blood-capillaries and blood-corpuscles, magnified 400 diameters.

b. c. Blood-corpuscles at a nodal point, already beginning to acquire a red colour. They are enclosed in masses of protoplasm in the outermost layer of which are found nuclei, $a$, some of which contain two nucleoli. These nuclei subsequently become the nuclei of the cells forming the walls of the vessels. The nodal groups are united by protoplasmic processes $(p \cdot p r)$, also containing nuclei with large nucleoli $(n)$. These nuclei increase in number by division, and become converted in part into the nuclei of the cells forming the walls of the vessels, and in part into blood-corpuscles.

The blood-corpuscles pass freely from the nodal points into the hollow processes, and thus the network of protoplasm becomes a network of blood-vessels ; the corpuscles and the nuclei of the walls of which have been by separate paths of developinent derived from the nuclei of the original protoplasm.

The formation of the corpuscles does not proceed equally rapidly or to the 
same extent in all parts of the blastorlerm. By far the greater part are formed in the vascular area, but some arise in the pellucid area, especially in the hinder part. In the front of the pellucid area the processes are longer and the network accordingly more open; the corpuscles also are both later in appearing and less numerous when formed.

The omphalo-mesaraic arteries and veins, and the sinus terminalis which from the first has a distinct wall, seem to take origin in a manner altogether similar to that of the smaller vessels; and the description of the formation of the heart which we gave above shews that it too is nothing but a gigantic nodal point.

Assuming the truth of the above account, it is evident that the blood-vessels of the chick do not arise as spaces or clsannels between adjacent cells of the mesoblast, but are hollowed out in the communicating protoplasmic substance of the cells themselves. It is also perhaps worthy of note that the red-blood corpuscles are not cells, but nuclei.

The red-blood corpuscles when removed from the vessels exhibit energetic amœboid movements. They seem to increase at this stage chiefly by division.

The above is the view which we deduce from our own observations. The following may serve as a brief summary of the history of the matter.

Von Baer and the older embryoloyists regarded the blood-vessels as being at first mere gaps or spaces between the cellular elements of the mesoblast, hollowed out so to speak by the flow of blood from the heart. The first steps in the right direstion were taken by Remak and Kölliker, who described the formation of solıd bands or cylinders composed of cells and arranged in a close-set network. These bands, becoming hollowed by solution while their central cells were converted into blood-corpuscles, gradually put on the appearance of blood-vessels, the aggregation of the red corpuscles at varinus points, through arrest of the circulation, giving rise to the blood-islands of Wolft and Pander.

According to Afanassieff (Wien. Sitz. Bericht. Bd. 53, I 866) there appear in the mesublast vesicles of variable size, with protoplasmic envelopes and contents. These vesicles, which are at first clear and homogeneous, subsequently become traversed with strands of nucleated protoplasin, forming often a close net-work within the vesicle. The space intervening between the numerous vesicles is cut up into a network of canals by protoplasmic processes stretching from one vesicle to another. These canals are the rudimentary blood-vesse!s. From the outside of the vesicles, forming the inner wall of the adjacent vessels, nucleated masses of protoplasm are budded off as blood-corpuscles and fall into the current of the circulation.

His (op. cit.), following out his peculiar theory of development, derived both blond and blood-vessels from the white yolk or parablast. According to him while certain of the white-yolk masses become converted into conglomerations of cells, which acquiring a yellow colour stand out in surface views as bloodislands, other white-yolk masses, metamorphosed into angular cells, form a network of thick lines permeating the mass of true blastodermic (archiblastic) cells of the mesoblast. These lines, at the first solid, subsequently become hollow. The meshwork of canals, or rudimentary blood-vessels, thus developed first in the vascular and pellucid areas and spreading thence into the embryo, contains for a certain time clear fluid only, the blood-islands being imbedded in or attached to the walls of the canal and surrounced by protoplasmic envelopes, so that the blood-corpuscles are shut out from the cavities of the ressels. Later on, however, the envelopes of the islands are broken through, and the blood-corpuscles emerging from their nests fall into the current of the circulation.

His therefore regarded the blood-corpuscles as formed in greater part at least 
separate'y from the blood-vessels; their entrance into the vascular spaces being an after event. The parablastic cells (derived from the white yolk), in his view, give rise to the epithelium (endothelium) and connective tissue elements only of the blond vessels, the muscular elements being derived from genuine (blasto. dermic) mesoblastic cells.

Klein (Wien. Sitz. Bericht. LxII. I87I) describes the blood-vessels as taking their origin from certain cells of the mesoblast in which a vacuole, appearing and rapidly increasing in size, pushes the nucleus on one side, leaving only a thin layer of protoplasm round the periphery of the cell. In this thin layer nuclei appear; and, multıplying, form a complete nucleated investment. to the vacuole, which nean while continues to increase in size. From the inside of this protoplasmic investment cells are budded off, and fall into the vacuole. Here they soon acquire a red colour and become converted into blood-corpuscles. From the exterior of these vacuolated cells nucleated processes are thrown out, which end freely or join with similar processes from other cells. A protoplasmic network is thus forned, the lines of which become vacuolated, and hollow, and ultimately comnunicate with the original central vacuoles now crowded with corpuscles. By these means a system of communicating tubes is established. Klein also describes two other forms of cells somewhat differing from the above, but also taking part in the formation of the blood-vessels. One of these forms is found chiefly in the vascular area, and he believes that these latter are sinuply the formative cells of which we have alrearly so often spoken.

It will thus be seen that Klein's view, from which our own differs chiefly in reference to the matter of vacuolation, is a return, with some nodifications and extensions, to the earlier view of Remak, and that the accounts of both Afanassieff and His, which in turn agree in miny respects, have proved to be uncorroborated divergences from the older track.

Suil more recently Goette (Archiv für Micro. Anat. Vol. x. 1873, pp. 145-199) has given an entirely different account of the origin of the bloodvessels and bloor-corpuseles in the vascular area. He believes that in the thick mass of cells immediately outside the 'pellucid area' (vide Chap. III. $\$ 12$ ) a quantity of fluid collects and causes the cells to separate into a new work with large spaces fillerl with fluid. Into these spaces the formative cells travel, and undergoing a species of endogenous cell-formation, form masses of bl odcorpuscles - the blood-islands of the earier authors. This view differs, it will be seen, from all the later views, and goes back to that of Von Baer in regarding the blood-ıessels as primitively mere gaps between the cellular elements. In the investigation of such a point as this, sections (which apparently Goette has alone employed) are very untrustworthy.

7. The cells of the epiblast and hypoblast as well as of the mesoblast undergo considerable changes between the $24^{\text {th }}$ and the $3^{6}$ th hour.

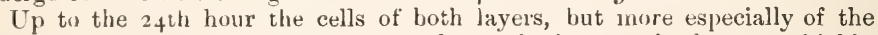
hypoblast, were filled with fine granules and also contained many highly refractive spherules. By the $3^{6 \text { th }}$ hwur, however, they have become nuch more transparent. Each cell now consists of a clear protoplasm with hardly any granules or spherules, and a large oval nucleus together with one or more vacuoles is distincaly visible.

The cells of the hypublast still pass insensibly into the white-yolk cells; and it is still by the conversion of the white yolk into hypoblast that the peripheral extension of the latter is chiefly carried sn.

'The bypoblast cells beneath and at the sides of the embryo are markedly smaller than those at the periphery of the pellueid area.

The epiblast cells exhibit considerable variation in size in different parts of 
the embryo; but all are considerably smaller and also somewhat more columnar than the more peripher.s cells of the pellucid area. The largest epiblastic cells are to be found in the region of the vaseular area, but here they are much flattened. At the extreme outrr edge of the opaque area the cells are smaller again, shewing in this respect a marked contrast to their condition during the previous stage.

8. About this period there may be seen in transverse sections, taken through the embryo in the region of the protovertebræ, a small group of cells (Fig. 20, $W . d$ ) projecting on either side from the mass of uncleft mesoblast on the outside of the protovertebræ, into the somewhat triangular space formed by the epiblast above, the upper and outer angle of the protovertebra on the inside, and the mesoblast on the outside.

This gromp of cells is the section of a longitudinal ridge, the rudiment of the Wolffian duct. We shall return to it immediately.

9. The most important changes then which take place during the first half of the second day, are the closure of the medullary folds, especially in the anterior part, and the dilatation of the canal so formed into the first cerebral vesicle ; the establishment of a certain number of protovertebræ; the elevation of the head from the plane of the blastoderm; the formation of the tubular heart and of the great blood-vessels; and the appearance of the rudiment of the Wolffian duct. It is important to remember that the embryo of which we are now speaking is simply a part of the whole germinal membrane, which is gradually spreading over the surface of the yolk. It is important also to bear in mind that all that part of the embryo which is in front of the most anterior protovertebræ corresponds to the future head, and the rest to the neck, body and tail. At this period the head occupies nearly a third of the whole length of the embryo.

\section{The changes which take place from the 36 th to} the 45th hour will best form the next stage, since those which occur during the last few hours of the second day will be more conveniently described with the third day.

One important feature of the stage is the rapid increase in the process of the folding off of the embryo from the plane of the germ, and its consequent conversion into a distinct tubular cavity. At the beginning of the day, the head alone projected from the rest of the germ, the remainder of the 
FIG. 20.
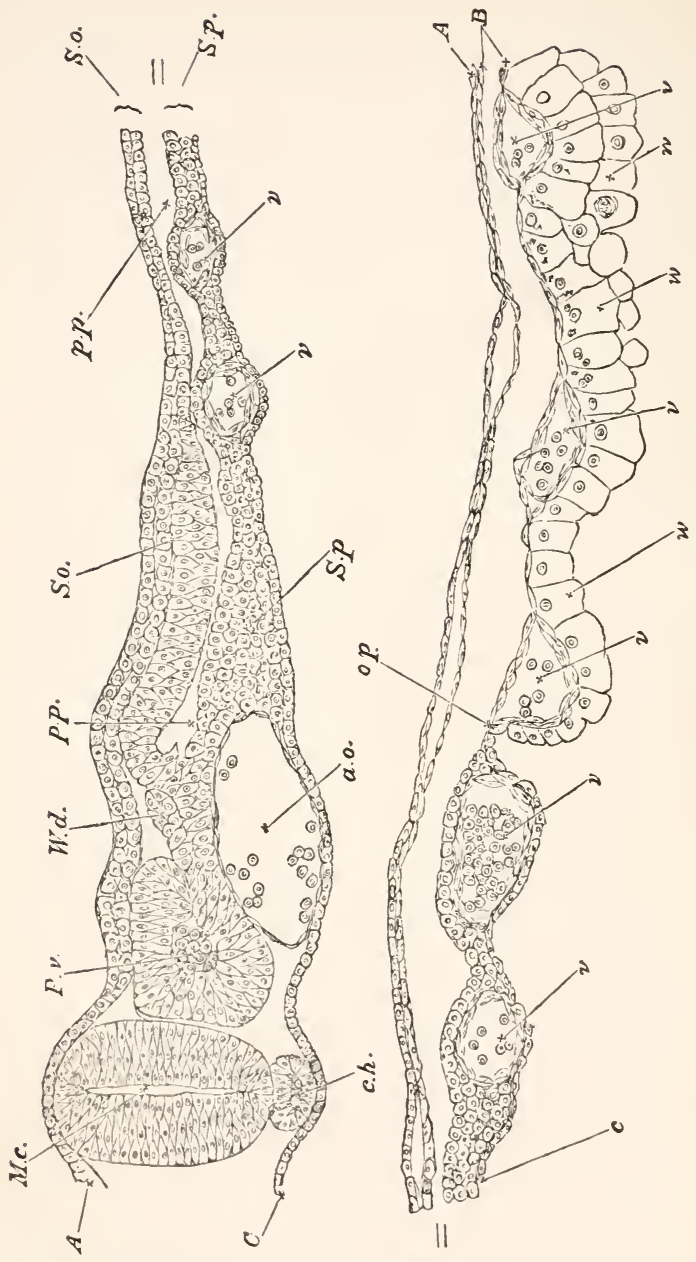

Transverse Section through the Dorsal Region of an Embrio of 45 HOURS.

A. epiblast. B. mesoblast. C. hypoblast consisting of a single row of flattened cells. M. c. medullary canal. P. v. protovertebra. W. $d$. Wolffian duct. S. o. Somatopleure. S. $p$. Splanchnopleure. $p \cdot p$. pleuroperitoneal 
cavity. c. h. notochord. a.o. dorsal aorta. $v$. blood-vessels of the yolk-sac. o. $p$. line of junction between opaque and pellucid areas; at this point the hypoblast cells are seen to pass without any strong line of demarcation into the white-yolk spheres. $w$. white-yolk spheres, some of which near the edge of the pellucid area contain a body very like a nucleus.

Only one half of the section is represented in the figure-if completed it would be bilaterally symmetrical about the line of the medullary caual.

FIG. 2 I.

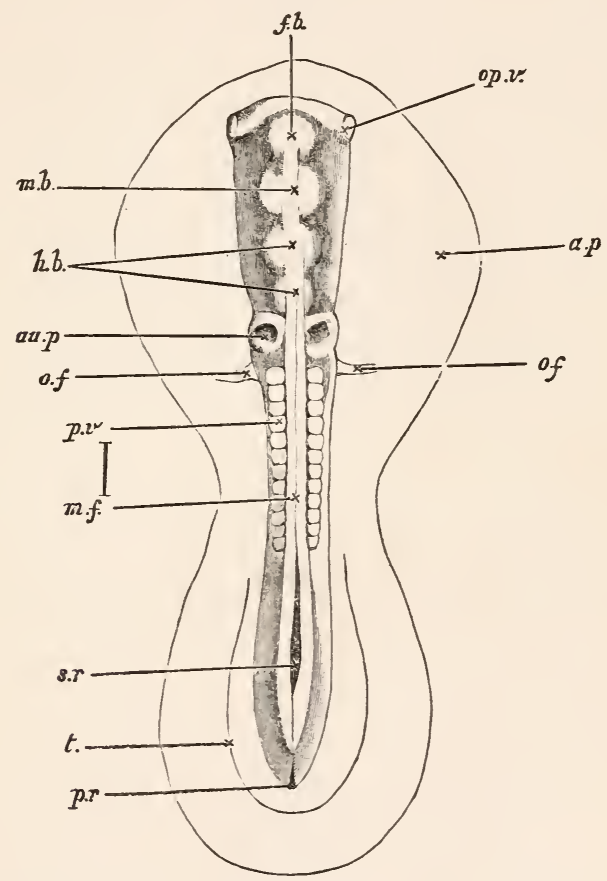

Embryo of the Chick at 36 hours viewed from above as an OPaque Овлест. (Chromic acid preparation.)

$f . b$. front brain. $m . b$. mid brain. h. b. hind brain. op. v. optic vesicle. $a u . p$. auditory vesicle. o. $f$. omphalo-mesaraic vein. $p . v$. protovertebra. $m$. $f$. line of junction of the medullary folds above the medullary canal. $s . r$. sinus rhomboidalis. $t$. tail-fold. $p$. $r$. remains of primitive groove. a. $p$, area pellucida.

embryo being simply a part of a flat blastoderm, nearly completely level from the front protovertebræ to the hind edge of the pellucid area. At this epoch, however, a tail-fold 
(Fig. 21, $t$ ) makes its appearance, elevating the tail above the level of the blastoderm in the same way that the head was elevated. Lateral folds also, one on either side, soon begin to be very obvious. By the progress of these, together with the rapid backward extension of the head-fold and the slower forward extension of the tail-fold, the body of the embryo becomes more and more distinctly raised up and marked off from the rest of the blastoderm.

11. 'The medullary canal closes up rapidly. The wide sinus rhomboidalis becomes a narrow fusiform space (Fig. 21, s.r.), and at the end of this period is entirely roofed over. The conversion of the original medullary groove into a closed tube is thus completed.

12. In the region of the head most important changes now take place. We saw that at the beginning of this day the front end of the medullary canal was dilated into a buib, the first cerebral vesicle. This, from the very first broader than long, now increases so much in breadth as to give the embryo a hammer-headed appearance. The lateral portions. continuing to enlarge, become after a while separated by constrictions from the central portion. The single vesicle is thus converted into three vesicles: a median one connected by short hollow stalks with a lateral one on either side. The lateral vesicles are known as the optic vesicles (Fig. 21, op. $v$, Fig. 22, a), and will afterwards become converted into parts

FIG. 22.

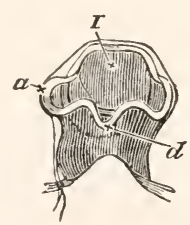

Head of a Chick at the End of the Second Day viewed from below as a Transparent Object. (Copied from Huxley).

I. first cerebral vesicle. $\boldsymbol{c}$ optic vesicle. $d$. infundibulum.

The specimen shews the formation of the optic vesicles $(a)$, as outgrowths from the Ist cerebral vesicle or vesicle of the 3 rd ventricle, so that the optic vesicles and vesicle of the 3 rd ventricle at first freely communicated with each other, and also the growth of the lower wall of the vesicle of the 3 rd ventricle into a process which becomes the infundibulum $(d)$. 
of the eyes; the median one still retains the name of the first cerebral vesicle. The constriction takes place chiefly from above downwards, so that the optic vesicles soon appear to spring from the under portions of the cerebral vesicle.

The original vesicle being primarily an involution of the epiblast, the walls of all three vesicles are formed of epiblast; all three vesicles are likewise covered over with the common epiblastic investment which will eventually become the epidermis of the skin of the head. Between this superficial epiblast and the involuted epiblast of the vesicles, there exists a certain quantity of mesoblast to serve as the material out of which will be formed the dermis of the scalp, the skull, and other parts of the head. At this epoch, however, the mesoblast is found chiefly underneath the several vesicles. A small quantity may in section be seen at the sides; but at the top the epidermic epiblast is either in close contact with the involuted epiblast of the cerebral and optic vesicles or separated from it by fluid alone, there being as yet in this region no cellular elements between the two representing the mesoblast.

The constrictions marking off the optic vesicles take place of course beneath the cummon epiblastic investment, which is not involved in them. As a consequence, though easily seen in the transparent fresh embryo (Fig, 22), they are but slightly indicated in hardened specimens (Fig. 21). In sections they are very clearly seen.

13. When an embryo of the early part of the second day is examined as a transparent object, that portion of the medullary canal which lies immediately behind the first cerebral vesicle is seen to be conical in shape, with its walls thrown into a number of wrinkles. These wrinkles may vary a good deal in appearance, and shift from time to time, but eventually, before the close of the second day, after the formation of the optical vesicles, settle down into two constrictions, one separating the first cerebral vesicle from that part of the medullary canal which is immediately behind it, and the other separating that second portion from a third. So, instead of there being one cerebral vesicle only, as at the commencement of the second day, there is now, in addition to the optic vesicles, a series of three, one behind the other; a second and third cerabral vesicle have been added to the 
first (Fig. 21, $m b, h b$ ). They may be also called the "fore brain," the "mid brain," and the "hind brain," for into these parts will they eventually be developed.

14. The optic vesicles, lying underneath the epiblast, towards the end of the day are turned back and pressed somewhat backwards and downwards against the sides of the first cerebral vesicle or fore brain, an elongation of their stalks permitting this movement to take place. The whole head becomes in consequence somewhat thicker and rounder.

15. Before the end of the day the fore brain, by a process similar to that whereby the optic vesicles were formed, viz. undue growth followed by constriction, has begun to bud off two small vesicles in front; these are the vesicles of the cerebral hemisplueres, which subsequently become the most conspicuous part of the brain, but up to the end of the day are still very small and inconspicuous.

16. The notochord, whose origin was described in the account of the first day (Chap. III. $\S \check{5}$ ), is during the whole of the second day a very conspicuous object. It is seen as a transparent rod, somewhat elliptical in section (see Fig. 20, ch), lying immediately underneath the medullary canal for the greater part of its length, and reaching forward in front as far as below the centre of the second cerebral vesicle, where it ends either in a point (Remak), or in a rounded knob (Baer, Dursy, Entwickelungsgeschichte des Kopfes). The exact relations of its termination will be discussed later on.

Round the anterior termination of the notochord, the medullary canal, which up to the present time has remained perfectly straight, towards the end of the day begins to curve. The front portion of the canal, i.e. the fore-brain with its optic and cerebral vesicles, becomes slightly bent downwards, so as to form a rounded obtuse angle with the rest of the embryo. This is the commencement of the so-called cranial flexure.

17. Lastly, as far as the head is concerned, the rudiment of the ear appears about this time on the dorsal surface as a small depression or pitting of the epiblast on either side of the hind-brain (Fig. 21, au. p).

1S. We left the heart as a fusiform body slightly bent to the right, attached to the under wall of the foregut by the 
aorta and by its venous end, but with its intermediate portion quite free. The curvature now increases so much that the heart becomes almost $\Omega$-shaped, the venous portion being drawn up towards the head so as to lie somewhat above (dorsal to) and behind the arterial portion. (It would perhaps be more correct to say that the free intermediate portion is by its own growth bent downwards, backwards, and somewhat to the right, while the venous root of the heart is at the same time continually being lengthened by the carrying back of that "point of divergence" of the splanchnopleure folds which marks the union of the omphalo-mesaraic veins into a single venous trunk). The heart then has at this time two bends, the one, the venous bend, the right-hand curve of the $\boldsymbol{\Omega}$; the other, the arterial bend, the left-hand curve of the $\boldsymbol{\Omega}$. The venous bend which, as we have said, is placed above and somewhat behind the arterial bend, becomes marked by two bulgings, one on either side. These are the rudiments of the auricles, or rather of the auricular appendages. The ascending limb of the arterial bend soon becomes conspicuous as the bulbus arteriosus, while the rounded point of the bend itself will hereafter grow into the ventricles.

19. The blood-vessels, whose origin during the first half of this day has been already described, become during the latter part of the day so connected as to form a complete system, through which a definite circulation of the blood is now for the first time (consequently some little while after the commencement of the heart's pulsation) carried on.

The two primitive aortce liave already been described as encircling the foregut, and then passing along the body of the embryo immediately beneath the protovertebræ on either side of the notochord. They are shewn in Fig. 20 a.o in section as two large rounded spaces lined with spindle-shaped cells. At first they run as two distinct canals along the whole length of the embryo; but, after a short time, unite at some little distance behind the head into a single trunk, which lies in the middle line of the body immediately below the notochord (Fig. 39). Lower down, nearer the tail, this single primitive trunk again divides into two aortæ, which, getting smaller and smaller, are finally lost in the small blood-vessels of the tail. At this epoch, therefore, there are two dortic arches springing from the bulbus arteriosus, and 
uniting above the alimentary canal in the back of the embryo to form the single dorsal aorta, which travelling backwards in the median line divides near the tail into two main branches. From each of the two primitive aortw, or from each of the two branches into which the single aorta divides, there is given off on either sicle a large branch. These have been already spoken of as the omplato-mesaraic arteries. At this stage they are so large that by far the greater part of the blood passing lown the aorta finds its way into them, and a small remnant only pursues a straight course into the continuations of the aorta towards the tail

Each omphalo-mesaraic artery leaving the aorta at nearly right angles (at a point some little way behind the backward linit of the splanchnopleure fold which is forming the alimentary canal), runs outwards beneath the protovertebre in the lower range of the mesoblast, close to the hypoblast. Consequently, when in its course outwards it reaches the point where the mesoblast is cleft to form the somatopleure and splanchnopleure, it attaches itself to the latter. 'Travelling along this, and dividing rapidly into branches, it reaches the vascular area in whose network of small vessels (and also to a certain extent in the similar small vessels of the pellucid area) it finally loses itself.

The terminations of the omphalo-mesaraic arteries in the vascular and pellucid areas are further connected with the heart in two different ways. From the network of capillaries, as we may call them, a number of veins take their origin, and finally unite into two main trunks, the omphalo-mesaraic veins. 'These have alrearly been described as running along the folds of the splanchnopleure to form the venous roots of the heart. Their course is consequently more or less parallel to that of the omphalo-mesaraic arteries, but at some little distance nearer the head, inasmuch as the arteries run in that part of the splanchnopleure which has not yet been folded in to form the alimentary canal. Besides forming the direct roots of the omphalo-mesaraic veins, the terminations of the omphalo-mesaraic arteries in the vascular area are also connected with the sinus terminalis spoken of above as running almost completely round, and forming the outer margin of the vascular area. This (Fig. $23, s . v$ ), may be best described as composed of two semicircular canals, which nearly 
meet at points opposite the head and opposite the tail, thus all but encircling the vascular area between them. At the point opposite the head the end of each semicircle is connected with vessels (Fig. 23), which run straight in towards the heart along the fold of the splanchnopleure, and join the right and left omphalo-mesaraic veins. At the point opposite the tail there is at this stage no such definite connection. At the two sides, midway between their head and tail ends, the two semicircles are especially connected with the omphalo-mesaraic arteries.

The circulation of the blood then during the latter half of the second day may be described as follows. The blood brought by the omphalo-mesaraic veins falls into the twisted cavity of the heart, and is driven thence through the bulbus arteriosus and aortic arches into the aortic trunk. From the aorta, by far the greater part of the blood flows into the omphalo-mesaraic arteries, only a small remnant passing on into the caudal terminations. From the capillary net-work of the vascular and pellucid area into which the omphalomesaraic arteries discharge their contents, part of the blood is gathered up at once into the lateral or direct trunks of the omphalo-mesaraic veins. Part however goes into the middle region of each lateral half of the sinus terminalis, and there divides on each side into two streams. One stream, and that the larger one, flows in a forward direction until it reaches the point opposite the head, thence it returns by the veins spoken of above, straight to the omphalo-mesaraic trunks. The other stream flows backward, and becomes lost at the point opposite to the tail. This is the condition of things during the second day; it becomes considerably changed on the succeeding day.

At the time that the heart first begins to beat the capillary system of the vascular and pellucid areas is not yet completed; and the fluid which is at first driven by the heart contains, according to most observers, very few corpuscles.

20. At the close of the second day the single pair of aortic arches into which the bulbus arteriosus divides is found to be accompanied by a second pair, formed in the same way as the first, and occupyng a position a little behind it. Sometimes even a third pair is added. Of these aortic arches we shall have to speak more fully later on. 
21. At the latter end of this day, the ridge which we have already spoken of as the rudiment of the Wolffian duct, has become distinctly hollow, is in fact no longer a ridge but a canal. Sections now shew not an irregular group of ordinary mesoblastic cells, but a small cavity surrounded by a wall of cells; and these cells are beginning to put on a columnar character, and thus appear to radiate from the central carity. 'The canal or duct so formed, the anterior termination of which is closed, and the posterior not as yet completely formed, reaches from about the fifth pair if protovertebre backwards towards the hind end of the embryo. The conversion of the ridge into a canal appears to take place by the cells acquiring a radiating arrangement, and a small hole appearing at the centre where the points of the cells meet; this rapilly grows larger till it reaches the final size of the cavity of the duct.

The exact mode of development of the Wolffian duct is still a matter of some doubt, although its origin has been investigated by numerous embryologists.

lemak, and after him Kölliker, described it as taking its origin from the mesoblast of the somatopleure, and appearing about the middle of the second day, at the external border of the protovertebre immediately under the epiblast, in the form of a solid cord which subsequently became hollow.

Dursy (Zcitsch. f. Ret. Med. I86;) gave a very similar account, except that he regarded it as being derived from the substance of the protovertebre, instead from the somatopleure.

Hensen (Arcliv. Microscop. Anat. Bd. III. I867), and for some time His, believed that the duct took origin as a longitudinal involution of the epillast between the protovertebræ and the lateral mesoblast, in the form of a groove, which subsequently becanse closed in and detached from the superficial epiblast, in a manner very similar to the way in which the lens is formed.

Subsequently His to k up the view that it was a product of the proto. vertebre, the central cells of these bodies, according to hine, protruding as a ridge along their upper and external angles. He states that at first a distinct connection is visible between the Wolffian duct and the central cells of the protovertebræ.

Waldeyer (Eicrstock $u . E i$, 1870) has given a totally different account. Between the external border of the protovertebrre, and the point where the mesoblast splits into somatopleure and splanchnopleure, there lies a mass of cells, which we shall have occasion to speak of hereafter as the intermediate cellmass. According to Waldeyer, the upper surface of this mass grows up into a nutrow ridge, seen in sections as a tongue-shaped process projecting into the vacant space (i.e. the space filled with fluid only) which exists below the epiblast at this point. Later on, this tongue-shaped process is seen to curve outwards, and thus to become hook-shined ; and the point of the hook subsequent'y unites with a similar smaller process derived from the more external portions of the same cell-rnass. The small cavity thus seen to be enclosed by a larger and smaller process, is of course the sectional view of a canal enclosed by a larger and smaller ridge. This canal is the Wolffian duct. Waldeyer further bellieves that the cells which thus form the walls of the duct are primarily epi- 
blastic in origin, having been separated from the epiblast at the epoch of the apparent fusion of the epiblast and mesoblast in the region of the primitive streak or axis-cord of His. This view, prompted as it evidently is by theoretical considerations, must be regarded as untenable, since the primitive streak has nothing whatever to do with the permanent body of the embryo.

Quite recently Romiti (Archiv. Microscop. Anat. x. $18_{i+}$ ) has described the Wolffian duct as being formed by an involution of the epithelium of the pleuroperitoneal cavity, in the form of a longitudinal groove which is thrust up into the superior portions of the intermediate cell-mass, and the communication of which with the pleuroperitoneal cavity is speedily obliterated. Such a mode of origin recommends itself to the embryologist, inasmuch as it is certainly the way in which, as we shall see, the Wolffian duct is formed in Amphibia and Osseous Fishes. For that very reason however it should be received with caution; all the more since the sections drawn by Romiti, and described is supporting his views, evidently belong to a staye considerably later than that at which the duct first distinctly appears. We bope to be able to shew, in the second part of this work, that the mode of development of the Wolffian duct described above, and which we believe to be the real one, is not so abnormal as it might at first sight be supposed to be.

22. The amnion, especially the anterior or head-fold, advances in growth very rapidly during the second day, and at its close completely covers the head and neck of the embryo; so much so that it is necessary to tear or remove it when the head has to be examined in hardened opaque specimens. 'The tail and lateral folds of the anmion, though still progressing, lag considerably behind the heal-fold.

23. The chief events then which occur during the second half of the second day are as follows :-

1. The second and third cerebral vesicles make their appearance behind the first.

2. The optic vesicles spring as hollow buds from the lateral, and the vesicles of the cerebral hemispheres from the front portions of the first cerebral vesicle.

3. The first rudiment of the ear is formed as an involution of the epiblast on the side of the hind-brain or third cerebral vesicle.

4. The first indications of the cranial flexure become visible.

ร. The head-fold, and especially the splanchnopleur: moiety, advances rapidly backwards ; the head of the embry., is in consequence more definitely formed. The tail-fold also becomes distinct.

6. The curvature of the heart increases; the first rurliments of the auricles appear.

7. The circulation of the yolk-sac is completed.

S. The amnion grows rapidly. 


\section{CHAPTER $T$.}

THE CHANGES WHICH TAKE PLACE DURING THE THIRD DAY.

1. OF all days in the history of the chick within the egg this perhaps is the most eventful ; the rudiments of so many important organs now first make their appearance.

On opening an egg on the third day, the first thing which attracts notice is the diminution of the white of the egg. This seems to be one of the consequences of the functional activity of the newly-established vascular area whose bloodressels are engaged either in directly absorbing the white or, as is more probable, in absorbing the yolk, which is in turn replenished at the expense of the white. The absorption, once begun, goes on so actively that, by the end of the day, the decrease of the white is very striking.

2. The blastoderm has now spread over about half the yolk, the extreme margin of the opaque area reaching about inalf-way towarls the pole of the yolk opposite to the embryo.

'The vascular area, though still increasing, is much smaller than the total opaque area, being in average-sized eggs about as large as a florin. Still smaller than the vascular area is the pellucid area in the centre of which lies the rapidly growing embryo.

3. During the third day the vascular area is not only a means for providing the embryo with nourishment from the rolk, but also, inasmuch as by the diminution of the white it is brought close under the shell and therefore fully exposed to the influence of the atmosphere, serves as the chief organ of respiration.

'This in fact is the period at which the vascular area may he said to be in the stage of its most complete development; 
for though it will afterwards become larger it will at the same time become less definite and less important. We may therefore, before we proceed, add a few words to the description of it given in the last chapter.

FIG. 23.

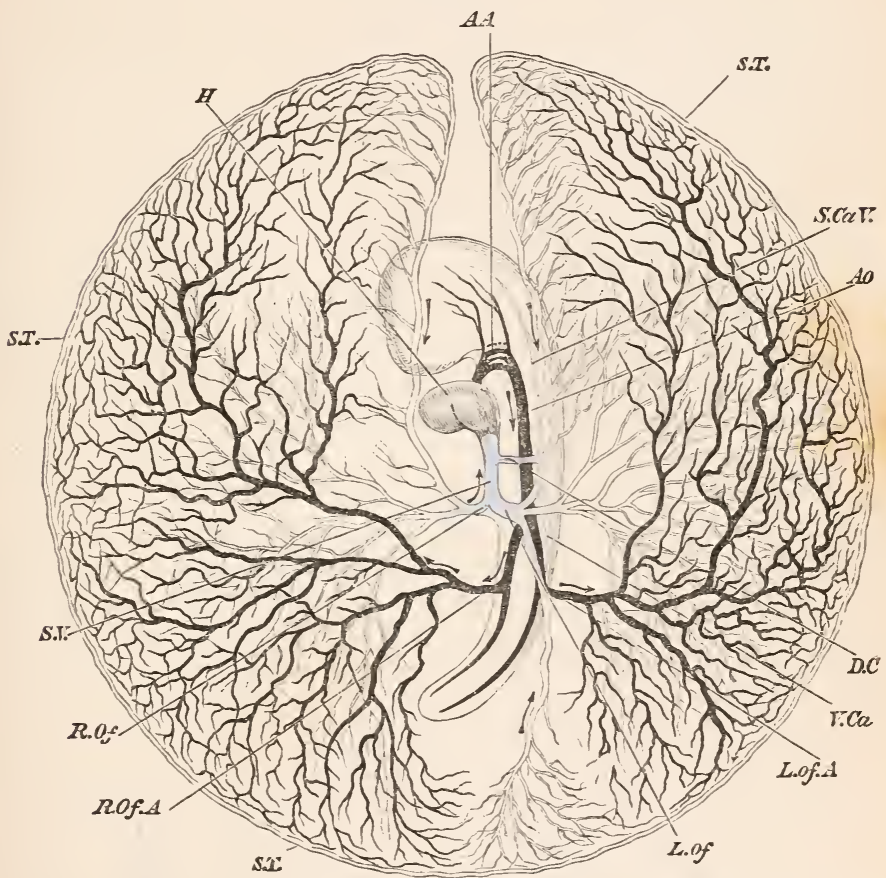

Diagray of the Circulation of the Yolk-Sack at the end of the ThiRd Day of Incubation.

$H$. heart. $A A$. the second, third and fourth aortic arches; the first has become obliterated in its median portion, but is continued at its proximal end as the external carntid, and at its distal end as the internal carotid. AO. dorsal aorta. $L . O f$. $A$. left omphalo-mesaraic artery. R.Of.A. right omphalo-mesaraic artery. S. T. sinus terminalis. L. Of. left omphalo-mesaraic vein. $R . O f$. right omphalo-mesaraic vein. $S . V$. sinus venosus. $D . C$. ductus Cuvieri. $S$. Ca. $\mathrm{V}$. superior cardinal vein. V.C $a$. inferior cardinal vein. The veins are marked in outline and the arteries are made black. The whole blastoderm has been removed from the egg and is supposed to be viewed from below. Hence the left is seen on the right, and vice versa. 
The blood leaving the body of the embryo by the omphalomesaraic arteries (Fig. 23, L. Of. A., L. Of. A.), is carried to the small vessels and capillaries of the vascular area, a small portion only being appropriated by the pellucid area.

From the vascular area part of the blood returns directly to the heart by the main lateral trunks of the omphalomesaraic veins, $R$. Of., L. Of. During the second day these venous trunks joined the body of the embryo considerably in front of, that is, nearer the head than, the corresponding arterial ones. Towards the end of the third day, owing to the continued lengthening of the heart, the veins and arteries run not only parallel to each other, but almost in the same line, the points at which they respectively join and leave the body being nearly at the same distance from the head. According to Von Baer and other observers the veins in the vascular area are placed nearer the surface than are the arteries. Close to the body the reverse is the case; near the body therefore they cross over each other.

The rest of the blood brought by the omphalo-mesaraic arteries finds its way into the lateral portions of the sinus terminalis, S.T., and there divides on each side into two streams. Of these, the two which, one on either side, flow backward, meet at a point about opposite to the tail of the embryo, and are conveyed along a distinct vein which, running straight forward parallel to the axis of the embryo, empties itself into the left omphalo-mesaraic vein. The two forward streams reaching the gap in the front part of the sinus terminalis fall into either one, or in some cases two veins, which run straight backward parallel to the axis of the embryo, and so reach the roots of the heart. When wne such vein only is present it joins the left omphalomesaraic trunk; where there are two they join the left and right omphalo-mesaraic trunks respectively. The left vein is always considerably larger than the right; and the latter when present rapidly gets smaller and speedily disappears.

The chief differences then between the peripheral circulation of the second and of the third day are due to the greater prominence of the sinus terminalis and the more complete arrangements for returning the blood from it to the heart. After this day, although the vascular area will go on increasing in size until it finally all but encompasses the 
yolk, the prominence of the sinus terminalis will become less and less in proportion as the respiratory work of the vascular area is shifted on to the allantois, and its activities confined to absorbing nutritive matter from the yolk.

4. The folding in of the embryo makes great progress during this day. Both head and tail have become most distinct, and the side folds which are to constitute the lateral walls have advanced so rapidly that the embryo is now a bona fide tubular sac, connected with the rest of the yolk by a broad stalk. This stalk, as was explained in Chap. II, is double, and consists of an inner splanchnic stalk continuous with the alimentary canal, which is now a tube closed at both ends and open to the stalk along its middle third only, and an outer somatic stalk continuous with the body-walls of the embryo, which have not closed nearly to the same extent as the walls of the alimentary canal. (Compare Fig. 8. $A$ and $B$, which may be taken as diagrammatic representations of longitudinal and transverse sections of an embryo of this period.)

5. The embryo is almost completely covered by the amnion. Before the close of the day the several amniotic folds will have met along a line over the back of the embryo. Their complete coalescence, and the obliteration of their line of junction, will however not take place till the following day.

6. During this day a most remarkable change takes place in the position of the embryo. Up to this time it has been lying symmetrically upon the yolk with the part which will be its mouth directed straight downwards. It now turns round so as to lie on its left side.

This important change of position is almost invariably completed on the third day. At the same time the left omphalo-mesaraic vein, the one on the side on which the embryo comes to lie, grows very much larger than the right, which henceforward gradually dwindles and finally disappears.

7. Coincidently with the change of position the whole embryo begins to be curved on itself. This curvature of the body, Fig. 46, becomes still more marked on the fourth day.

8. In the head very important changes take place. One of these is the cranial flexure, Figs. 24, 25. This (which 
must not be confouncied with the curvature of the body just referred to) we have alrealy seen was commenced in the course of the second day, by the bending downwards of the head round a point which may be considered as the extreme end either of the notochord or of the alimentary canal.

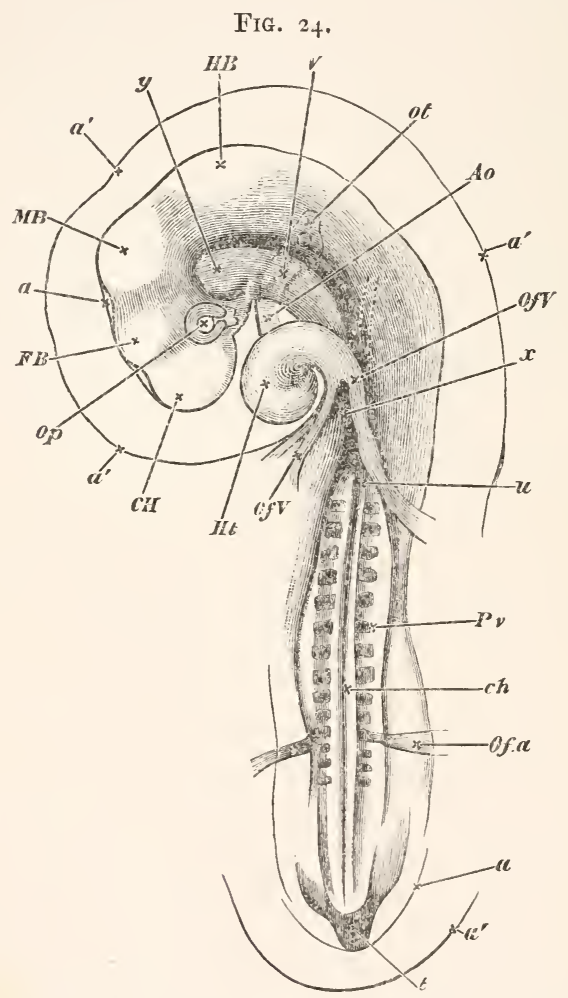

Chick of the Third Day ( $5+$ Hours) viewed from underneath as a Transparent OBJect.

$a^{\prime}$. the outer amniotic fold or false amnion. 'This is very conspicuous around the head, but may also be seen at the tail.

a. the true amnion, very closely enveloping the head, and here seen only between the prijuctions of the several cerebral vesicles. It may also be traced at the tail, $t$.

In the embryo of which this is a drawing the head-fold of the amnion reached a little farther backward than the reference $u$, but its limit could not be 
distinctly seen through the body of the embryo. The prominence of the false ammon at the head is apt to puzzle the student; but if he bears in mind the fact, which could not well be shewn in Fig. 8 , that the whole ammiotic fold, both the true and the false limb, is tucked in underneath the head, the matter will on reflection become intelligible.

$C . H$. cerebral hemisphere. $F . B$. fore-brain or vesicle of the third ventricle. M. B. mid-brain. H. B. hind-brain. Op. optic vesicle. Ot, otic vesicle.

$O f V$. omphalo-mesaraic veins forming the venous roots of the heart. The trunk on the right hand (left trunk when the embryo is viewed in its natural position from above) receives a large b:anch, shewn hy dotted lines, coming from the anterior portion of the sinus terminalis $H t$. the heart, now completely twisted on itself. Ao. the bulbus arteriosus, the three aortic arches being dimly seen stretching from it across the throat, and uniting into the aorta, sti.l more dimly seen as a curved dark line ruming along the body. The other curved dark line by its side, ending near the reference $y$, is the notochord $c h$.

About opposite the line of reference $x$ the aorta divides into two trunks, which running in the line of the somewhat opaque protovertebre on either side, are not clearly seen. Their branches however, $O f a$, the omphalo-mesaraic arteries, are conspicuous and are seen to curve round the commencing side folds.

Pv. protovertebræ. Below the level of the omphalo-mesaraic arteries the vertebral plates are but imperfectly cut up into protovertebiæ, and lower down still, not at all.

$x$ is placed at the "point of divergence" of the splanchnopleure folds. The blind foregut begins here and extends about up to $y$, the more transparent space marked by that letter being partly due to the presence there of the cavity of the alimentary canal. $x$ therefure marks the present hind limit of the splanchnopleure folds. The limit of the more transparent somatopleure folds cannot be suen.

It will be of course understood that all the body of the embryo above the level of the reference $x$, is seen through the portion of the yoli-sac (vascular and pellucid area), which has been removed with the embryo from the egg, as well as through the duble amniotic foid.

We may repeat that, the view being from below, whatever is described in the natural position as being to the right here appears to be left, and vice versa.

The flexure progresses rapidly, the front-brain being more and more folded down till, at the end of the third day, it is no longer the first ve:icle or fore-brain, but the second cerebral vesicle or mid-brain, which occupies the extreme front of the long axis of the embryo. In fact a straight line through the long axis of the embryo would now pass through the mid-brain instead of, as at the beginning of the second day, through the fore-brain, so completely has the front end of the nemral canal been folded over the end of the notochord. The commencement of this cranial flexure gives the body of an embryo of the third day somewhat the appearance of a retort, the head of the embryo corresponding 
to the bulb. On the fourth day the flexure is still greater than on the third, but on the fifth and succeeding days it becomes less obvious owing to the filling up of the parts of the skull.

FIG. 25 .

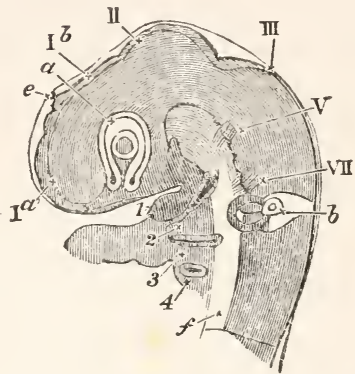

Head of a Chick of the third day viewed sidewars as a Transparent Овлест. (From Huxley.)

I. $a$. the vesicle of the cerebral hemisphere. I. $b$. the vesicle of the third ventricle (the original fore-brain); at its summit is seen the projection of the pineal gland $e$.

Below this portion of the brain is seen, in optical section, the optic vesicle $a$ alrearly involuted with its thick inner and thinner outer wall (the letter $a$ is placed on the junction of the two, the primary cavity being aimost obliterated). In the centre of the vesicle lies the lens, the shaded portion (represented too large), being the expression of its cavity. Below the lens between the two limbs of the horse-shoe is the choroidal fissure.

II. the mid-brain. now, owing to the cranial flexure, opposite the end of the alinentary canal. III. the hind-brain. V. the rudiments of the fifth cranial nerve, VII. of the seventh. Below the seventh nerve is seen the auditory vesicle $b$. The head having been subjected to pressure, the vesicle appear's somewhat distorted as if squeezed out of place. The orifice is not yet quite closed up.

1. the inferior maxillary process of the first visceral fold. Below, and to the right of this, is seen the first visceral cleft, below that again the second visceral fold (2), and lower down the third (3) and fourth (4) visceral folds. In front of the folds (i.c. to the left) is seen the arterial end of the heart, the aortic arches being buried in their respective visceral folds.

f. represents at its lowest part the cavity of the alimentary canal as seen through the transparent body of the embryo; at the upper part below the brain it is difficult to distinguish between the transparency due to the presence of the cavity of the alimentary canal, and that caused by the character of the mesoblast at the base of the skull, which, being formed of stellate cells with largely developed clear spaces or vacuoles, allows the light to pass readily through it. Near its upper end below the mid-brain is seen a small conical process, the rudiment of the infundibulum. 
on the second day began to grow out from the front of the fore-brain, increase rapilly in size luring the third day, so much so that by the end of the day each of them (Fig. 24, CH, Fig. 2.), I $九$ ) is as large or larger than the original fore-brain from which they both sprang, and they form together a most conspicuous part of the brain. In their growth they pash aside the optic vesicles, and thus contribute largely to the roundness which the head is now acquiring. Each vesicle possesses a cavity, known afterwards as a luteral ventricle, which, though quite separate from its fellow, is continuous with the cavity of the fore-brain.

Owing to the development of these cerebral hemispheres, the original fore-brain no longer occupies the front position (Fig. 24, FB, Fig. 25, Ib), and ceases to be the conspicuous object that it was. Inasmuch as its walls will hereafter be developed in to the parts surrounding the so-called third rentricle of the brain, we shall henceforward speak of it as the vesicle of the third ventricle, or, inasmuch as it soon comes to lie between the expanded posterior ends of the cerebral hemispheres, as the 'tween brain.

On the summit of the 'tween brain there may now be seen a small conical projection, the rudiment of the pineal gland (Fig. 25, e), while the centre of the floor is produced into a fumnel-shaped process, the infundibulum (Fig. $2 \cdot \boldsymbol{2}, d$ ), which, stretching towards the extreme end of the alimentary canal, joins the pituitary body.

The development of the pituitary body or hypophysis cerebri has been the subject of considerable controversy amongst embryologists. Von Baer (loc. cit.) and Smidt (Zeitschrift für IV'iss. Zoologie, 1862, B. XI, p. +3) believed that the base of the fore-hrain, or vesicle of the third ventricle, became produced into a downward process, the 'infundibulum,' which subsequently became expanded at its termination to form the pituitary body.

Ratlike (Avehiv fiir Anatomie und Plyysiologie $1838, \mathrm{Bd}$. v.) states that very early a diverticulum is produced from the upper end of the alimentary canal, which passes backwards and meets the process of the brain called the inflindibulum. This diverticulum subsequently loses all connection with the epithelium of the digestive canal, and, uniting with the infundibulum, forms the pituitary body.

Dursy (Entwieklungsgesclielte des Kopfes, Tübingen, 1869) states that both the end of the notochord and the epithelium of the alimentary canal take part in the formation of the pituitary body. The apparent diverticulum of the alimentary canal is not so much a true diverticulum, as a part of the alimentary can:l constricted off from the remainder by the cranial flexure.

Reichert (Entuicklungsleben im Wirbelthierreich. Berlin, 18+0) states that the pituitary body is formed from the remains of the front end of notochord. 
Subsequently however (Der Bau des menschlichen Gehirns) he supposed that it was formed from the pla mater.

Rathke also subsequently (Entuicklunsgeschichte der Wirbelthiere, Leipzig, 1861) gave up his former view, and believed that the diverticulum of the alimentary canal disappeared, but that the pituitary body was formed from the mesoblast in front of the clinoil process.

Wilhelm Miiler (Leber die Enturirklung und Bau der Hypophysis und des Processus infundibuli cerebri. Jenctische Zeitschrift, Bd. vг. 1871) has recently written an elaburate memoir on the deve opment and anatomy of the pituitary body and infundibulum in all the orders of Vertebrates, of which the following is an abstract.

In order to understand the formation of the diverticulum from the alimentary canal which forms the pituitary body, we must remember that at first the hypoblast of the throat closely underlies the notochord, and beyond the end of the notochord is almost in contact with the base of the vesicle of the third ventricle. When the cranial flexure occurs, which it will be remembered takes place about an axis conciding with the end of the notochord, the hypoblast, which closely underlies the base of the brain, becomes at the same time bent; and as the angle of the flexure becomes an acute angle, a wedgeshaped space lined by hypoblast is as it were constricted off from the alimentary canal. In this way there is formed a divericulum of hypoblast which passes forwards from the alimentary canal to the base of the fore-braina diverticulum not produced by a forward growth from the alimentary canal, but solely due to the cranial flexure constrictin: off a wedge-shaped portion of the alimentary cinal. This we may call the pituitary diverticulum. When the cranial flexure commences the end of the notochord becomes bent downward, and, ending in a somewhat enlarged extremity, comes in contact with the termination of the pituitary diverticulum. The mesoblast around and at the front of the end of the notochord increases and grows up, in front of the notochord and behind the vesicle of the third ventricle, to form the posterior clinoid process. The base of the vesicle of the third ventricle at the same time grows downwards towaris the pituitary diverticulum and forms what is known as the infundibulum. This state of things may be observed on the third day. On the fourth day the mesoblast tissue around the notochord increases in quantity, and the end of the notochord, though still bent downwards, recedes a little from the termination of the pituitary diverticulum, which is still a triangular space with a wide opening into the alinentary canal.

On the fifth day, the opening of the pituitary diverticulum into the alimentary canal has become narrowed, and around the whole diverticulum a furmation of mesolblast-cells has commenced. Behind it the clinoid process has become cartilaginous, while to the side and in front it is encloserl by the trabeculæ. At this stage, in fact, we have a diverticulum from the alimentary canal passing through the base of skull to the infundibulum. The end of the notochord has at this stage become atrophied, so that it is separated by a considerable interval from the pituitary body.

On the seventl diay the mesoblast arnund the pituitary diverticulum has grown into a complete investment of spindle-shlaperl cells, and the communication between the cavity of the diverticulum and that of the throat has become still narrower. The diverticulum is all but converted into a vesiele, and its hypoblast walls have commencerl to send out into the mesoblastic investment solid processes, which form the first commencement of the true pituitary body. The infundibulum now appears as a narow process from the base of the vesicle of the third ventricle, which approaches, but does not unite with the pituitary vesicle. This latter lies in the spatce between the basi- and the presphenoid, and is 
completely surrounded by a ring of cartilage. The mesoblast-cells immediately around it do not, hwwerer, exhibit any signs of becoming cartilaginous.

By the tenth day the opening of the pituitary vesicle into the thrcat becomes almost obliterated, and the lumen of the vesicle itself very much diminished. The body itself consists of anastomosing cords of hyp iblast-cells, the mesoblast between which has already commenced to become vascular. The cords or masses of hypoblast cells are surrounded by a delicate membrana propria, and a few of them possess a small lumen. The infundibulum has increased in lenџth.

On the twelfth day the communication between the pituitary vesicle and the thrnat is entirely obliterated, but a sulid cord of cells still connects the two. The vessels of the pia mater of the vesicle of the third ventricle have become connected with the pituitary body, and the infundibulum has grown down along its posterior border.

In the later stages, all connection is lust between the pituitary body and the throat, and the former becomes connected with the elongated processus infundibuli.

Such is Wilhelm Müller's account. Goette, however (Archir. Micr. Anat. IX. p. 397), has recently given reasons for thinking that the pituitary diverticulum arises not from the closed foregut, lined 1 ith hypoblist, but from the buccal cavity lined with epiblast. He states that in its earlier stages it may be seen to start on the oral sile of the partition, which for some time divides the secondarily formed buccal cavity from the primarily formed foregut, and therefore, belonging to the furmer, cannot be regarded as the natural anterior termination of the latter.

Beyond an increase in size, which it shares with nearly all parts of the embryo. and the change of position to which we have already referred, the mid-brain undergoes no great alteration during the third day. Jts roof will ultimately become developed into the corpora bigemina or optic lobes (quadrigemina in mammals), its floor will form the crura cerebri, and its cavity will be reduced to the narrow canal known as the iter a tertio ad quartum ventriculum.

In the hind-brain, or third cerebral vesicle, that part which lies nearest to the mid-brain, becomes during the third day marked off from the rest by a slight constriction. This distinction, which becomes much more evident later on by a thickening of the walls and roof of the front portion, separates the hind-brain into the cerebellum in front, and the medulla oblongata behind. While the walls of the cerebellar portion of the hind-brain become very much thickened as well at the roof as at the floor and sides, the roof of the posterior or medulla oblongata portion thins out into a mere membrane, forming a delicate covering to the carity of the resicle (Fig. 26. IV), which here becoming broad and shallow with greatly thickened floor and sides, is 
known as the fourth ventricle, subsequently overhung by the largely developed posterior portion of the cerebellum.

The third day, therefore, marks the distinct differentiation of the brain into its fundamental parts: the cerebral hemispheres, the central masses round the third ventricle, the corpora bigemina, the cerebellum and the medulla oblongata; the original cavity of the neural canal at the same time, passing from its temporary division of three single cavities into the permanent arrangement of a series of connected ventricles, viz. the lateral ventricles, the third ventricle, the iter (with a prolongation into the optic lobe on each side), and the fourth ventricle.

10. At the same time that the outward external shape of the brain is thus being moulded, interual changes are taking place in the whole neural canal. These are best seen in sections.

At its first formation, the section of the cavity of the neural canal is round or nearly so.

About this time, however, the lining of involuted epiblast along the length of the whole spinal cord becomes very much thickened at either side, while increasing but little at the mid-points above and below. The result of this is that the cavity as seen in section (Fig. 44), instead of being circular, has become a narrow vertical slit, almost completely filled in on either side.

In the region of the brain the thickening of the lining epiblast follows a somewhat different course. While almost everywhere the sides and floor of the canal are greatly thickenced, the roof in the region of the various ventricles, not of the fourth only, but of the others as well, becomes excessively thin, so as to form a membrane reduced to almost a single layer of cells. (Fig. 26. IV.)

11. In the preceling chapter we saw how the first cerebral vesicle, by means of lateral outgrowths followed by constrictions, gave rise to the optic vesicles. These and the parts surrounding them undergo on the third day changes which result in the formation of the eyebull.

At their first appearance the optic vesicles stand out at nearly right augles to the long axis of the embryo (Fig. 15), and the stalks which connect them with the fore-brain are short and wide. We have already said (p. 77) that the con- 
strictions which give rise to the stalks take place chiefly from above downwards, and also somewhat inwards and backwards. 'Thus from the first the vesicles appear to spring from the under part of the fore-brain.

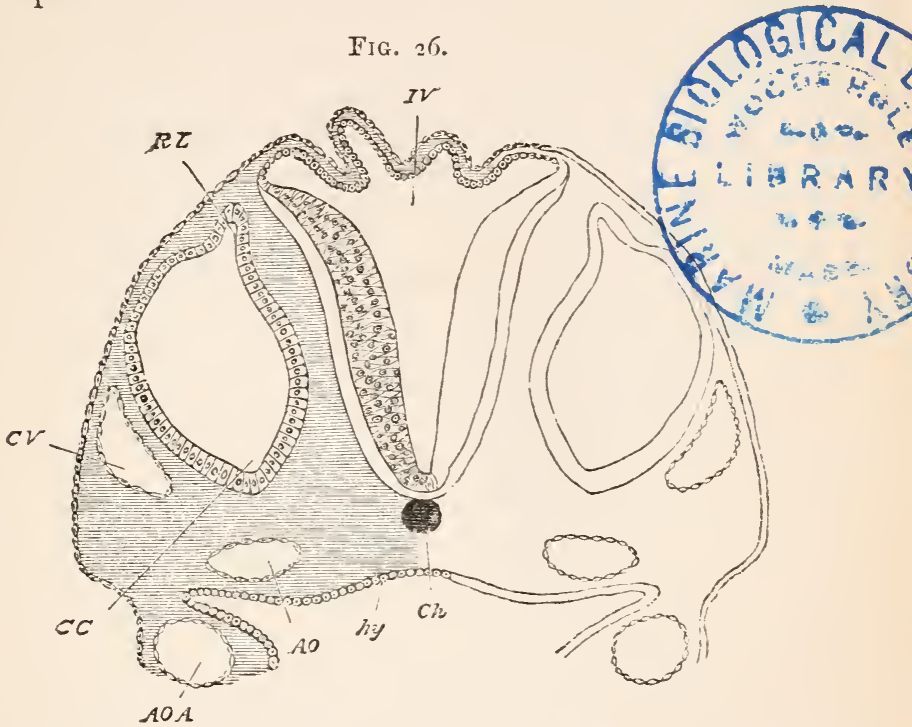

Sectiox throvgh the Hind-Bratn of a Chick at the end of the Third Day of Incubation.

$I V$. Fourth ventricle. The section shews the very thin roof and thicker sides of the ventricle.

Ch. Notochord--(diagrammatic shading).

$C V$. Anterior cardinal vein.

$C C$. Involuted auditory vesicle. $C C$ points to the end which will form the cochlear canal. $R L$. Recessus labyrinthi. hy. hypoblast lining the alimentary canal. $h y$ is itself placed in the cavity of the alimentary canal, in that part of the canal which will become the throat. The lower (anterior) wall of the canal is not shewn in the section, but on each side are seen portions of a pair of visceral arches. In each arch is seen the section of the aortic arch $A O A$ belonging to the visceral arch. The vessel thus cut through is running upwards towards the head, being about to join the dorsal aorta AO. Had the section been nearer the head, and carried through the plane at which the aortic arch curves round the alimentary canal to reach the mesoblast above it, $A O A$ and $A O$ would have formed one continuous curved space. In sections lower down in the back the two aorta, $A O$, one on either side would be found fused into one median canal.

The shading of the mesoblast is diagrammatic; it is here a uniform mass of spindle-shaped cells, there being in this region no differentiatiun into protuvertebræ. 
As the vesicles of the cerebral hemispheres grow out rapidly from the front and under portions of the first cerebral vesicle, they seem to thrust the optic vesicles apart and to the sides.

Thus these, instead of standing out from the extreme front, come to be placed at the sides of the head, the stalks, which are correspondingly lengthened and narrowed, running obliquely downwards and inwards from the vesicles to open into the cavity of the brain at its base. Their openings are at first placed close to each other at the junction of the cerebral hemispheres with the remnant of the fore-brain (now called the vesicle of the third ventricle), so that the cavities of the two optic vesicles may be said to communicate directly both with each other and with the cavities of the cerebral hemispheres. The later connection is however soon lost, and the stalks of the optic vesicles then open exclusively into the third ventricle. At the same time the floor of the third ventricle, during the occurrence of the cranial flexure, grows down and thrusts apart the openings of the two optic stalks. At a later date the stalks shift their position backwards, and thus become connected chiefly with the base of the midbrain.

While these changes have been going on in the optic stalks, development has also proceeded in the region of the vesicles themselves, and given rise to the rudiments of the retina, lens, vitreous humour, and other parts of the eye.

The changes through which these are formed are of a somewhat complicated character, and not a few points in reference to them are still involved in some doubt.

Towards the end of the second day, the external or superficial epiblast which covers, and is in all but immediate contact with the most projecting portion of the optic vesicle, becomes thickened. 'This thickened portion is then driven inwards in the form of a shallow open pit with thick walls (Fig. $27 A . x$ ), carrying before it the front wall $(r)$ of the optic vesicle. To such an extent does this involution of the superficial epiblast take place, that the front wall of the optic vesicle is pushed close up to the hind wall, and the cavity of the vesicle becomes almost obliterated (Fig. 27, $B$ ).

The bulb of the optic vesicle is thus converted into a cup with double walls, containing in its cavity the portion of 
involuted epiblast. This cup, in order to distinguish its cavity from that of the original optic vesicle, is generally called the secondary optic vesicle. We may, for the sake of brevity, speak of it as the optic cup; in reality it never is a vesicle, since it always remains widely open in front. Of its double walls the inner or anterior (Fig. $27 \mathrm{~B}, r$ ) is formed from the front portion, the outer or posterior (Fig. 27 $B, u$ ) from the hind portion of the wall of the primary optic vesicle. The inner or anterior $(r)$, which very speedily becomes thicker than the other, is converted into the retina; in the outer or posterior $(u)$, which remains thin, pigment is eventually deposited, and it ultimately becomes the tesselated pigment-layer of the choroid.

Fig. 27.

A.

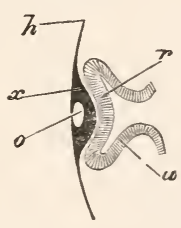

$B$.

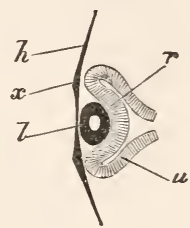

Diagramiratic Sections illustrating the Formation of the Eye.

(After Remak.)

In $A$, the thin superficial epiblast $h$ is seen to be thickened at $x$, in front of the optic vesicle, and involuted so as to form a pit $o$, the mouth of which has already begun to close in. Owing to this involution, which forms the rudiment of the lens, the optic vesicle is doubled in, its front portion $r$ being pushed against the back portion $u$, and the original cavity of the vesicle thus reduced in size. The stalk of the vesicle is shewn as still broad.

In $B$, the optic vesicle is still further doubled in so as to form a cup with a posterior wall $u$ and an anterior wall $r$. In the hollow of this cup lies the lens $l$, now completely detached from the superficial epiblast $x, h$. Its cavity is still shewn. The cavity of the stalk of the optic vesicle is already much narrowed.

By the closure of its mouth the pit of involuted epiblast hecomes a completely closed sac with thick walls and a small central cavity. (Fig. $27 B, l$ ). At the same time it breaks away from the external epiblast, which forms a continuous layer in front of it, all traces of the original opening being lost. There is thus left lying in the cup of the secondary optic vesicle, an isolated elliptical mass of epiblast. This is 
the rudiment of the lens. The small cavity within it speedily becomes still less by the thickening of the walls, especially of the hinder one.

At its first appearance the lens is in immediate contact with the anterior wall of the secondary optic vesicle (Fig. $\because 7 B)$. In a short time however, the lens is seen to lie in the mouth of the cup (Fig. 30 D), a space (vh) (which is subsequently occupied by the vitreous humour) making its appearance between the lens and anterior wall of the vesicle.

In order to understand how this space is developed, the position of the optic vesicle and the relations of its stalk must be borne in mind.

FIG. 28.

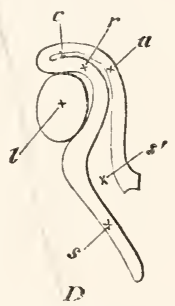

Diagramatic Section of the Eye and the Optic Nerve at a EARLY STAGE (from Lieberkühn),

to shew the lens $l$ occupying the whole hollow of the optic cup, the inclination of the stalk $s$ to the optic cup, and the continuity of the cavity of the stalk $s^{\prime}$ with that of the primary vesicle $c$; $r$, anterior, $u$ posterior wall of the optic cup.

The vesicle lies at the side of the head, and its stalk is directed downwards, inwards and backwards. The stalk in fact slants away from the vesicle. Hence when the involution of the lens takes place, the direction in which the front wall of the vesicle is pushed in is not in a line with the axis of the stalk as for simplicity's sake has been represented in the diagram Fig. 27 , but forms an obtuse angle with that axis, after the manner of Fig. 28, where $s^{\prime}$ represents the cavity of the stalk leading away from the almost obliterated eavity of the primary vesicle.

Fig. 28 represents the early stage at which the lens fills the whole cup of the secondary vesicle. The subsequent state of affairs is brought about through the growth of the 
walls of the cup taking place more rapidly than that of the lens, or in other words to the cavity of the cup dilating. But this growth or this dilatation does not take place equally in all parts of the cup. The walls of the cup rise up all round except that point of the circumference of the cup which is opposite the middle (from side to side) of the stalk. While elsewhere the walls increase rapidly in height, carrying so to speak the lens with them, at this spot, which in the natural position of the eye is on its under surface, there is no growth: the wall is here imperfect, and a gap is left. 'Through this gap, which afterwards receives the name of the choroidal fissure, a way is open from the mesoblastic tissue surrounding the optic vesicle and stalk into the interior of the cavity of the cup.

FIG. 29.

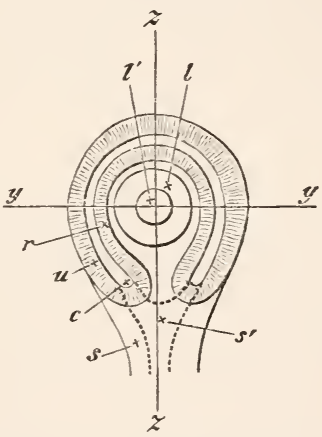

Diagramatic Representation of the Eye of the Chick of about the THIRD DAY AS SEEN WHEN THE HEAD IS VIEWED FROM UNDERNEATH AS A TRANSPARENT OBJECT.

$l$ the lens, $l^{\prime}$ the cavity of the lens, lying in the hollow of the optic cup.

$r$ the anterior, $u$ the posterior wall of the optic cup, $c$ the cavity of the primary optic vesicle, now nearly obliterated. By inadvertence $u$ has been drawn in some places thicker than $r$, it should have been thinner throughout.

$s$ the stalk of the optic cup with $s^{\prime}$ its cavity, at a lower level than the cup itself and therefore out of focus; the dotted line indicates the continuity of the cavity of the stalk with that of the primary vesicle.

The line $z, z$, through which the section shewn in Fig. $30 \mathrm{~F}$ is supposed to be taken, passes through the choroidal fissure.

From the manner of its formation the gap or fissure is evidently in a line with the axis of the optic stalk, and in 
order to be seen must be looked for on the under surface of the optic vesicle. In this position it is readily recognized in the transparent embryo of the third day, Figs. 25 and 29.

Bearing in mind these relations of the gap to the optic stalk, the reader will understand how sections of the optic vesicle at this stage present very different appearances according to the plane in which the sections are taken.

When the head of the chick is viewed from underneath as a transparent object the eye presents very much the appearance represented in the diagram Fig. 29.

\section{FIG. 30.}

D

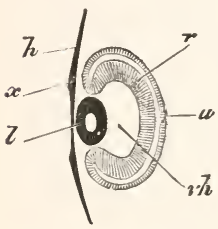

F.

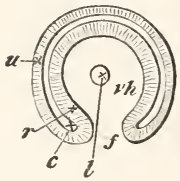

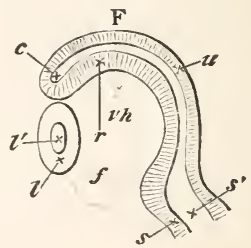

D. Diagrammatic section taken perpendicular to the plane of the paper, along the line $y, y$, Fig. 29. The stalk is not seen, the section falling quite out of its region. $v h$, hollow of optic cup filled with vitreous humour; other letters as in Fig. $27 \mathrm{~B}$.

E. Section taken parallel to the plane of paper through Fig. 29, so far behind the front surface of the eye as to shave off a small portion of the posterior surface of the lens $l$, but so far in front as not to be carried at all through the stalk. Letters as before; $f$, the choroidal fissure.

F. Section along the line $z$, $z$, perpendicular to the plane of the paper, to shew the choroidal fissure $f$, and the continuity of the cavity of the optic stalk with that of the primary optic vesicle. Had this section been taken a little to one side of the line $z, z$, the wall of the optic cup would have extended up to the lens below as well as above. Letters as before.

A section of such an eye taken along the line $y$, perpendicular to the plane of the paper, would give a figure corresponding to that of Fig. $30 \mathrm{D}$. The lens, the cavity and double walls of the secondary vesicle, the remains of the primary cavity, would all be represented (the superficial epiblast of the head would also be shewn); but there would be nothing seen of either the stalk or the fissure. If on the other hand the section were taken in a plane parallel to the plane of the paper, at some distance above the 
level of the stalk, some such figure would be gained as that shewn in Fig. 30 E. Here the fissure $f$ is obvious, and the communication of the cavity $v h$ of the secondary vesicle with the outside of the eye evident; the section of course would not go through the superficial epiblast. Lastly, a section, taken perpendicular to the plane of the paper along the line $z$, i.e. through the fissure itself, would present the appearances of Fig. $30 \mathrm{~F}$, where the wall of the vesicle is entirely wanting in the region of the fissure marked by the position of the letter $f$.

The fissure such as we have described it exists for a short time only. Its lips come into contact, and unite (in the neighbourhood of the lens, directly, but in the neighbourhood of the stalk, by the intervention of a structure which we shall describe presently), and thus the cup-like cavity of the secondary optic vesicle is furnished. with a complete wall all round. The interior of the cavity is filled by the vitreous humour, a clear fluid in which are a few scattered cells.

In the foregoing account of the formation of the secondary optic vesicle, and of the fissure, as the results of a process of unequal growth, we have followed the account of Lieberkühn (Über das Auge des Wirbelthierembryos, Schriften der Gesellschaft zur Beförderung der gesammten Naturwissenschaften zu Marburg. Bd. I0. 1872). Their origin is more generally described as being due to a doubling up of the primary vesicle from the side along the line of the fissure at the same time that the lens is being thrust in in front. In mamınalia, the doubling up is said to involve the optic stalk, which becomes flattened (whereby its original cavity is obliterated) and then folded in on itself, so as to embrace a new central cavity continuous with the cavity of the vitreous humour.

According to Lieberkühn the optic stalk in birds is never so folded up, but is converted into the optic nerve by the gradual obliteration of its primary central cavity through increased thickening of the walls. The optic nerve of the bird, moreover, contains no arteria centralis retinæ, while the involution of the optic stalk into the optic nerve was supposed to have for its purpose the introduction of a quantity of mesoblast into the interior of nerve, in order to form the artery.

According to Remak and the majority of observers after him, no mesoblast whatever exists between the external epiblast and the optic vesicle, at the point where the former is thrust inwards to form the lens, and hence this organ carries with it in its involution no mesoblast whatever to serve as a rudiment of either the vitreous humour or the capsule of the lens. They described the vitreous humour as being formed entirely out of the mesoblast which was intruded from the exterior of the eye through the choroidal fissure, and Kölliker considered the capsule of the lens as a sort of cuticular excretion from the surface of the lens itself. Lieberkühn on the other hand states that shortly after the commencement of the involution of the lens there 
may be already found a thin layer of mesoblast, interposed between it and the optic vesicle. This layer is carritd inward during the involution, and from it both the vitreous humour and the capsule of the lens take their origin. In hirds it is very difficult to be sure of the existence of this layer, though Lieberkiihn says that in mammals it is conspicuous; and even if its existence be admitted, it still remains doubtful whether it gives rise to the whole vitreous hmmour, or to the capsule of the lens only; though the latter view is most 1 robable.

During the changes in the optic vesicle just described, the surrounding mesoblast takes on the characters of a distinct investment, whereby the outline of the eyeball is definitely formed. The internal portions of this investment, nearest to the retina, become the choroid (i.e. the chorio-capillaris, and the lamina fusca, the pigment epithelium, as we have seen, being derived from the epiblastic optic cup), and pigment is subsequently deposited in it. The remaining external portion of the investment forms the sclerotic.

The complete differentiation of these two coats of the eye does not however take place till a late period. Along the line of the choroidal fissure the pigment is wanting. Consequently in embryos of an age when the pigment has become generally deposited in the choroid, a colourless streak marking out the position of the choroidal fissure is very conspicuous.

In front of the optic cup the mesoblastic investment grows forwards, between the lens and the superficial epiblast, and so gives rise to the substance of the cornea; the epiblast supplying only the anterior epithelinm.

At first the whole space between the lens and the superficial epiblast is occupied by undifferentiated mesoblast; but on the sixth day a layer of epithelium makes its apparance in midst of the mass, and thus divides it into an anterior and a posterior portion. The anterior portion, increasing in solidity, becomes the cornea, and remains continuous with the sclerotic; the epithelium in question persisting as the posterior epithelium of the membrane of Descemet. The posterior portion is reduced to a mere membrane forming, accorling to Lieberkiilnn, the front limb of the capsule (and the suspensory ligament) of the lens, the space between it and the cornea becoming filled with aqueous humour.

We left the original cavity of the primary optic vesicle as 
a nearly obliterated space between the two walls of the optic cup. By the end of the third day the obliteration is complete, and the two walls are in immediate contact.

The inner or anterior wall is, from the first, thicker than the outer or posterior; and over the greater part of the cup this contrast increases with the growth of the eye, the anterior wall becoming markedly thicker and undergoing changes of which we shall have to speak directly (Fig. 31).

In the front portion however, along, so to speak, the lip of the cup, anterior to a line which afterwards becomes the ora serrata, both layers not only cease to take part in the increased thickening, accompanied by peculiar histological changes, which the rest of the cup is undergoing, but also completely coalesce together. Thus a hind portion or true retina is marked off from a front portion.

The front portion, accompanied by the choroid which immediately overlays it, is behind the lens thrown into folds, the ciliary ridges; while further forward it bends in between the lens and the cornea to form the iris. The original wide opening of the optic cup is thus narrowed to a smaller orifice, the pupil ; and the lens, which before lay in the open mouth of, is now inclosed in the cavity of the cup. While in the hind portion of the cup or retina proper, no deposit of black pigment takes place in the layer formed out of the inner or anterior wall of the vesicle, in the front portion we are speaking of, pigment is largely deposited throughout both layers, so that eventually this portion seems to become nothing more than a forward prolongation of the pigmentepithelium of the choroid.

Thus while the hind moiety of the optic cup becomes the retina proper, including the choroid-pigment in which the rods and cones are imbedded, the front moiety is converted into the ciliary portion of the retina, covering the ciliary processes, and into the uvea of the iris; the bodies of the ciliary processes and the substance of the iris, their vessels, muscles, connective tissue and ramified pigment, being derived from the mesoblastic choroid. The margin of the pupil marks the extreme lip of the optic vesicle, where the outer or posterior wall turns round to join the inner or anterior.

We have still to speak of the choroidal fissure. In mammals the slit remains open for a short time only. After 
Fig. 3I.

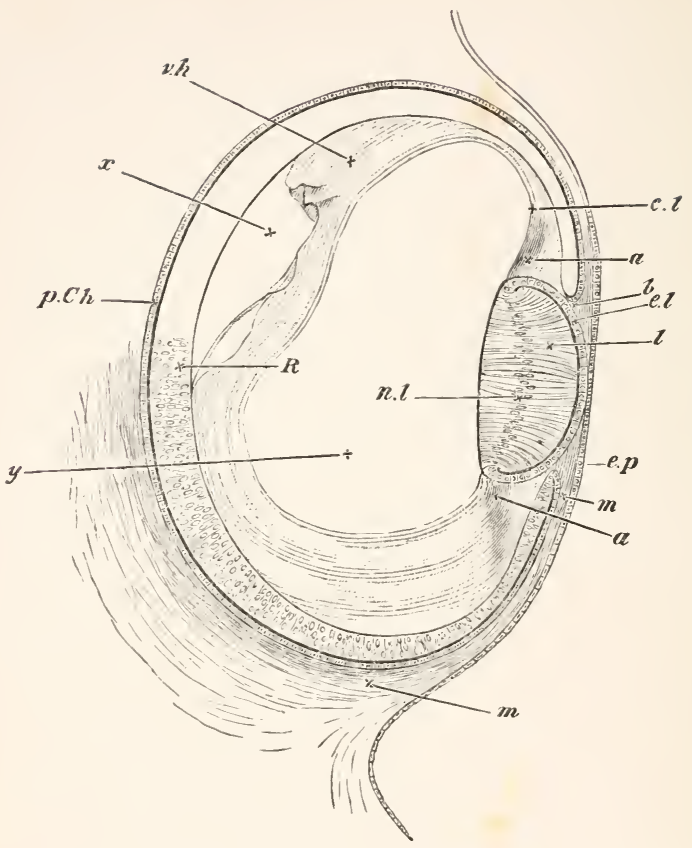

Section of the Eye of Chick at the Fourth Day.

є. p. superficial epiblast of the side of the head.

$R$. true retina: anterior wall of the optic eup. $p$. Ch. pigment-epithelium of the choroid : posterior wall of the optic cup. $b$ is placed at the extreme lip of the optic cup at what will become the margin of the pupil.

1. the lens. The hind wall, the nuclei of whose elongated cells are shewn at $n l$, now forms nearly the whole mass of the lens, the front wall being reduced to a layer of flattened cells $e l$.

$m$. the mesoblast surrounding the optic cup and about to form the choroid and sclerotic. It is seen to pass forward between the lip of the optic cup and the superficial epiblast.

Filling up a large part of the hollow of the optic cup is seen a hyaline mass $v h$, possibly the rudiment of the vitreous humour. It has fallen away from the retina at $x$, and is also (apparently accidentally) wanting at $y$. In the neighbourhood of the lens it seems to be continuous as at $c l$ with the tissue $a$, which in turn is continuous with the mesoblast $m$, and appears to be the rudiment of the capsule of the lens and suspensory ligament. 
the formation of the vitreous humour within the cup, the edges of the slit grow completely together, and all traces of the seam disappear. In birds the course of events is somewhat different.

In addition to such amount of mesoblast as may pass through the slit to form the vitreous humour, two special processes of mesoblast grow in, one in the neighbourhood of the optic stalk, in the region of the true retina, and a second, which speedily becomes highly vascular, in that portion of the slit which corresponds to the ciliary part of the retina. The former process remains as the pecten so characteristic of the avian eye, while the latter vascular process serves to supply the pecten with blood.

By the twelfth day the fissure completely closes up and disappears between these two processes and also in front of the vascular one; but both the pecten and the vascular process are left projecting into the interior of the eye. Hence in the adult eye, the pecten seems to perforate the retina close to the entrance of the optic nerve, the nervous fibres of the retina spreading away in a radiate manner from it.

The optic stalk, which, as we have said, by an obliteration of its central canal becomes converted into the optic nerve, is at first equally continuous with the inner and with the outer wall of the retina. This must of necessity be the case, since the interval which primarily exists between the two walls is continuous with the cavity of the stalk (vide Figs. 28 and $\left.30 \mathrm{~F}, s^{\prime}\right)$. When the fibres however make their appearance in the nerve, they are found to be connected with the inner wall, or functional retina, only.

The histological condition of the eye in its earliest stages is very simple. Both the epiblast forming the walls of the optic vesicle, and the superficial layer which is thickened to become the lens, are composed of several layers of simple columnar cells. The surrounding mesoblast is made up of cells whose protoplasm is more or less branched and irregular. These simple elements are gradually modified into the complicated tissues of the adult eye, the changes undergone being most marked in the cases of the retina, the optic nerve, and the lens with its appendages.

The retina. At first the two walls of the optic cup do not greatly differ in thickness. On the third day the outer or 
posterior becomes much thinner than the inner or anterior, and by the middle of the fourth day is reduced to a single layer of fiattened cells (Fig. 31, p. C \%.). At about the 80th hour its cells commence to receive a deposit of pigment, and eventually form the so-called pigmentary epithelium of the choroid; from them no part of the true retina (or no other part of the retina, if the pigment-layer in question be supposed to belong more truly to the retina than to the choroid) is derived.

On the fourth day, the inner (anterior) wall of the optic cup (Fig. 31, $R$ ) is perfectly uniform in structure, being conposed of elongated somewhat spindle-shaped cells, with distinct nuclei. On its external (posterior) surface a distinct cuticular membrane, the membrana limitans externa, early appears.

As the wall increases in thickness, these cells multiply rapidly, so that the wall becomes several cells thick.

The first indications of a division into layers are noticed on the seventh day; and on the eighth day a layer of 'granules' is very obvious. The granules, which are apparently nuclei of cells. become on the tenth day distinctly arranged into an inner and an onter layer; and at about the same time small processes, apparently outgrowths from the outer granular layer, make their appearance on the external surface of the membrana limitans externa. These processes are the rudiments of the rods and cones.

From the first they may be roughly divided into two categories, (I) those of smaller, (2) those of larger diameter. Both kinds grow rapidly and in the tips of both small highly refractive globules soon appear. The thinner processes are the cones, the thicker the rods. The cones remain for a long time thinner than the rods, but shortly hefore the exclusion of the chick they increase rapidly in diameter and soon after that oecurrence are found to surpass the rods in thickness. On the Isth day some of the globules in the cones become red, on the I th $^{\text {th }}$ others become yellow, and very soon all the globules in the cones acquire a distinct colour. The globules in the rods remain uncoloured. The rods and cones then are outgrowths through the membrana limitans externa, from the inner wa'l of the optic cup or retina into the outer wall or pigment. epithelium of the choroid.

Remak and some other investigators were of opinion that the outer wall of the optic cup gave rise to the rods and cones as well as to the pigment-epithelim. The observations hivever of Max sichultze, Archir Micros. Anat. Iv. 1. 239, supported by Bahuehin, Wiirz. Nat. Zeitsch. IV. (1863) p. 7 I, and others, have clearly shewn that Remak's views were erroneous.

On the thirteenth day the molecular layer and the gan- 
glionic layer are distinguishable. Very early the substance of certain of the cells takes on the appearance of fibres, arranged vertically, i.e. radiating from the inner or anterior surface of the retina to the membrana limitans externa. These are the rudiments of the fibres of Miiller.

Thus of the cells of the inner wall of the cup, some become ganglionic cells, and others the fibres of Miiller, while the nuclei of yet others remain as the inner and onter granules. The rods and cones are outgrowths of the cells to which the outer granules belong. All parts of the retina, in fact, whether simply connective, or really nervous in nature, seem to be derived from epiblastic cells.

The changes described above are confined to that portion of the retina which lies behind the ora serrata. In front of this both walls of the cup coalesce as we have said into a cellular layer in which a deposit of pigment takes place.

The optic nerve. Histological changes are first observable in the optic stalk at about the time when its cavity loses all connection with the cerebral hemisphere and opens exclusively into the third ventricle. It is then that fibres first make their appearance in its walls, nuclei being still abundantly present. 'The stalk though much elongated is still hollow and its cavity is circular in section. According to Lieberkühn at no time does it (in the bird) undergo any involution tending to obliterate its cavity.

Soon after the deposition of pigment in the outer wall of the optic cup, while the optic stalks are as yet still hollow, the rudiments of the optic chiasma appear. The fibres of the one stalk grow over into the attachment of the other. About the same time the fibres at the neck of the optic cup grow forward and become connected with the retina, over whose internal surface they spread. The entrance of the optic nerve into the eyeball is closely connected with that of the pecten, its fibres passing in at the lower end of that body, coursing along its sides to its upper end and radiating from it as from a centre to all parts of the retina.

Before the exclusion of the chick the optic nerve becomes solid by the gradual filling up of its central cavity.

The lens when first formed is somewhat elliptical in section with a small central cavity of a similar shape, the front and hind walls being of nearly equal thickness, 
each consisting of a single layer of elongated columnar cells.

In the subsequent growth of the lens, the development of the hind wall is of a precisely opposite character to that of the front wall. The hind wall becomes much thicker, and tends to obliterate the central cavity by becoming convex on its front surface. At the same time its cells, still remaining as a single layer, become elongated and fibre-like. The front wall on the contrary becomes thinner and thinner and its cells more and more flattened and pavement-like.

These modes of growth continue until at the end of the fourth day, as shewn in Fig. 31, the central hind wall $l$ is in absolute contact with the front wall $e l$ and the cavity thus becomes entirely obliterated. The cells of the hind wall have by this time become veritable fibres which when seen in section appear to be arranged nearly parallel to the optic axis, their nuclei $n l$ being seen in a row along their middle. The front wall, somewhat thickened at either side where it becomes continuous with the hind wall, is now a single layer of flattened cells separating the hind wall of the lens, or as we may now say the lens itself, from the front limb of the lens-capsule; of this it becomes the epithelium.

The subsequent changes undergone consist chiefly in the continued elongation and multiplication of the lens-fibres, with the partial disappearance of their nuclei.

During their multiplication they become arranged in the manner so characteristic of the adult lens.

The lens-capsule and its adjuncts. In spite of the numerous investigations which have been made in reference to the development of the lens-capsule, its precise mode of origin can hardly even yet be said to be certainly known. Remak was led from analogy to regard it as a product of the mesoblast, though he did not succeed in satisfactorily demonstrating the fact. Kölliker looked upon it as a cuticular membrane thrown off by the superficial cells of the lens, and his view has been very generally adopted.

Lieberkühn has given a different account of its origin. According to him the involution of the lens, as we have already stated, carries inwards with it a very thin layer of mesoblast. 'This remains continuous with the mesoblast surrounding the eyeball, so that when subsequently the mesoblast 
grows forward over the front of the lens, the latter receives a complete mesoblastic investment.

Of this mesoblast a very thin layer next to the lens both in front and behind becomes separated from the rest, and forms the lens-capsule and suspensory ligament. The remainder of the mesoblast behind the lens becomes converted into the vitreous humour, the layer immediately in contact with the retina giving rise to the hyaloid membrane. That the hyaloid is really a product of the mesoblast and not a cuticular outgrowth from the epiblastic cells of the retina is indicated by the fact that it is continuous over the pecten, where of course the retina is absent. At its first appearance the vitreous humour is a mass of stellate cells; while however it is rapidly enlarging to fill up the growing optic cup, a large portion of it becomes entirely fluid, the cellular elements being more and more restricted to the immediate neighbourhood of the posterior surface of the lens, where a few stellate cells may be seen even in the adult.

Briefly to recapitulate. The eye commences as a lateral outgrowth of the fore-brain, in the form of a stalked resicle.

The stalk becoming narrowed and subsequently solid, is converted into the optic nerve.

An involution of the superficial epiblast over the front of the optic vesicle, in the form first of a pit, then of a closed sac with thick walls, and lastly, of a solid rounded mass (the small central cavity being entirely obliterated by the thickening of the hind wall), gives rise to the lens. Owing to this involution of the lens, the optic vesicle is doubled up on itself, and its cavity obliterated; thus a secondary optic vesicle or optic cup with a thick anterior and a thin posterior wall is produced. As a result of the manner in which the doubling up takes place, or of the mode of growth afterwards, the cup of the secondary optic vesicle is at first imperfect along its under surface, where a gap, the choroidal fissure, exists for some little time, but subsequently closes up.

The mesoblast in which the eye is imbedded gathers itself together around the optic cup into a distinct investment of which the internal layers become the choroid, the external the sclerotic. An ingrowth of this investment between the front surface of the lens and the superficial 
epiblast furnishes the body of the cornea, the epiblast itself remaining as the anterior corneal epithelium.

A portion of mesoblast, carried in from the front by the lens during its involution, gives rise to the capsule of the lens and suspensory ligament, while some mesoblast entering on the under side through the choroidal fissure becomes (in birds) the pecten, and probably also contributes to the vitreous humour.

Of the walls of the optic cup, the thinner outer (posterior) wall becomes, behind the line of the ora serrata, the pigmentepithelium of the choroid, while the thicker inner (anterior) wall supplies all the elements of the retina, including the rods and cones which grow out from it into the pigmentepithelium.

FIG. 32 .

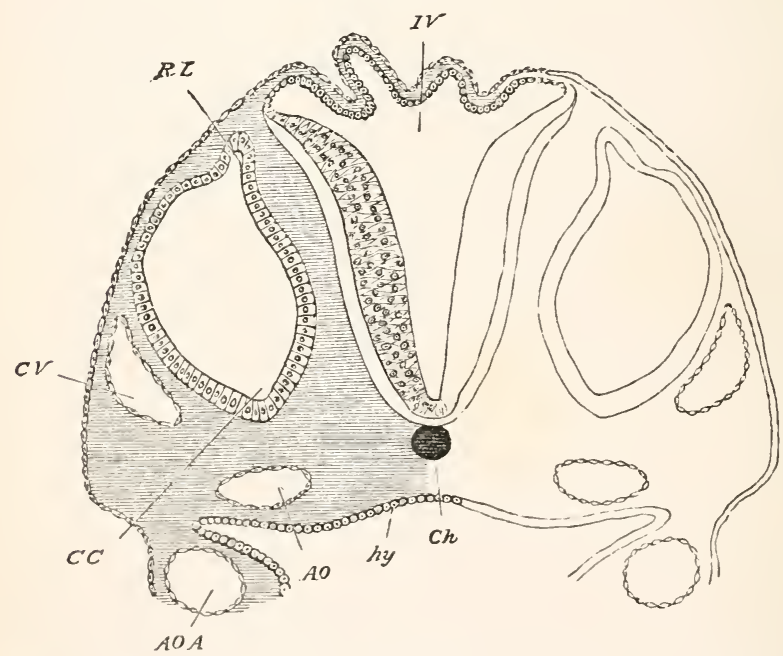

Siction through the Hind-Brain of a Chick at the exd of the Third

Day of Incubation.

IV. Fourth ventricle. The section shews the very thin roof and thicker sides of the ventricle.

Ch. Notochord-(diagrammatic shading).

$C V$. Anterior cardinal vein.

$C C$. Involuted auditory vesicle. $C C$ points to the end which will form the cochlear canal. $R L$. Recessus labyrinthi. $h y$. Hypoblast lining the alimentary canal. AO, AOA. Aorta, and aortic arch. 
In front of the line of the ora serrata, both walls of the optic cup, quite thin and wholly fused together, give rise to the pigment-epithelium of the ciliary processes and iris, the bodies of both these organs being formed from the mesoblastic investment.

12. During the second day the ear first made its appearance on either side of the hind-brain as an involution of the external epiblast, thrust down into the mass of mesoblast rapidly dereloping between the epiblast of the skin and that of the neural canal (Fig. 15, au.p.). It then had the form of a shailow pit with a widely open mouth. Before the end of the third day, its mouth closes up and all signs of the opening are obliterated. The pit thus becomes converted into a closed vesicle lined with epiblast and surrounded by mesoblast. This vesicle is the otic vesicle, whose cavity rapidly enlarges while its walls become thickened (Fig. 32, $C^{C} C$ ).

The changes by which this simple otic resicle is converted into the complicated system of parts known as the internal ear, have been much more completely worked out for mammals than for birds. We shall therefore reserve a full account of them for a later portion of this work. Meanwhile a brief statement of the main course of events in the chick may be useful; and will be most conveniently introduced here, although we shall have, in doing so, to speak of changes which do not occur till much later than the third day.

The internal ear consists essentialiy of an inner membransus labigrinth lying loosely in and only partially attached to an outer usseous labyrinth.

The membranous labyrinth (Fig. 33) consists of two parts: (1) the vestibule, with which are connected three pairs of semicircular canals, pag', fir', hor', and a long narrow hollow process, the aqueductus or recessus restibuli, and $(2)$ the ductus cochlearis, which in birds is a flask-shaped cavity slightly bent on itself. The several parts of each of these cavities freely communicate, and the two are joined together by a narrow canal, the canalis reuniens, $c r$.

The osseous labyrinth has a corresponding form, and may be similarly divided into parts: into a bony vestibule. with its bony semicircular canals and recessus vestibuli, and into a bony cochlea; but the junction between the cochlea and the bony vestibule is much wider than the membranous canalis reuniens. 
A

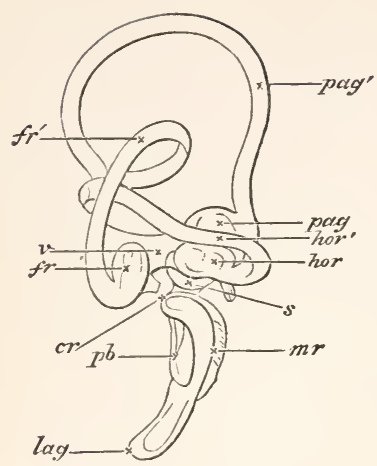

FIG. 33.

B

Two Views of the membranous Labirinth of Colomba domestica (copied froin Hasse). A from the exterior, B from the interior.

hor' horizontal semicircular canal, hor ampulla of ditto, pag' posterior vertical semicircular canal, pay ampulla of ditto, $f r^{\prime}$ anterior vertical semicircular canal, $f r$ ampulla of ditto, $u$ utriculus, $r u$ recessus utriculi, $v$ the connecting tube between the ampulla of the anterior vertical semicircular canal and the utriculus, de ductus endolymphaticus (recessus vestibuli), $s$ sacculus hemisphericus (this is smaller in birds than in any other vertebrate), $c r$ canalis reuniens, lag lagena (the dilated extremity of the cochlea), $m r$ membrane of Reissner, which forms the boundary between the scala vestibuli and scala media, $p l$ Basilar membrane, which forms the boundary between the scala tympani and the scala media.

The cochlea of a bird consists ( $\mathrm{I}$ ) of a scala vestibuli with a very small lumen, which opens at one end into the perilymphatic cavity of the vestibule, and at the other into the lagena (the dilated extremity of the cochlea corresponding with the cupola of mammals); (2l of a scala tympani, also opening into the lagena at one end, and into the foramen rotundum at the other; $(3)$ of a scala media ending blindly at one end, but in communication with the membranous vestibule at the other, through the membranous canalis reuniens $(\mathrm{cr})$.

As in mammals, the cavity of the osseous cochlea is divided lengthways by the ductus cochlearis into a scala tympani ending in a foramen rotundum, and a scala vestibuli ending in the cavity of the osseous vestibule, which in its turn is connected with the foramen ovale.

The auditory nerve, piercing the osseous labyrinth in various points, is distributed in the walls of the membranous labyrinth.

All these complicated structures are derived from the simple primary otic vesicle by changes in its form and lifferentiation of its walls. All the epiblast of the vesicle 
goes to form the epithelium of the membranous labyrinth, whose curiously twisted cavity filled with endolymph represents the original cavity which was first open to the surface but subsequently covered in. The corium of the membranous labyrinth, and all the tissues of the osseous labyrinth,

Fig. 34.

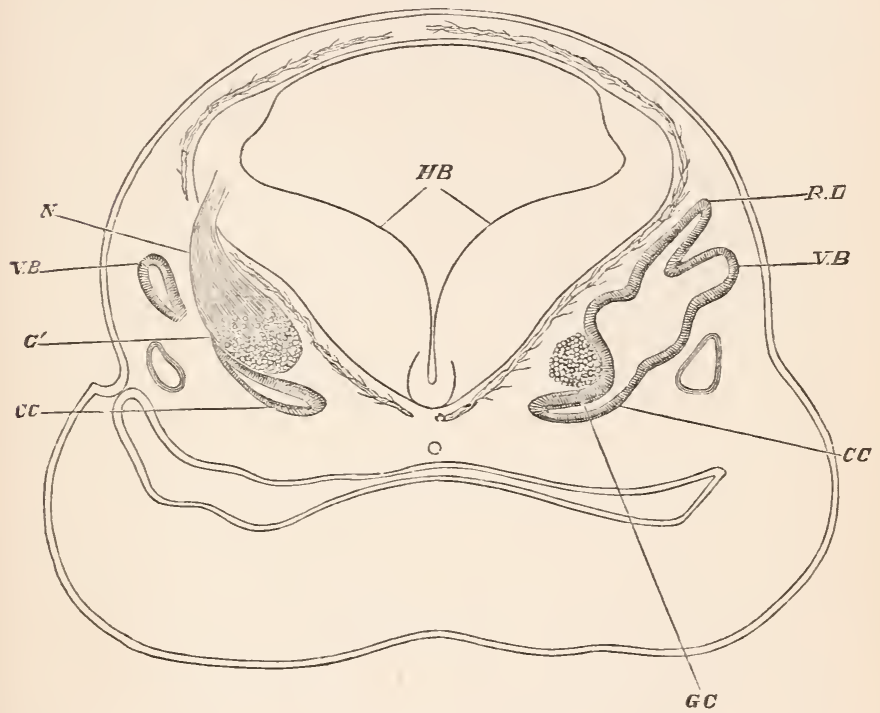

Transverse Section of the Head of a Fetal Sheep (i6 ym. in length) in the REgion of the HiNd Brain. (Copied from Boettcher, Entwickelung und Bau des Gehörlabyrinths.)

This figure, together with Figs. 33 and 34 , though referring to mammalian structures, are introduced here, in order to illustrate the account given in the text.

$H . B$. the hind brain, the lines of reference starting on both sides from the greatly thickened side walls.

The section is somewhat oblique, hence while on the right side the connections of the recessus vestibuli, R.L., and of the commencing vertical semicircular canal I.B. and of the ductus cochlearis C.C., with the cavity of the primary otic vesicle are well seen, on the left side, only the extreme end of the ductus cochlearis C.C. and of the semicircular canal V.B. are shewn. In the same way the cavity of the throat appears from the obliquity of the section to be one-sided.

Lying close to the inner side of the otic vesicle is seen the cochlear ganglion G.C. ; on the left side the auditory nerve $G$ and its connection $N$ with the hind-brain are also shewn.

Below the otic resicle on either side lies the cardinal vein. 
are developed out of the mesoblastic investment of the vesicle, the (lymphatic) space between the osseous and membranous labyrinths being hollowed out of the mesoblast, and becoming filled with perilymph as it is formed. The chief stages are as follows:-

The form of the vesicle as seen in transverse sections of the head from being oval becomes somewhat triangular with the apex directed downwards (i.e. towards the ventral surface of the body). (Fig. 32.)

The apex continues to develope until it becomes a somewhat pointed process directed inwards (Fig. 34, CC), and lying. somewhat in front (nearer the face than) the rest of the vesicle,

FIG. 35.

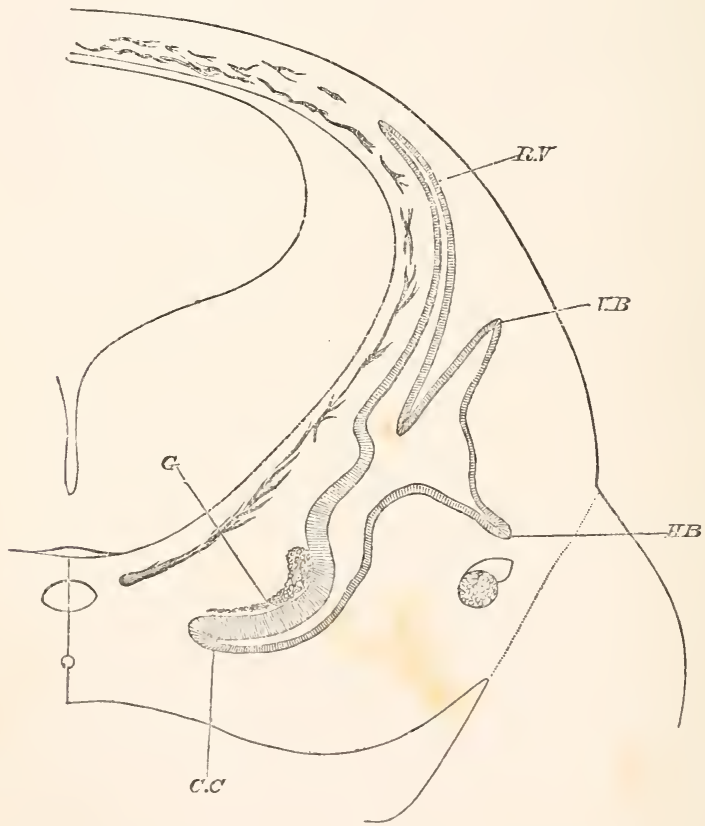

Section of the Head of a Fetal Shegr (zo mis. in levgth). (Copied from Boettcher.)

R. V. Recessus Vestibuli. V.B. Vertical semicircular canal. II.B. Horizontal semicircular canal. C.C. Cochlear canal. G. Cochlear ganglion. 
from which it soon becomes definitely marked off by a constriction as the ductus cochlearis (Fig. 3.5, C.C.), leaving the rest of the vesicle to form the vestibule and its appendages. About the same time that the ductus cochlearis is developing the upper and outer corner of the triangular vestibule grows out upwards and backwards, as a long hollow process, the recessus or aqueductus vestibuli (Fig. 35, $R V, 36, R V$ ), marked where it leaves the vestibule by a more or less prominent constriction. The upper end of this is the remnant of the primitive opening of the auditory involution.

On the remaining surface of the vestibule two hollow protuberances are visible (Fig. 34, V.B.), the rudiments of the two vertical semicircular canals. Below this, a little later on, a similar bulging starts to form the horizontal semicircular canal (Fig. 35, H.B.). Each protuberance grows out, becomes flattened, and while remaining attached to the restibule at its two ends becomes separated from it in the middle, and is thus converted into a single tube opening at both ends (one of which subsequently dilates into an ampulla) into the cavity of the vestibule.

Near the junction of the ductus cochlearis with the vestibule, two constrictions give rise to a prominence lying between them, and known as the sacculus hemisphericus. This, though very conspicuous in mammals (Fig. $36, S . R$.), is very slightly marked in birds (Fig. 33, s). The rest of the vestibule remains as the utriculus. The progressive constriction at the base of the ductus cochlearis gives rise to the canalis reuniens. Thus, by an unequal growth resulting in these protuberances and constrictions, the originally oval sac is modelled into the membranous labyrinth.

While the vesicle is thus increasing in size and changing in form, a large quantity of mesoblast is gathered round it. The outer portion of this mesoblastic investment becoming dense is converted into cartilage, while the internal portion remains as undifferentiated tissue. Later on, the most internal layers of this undifferentiated tissue are converted into the membranous coat (corium) which supports the epithelium of the membranous labyrinth; while the remainder is liquefied or absorbed at the same time that the cartilaginous envelope becomes ossified, and so gives rise to the cavity of the osseous labyrinth with its contained peri- 
Fig. 36 .

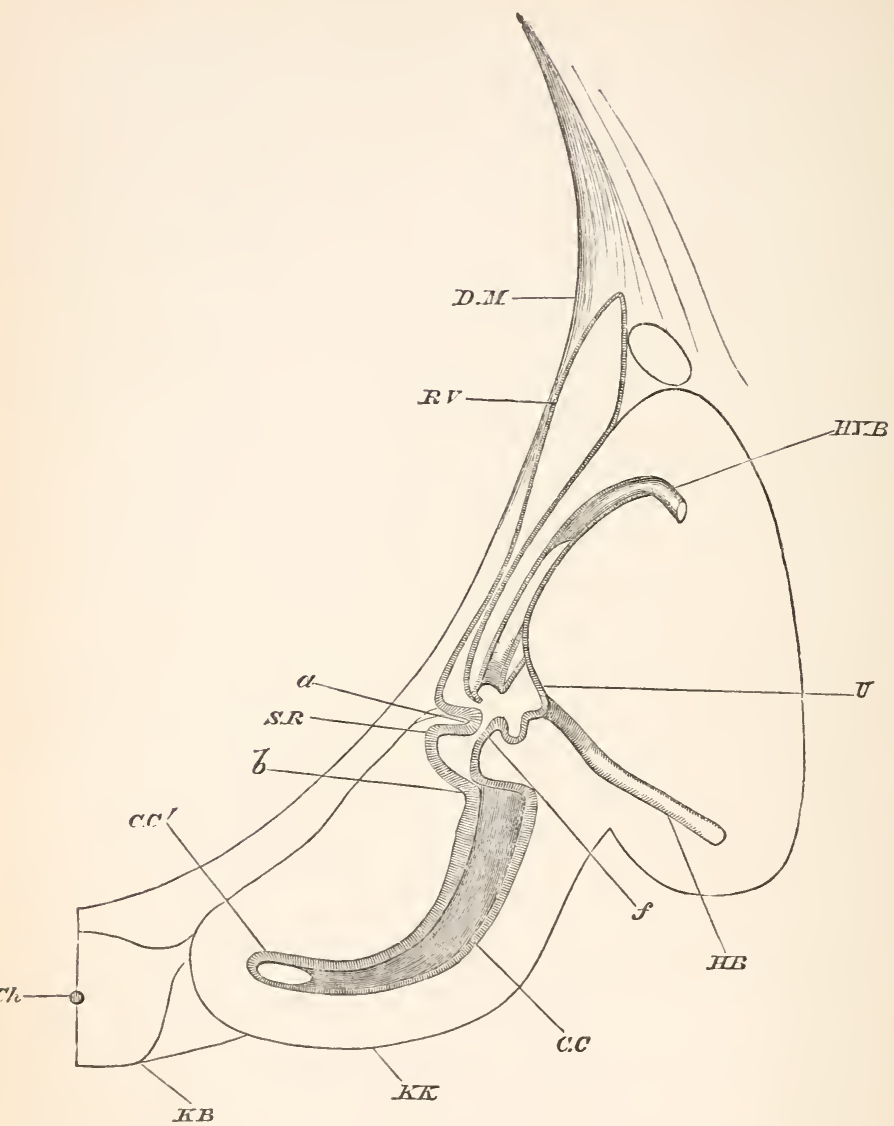

Section throdgh the Internal Ear of an Embryonic Sheep (28 mm. IN LENGTH). (Copied from Boettcher.)

D.M. Dura Mater. R.V. Recessus vestibuli. II.V.B. Posterior vertical semicircular canal. U. Utriculus. H.B. Horizontal semicircular canal. $b$. Canalis reuniens. $a$. Constriction by means of which the sacculus hemisphericus S.R. is formed. $f$. Narrowed opening between sacculus hemisphericus and utriculus. C.C. Cochlea. C.C'. Lumen of cochlea. $K . K$. Cartilaginous capsule of cochlea. K.B. Investing mass. Ch. Chorda dorsalis. 
lymph. In the region of the cochlea this excavation of the mesoblast takes place along two definite tracts, and thus the two scalæ are established; but in the region of the vestibule and its appendages it is more irregular, resulting in one common cavity interrupted at various points by bridles of connective tissue passing from the osseous to the membranous labyrinth.

Further details, especially concerning the histological changes, we propose to reserve for a later part of this work. Meanwhile we may remark that all the minute auditory apparatus, the hair-cells, and their various adjuncts, appear to be of a distinctly epithelial nature and of epiblastic origin. In the bird, as is well known, there are no rods of Corti; but even these structures seem in the mammal to be similarly epiblastic.

It will be seen then that the ear, while resembling the eye in so far as the peculiar structures in which the sensory nerve in each case terminates are formed of involuted epiblast, differs from it inasmuch as it arises by an independent involution of the superficial epiblast, whereas the eye is a constricted portion of the general involution which gives rise to the central nervous system. Still greater is the distinction between the optic and auditory nerves. The optic nerve is, as we have seen, the consolidated stalk of the optic vesicle, and therefore is of purely epiblastic origin. The auditory nerve, on the contrary, as we shall see, arises in and is formed out of the mesoblast, lying by the side of the otic vesicle. It with its ganglion may readily be recognised in sections as quite independent, both of the otic vesicle and the hindbrain, though subsequently coming into connection with each of them. The growth of the-auditory nerve into the hind-brain is shewn in Fig. $34, N$; the union of the nervefibres with the epithelial structures of the membranous labyrinth takes place at a later date.

13. At the under surface of each of the vesicles of the cerebral hemispheres there appears towards the end of the third day a small somewhat elongated vesicle, the olfactory vesicle, which is the rudiment of the olfactory nerve or bulb. Over each olfactory vesicle the external epiblast which covers them grows inwards to form a shallow depression with a thickened border. These depressions are the nasal pits (Fig. 
$\left.37, N^{\top}\right)$. Like the lens and the labyrinth of the ear, they are FiG. 37.

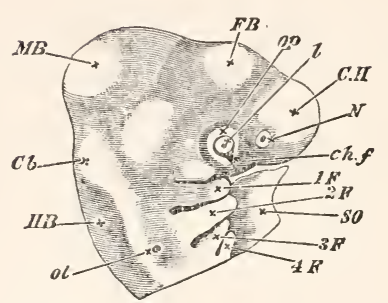

Head of an Embryo Chick of the Third Day viewed sideways as aN OPAqUE OBJECT. (Chromic acid preparation.)

C.H. Cerebral hemispheres. F.B. Vesicle of third ventricle. M.B. Mid-brain. Cb. Cerebellum. H.B. Medulla oblongata.

$\Lambda$. Nasal pit. ot. otic vesicle in the stage of a pit with the opening not yet closed up. op. Optic vesicle, with $l$. lens and $c h . f$. choroidal fissure. The small dot in the centre of the lens indicates the remnant of its external opening. The superficial epiblast moulds itself to the form of the optic vesicle and the lens; hence the choroirlal fissure, though formed entirely underneath the superficial epiblast, is distinctly visible from the outside.

I $F$. The first visceral fold; above it is seen a slight indication of the superior maxillary process.

$2,3,4 F$. Second, third and fourth visceral folds, with the visceral clefts between them.

SO. Portion of the somatopleure springing from between the ends of the visceral folds.

formed from the external epiblast; unlike them they are never closed up. At first they have no distinct connection with their respective olfactory vesicles, and their openings are independent and separate, there being as yet no actual mouth to connect them with each other.

14. It must be borne in mind that especially in the early stages of development, owing to the very unequal growth of lifferent parts, the relative position of the various structures is continually shifting. 'This is very well seen in the instance of the heart. At its first appearance, the heart is lodged immediately beneath the extreme front of the alimentary canal so far forwards as to underlie that portion of the medullary canal which will form the brain. It is, in fact, at that epoch a part of the head. From that early position it gradually recedes farther and farther backward, until, at the end of the third day, a considerable interval is 
observed between it and the actual head. In other words, a distinct neck has been formed, in which most important changes take place.

The neck is distinguished from the trunk in which the heart now lies by the important feature that in it there is no cleavage of the mesoblast into somatopleure and splanchnopleure, and consequently no pleuroperitoneal cavity. In passing from the exterior into the alimentary canal, the three layers of the blastoderm are successively traversed without any breach of continuity save such as is caused by the cavities of the blood-vessels. In this neck, so constituted, there appear on the third day certain fissures or clefts, the visceral or branchial clefts. These are real clefts or slits passing right through the walls of the throat, and are placed in series on either side across the axis of the alimentary canal, lying not quite at right angles to that axis and parallel to each other, but converging somewhat to the middle of the throat in front (Fig. 37). Viewed from the outside in either fresh or preserved embryos they are not very distinctly seen to be clefts; but when they are seen from within after laying open the throat, their characters as elongated oval slits can easily be recognised.

Four in number on either side, the most anterior is the first to be formed, the other three following in succession. Their formation takes place from within outwards. The hypoblast and mesoblast are first absorbed along the line of the future cleft, then the epiblast is broken through, and the hypoblast, which is carried outwards as a lining to the slit, forms a junction with the epiblast at the outside of the throat.

No sooner has a cleft been formed than its upper border (i.e. the border nearer the head) becomes raised into a thick lip or fold, the visceral or branchial fold. Each cleft has its own fold on its upper border, and in addition the lower border of the fourth or last visceral cleft is raised into a similar fold. There are thus five visceral folds to four visceral clefts (Fig. 37). The last two folds however, and especially the last, are not nearly so thick and prominent as the other three, the second being the broadest and most conspicuous of all. The first fold meets, or nearly meets its fellow in the middle line in front, but the second falls short 
of reaching the middle line, and the third, fourth and fifth do so in an increasing degree. Thus in front views of the neck a triangular space with its apex directed towards the head is observed between the ends of the several folds.

Into this space the pleuroperitoneal cavity extends, the somatopleure separating from the splanchnopleure along the ends of the folds; and it is here that the aorta plunges into the mesoblast of the body.

Of the history of these most important visceral folds and clefts we shall speak in detail hereafter; meanwhile we may say that in the chick and higher vertebrates the first three pairs of folds are those which call for most notice.

The first fold on either side, increasing rapidly in size and prominence, does not, like the others, remain single, but sends off in the course of the third day a branch or bud-like process from its upper edge. This branch, starting from near the outer beginning of the fold, runs forwards and upwards, tending to meet the corresponding branch from the fold on the other side, at a point in the middle line nearer the front of the head than the junction of the main folds. The two branches do not quite meet, being separated by a median process, which at the same time grows down from the extreme front of the head, and against which they abut. Between the main folds, which are directed somewhat downwards and the branches which slant upwards, a somewhat lozenge-shaped space is developed which, as the folds become more and more prominent, grows deeper and deeper. The main folds are the rudiments of the inferior maxille, the branches those of the superior maxilice, the lozengeshaped cavity between them is the cavity of the mouth, and the descending process which helps to complete the upper margin of this cavity is called, from the parts which will be formed out of it, the fronto-nasal process.

Already on the second day the under wall of the front end of the alimentary canal began to become thin, while over it the epiblast was pushed in so as to form a depression. The maxillary folds convert this depression into a deep pit, whose bottom is not as yet perforated, the opening into the alimentary canal being made later on.

The two succeeding pairs of visceral folds are transformed into parts which will be best considered in connection with the development of the skull. The 
last two disappear in the chick without giving rise to any permanent structures.

The first visceral cleft remains permanently open, but is drawn out by the growth of surrounding parts into a long tube, subsequently divided into the meatus auditorius and the Eustachian tube. The other visceral cletts are obliterated.

15. By the end of the second day three pairs of aortic arches had been established in connection with the heart. When the visceral folds and clefts are formed, a definite arrangement between them and the aortic arches is alway's

Fig. 38 .

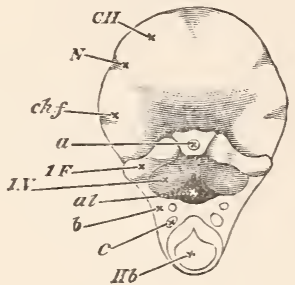

The same Head as shewn in Fig. 37, seen froy the Front.

The neck has been cut across between the first and second visceral folds, the incision being carried through the first visceral cleft. In the cut surface $b$ are seen the sections of the hind brain $H b$., and of blood-vessels $c$.

I $F$. The first visceral fold; between the ends of the fold is seen a section of the somatopleure at its extreme forward limit; in it lies the aorta $a$. Below the folds is the cavity of the throat $a l$, and $I . V$ is placed in the first visceral cleft.

C.H. Cerebral hemispheres. $N$. Nasal pit. ch.f. groove indicating the choroidal fissure.

observed. The first visceral cleft runs between the first and second aortic arches. Consequently the first aortic arch runs in the first visceral fold and the second in the second. In the same way, the second visceral cleft lies between the second and third aortic arches, the third aortic arch running in the third visceral fold. Each aortic arch runs in the thickened mesoblast of the corresponding fold.

Arrived at the upper surface of the alimentary canal, these arches unite at acute angles to form a common trunk, the dorsal aorta (Fig. $39 \mathrm{~A}$, A. O), which runs along the back immediately under the notochord. The length of this common single trunk is not great, as it soon divides into two main branches 
FiG. 39. A.

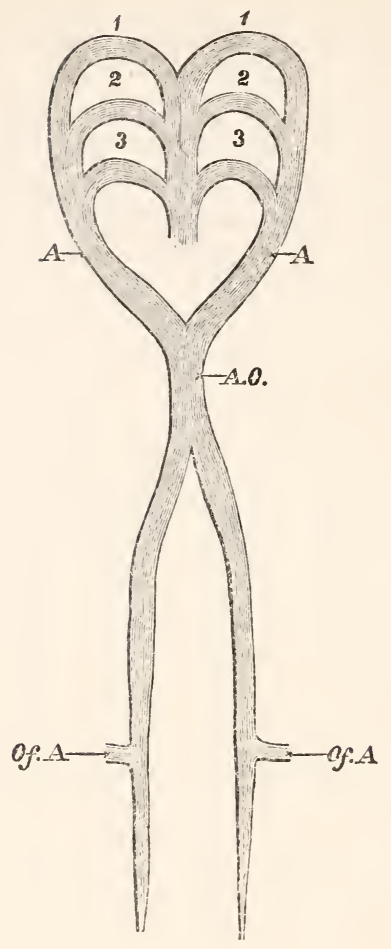

Diagray of the Arterial Circulation on the Third Day.

I, 2, 3. The first three pairs of aortic arches. $A$. The vessel formed by the junction of the three pairs of arches. A.O. Dorsal aorta formed by the junction of the two branches $A$ and $A$; it quickly divides again into two branches which pass down one on each side of the notochord. From each of these, not far from its termination, is given off a large branch Of.A., the omphalo-mesaraic artery.

(the future common iliacs), each of which, after giving off the large omphalo-mesaraic artery, Of.A., pursues its course with diminished calibre to the tail, where it is finally lost in the capillaries of that part.

16. The heart is now completely doubled up on itself. Its mode of curvature is apparently somewhat complicated. 
Starting from the point of junction of the omphalo-mesaraic veins (Fig. 2t, $H t$ ), there is first a slight curvature towards the left; this is followed by a turn to the right, and then the heart is completely bent on itself, so that afterwards it pursues a course directed from behind quite straight forwards (except perhaps for a little inclination to the left) to the point where the aortic arches branch off. In this way the end of the bulbus arteriosus comes to lie just underneath (or in front of according to the position of the embryo) that part which has already been marked off by the lateral bulgings as the auricular portion.

That part of the heart which is turned to the right, including the point of doubling up, is the ventricular portion, and is even at this stage separated from the auricular portion by a slight neck. This external constriction corresponds to an internal narrowing of the lumen of the heart, and marks the position of the future canalis auricularis.

The ventricular portion is, on the other hand, likewise separated by a fainter constriction from the anterior continuation of the heart which forms the bulbus arteriosus. The projecting part where the doubling takes place is at this stage still quite round; we shall see that later on it becomes pointed and forms the apex of the heart.

The whole venous portion of the heart (if we may so speak, though of course at this stage blood of the same quality passes right along the whole cardiac canal) lies in a plane which is more dorsal than the arterial portion. The point at which the venous roots of the heart, i.e. the two omphalo-mesaraic trunks unite into a single canal, is on this day carried farther and farther away from the heart itself. By the end of the day there is a considerable distance between the auricular portion of the actual heart and the point where the venous roots separate each to pursue its course along the splanchnopleure-fold of its own side. This distance is traversed by a single venous trunk, of which the portion close to the auricles is called the sinus venosus, and the more distant the ductus venosus. We shall give to the whole trunk the name used by the older observers, the neatus venosus.

Small arteries to various parts of the body are now being given off by the aorta and its branches. The capil- 
Fig. 39. B.

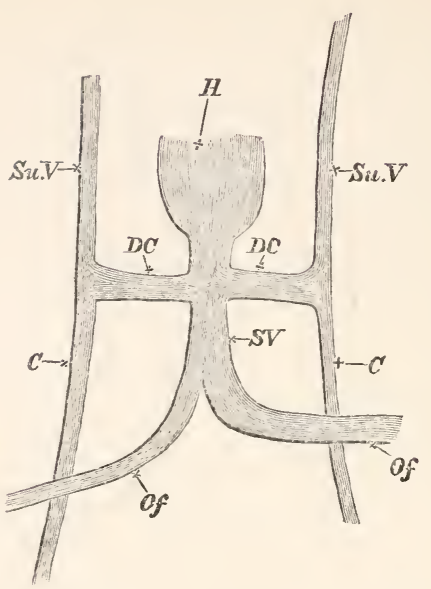

Diagrair of the Venous Crrculation on the Third Day.

II. Heart. D.C. Ductus Cuvieri. S.V. Meatus venosus. Su.V. Superior vertebral or anterior cardinal vein. $C$. Inferior or posterior cardinal vein. Of. Omphalo-mesaraic vein.

laries in which these end are gathered into veins which unite to form two main trunks on either side, the cardinal veins, anterior and posterior (Fig. 23, Fig. $39 \mathrm{~B}, S_{u} . V$. and $C^{\prime}$.), which run parallel to the long axis of the body in the upper part of the mesoblast, a little external to the protovertebræ. These veins, which have not by the third day attained to any great importance, unite opposite to the heart, on each side, into a short common trunk at right angles to themselves. The two short trunks thus formed, which bear the name of Ductus Cuvieri (Fig. 23, Fig. 39 B, D.C.), runuing transversely straight inwards towards the middle line fall into the sinus venosus.

The blood-vessels in the body of the embryo take their origin from the mesoblast exclusively, and their formation is probably precisely similar to that of the vessels in the vascular area, branches being given out from or brought into connection with the aorta and omphalo-mesaraic veius, in the same way as branches were described as springing from or meeting the earliest formed vascular channels.

His, carrying out the views we have already referred to, believed that parablastic elements grew inwards along the omphalo-mesaraic trunks, through the length of the heart and so onwards into the aorta and all its branches, until 
the whole archiblastic framework of the embryo became riddled by a network of parablast. From these parablastic elements he considered there arose not only the epithelium (endothelium) of the blood-vessels and lymphatic spaces, but also all the connective-tissue elements of the body, the archiblast being represented in the vessels of the adult body by the muscular fibres alone.

17. As we stated above (p. 87), the folding in of the splanchnopleure to form the alimentary canal is proceeding with great rapidity, the tail-fold as well as the head-fold contributing largely to the result.

The formation of the tail-fold is very similar to that of the head-fold. At the extreme hind end of the embryo, at the tip of the tail (Fig. $40, t$ ), there is no cleavage of the mesoblast, and therefore neither somatopleure nor splanchnopleure. The tail is a solid, somewhat curved, blunt cone of mesoblast, immediately coated with the superficial epiblast

\section{Fig. 40.}

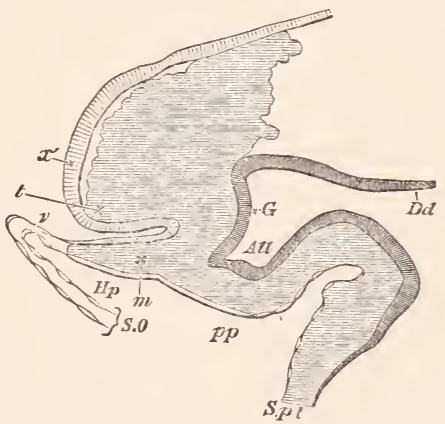

Section of the Tail-exd of an Embrio (Chick) of the Third Day. (From Dobrynin.)

$t$. the tail. So. somatopleure. $S p l$. splanchnopleure. $p p$. pleuroperitoneal space. The letters $G$ and $D d$ are placed within the alimentary canal: a more detailed explanation of the figure is given under Fig. 50.

except at the upper surface (corresponding to the back of the embryo), where lies the pointed termination of the neural tube. At some little distance forwards, towards the head, the cleavage of the mesoblast begins, and the somatopleure diverges from the splanchnopleure, the latter, as in the headfold, being folded in to a greater extent than the former. Except for the absence of the cephalic enlargement of the neural tube, the presence of distinct protorertebræ up to nearly the 
FIG. 4 I.

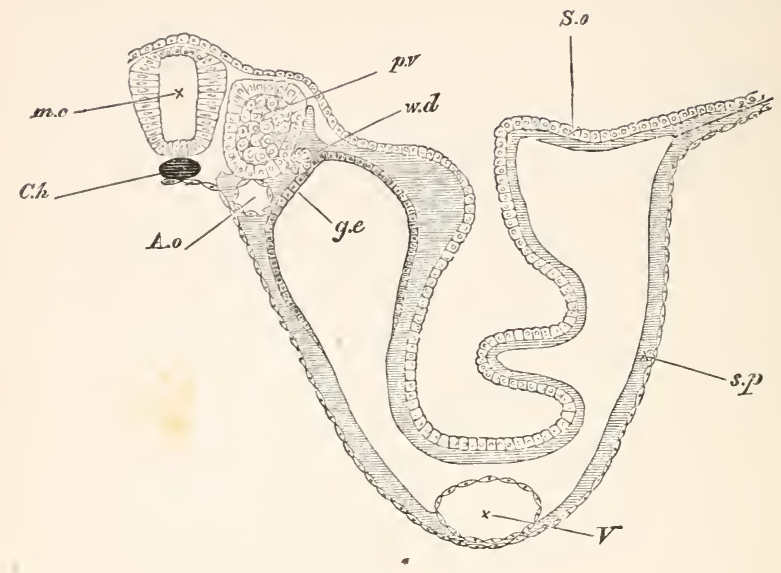

\section{Section through the Dorsal Region of an Embryo at the comingenment of the Third DaY. .}

H. C. medullary canal. Ch. notochord. $p . v$. protovertebra composed of an investment of columnar cells enclosing a central nucleus of rounded cells. $w$. d. Wolffian duct, which has commenced to travel down from the dorsal surface of the mesoblast. A. o. dorsal aorta of right side. g.e. germinal epithelium ; an epithelium of columnar cells lining the upper end of the pleuroperitoneal cavity, and which is related to the formation of Muiller's duct and of the ovary. S. o. somatopleure. s. p. splanchnopleure.

The splanchnopleure is very little folded in, the embryonic sac being in this middle region widely open to the yolk below. The somatopleure is much more folded in. At a little distance outside the protovertebra is a ridge with thickened mesoblast, the Woiffian ridge, marking the line along which the limbs will be developed. Beyond this ridge the somatopleure suddenly descends to form the hody-wall (of the abdomen); it then ascends, and after a fold, probably due to the action of the chromic acid, forms an angular projection at about the level of the protovertebra. This projection is the lateral fold of the amnion, the section having been taken just at that point between the head and tail where the amnion is, on the third day, least developed.

Beyond the amniotic fold the somatopleure and splanchnopleure come into apposition, and still farther out completely coalesce. It will be observed that the pleuroperitoneal space already reaches laterally far beyond the limits of the embryo itself.

In the splanchnopleure is seen at $V$ a section of a large branch of the omphalo-mesaraic trunk.

The shating of the general mesoblast is diagrammatic.

The width of the cavity of the neural tube is unusually great. 
actual termination of the tail, and certain features connected with the development of the allantois, of which we shall speak presently, the tail is a counterpart of the head.

So rapid is the closure of the splanchnopleure both in front and behind, that two of the three parts into which the digestive tract may be divided, are brought, on this day, to the condition of complete tubes.

The first division, extending from the mouth to the duodenum, is completely folded in by the end of the day; so likewise is the third division comprising the large intestine and the cloaca. The middle division, corresponding to the future small intestine, still remains quite open to the yolksac below.

The attachment of the newly formed alimentary canal to the body above is at first very broad, and only a thin stratum of mesoblast separates the hypoblast of the canal from the notochord and protovertebræ; even that may be absent under the notochord (Fig. 41). During the third day, however, along such portions of the canal as have become regularly enclosed, i.e. the hinder division and in the posterior moiety of the anterior division, the mesoblastic attachment becomes narrower and (in a vertical direction) longer, the canal appearing to be drawn more downwards (or according to the position of the embryo forwards), away from the vertebral column.

In what may be regarded as the pleural division of the general pleuroperitoneal space, along that part of the alimentary canal which will form the osophagus, this withdrawal is very slight (compare Fig. 32), but it is very marked in the peritoneal space. Here such parts of the digestive canal as are formed come to be suspended from the body above by a narrow flattened band of mesoblastic tissue which reaches from the neighbourhoor of the notochord, and becomes continuous with the mesoblastic coating which wraps round the hypoblast of the canal. This flattened band is the mesentery, shewn commencing in Fig. 44, and much more advanced in Fig. $47, M$. It is covered on either side by a layer of flat cells, while its interior is composed of indifferent tissue.

The front division of the digestive tract consists of three parts. The most anterior part, the cesophagus, still ends blindly in front, and reaches back as far as the level of the 
lind end of the heart. Its transverse section, which up to the end of the second day was somewhat crescent-shaped, with the convexity downwards, becomes on this day more nearly circular. Close to its hinder limit, the lungs (Fig. 42, $l g$ ), of whose formation we shall speak directly, take their origin.

FIG. 42 .

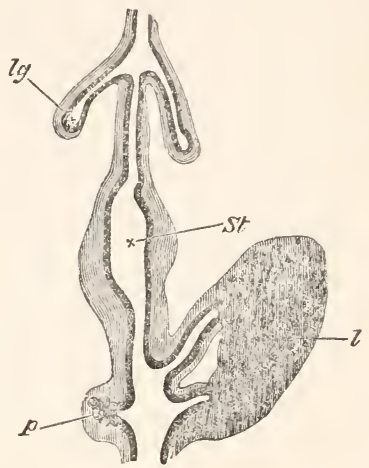

Diagray of a portion of the Digestive Tract of a Chick upon the Fourth Day. (Copied from Götte.)

The black inner line represents the hypoblast, the outer shading the mesoblast. lf. lung-diverticulum with expanded termination, forming the primary lung-vesicle. St. stomach. $l$. two hepatic diverticula with their terminations united by solid rows of hypoblast cells. $p$. diverticulum of the pancreas with the vesicular diverticula coming from it.

The portion of the digestive canal which succeeds the œsophagus, becomes towards the close of the third day somewhat dilated (Fig. 42, St) ; the region of the stomach is thus indicated.

The hinder or pyloric end of the stomach is separated by a very small interval from the point where the complete closing in of the alimentary canal ceases, and where the splanchnopleure-folds spread out over the yolk. This short tian is nevertheless clearly marked out as the duodenum by the fact that from it, as we shall presently point out, the rudiments of ducts of the liver and pancreas are beginning to be formed. It forms the third part of the front division.

The posterior division, corresponding to the great intestine and cloaca, is from its very first formation nearly circular in 
section and of a larger bore than the cesophagus. Up to the end of the day it is still completely closed at the hinder extremity, which however is somewhat swollen to form the cloaca.

18. The lungs are in origin essentially buds or processes from the primitive œsophagus.

If the alimentary canal of the chick at the end of the third day be dissected out and laid open, there will be seen on either side of the hinder end of the œsophagus a short pouch or diverticulum enveloped in a mass of mesoblast (Fig. 42, lg.; here however they are somewhat advanced, the specimen belonging to the fourth day). These pouches are the early rudiments of the lungs. Their mode of origin is as follows.

Fig. 43.
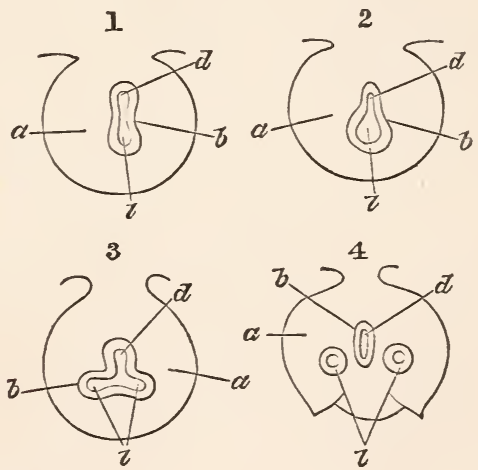

Focr diagrays illustratixg the Formation of the Lungs. (Copied from Götte.)

a. mesoblast. b. hypoblast. $d$. cavity of digestive canal. $l$. cavity of the pulmonary diverticulum.

In (I) the digestive canal has commenced to be constricted into an upper and lower canal; the former the true alimentary canal, the latter the pulmonary tube; the two tubes communicate with each other in the centre.

In (2) the lower (pulmonary) tube has become expanded.

In (3) the expanded portion of the tube has become constricted into two tubes, still communicating with each other and with the digestive canal.

In (4) these are completely separated from each other and from the digestive canal, and the mesoblast has also begun to exhibit externally changes corresponding to the internal changes which have been going on.

E. 
At a point immediately behind (or when the embryo is placed on its face, above) the heart, the cavity of the alimentary canal is compressed laterally, and at the same time constricted in the middle so that its transverse section (Fig. 43, 1) is somewhat hour-glass-shaped, and shews an upper or dorsal chamber $d$, joining on to a lower or ventral chamber $l$ by a short narrow neck. The lower chamber then becomes broader than high (Fig. 43, 2) while its under wall is raised up in a median fold, which partially divides the chamber into two lateral parts (Fig. 4:3, 3). As a result of these folds, the original simple tube becomes divided into three grooves or incomplete tubes, whose cavities all communicate with each other at the centre of the original canal: into a single tube above, which is the true œsophagus, and into two tubes below, each of which is a rudiment of a lung. The presence of these three incomplete tubes may be traced, by sections at various levels, for a certain distance along the alimentary canal, and are then lost, the canal once more returning to the condition of a simple tube.

The median fold or partition then rises up so as completely to shut off the three cavities from each other (Fig. 43, 4). The isolation commences behind and travels thence forwards, but never quite reaches the point in front where the division into three cavities begins. As a consequence the lower pulmonary tubes, though closed behind, open into the osophagus in front. In other words, by the constriction and median folds which we have described, the two short pouches or diverticula of the lungs are developed from the under (or anterior) surface of the œsophagus.

At their first origin both diverticula together with the alimentary canal itself are invested in one common rounded mass of mesoblast whose external contour bears no marks of the internal changes which are going on. By and bye, as the diverticula diverge behind from the median line, they carry the mesoblast with them (Fig. 43, 4), Then for the first time they become evident from the outside.

The subsequent history of these diverticula may with convenience be briefly lescribed here.

At first the two diverticula have separate openings into the œsophagus; but as the print at which they enter is carried further forwards (or rather as they in the process of growth are carried backwards), they unite near their base to form 
a common tube opening on the under surface of the œsophagus. This tube is the trachea.

At the end of each of the primary diverticula is a small vesicle which may be called the primary lung-vesicle. It appears ultimately to become the abdominal air-sac.

The mesoblast round the primary diverticula becomes greatly thickened. Into it secondary diverticula, still lined by hypoblast, penetrate; these again give rise to tertiary branches and thus the bronchial tubes are formed. At right angles to the finest of these the arborescent branches so characteristic of the avian lung are given off. The whole pulmonary structure is therefore the result of the growth by budding of a system of branched hypoblastic tubes in the midst of a Inass of mesoblastic tissue, the hypoblastic elements giving rise to the epithelium of the tubes and the mesoblast providing the elastic, muscular, cartilaginous, connective and other tissues of the tracheal and bronchial walls.

The air-sacs are primarily the dilated ends of the primitive diverticula or of their main branches. At first there are three air-sacs on each side: one abdominal (the end of the primitive diverticulum), another thoracic, and a third extra-thoracic. An additional thoracic, and an additional extra=thoracic, making in all five air-sacs on each side, appear at a later period.

The pulmonary blood-vessels penetrate into the mesoblast surrounding the bronchial tubes on about the i 2 th day.

19. The liver is the first formed chylopoietic appendage of the digestive canal, and arises between the 5.5th and 60th hour as a couple of diverticula one from either side of the duodenum immediately behind the stomach (Fig. 42, l.). These diverticula, though composed of both hypoblast and mesoblast, are, according to Götte, in the first instance solid, and only subsequently become hollow. The right one is, in all cases, from the first longer, but of smaller diameter than the left. Situated a little behind the heart, they embrace between them the meatus venosus or united trunk of the omphalo-mesaraic veins, which as it passes between them exhibits numerous small bulgings. As yet the vein and the diverticula, though in close contact, are not connected.

Towards the end of the third day there may be observed in the greatly thickened mesoblastic investment of either diverticulum a number of cylindrical solid aggregations of cells connected with, and apparently outgrowths from, the hypoblast of the diverticulum. These cylinders rapidly increase in number, apparently by division, their somewhat swollen peripheral extremities come into contact and unite. And thus, about the ninetieth hour, a sort of network of solid thick strings of hypoblastic cells is formed, the mesoblast in the meshes of the network becoming at the same time largely c:nverted into blood-vessels. In addition to this network of solid hypoblastic cylinders, the diverticula also send out 
hollow processes, lined with hypoblast. Each diverticulum becomes in this way surrounded by a thick mass composed partly of solid cylinders, and to a less extent of hollow processes, continuous with the cylinders on the one hand, and the main diverticulum on the other, all knit together with commencing blood-vessels and unchanged mesoblastic tissue. Between the two masses runs the meatus venosus, with the bulgings on which, referred to above, the blood-vessels in each mass are connected.

Early on the fourth day each mass sends out underneath the meatus venosus a solid projection of hypoblastic cylinclers towards its fellow, that from the left side being much the longest. The two projections unite and form a long solud wedge, which passes obliquely down from the right (or from the embryo lying on its left side, the upper) mass to the left (or lower) one. In this new wedge may be seen the same arrangement of a network of hypoblastic cylinders filled in with vascular mesoblast as in the rest of the liver. The two original diverticula with their investing masses represent respectively the right and left lobes of the liver, and the wedge-like bridge connecting them is the middle lobe.

During the fourth and fifth days the growth of the solid, lobed liver thus formed is very considerable; the hypoblastic cylinders multiply rapidly, and the network formed by them becomes very close, the meshes containing little more than blood-vessels. The hollow processes of the diverticula also ramify widely, each branch being composed of a lining of hypoblast enveloped in a coating of spindle-shaped mesoblastic cells. The blood-vessels are in direct connection with the meatus venosus-have become, in fact, branches of it. It may soon be observed, that in those vessels which are connected with the posterior part of the liver (Fig. 53), the stream of blood is directed from the meatus venosus into the network of the liver. In those connected with the anterior part the reverse is the case; here the blood flows from the liver into the meatus venosus. The thick network of solid cylinders represents the hepatic parenchyma of the adult liver, while the hollow processes of the diverticula are the rudiments of the biliary ducts.

The exact morphological significance of these anastomosing cylinders, and 
the manner of their ultimate metamorphosis into the ordinary hepatic tissue, is not as yet quite clear. If we suppose each solid cylinder to represent a duct with its lumen almost, but not quite, completely obliterated, we should gain a view agreeing very closely with that put forward by Hering on the structure of the adult liver.

During the fifth day, a special sac or pouch is developed from the right primary diverticulum. This pouch, consisting of an inner coat of hypoblast, and an outer of mesoblast, is the rudiment of the gall-bladder.

20. About the middle of the third day, the pancreas (Fig. $42, p$.) also appears, but its exact mode of origin is still somewhat doubtful.

According to Götte (Beit. z. entwick. des Darmcanals des Hühnchens) it commences as a thickening of both the hypoblast and mesoblast of a portion of the wall of the digestive canal on the same level as the left diverticulum of the liver. The hypoblast in the centre of this thickening becomes hollow, forming a cavity connected with the inside of the digestive canal by a narrow opening. Around this cavity processes of hypoblast are seen on the fourth day stretching into the surrounding mesoblast. These processes, which are at first solid but afterwards become hollow and ultimately branched, are in the early stages so completely covered up by mesoblast that they are not visible on the exterior. The primary cavity elongates into the duct, the hollow processes representing its branches. On the sixth day a new similar outgrowth takes place between the primary one and the stomach. This, which ultimately coalesces with its predecessor, gives rise to the second duct, and forms a considerable part of the adult pancreas. A third duct is formed at a much later period. According to this view, with which those put forward by Kölliker and Remak in the main agree, the so-called 'secreting' cells of the pancreas as well as the epithelial liring of the ducts are derived from hypoblast. Schenk (Die Bauchspeicheldriise des Embryos. Anat. u. Physiol. Untersuchung. Wien. S. I.) however is of opinion that the former originate in a transformation of the mesoblast, the hypoblast giving rise to the epithelium of the ducts only.

Shortly after the first appearance of the pancreas, the spleen appears as a thickening of the mesentery of the stomach (mesogastricum) and is therefore entirely a mesoblastic structure.

Its development has been recently investigated by Peremeschko (Sitz. der k. Akad. in Wien, Bd. 56, ז867) and by W. Mïller (Stricker's Histology).

According to these investigators, the mass of mesoblast which forms the spleen becomes early separated by a groove on the one side from the pancreas and on the other from the mesentery. Some of its cells become elongated, and send out processes which, uniting with like processes from other cells, form the trabecular system. From the remainder of the tissue are derived the cells of the spleen pulp, which frequently contain more than one nucleus. Especial accumulations of these take place at a later period to form the so-called MIalpighian corpuscles of the spleen.

21. The thyroid body is also formed towards the end of the third day, in connection with the alimentary canal. 
According to Miiller (L'eber die Entacickelung der Schilddriise. Jenaische Zeitschrift, 1871) who has recently studied its development with great care, the thyroid body arises on the third day as an involution from the hy oblast of the throat opposite the point of origin of the second arterial arch. This involution becomes by the fourth day a solid mass of cells, and by the fifth ceases to be connected with the epithelium of the throat, becoming at the same time bilobel. By the seventh day it has travelled somewhat backwards, and the two lobes have completely separated from each other. By the ninth day the whole is invested by a capsule of connective tissue, which sends in septa dividing it into a number of lobes or solid masses of colls, and by the sixteenth day it is a paired body composed of a number of follicles, each with a 'membrana propria,' and separated from each other by septa of connective tissue, much as in the adult.

22. Coincidently with the appearance of these several rudiments of important organs in the more or less modified splanchnopleure-folds, the solid trunk of the embryo is undergoing marked changes.

When we compare a transverse section taken through say the middle of the trunk at the end of the third day (Fig. 4t), with a similar one of the second day (Fig. 20), or even the commencement of the third day (Fig. 41 ), we are struck with the great increase of depth (from dorsal to ventral surface) in proportion to breadth. This is partly due to the slope of the side walls of the body having become much steeper as a direct result of the rapidly progressing folling off of the embryo from the yolk-sac. But it is also brought about by the great changes both of shape and structure which are taking place in the protovertebræ as well as by the development of a mass of tissue between the notochord and the hypoblast of the alimentary canal.

23. The protovertebra on the second day, as seen in a transverse section, Fig. 20, P.v., are somewhat quadrilateral in form but broader than high. Each at that time consists of a somewhat thick cortex of radiating rather granular columnar cells, enclosing a small kernel of spherical more transparent cells.

Remak and after him Kölliker have described the centre of the protovertebre as being simply fluid without structural elements. His explicitly denies this in the case of the protovertebra of the neck, and it seems probable that the centre is in all cases really occupied by transparent spherical cells.

Towards the end of the second and the begimning of the third day, the central cells increase rapidly in number (Fig. 41), and towards the end of the latter day (Fig. 44), as it were lift up and push out the columnar cortex above and at the outer side. In this way the portions forming the 


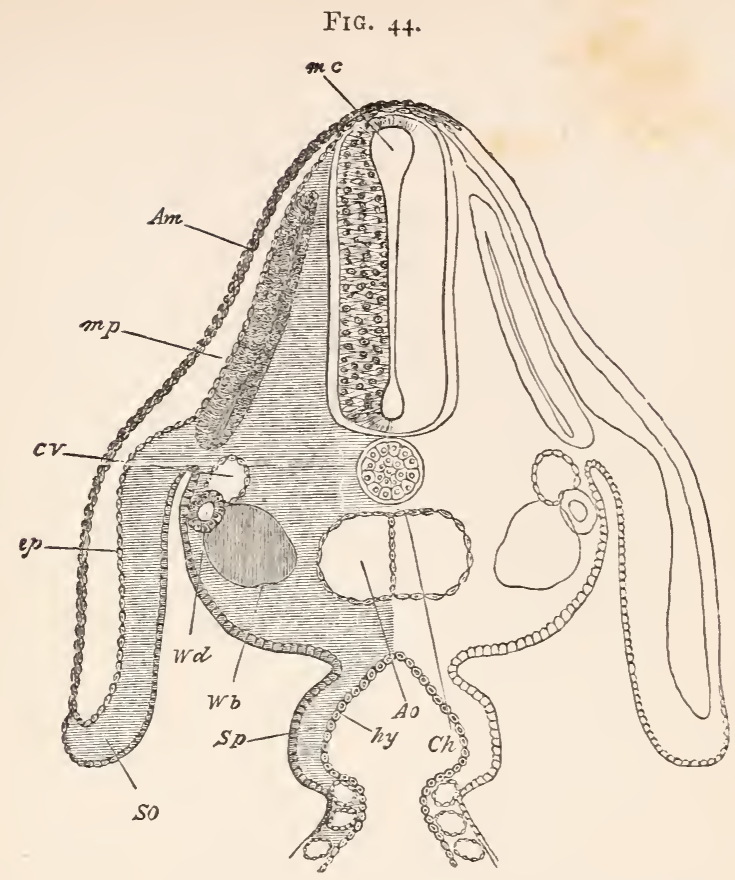

\section{Section throdgh the Dorsal Region of an Eybryo at the end of the Third DaY.}

$A m$. amnion. m. p. muscle-plate. C. V. cardinal vein. Ao. dorsal aorta. The section passes through the point which the dorsal aorta is just commencing to divide into two branches. Ch. notochord. W. $d$. Wolffian duct. W. $b$. commencing differentiation of the mesoblast cells to form the Wolffian body. $e p$. epiblast. SO. somatopleure. Sp. splanchnopleure. hy. hypoblast. The section passes through the point where the digestive canal communicates with the yolk-sac, and is consequently still open below.

This section should be compared with the section through the dorsal region of an embryo at the commencement of the third day (Fig. $+\mathrm{I}$ ). The chief differences between them arise from the great increase in the space (now filled with mesoblast-cells) between the notochord and the hypoblast. In addition to this we have in the later section the completely formed amnion, the separation of the muscle-plate from the protovertebræ, the formation of the Wolffian body, etc.

The mesoblast including the Wolffian body and the muscle-plate (m.p.) is represented in a purely diagrammatic manner. The amnion, of which only the inner limb or true amnion is represented in the figure, is seen to be composed of epiblast and a layer of mesoblast; though in contact with the body above the top of the medullary canal, it does not in any way coalesce with it, as might be concluded from the figure. 
upper and outer border of the protovertebra become separated from the rest of the cortex, the columnar cells of the latter at the same time losing their distinctive characters and ceasing to be distinguishable from the central cells. As a consequence of this the whole protovertebra, while thus increasing in breadth out of proportion to its height, becomes split up into two portions which lie one above the other. Of these the upper one, which is from the first the most Hattened and longest, follows the curvature of the body-wall and thus from being nearly horizontal comes to slope at a considerable angle. It now receives the name of muscle-plate, Fig. 4t, m.p. Ot its subsequent changes we shall have to speak in a succeeding chapter.

The remaining portion of the original protovertebra is still called protovertebra and begins to extend inwards over the neural canal above and towards the notochord below.

24. Meanwhile the breadth or rather depth of the trunk is also being increased by the development of mesoblastic cells between the notochord and hypoblast.

In a transverse section of a 45 hours' embryo a considerable mass of cells may be seen collected between the protovertebra and the point where the divergence into somatopleure and splanchnopleure begins (Fig. 20 just below W.d., also Fig. 41, the diagrammatically shaded part lying between p.v. and g.e). 'This mass of cells, which we may speak of as the intermediate cell-mass, now passes without any very sharp line of demarcation into the protovertebra itself; and as the folding in of the side wall progresses, increases in size and grows in between the notochord and the hypoblast, but does not accumulate to a sufficient extent to separate them widely until the end of the third or beginning of the fourth day.

The fusion between the intermediate cell-mass and the outer and under portions of the altered protovertebræ becomes so complete on the third day that it is almost impossible to say which of the cells in the immediate neighbourhood of the notochord are derived from the protovertebræ and which from the intermediate cell-mass. It seems probable however that the cells which form the immediate investment of the notochord really belong to the protovertebra. 
Schenk (Wien. Sitz. Bericht. I 868 ) describes all the cells which invest the hypoblast of the digestive tract, as primarily derived from the protovertebræ, with the exception of the peritoneal epithelium, which alone, he considers, is the representative of the original mesoblast of the splanchnopleure. According to this view, the muscles of the walls of the alimentary canal, and the 'hypaxial' nuscles, are derived from the original protovertebræ, quite as much as those muscles which spring out of the muscle-plate. In the absence of any satisfactory means of distinguishing the cells of the intermediate cell-mass from those of the protovertebra, this view must be considered as at least very doubtful.

2.5. In the mesoblast, which lies by the side of the hind brain, and which though not divided into protovertebræ is the prolongation forwards of the same column of mesoblast out of which in the trunk the protovertebre are formed, there appear on either side in the course of the third day a series of four small opaque masses, somewhat pearshaped with the stalk directed away from the middle line. These are the rudiments of four cranial nerres, of which two lie in front of and two behind the auditory vesicle.

The most anterior of these is the rudiment of the fifth nerve (Figs. 2.5, V. 4.5, V). Its narrowed outer portion or

FIG. 45.

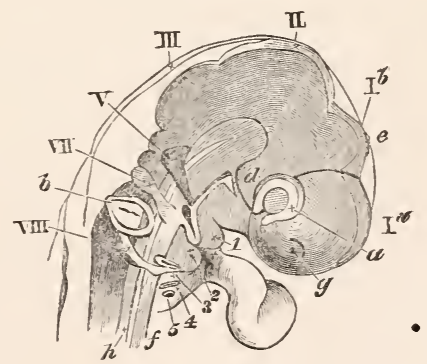

Head of an Eubrio Chick of the Third Day (seventy-five hours) rietred SIDEWAYS AS A TRANSPARENT OBJECT (from Huxley).

I $a$. cerebral hemispheres. I $b$, vesicle of the third ventricle. II. mid-brain. III. hind-brain. g. nasal pit. $a$. optic vesicle. $b$. otic vesicle. $d$. infundibulum. $e$. pineal body. $h$. notochord. V. fifth nerve. VII. seventh nerve. VIII. united glossopharyngeal and pneumogastric nerves. 1, 2, 3, 4, 5 the five visceral folds.

The stage here represented is a little later than that shewn in Fig. 25 , with which it should be compared. 
stalk divides into two bands or nerves. Of these one passing towards the eye terminates at present in the immediate neighbourhood of that organ. Compare Fig. 46. The other branch (the rudiment of the inferior maxillary branch of the fifth nerve) is distributed to the first visceral arch (Fig. 46). The second mass (Fig. 25, VII. 45, VII.) is the rudiment of the seventh, or facial nerve. It is the nerve of the second visceral arch.

The two masses behind the auditory vesicle represent the glossopharyngeal and pneumogastric nerves (Fig. 45, VIII., Fig. 46, $G$. $P h$. and $P g$.). At first united, they subsequently become separate. The glossopharyngeal supplies the third arch, and the pneumogastric the fourth arch.

These four masses, representing four important mixed cranial nerves, seem to be derived directly from the mesoblast surrounding the hind-brain. It is worthy of notice that they are mixed, sensory and motor, nerves; for, restricted as are the sensory functions of the seventh, and the motor functions of the pneumogastric in the adult mammal, the study of their comparative physiology leaves no doubt as to the essentially mixed nature of each. It is also worthy of note that of the third, fourth and sixth nerves, no such early rudinents appear; and there are reasons for thinking that these are in reality intercranial branches, the third and fourth of the fifth, and the sixth of the seventh nerves. The purely sensory nerve or rather sense-nerve, the auditory, seems to have a different origin altogether from all the above, though it may perhaps be looked upon as the dorsal branch of the seventh, while the erratic hypoglossal appears to be distinctly a spinal nerve.

Of the interesting relations of these cranial nerves to the visceral arches, we shall have to speak more fully in the second part of this work, when we describe the more primitive forms in the lower vertebrata.

At the same time that these ganglia make their appearance, or a little earlier, near the beginning of the third or end of the second day, there may be seen in the region of the hind-brain, lines which appear to divide off the mesoblast on either side into masses somewhat resemoling protovertebræ. Of these masses there are four or five on each side, generally three in front of. and two behind the optic vesicle. 'They were first noticed by Remak, and are easily distinguished from the rudimerts of the cranial nerves. 'They at first sight suggest the idea of an initial and transitory segmentation of the cranial mesoblast into protovertebre. It seems possible that they are, in reality, appearances produced by a series of very characteristic transverse wrinkles into which the walls of the hind-brain are at this time thrown, and which subsequently disappearing altogether, as the walls increase in thickness, may perhaps be viewed as indications of an aborted segmentation of the hind-brain into a series of vesicles. The true nature of these quadrate masses is still very problematical.

26. On the second day the newly formed Wolffian duct extended along the greater part of the length of the embryo as a tube resting on the mass of celis, which we have already called the intermediate cell-mass. 
On the third day, in consequence of the continually folding in of the somatopleure and especially of the splanchnopleure, as well as owing to the changes taking place in the protovertebræ, the Wolffian duct undergoes a remarkable change of position. Instead of lying as on the second day immediately under the epiblast (Fig. 20, W.d.), it is soon found to have apparently descended into the middle of the intermediate cell-mass (Fig. 41,w.d.) and at the end of the third day occupies a still lower position and even projects somewhat into the pleuroperitoneal cavity. (Fig. 4t, W.d.)

Towards the end of the day the rudiments of the Wolffian bodies (Fig. 44, W.b.) begin to make their appearance in connection with the ducts, but the consideration of these may conveniently be reserved to the next chapter.

27. The chief events then which take place on the third day are as follows:

1. 'The turning over of the embryo so that it now lies on its left side.

2. The cranial flexure round the anterior extremity of the notochord.

3. The completion of the circulation of the yolk-sac; the increased curvature of the heart, and the demarcation of its several parts; the appearance of new aortic arches, and of the cardinal veins. arches.

4. The formation of four visceral clefts and five visceral

5. The involution to form the lens, and the formation of the secondary optic vesicle.

6. The closing in of the otic vesicle.

7. The formation of the nasal pits.

8. The appearance of the vesicles of the cerebral hemispheres; the separation of hind-brain into cerebellum and medulla oblongata.

9. The completion of the fore-gut and of the hind-gut; the division of the former into œesophagus, stomach and duodenum, of the latter into large intestine and cloaca.

10. The formation of the lungs as two diverticula from the alimentary canal immediately in front of the stomach.

11. The formation of the liver and pancreas: the former as two diverticula from the duodenum, which subsequently 
become united by solid outgrowths; the latter as a single diverticulum also from the duodenum.

12. The changes in the protovertebræ and the appearance of the muscle-plates.

13. The appearance of the cranial nerves in the mesoblast adjoining the hind brain.

14. The change in position of the Wolffian duct. 


\section{CHAPTER VI.}

THE CHANGES WHICH TAKE PLACE DURING THE FOURTH DAY.

1. Ox opening an egg in the middle or towards the end of the fourth day, a number of points in which progress has been made since the third day are at once apparent. In the first place, the general growth of the embryo has been very rapid, so that its size is very much greater than on the previous day. In the second place, the white of the egg has still further diminished, the embryo lying almost in immediate contact with the shell membrane.

The germinal membrane embraces more than half the yolk, and the vascular area is about as large as a halfpenny.

Corresponding to the increased size of the embryo, there is a great increase in the quantity of blood circulating in the vascular area as a whole, though the sinus terminalis is already less distinct than it was.

2. The amnion becomes increasingly conspicuous. It is now seen as a distinct covering obscuring to a certain extent the view of the body of the chick beneath, and all traces of the junction of 'its folds are by this time lost. As yet there is very little fluid in the amniotic sac proper, so that the true amnion lies close upon the embryo.

3. The folding off of the embryo from the yolk sac has made great progress. The splanchnic stalk, which on the third day was still tolerably wide, inasmuch as about one third of the total length of the alimentary canal was as yet quite open to the yolk sac below, now becomes so much constricted by the progressive closing in of the splanchnopleure 
FIG. ${ }_{46 .}$

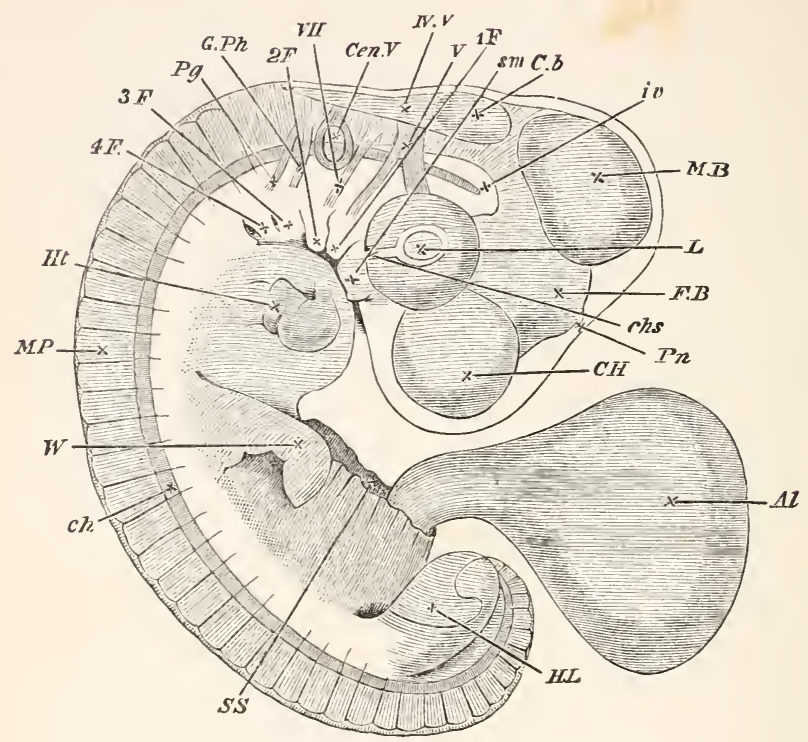

Eybryo at the End of the Fourth Day seen as a transparenm object.

The amnion has been completely removed, the cut end of the somatic stalk is shewn at S.S. with the allantois $(A l$.) protruding from it.

C.II. cerebral hemisphere. F. B. fore brain or vesicle of the third ventricle with the pineal gland $(P n$.$) projecting from its summit. M.B. mid brain.$ $C b$. cerebellum. $I V . V$. fourth ventricle. $L$. lens. ch.s. choroid slit. Owing to the growth of the optic cup the two layers of which it is composed cannot any longer be seen from the surface, but the posterior surface of the choroid layer alone is visible. Cen. V. auditory vesicle. s.m. superior maxillary process. I $F .2 F$. etc. first, second, third and fourth visceral folds. $V$. fifth nerve sending one branch to the eye, the ophthalmic branch, and another to the first visceral arch. VII. seventh nerve passing to the second visceral arch. $G$. $P h$. glossopliaryngeal nerve lassing towards the third visceral arch. $P$ g . pneumogastric nerve passing towards the fourth visceral arch. $i v$. investing mass. No attempt has leen made in the figure to indicate the position of the dorsal wall of the throat, which cannot be easily made out in the living embryo. $c h$. notochord. The front end of this cannot be seen in the living embryo. It does not end however as shewn in the figure, but takes a sudden bend d.wwnwards and then terminates in a point. Ht. heart seen through the walls of the chest. M.P. muscle-plates. W. wing. H.L. hind limb. Beneath the hind limb is seen the curved tail. 
folds, that the alimentary canal may be said to be connected with the yolk sac by a very narrow neck only. This remnant of the splanchnic stalk we may now call the umbilical duct; though narrow, it is as yet quite open, affording still free communication between the inside of the yolk sac and the interior of the alimentary canal.

The somatic stalk, though narrowing somewhat, is much wider than the splanchnic stalk, so that a considerable ring-shaped space exists between the two.

4. Another very prominent feature is the increase in the cranial flexure. During the third day, the axis of the front part of the head was about at right angles to the long axis of the body; the whole embryo being still somewhat retortshaped. On this day, however, the flexure has so much increased that the angle between the long axis of the body and that of the front segment of the head is an acute one, and the mouth is turned so as completely to face the chest.

The tail-fold, which commenced to be noticeable during the third day, has during this day increased very much, and the somewhat curved tail (Fig. 46) forms quite a conspicuous feature of the embryo. The general curvature of the body has also gone on increasing, and as the result of these various Hexires, the embryo has very much the appearance of being curled up on itself (Fig. 46).

5 . The distinct appearance of the limbs must be reckoned as one of the most important events of the fourth day.

Owing to the continued greater increase of depth than of breadth, the body of the embryo appears in section (Fig. 47) higher and relatively narrower than even on the third day, and the muscle-plates, instead of simply slanting downwards, come to be nearly vertical in position. Not far from the line which marks their lower ends, the somatopleure, almost immediately after it diverges from the splanchnopleure, is raised up (Fig. 47, W.R.) into a low rounded ridge which runs along nearly the whole length of the embryo from the neck to the tail.

It is on this ridge, which is known as the Wolffian ridge, that the limbs first appear as flattened conical buds projecting outwards. They seem to be local developments of the ridge, the rest of which becomes less and less prominent as they increase in size. Each bud, roughly triangular in sec- 
FIG. 47 .

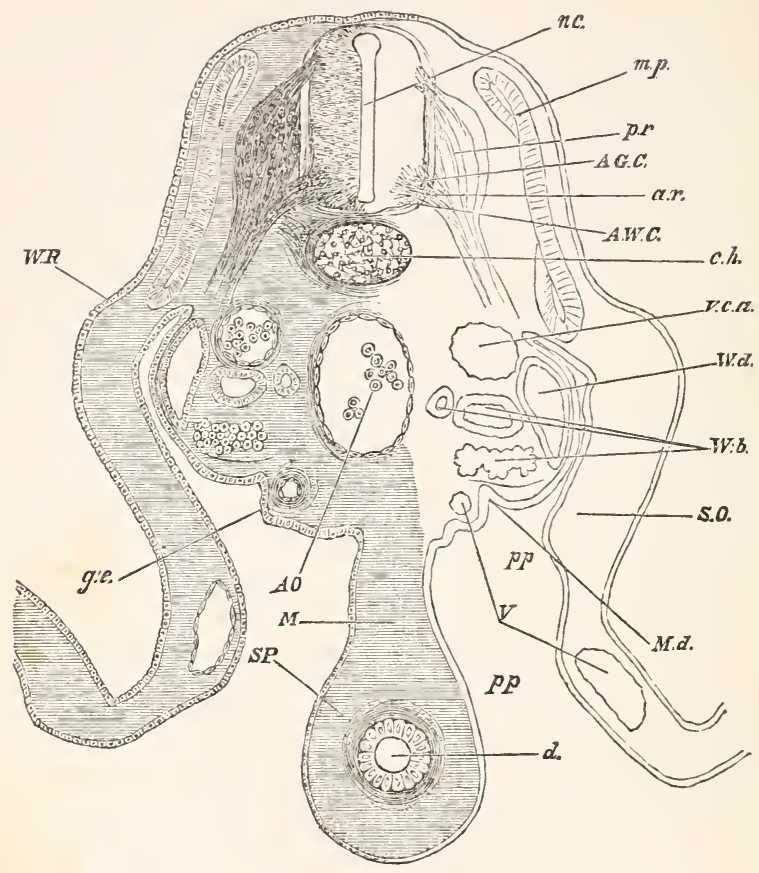

Section throdgh the Lumbar Region of an Embrto at the End of the Fourth Dax.

n.c. neural canal. $p . r$. posterior root of spinal nerve with ganglion. $a . r$. anterior root of spinal nerve. A. G.C. anterior grey column of spinal cord. A. W. C. anterior white column of spinal cord just commencing to be formed, and not very distinctly marked in the figure. m. p. muscle plate. ch. notochord. $W . R$. Wolffian ridge. A. O. dorsal aorta. $T^{\prime} . c . a$. posterior cardinal vein. $W . d$. Wolffian duct; its section is not circular, owing to its being cut through at a point where it is being joined by one of the tubules. W. $b$. Wolffian body, consisting of tubules and Malpighian corpuscles. One of the latter is represented on each side, that on the left hand having its glomerulus entirely filled with blood corpuscles. g. e. germinal epithelium. $M . d$. commencing involution of germinal epithelium to form the duct of Müller. $d$. alimentary canal. $M$. commencing mesentery. S. O. somatopleure. S.P. splanchnopleure. $V$. blood-vessels. $p p$. pleuroperitoneal cavity. 
tion, consists of somewhat dense mesoblast covered by epiblast which on the summit is thickened into a sort of cap. The front limbs or wings (Fig. 46) arise just behind the level of the heart, and the hind limbs in the immediate vicinity of the tail. The first traces of them can be seen towards the end of the third, but they do not become conspicuous till the fourth day, by the end of which the two pairs may be already distinguished by their different shapes. 'The front limbs are the narrowest and longest, the hind ones being comparatively short and broad. Both are flattened from above downwards and become more so as their growth continues.

6. In the head, the vesicles of the cerebral hemispheres are rapidly increasing in size, overlapping the insignificant olfactory vesicles in front, and encroaching on the 'tweenbrain or vesicle of the third ventricle behind. The mid-brain is now, relatively to the other parts of the brain, larger than at any other epoch, and an indistinct median furrow on its upper surface indicates its division into two lateral halves. The great increase of the mesoblastic contents of the secondary optic vesicle or involuted retinal cup causes the two eyeballs to project largely from the sides of the head (Fig. 48, Op). The mass of mesoblast which invests all the various parts of the brain, is not only growing rapidly below and at the sides, but is also undergoing developments which result in the formation of the primitive skull, and of which we shall speak in detail in a subsequent separate chapter. All these events, added to the cranial flexure spoken of above, give to the anterior extremity of the embryo a shape which it becomes more and more easy to recognize as that of a head.

7. Meanwhile the face is also being changed. The two nasal pits were on the third day shallow depressions with thickened borders complete all round. As the pits deepen on the fourth day by the growth upwards of their rims, a break is observed in each rim in the form of a groove ( $\mathrm{Fig} .48, N^{\top}$ ) directed obliquely downwards towards the cavity of the mouth. 'The fronto-nasal process or median ridge (Fig. 48 , $n f$ ), which on the third day rose up between the superficial projections caused by the bulging anterior extremities of the vesicles of the cerebral hemispheres, and on the fourth day becomes increasingly prominent, separates the two grooves from earh 
Fig. 48 .
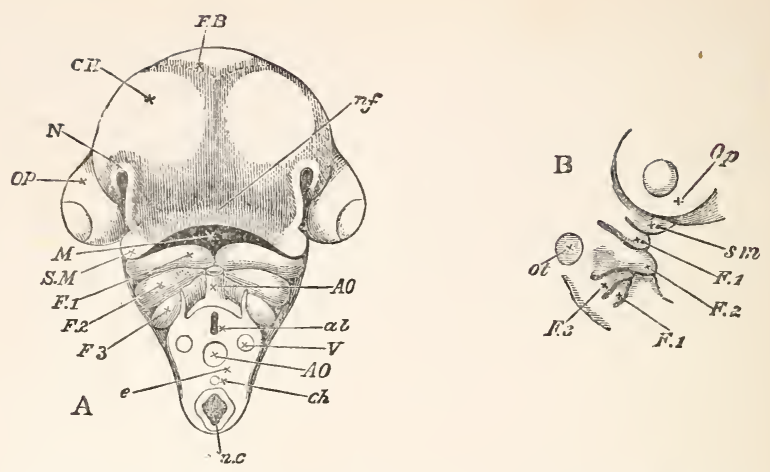

A. Head of an Embryo Chick of the Fourth Day viewed frour below AS AN OPAQUE OBJECT. (Chromic acid preparation).

$C H$. cerebral hemispheres. FB. vesicle of the third ventricle. Op. eyeball. $n f$. naso-frontal process. M. cavity of mnuth. S. M. superior maxillary process of $F$. I, the first visceral fold (inferior maxillary process). $F .2, F .3$. second and third visceral folds. $N$. nasal pit.

In order to gain the view here given the neck was cut across between the third and fourth visceral folds. In the section $e$ thus made, are seen the alimentary canal $a l$ with its collapsed walls, the neural canal n.c., the notochord $c h$., the dorsal aorta $A O$., and the vertebral veins $I$.

The incision has been carried just below the upper limit of the pleuroperitoneal cavity, consequently a portion of the somatopleure appears at the angle between the two third visceral folds. Almost embraced by the piece of somatopleure is seen the end of the bulbus arteriosus $A o$.

In the drawing the nasal groove has been rather exaggerated in its upper part. On the other liand the lower part of the groove where it runs between the superior maxillary process S.M. aud the broad naso-frontal process was, in this particular embryo. extremely shallow and indeed hardly visible. Hence the end of the superior maxillary process seems to join the inner and not, as described in the text, the outer margin of the nasal groove. A few hours later the separation of the two would have heen very visible.

I. 'The same seen silleways, to shew the visceral folds. Letters as before.

other, and helps to form the inner wall of each of them. Abutting on the outer side of each groove and so helping to form the outer wall of each, lie the ends of the superior maxillary processes of the first visceral arch (Fig. $48 \mathrm{~B}, \mathrm{sm}$ ), which like the fronto-nasal process are increasing in size. By their continued growth, the groove is more and more deepened, and leading as it does from the nasal pit to the cavity of the mouth, may already be recognized as the rudiment of the passage of the posterior nares. 
8. During the latter half of the fourth day there appears at the bottom of the deep lozenge-shaped cavity of the mouth, in the now thin wall dividing it from the alimentary canal, a longitudinal, or according to Götte a vertical slit which, soon becoming a wide opening, places the two cavities in complete communication.

The cavity of the mouth, being, it will be remembered, formed partly by depression, partly by the growth of the surrounding folds, is lined entirely with epiblast, from which the epithelium of its surface and of its various glands is derived. In this respect, as Remak pointed out, it differs from all the rest of the alimentary canal, whose whole epithelium is formed out of hypoblast.

9. By the side of the hind-brain, in which the cerebellum, through the increased thickening of its upper walls, is becoming more and more distinct from the medulla oblongata, both in front and behind the auditory vesicle, in which the rudiments of the cochlea and recessus vestibuli are already visible, the cranial ganglia and nerves are acquiring increased distinctness and size. They may be very plainly seen when the head of the fresh embryo is subjected to pressure.

The foremost is the fifth cranial nerve (Fig. 46, V.) with its Gasserian ganglion; it lies a little way behind the anterior extremity of the notochord immediately below the cerebellum. Next to this comes the seventh (Fig. 46, VII.) nerve, starting just in front of the ear-vesicle, and extending far downwards towards the second visceral arch. The two nerves which lie behind the ear-vesicle are now obviously separate from each other; the front one is the glossopharyngeal (Fig. 46, G. Ph.), and the hinder one already shews itself to be the pneumogastric (Fig. 46, Pg.).

10. Besides the progressive changes of the alimentary canal and its surroundings, which we incidentally described in the last chapter, and the closure of the mid-gut to form the umbilical duct, of which we have also already spoken, a totally new and most important appendage of the digestive tract, the allantois, becomes for the first time conspicuous on this day, though the first rudiments of it appeared on the third.

Soon after its appearance the allantois may easily be recognized as a pear-shaped vesicle lying in the hinder district of the pleuroperitoneal space, and connected with the under 
surface of the cloaca by a long hollow stalk, which places its cavity in communication with that of the alimentary canal. Both vesicle and stalk have an outer coat of mesoblast and an inner lining of what apparently is hypoblast. So much any observer may readily determine for himself; but of the earliest stages of the development of this organ different embryologists have given very different accounts.

Von Baer believed that, soon after the cloaca was formed by the enlargement of the cæcal hind end of the alimentary canal, the allantois arose from it as a spherical diverticulum, generally visible about the middle of the third day, in whose formation both of the coats of the alimentary canal took part. This spherical diverticulum gradually lengthened out into a pear-shaped vesicle, connected with the cloaca by a hollow stalk which rapidly narrowed and lengthened, until the allantois formed an independent hollow body, composed of an outer coat of mesoblast and a lining of hypoblast, and communicating with the cloaca by a narrow tube of the same construction.

Reichert (Entwicklungsgeschichte, s. I 86) on the other hand stated that the allantois was formed of two solid outgrowths from the mesoblast of the somatopleure, which subsequently coalesced and became hollow; but believed that it was primarily connected with the Wolffian ducts and not with the cloaca.

According to Remak (Entwicklung, $\$ 57,58$ ) it is formed by two solid vaccular outgrowths of the mesoblast of the body-wall, one on each side of the middle line, which project in the pleuroperitoneal cavity near to the cloaca. 'These two outgrowths coalesce, and then grow up, till they come in contact with

FIG. 49.

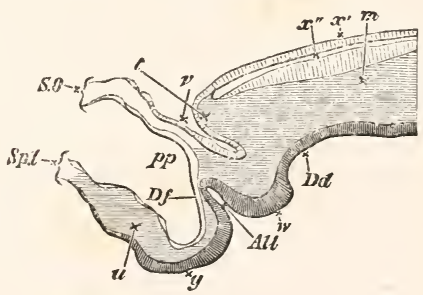

Longitudinal Section of the Tail-end of an Embryo Chick at the commencement of the Third Day (Dobryniu).

$t$. the tail, $m$. the axial mesoblast of the body, about to form the protovertebræ. $x^{\prime}$. the roof of $x^{\prime \prime}$. the neural canal. $D d$. the hind end of the lind-gut. SO. somatopleure. Spl. splanchnopleure. $u$. the mesoblast of the splanchnopleure carrying the vessels of the yolk-sac. $P p$. pleuroperitoneal cavity. If. the epithelium lining the pleuroperitoneal cavity. All. the commencing allantois. $w$. and $y$. the hypoblast thickened and projecting on either side of the opening of the allantois. 
the wall of the cloaca: with this they unite, and form together a soliel spherical body, bearing on its external surface a median furrow, indicating its double origin. A narrow diverticulum of hypoblast now passes into the mass, and forms within it a cavity, which is at first sinall and, corresponding to the ex. ternal contour of the body, to a certain extent double. The hypoblast diverticulum grows rapidly, while its mesoblastic covering remains nearly stationary, so that the mesoblast finally comes to form a thin coating only over the hypoblast.

His (op. cit.) gives a somewhat elaborate and complicated account of the development of the allantois; which is accepted by Waldeyer (Eierstock und Ei) and Bornhaupt (Untersuchung über die Entwickelung des Ürino-genitalsystems: beim Hüllncken, Riga, 1867).

It appears to be nearly the same as the fuller account given by Dobrynin (Ueber die erste Anlage der Allantois. Sitz. der $\mathrm{k}$. Akad. Wien, Bd. $6_{+}$, IS7I), of which the following is an abstract.

When the first commencement of the hind fold takes place, immediately beyond the point where the hypoblast turns back to assume its normal direction over the yolk-sac, a narrow diverticulum which points backwards and somewhat upwards is formed by a special tlexure of the splanchnopleure. The open end of the diverticulum, Fig. 49, All., looks forwards towards the wide opening connecting the digestive tract with the yolk-sac; its blind end points directly towards the pleuroperitoneal cavity. This diverticulum is the commencing allantois. It is lined by hypoblast, while its exterior is composed of the mesoblast of the splanchuopleure.

As the folding in to forn the digestive tract increases, the diverticulum alters

FIG, 50.

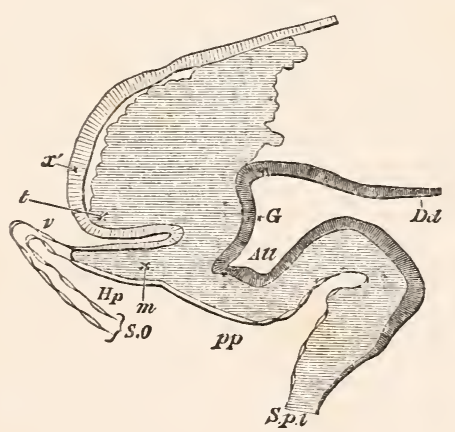

Longitudinal Section of the Tall-end of an Embrí Chick at the MIDDLE OF THE THIRD DAY (Dobrynin).

$t$. the tail; the line of reference points to the axial mesoblast at the tail. $x^{\prime}$. epiblast. SO. somatopleure. $m$. mesoblast to form the body wall. $V$. commencing amniotic fold. $H p$. space between the true and the false amnion. $p p$. Pleuroperitoneal cavity. Spl. splanchnopleure. G. Cloacal enlargement of the alimentary canal. $D d$. dorsal wall of the alimentary canal. All. vesicle of the allantois having a wide opening into the alimentary canal. 
its position and becomes quite parallel with the commencing digestive tract. Its cavity is separated from that of the digestive canal by a projection of mesoblast covered by hypoblast; but both open freely in front into the common splanchnic stalk.

In the next stage it still further alters its position, and forms, Fig. 5o, a rather wide vesicle lying immediately below the hind end of the digestive canal, with which it communicates freely by a still broad opening; its blind end projects freely into the pleuroperitoneal cavity below. It was in this condition when Von Baer first observed it.

At the time when these changes are taking place, the somatopleure is being folded in to form the walls of the body; and as the folds, one on either side, are carried forward from the extreme end of the tail, they present themselves, when seen from within or in sections, as two ridges projecting towards the sides of the allantois. Reaching the allantois these ridges fuse with its wall, and in this way reduce the pleuroperitoneal cavity immediately below the allantois to quite a narrow space, which is seen in section as a mere chink. Remak apparently mistook these infoldings of the somatopleure, and the consequent projections into the pleuroperitoneal cavity, for the first formation of the allantois, although they liave in fact little or no connection with it.

We may therefore probably consider the allantois as a portion of the cloaca, which grows forward and becomes an independent spherical vesicle, still however remaining connected with the cloaca by a narrow canal which forms its neck or stalk. The opening of the allantois into the cloaca is on the under side of the latter. Both the neck and resicle of the allantois are lined by liypoblast, while its exterior is composed of the mesoblast of the splanchnopleure. From the first the allantois lies in the pleuroperitoneal cavity. In this cavity it grows forwards till it reaches the front limit of the hind-gut, where the splanchnopleure turns back to reach the yolk-sac. It does not during the third day project beyond this point; but on the fourth day begins to pass out beyond the body of the chick along the as yet wide space between the splanchnic and somatic stalks of the embryo on its way to that space between the external and internal folds of the amnion, which it will be remembered is directly continuous with the pleuroperitoneal cavity (Fig S, $K$ ). In this space it eventually spreads out over the whole body of the chick. On the first half of the day the vesicle is still very small, and its growth is not very rapid. Its mesoblast wall still remains very thick. In the later half of the day its growth becomes very rapid and it forms a very conspicuous object in a chick of that date (Fig. 46, $A l$.). At the same time its blood-vessels become important. To these we shall presently return. 
11. The protovertebræ, which by the continued differentiation of the axial mesoblast at the tail end of the embryo have increased in number from thirty to forty, undergo during this day changes of great importance. Since these changes are intimately connected with the subsequent development of the vertebral column, it will perhaps be more convenient to describe briefly here the whole series of events through which the protovertebræ become converted into the permanent structures to which they give rise, though many of the changes do not take place till a much later date than the fourth day.

The separation of the muscle-plates (Chap. v. § 23) left the remainder of each protovertebra as a somewhat triangular mass lying between the neural canal and notochord on the inside, and the muscle-plate and intermediate cell-mass on the outside (Fig. 44). Already on the third day the upper angle of this triangle grows upwards, between its muscleplate and the neural canal, and meeting its fellow in the middle line above, forms a roof of mesoblast over the neural canal, between it and the superficial epiblast. At about the same time, the inner and lower angle of the triangle growis inwards towards the notochord, and passing both below it (between it and the aorta) and above it (between it and the neural canal), meets a similar growth from its fellow protovertebra of the other side, and thus completely invests the notochord with a coat of mesoblast, which, as seen in Fig. 47 , is at first much thicker on the under than on the upper side.

While the inner portion of each protovertebra is thus extending inwards around both notochord and neural canal, the remaining outer portion is undergoing a remarkable change. It becomes divided into an anterior or præaxial, and a posterior or postaxial segment. The anterior, which is the larger and more transparent of the two, is the rudiment of the spinal ganglion and nerve, while the posterior, which remains more particularly connected with the extensions round the neural canal and notochord, goes to form part of the permanent vertebra.

In this way, each protovertebra, having given rise to a muscle-plate, is further divided into a ganglionic rudiment, and into a mass which we may speak of as a "primary" 
vertebra, consisting as it does of a body or mass investing the notochord, from which springs an arch covering in the neural canal.

Both body and arch consist at this epoch of but slightly clifferentiated mesoblast, and the arch springs, to a certain extent, not only from the posterior segment of the protovertebra, but also from the anterior or ganglionic segment: though, as seen in Fig. 47, it is far less conspicuous at the level of the latter than of the former. Both neural canal and notochord are thus furnished from neck to tail with a complete investment of mesoblast, still marked, however, by the transparent lines indicating the fore and aft limits of the several protovertebræ. This is sometimes spoken of as the "membranous" vertebral column.

The ganglionic rudiment, placed anteriorly to its corresponding primary vertebra, consists in chief of a large oval swelling, the ganglion of the posterior root (Fig. 47, pr). At a little distance beyond its ganglion, the posterior root is joined by the anterior root (ar); and the two form together the common nerve-trunk, which is at first very short. Compared with either root or with the nerve-trunk the ganglion, at this epoch and for some time afterwards, seems disproportionably large. At first, neither root is connected with the involuted epiblast of the neural canal. Very speedily, however, they both come to be united with that portion of the neural tube which, as we shall presently state, gives origin to the grey matter of the spinal cord. It is, however, easier to trace the fibres of the anterior root into the cord, than those of the posterior, and they can be followed in it for a greater distance.

On the fourth day the nerves are composed of cells whose protoplasm is beginning to become converted into fibres. Amongst these fibres, the nuclei of the cells with distinct nucleoli are thickly seattered. On the sixth day and still more on the seventh the fibrillated structure of the nerves is much more distinct and the nuclei are far less numerous.

The ganglia on the fourth day are composed of numerous nuclei surrounded by protoplasm, between which the fibres of the nerves pass. Covering this mass of nucleated cells is a layer of mesoblast (also derived from the tissue of the protovertebra) which, by the sixth day, forms a kind of sheath around them.

The cells of the ganglia from the fourth to the sixth day contain round granular nuclei with distinct nucleoli very similar to the nuclei of the ordunary mesoblast-cells. The limits of the protoplasm of the individual cells are as a general rule not easily seen, but with care may be made out. The amount of protoplasm round each nucleus appears however to be very small. 
The fihres of the nerve can eacily be traced through the ganglion. In section they appear to have a sımewhai wavy course, and by interlacing divide the ganglinn into a number of elongated areas in each of which is a row of nuclei. In sections of the sixth day it is not possible to trace a connection between the nerve-fibres and the cells. The nuclei are must numerous at the lower ends of the ganglia.

On the seventh day, the nuclei have become larger, and where the outline of a cell can be distinctly seen it is generally somewhat angular. In sections it is still on the seventh day not possible to trace any conuection between the cells and the nerve fibres.

Remak (op. cit.) speaks of the ganglia heing composed of non-nucleated spheres, and Lockhart Clarke (Philosoplical Transactions, 1862) also describes the ganglia-cells as "celis or nuclei" which are at tirst mere rounded masses of protoplasm, and do not arquire a nucleus till a later period. Both of theso statements are according to our observations incorrect.

In the later stages according to Lockhart Clarke's account (loc. cit.) the cells of the ganglia send out processes which anastomose together into a fine network. The ceils also become connected with the nerve fibres, which can sometimes be seen to divide in the ganglion into a fine brush-like bundle of fibrillæ. At this time the cells possess a distinct nucleus and nucleolus. These changes he describes as completed by the ninth day of incubation.

His believes that the spinal nerves are derived from dowuward prolongations of the superficial epiblast descending between the protovertebræ. This view has not been corroborated by subsequent observers.

\section{The remaining portions of the protovertebre form-} ing the primary vertebræ or membranous vertebral column spoken of in the last paragraph, are converted into the permanent vertebræ; but their conversion is complicated by a remarkable new or secondary segmentation of the whole vertebral column.

On the fourth day, the transparent lines marking the fore and aft limits of the protovertebræ are still distinctly visible. On the fifth day, however, they disappear, so that the whole vertebral column becomes fused into a homogeneous mass whose division into vertebræ is only indicated by the series of ganglia. This fusion, which does not extend to the muscle-plates in which the primary lines of division still remain visible, is quickly followed by a fresh segmentation, the resulting segments being the rudiments of the permanent vertebræ. The new segmentation, however, does not follow the lines of the earlier division, but passes between the ganglionic and the vertebral portions, in fact, through the middle, of each protovertebra. In consequence, each spinal ganglion and nerve ceases to form the front portion of the primary vertebra, formed out of same protovertebra as itself, but is attached to the hind part of the permanent vertebra 
immediately preceding. Similarly, the rudiment of each vertebral arch covering in the neural tube no longer springs from the hind part of the protovertebra from which it is an outgrowth, but forms the front part of the permanent vertebra, to which it henceforward belongs. The ganglia are still, however, the most conspicuous portions of each segment.

By these changes this remarkable result is brought about, that each permanent vertebra is formed out of portions of two consecutive protovertebræ. Thus, for instance, the tenth permanent vertebra is formed out of the hind portion of the tenth protovertebra, and the front portion of the eleventh protovertebra, while its arch, now attached to its front part, was attached to the hind part of the tenth protovertebra.

The new segmentation is associated with or rather is caused by histological changes. At the time when the fusion takes place, the mesoblast, which in the form of processes from the protovertebral bodies surrounds and invests the notochord, has not only increased in mass but also has become cartilaginous, so that, as Gegenbaur (Untersuchung zur vergleichenden Anatomie der Wirbelsüule bei Amphibien und Reptilien, Leipzig, 1862) points out, we have for a short period on the fifth day a continuous and unsegmented cartilaginous investment of the notochord.

This cartilaginous tube does not however long remain uniform. At a series of points corresponding in number to the original protovertebre it becomes connected with a number of cartilaginous arches which appear in the protovertebral investment of the neural canal. These arches, which thus roof in the neural canal and each of which arises opposite to the vertebral portion of each protovertebral body, are the cartilaginous precursors of the osseous vertebral arches. We further find that the portions of the cartilaginous tube from which the arches spring come to differ histologically from the portions between them not connected with arches: they are clearer and their cells are less closely packed. There is however at this period no distinct segmentation of the cartilaginous tube, but merely a want of uniformity in its composition.

The clearer portions, from which the arches spring, form the bodies of the vertebre, the segments between them the intervertebral regions of the column. 


\section{VI.] SECONDARY SEGMENTATION OF VERTEBRAL COLUMN. 15.j}

W. Schwarck (Beiträge zur Entwicklung der Wirbelsäule bei den Tögeln. Anatomische situdien, Dr Hasse, III. Heft, IS72) states that both in the intervertebral and the vertebral segments the cartilage is diviled into tuo layers, an imner, central, and an outer peripheral. This division is less marked in the intervertebral than in the vertebral region.

The inner layer in the vertebral region he speaks of as "the body of the vertebra belonging to the notochord," and the external layer as "the skeletonforming layer."

On the fifth day a division takes place of each of the $i n-$ tervertebral segments into two unequal parts; a larger one appertaining to the vertebra in front and a smaller one to the vertebra behind. To the larger segment the spinal ganglia naturally remain attached, and thus comes about the alteration of their place in relation to the vertebræ which we before spoke of.

This fresh segmentation is not well marked, if indeed it takes place at all in the sacral region.

Each arch at its first appearance corresponds to about the middle of a vertebral portion of a protovertebra, but after the secondary segmentation the portion of each vertebra behind its arch grows more quickly than that in front, and thus after a while the arches seem to spring from the front rather than from the middle of the vertebral segments.

To recapitulate:- the original protovertebræ lying side by side along the notochord, after giving off the muscle-plates, and dividing lengthways into ganglionic and vertebral portions, grow around, and by fusing together completely invest, with mesoblast of protovertebral origin, both neural canal and notochord.

This investment, of which by reason of its greater growth the original bodies of the protovertebræ seem to be only an outlying part, becomes cartilaginous in such a way that while the notochord becomes surrounded with a thick tube of cartilage bearing no signs of segmentation, but having the ganglia lodged on its exterior at intervals, the neural canal is covered in with a series of cartilaginous arches, connected to each other by ordinary mesoblastic tissue only, but passing at their bases directly into the cartilaginous tube around the notochord.

By a histological process of differentiation the cartilaginous tube is divided into vertebral and intervertebral portions, the vertebral portions corresponding to the arches 
over the neural canal. Fresh lines of segmentation then appear in the intervertebral portions, which run in such a way that each ganglion is now more closely associated with the vertebral portion in front of it than with that belind it, though the latter sprang in part from the same original protovertebra as itself.

1:3. Meanwhile from the fourth to the sixth day important changes take place in the notochord itself.

On its first appearance the notochord was, as we have seen, composed of somewhat radiately arrariged but otherwise perfectly typical mesoblast-cells.

On the third day some of the central cells become vacuolated, while the peripheral cells are still normal. The vacuolated cells exhibit around the vacuole a peripheral layer of granular protoplasm in which the nucleus lies embedded, whilst the vacuoles themselves are filled with a perfectly clear and transparent material, which in an unaltered condition is probably fluid. To riards the end of the day the notochord acquires a delicate structureless sheath which is no doubt a product of its peripheral cells.

According to $\mathrm{His}$ there is a cavity in the centre of the notochord on the third day. We have never observed this, and it is denied by Miiller (Úcber den Bau der Chorda Jorsalis. Jenaische Zeitschrift. Bd. vI. 1871).

On the fourth day all the cells become vacuolated with the exception of a single layer of flattened cells at the periphery; and the vacuoles themselves become larger. At the point where the nucleus lies there is generally rather more protoplasin than round the remainder of the circumference of the cells.

On the sixth day all the cells are vacuolated. In each cell the vacuoles have so much increased at the expense of the protoplasm that only a very thin layer of the latter is left at the circumference of the cell, at one part of which, where there is generally more protoplasm than elsewhere, the starved remains of a nucleus may generally be detected.

Miiller (loc. cit.) considers that the cells have a membrane. This however is probably merely a hardened external layer of the protoplasm; and is stained by reagents.

Dursy (Zur Entwicllungsgesclichte des Kopfes des Menschen und der höheren Wirbelthiere) believes that what we have spoken of as vacuoles in the cells are really intercellular spaces. So that according to his view the notochord is composed of stellate cells with large round intercellular spaces filled with transparent intercellular matter. Superficially viewed a section of the noto- 
chord of the sixth day might be supposed to have such a structure, but the study of its development and a careful examination of its structure proves that this is not a correct account.

Accorling to the measurements of Müller (loc. cit.) the diameter of the notochord on the third day is $0.09 \mathrm{~mm}$. and that of the central cells $0.012-$ 0.018 . On the fourth day the notnchord is $0.16 \mathrm{~mm}$. in diameter and its component cells are also larger. Un the sixth day its diameter is at the maximum and reaches $0.2 \mathrm{~mm}$. The central cells measure $0.02 \mathrm{~mm}$.

From these measurements it will be seen that the vacuolation of the cells of the notochord is accompanied by a rapid growth both in the size of the cells and in the diameter of the notochord itself.

14. The notochord is on the sixth day at the maximum of its development, the change which it henceforward undergoes being of a retrograde character.

From the seventh day onward, it is at various points encroached upon by its investment. Constrictions are thus produced which first make their appearance in the intervertetral portions of the sacral region. In the cervical region, according to Gegenbaur, the intervertebral portions are not constricted till the ninth day, though as early as the seventh day constrictions are visible in the vertebral portions of the lower cervical vertebra. By the ninth and tenth days, however, all the intervertebral portions have become distinctly constricted, and at the same time in each vertebral portion there have also appeared two constrictions giving rise to a central and to two terminal enlargements. In the space therefore corresponding to each vertebra and its appropriate intervertebral portion, there are in all four constrictions and three enlargements.

On the twelfth day the ossification of the bodies commences. At that time, according to Schwarck (loc. cit.), the cartilaginous bodies of the vertebræ are composed of an inner layer in which the cells form lines radiating from the notochord, and an outer layer somewhat sharply separated from the inner one. In the inner layer, immediately around the notochord, ossification first commences.

Gegenbaur (loc. cit. p. $6_{\boldsymbol{\tau}}$ ) considers that this lajer in which ossification commences corresponds to the primordial body of the vertebre in amphibians. Schwarck is doubtful whether it corresponds to his imner liyer of cartiliage in the first stage.

In rare cases ossification first commences as a deposit on the exterior of the vertebræ.

The first vertebra to ossify is the second or third cervical, and the ossification gradually extends backwards. It does 
not commence in the arches till somewhat later than in the bodies. For each arch there are two centres of ossification, one on each side.

We may remind the reader that in the adult bird we find between each of the vertebræ of a neck and back a cartilaginous disc-the meniscus-which is piercert in the centre. These discs are thick at the circumference but thin oif to a fine edye round the central hole. Owing to the shape of these discs there are left between each pair of vertebræ $t$ wo cavities, which only communicate through the central aperture of the meniscus. Through this central aperture there passes a band connecting the two vertebræ which is called the 'ligamentum suspensorium.'

In the tail the inenisci are replaced by borlies known as the 'annuli fibrosi,' which precisely resemble the sinilarly named bodies in mammals. They differ from the menisci in being attached over their whole surface to the ends of the vertebral bodies, so that the cavities between the menisci and the vertebræ cease, to exist. They are pierced however by a body corresponding with the ligamentum suspensorium and known as the 'nuclens pulposus.'

In the inte vertelural regions the chorda, soon after the commencement of ossification, $t$ tirely disappears. The cartilage around it however becomes converted (in the region of the neck) into the ligamentum suspensorium, which unites the two vertebræ between which it is placed.

In the tail the cartilage becomes the nucleus pulposus, which corresponds exactly to the 'ligamentum suspensorium' of the neck and back.

Shortly after the formation of the ligamentum suspensorium the remaining cartilage of the intervertebral segments is converted in the neck and back into the meniscus between each two vertebræ, and in the tail into the annulus fibrosus. Both are absent in the sacrum. These points together with the anatomy of these parts in the adnlt were first made out by Jäger (Wirbellörpergelenk der Vöyel. Sitz. der k. Akad. Wien, vol. xxxiı. I859).

In the bodies of the vertebre the notochord does not entirely disappear as in the intervertebral regions, but, according to Gegenbaur, undergoes ultimately a direct conversion into cartilage. The contour of the sheath becomes indistinct; the cells by the accumulation of matrix round them take on the f egg the limits between the altered notochord and the cartilage of protovertebral origin can only with difficulty be made out.

15. While the chief mass of a protovertebra, having given rise to a muscle-plate and a ganglion, is converted into the body and arch of a permanent vertebra with its several appurtenances, a small portion of the exterior grows downwards as the rudiment for the formation of a rib. These costal growths are of course confined to the dorsal region. They are seen on the sixth day as cartilaginous rods, whose cells are arranged in horizontal rows. By this time they are quite separate from the bodies of the vertebra, with whose arches they are in transverse section seen to alternate. Thus in one section the vertebral arch will be distinctly seen but no trace of the rib; while in the next the rib will be visible but the arch will be absent. 
16. We shall conclude our account of the protovertebræ by describing the changes which take place in the muscleplates.

In the chick these are somewhat complicated, and have not been fully worked out.

On the third day the muscle-plates end opposite the point where the mesoblast becomes split into somatopleure and splanchnopleure. On the fourth day however (Fig. $\$ 7 \mathrm{mp}$.) they extend to a certain distance into the side walls of the body beyond the point of the division into somatopleure and splanchnopleure.

Into what muscles of the trunk they become converted has been somewhat disputed. There is no doubt that it is only episkeletal muscles, to use Professor Huxley's term (Vertebrates, p. 46), that are derived from them, but some embryologists have stated that they only form the muscles of the back. We have, however, little doubt that all the episkeletal muscles are their products; a view also adopted by Professors Huxley and Kölliker.

According to Kölliker the muscle-plates give rise to ( $\mathrm{I}$ ) the deep dorsal muscles, such as the semispinalis multifidus \&c., and (2) the visceral muscles as represented by abdominal muscles, the muscles of the breast, the superficial muscles of the neck, and the muscles of the jaws and face.

The front dorso-lateral (hyposkeletal) muscles, according to Kölliker, are derived from a front (ventral) muscle-plate, which is formed from the most ventral portion of the protovertebræ, but is very limited in extent in the fowl. These muscles include the longus colli, the recti antici, and quadratus.

This view differs from that of Huxley, chiefly in considering only the ventral dorsal muscles as hyposkeletal, and uot also the inner visceral muscles. Huxley believes that all the episkeletal muscles are derived from the muscleplates, but does not give an opinion as to the cells of the embryo from which the hyposkeletal muscles take their origin.

His takes an entirely different view; he believes that the muscles of the back only are derived from the muscle-plates, but that the muscles of the sides and ventral walls of the body are formed from the mesoblast of the somatopleure.

There can be little doubt that the intrinsic muscles of the limbs are not outgrowths from the muscle-plates, but are formed independently in the mesoblastic tissues of which the limbs are composed.

The origin of the extriusic limb-muscles is not so certainly known.

The cutaneous muscles are obviously derived from the original mesoblast of the somatopleure.

It seems very probable (though the subject has not yet been worked out) that the hyposkeletal voluntary muscles underlying the vertebral column are derived from the intermediate cell-mass, which originally lies externally to the protovertebræ, but into which, as we have before said, the cleavage of the mesoblast does not extend.

In the first instance, as is clear from their mode of origin, the muscle-plates correspond in number with the protovertebræ, and this condition is permanent 
in the lower vertebrates, such as fishes, where we find that the lateral muscle is divided by septa into a series of segments corresponding in number with the vertebre.

17. Of all the events of the fourth day, none perhaps are more important than those by which the rudiments of the complex urinary and generative systems are added to the simple Wolffian duct and b dy, which up to that time are the sole representatives of both systems.

We saw that the duct arose on the second day as a solid ridge which subsequently became a tube, lying immediately underneath the epiblast above the intermediate cell-mass, close against the upper and outer angles of the protovertebræ, and reaching from about opposite to the fifth protovertebra away to the hinder end of the embryo.

The exact manner in which it first appears is as yet a matter of dispute, and in our account of the second day, we gave the views of the majority of embryologists who have written on the subject. But it may be considered as quite certain that the Wolffian duct is formed out of mesoblastcells. It is most probable that the ridge is primarily formed by simple aggregation of cells, and that it is converted into a tube by its central cells taking on a radiating arrangement round a central hole, which is at first small but rapidly increases in size. In whatever way it be really formed, we find before the end of the second day, in the place of the previous ridge, a duct with a distinct though small limen. Waldeyer and some other observers have incorrectly stated that the lumen is not formed till somewhat later.

At first the duct occupies a position immediately underneath the superficial epiblast, but very soon after its formation the growth of the protovertebre and the changes which take place in the intermediate cell-mass, together with the general folding in of the body, cause it to appear to change its place and travel downwards (Chap. v. $\S 26$ ). While this shifting is going on, the cells lining the upper end of the pleuroperitoneal cavity (the kind of bay which, as seen in sections, is formed by the divergence of the somatopleure and splanchnopleure) become columnar, and constitute a distinct cpithelium. This epithelium, which is clearly shewn in Fig. 41, g.e, and is also indicated in Fig. 44, is often called the germinal epithelium, because some of its cells subsequently take 
part in the formation of the ovary. Soon after its appearance, the intermediate cell-mass increases in size and begins to grow outwards into the pleuroperitoneal cavity, as a rounded projection which lies with its upper surface towards the somatopleure, and its lower surface towards the splanchnopleure, but is in either case separated from these layers by a narrow chink. The Wolifian duct (Fig. 44, Wd, 47, $\left.W^{\prime} d\right)$ travels down, and finally before the end of the third day is found in the upper part of this projection, near that face of it which is turned towards the somatopleure.

At, or before, the fourth day, when the duct occupies this new position, the Wolffian body begins to be formed in the midst of the intermediate cell-mass.

The structure of the fully developed Wolffian body is fundamentally similar to that of the permanent kidneys, and consists essentially of convoluted tubules, commencing in Malpighian bodies with vascular glomeruli, and opening into the duct. It is formed as follows.

From the anterior portion of each duct and on its inner side, diverticula are given out at right angles. These gradually lengthen, and becoming twisted form the tubules, while the glomeruli of the Malpighian corpuscles seem to be derived from cells of the intermediate mass, which also gives rise to the vascular networks round the tubules.

The tubules, which from their contorted course are in sections (Figs. 47, 51) seen cut at various angles, possess an epithelium which is thicker than that of the Wolffian duct. From this difference it is generally easy to distinguish the sections of the tubules from those of the duct. The glomeruli of the Malpighian bodies are in sections of hardened embryos usually filled with blood-corpuscles.

In the above statements we have followed Waldeyer (Eierstock und Ei), but it ought to be mentioned that the majority of earlier observers have believed that the tubules arise independently in the mesoblast, and only at a later periorl become connected with the duct. The sections which Waldeyer has drawn seem however strongly to support the view which he has brought forward; our own sections also confirm it, and we have noticed that even before the formation of the tubules, the Wolffian duct exhibits great variations in diameter, being in some cases crescent-shaped in section, in others round; this seems clearly to indicate the giving off of diverticula. Waldeyer's observations have moreover been since confirmed by other observers.

The Wolffian body, as distinct from the duct, reaches from about the level of the fifth protovertebra to beyond the 
hind limbs; but the duct itself is carried on still further back.

Towards the hind end of the embryo, the projection of the intermediate cell-mass spoken of above becomes smaller and smaller, and the Wolffian duct is thus brought nearer to the splanchnopleure, and in the region of the hind-gut comes to lie close to the walls of the alimentary canal. On the fourth day, the two ducts meet and open into two horns, into which the side-walls of the recently formed cloaca are at that time produced, one on either side.

As we shall afterwards see, the duct of the permanent kidneys and Müller's duct also fall into these two horns of the cloaca.

The Wolffian bodies thus constituted perform the offices of kidneys for the greater part of embryonic life. In the chick they disappear before birth; but in most of the Ichthyopsida they remain for life as the permanent kidneys.

18. Near the end of the fourth day, on the outer surface of the projection formed by the Wolffian body a furrow is formed immediately below the Wolffian duct by an involution of the germinal epithelium. This furrow, which is shewn at $M . d$ in Fig. 47, deepens, and its walls arch over and unite. In this way a tube is formed, which separates from the germinal epithelium in the same way that the neural tube separated from the external epiblast. It is known as the Duct of Ilüller; of its function we shall speak later on.

This account of the origin of Müller's duct is due to Waldeyer (loc. cit.), whose observations have been confirmed by subsequent inquirers. An examination of our own sections leads us to the same conclusions.

Dr Sernoff (Centralblatt für Med. Wiss. $27 \mathrm{Jun} . \mathbf{1}_{74}$ ) agrees with Bornhaupt (Untersuchung über die Entwiclielung des Uino-genitalsystems beim Hülnchen) in considering that the duct of Müller is formed by a simple involution from the pleuroperitoneal cavity which grows backwards in the mesoblast between the Wolffian duct and the germinal epithelium; and thinks that Waldeyer is in error in supposing the involution to be in the form of an elongated furrow. This divergence of opinion is not of great importance compared with the point on which both observers are in agreement, viz. that the duct of Muiller is formed by an involution of the germinal epithelium from the pleuroperitoneal cavity.

The formation of the duct of Miiller takes place from before, backwards; but near the hind end of the embryo, where the germinal epithelium is deficient, the groove to form the duct becomes an involution which, at first solid 
but subsequently hollow, bores its way through the mesoblast, and finally appears to unite on the seventh day with the Wolffian duct close to the entrance of the latter into the cloaca. Later on, this state of things becomes altered; the duct of Muiller opens directly into the cloaca without first uniting with the Wolffian duct. Its opening then lies above that of the Wolffian duct, between it and the opening into the cloaca of the true urinary canal, of which we shall speak directly.

The anterior extremity of the duct of Müller which lies about on a level with the fifth protovertebra is never closed in. Here the original furrow remains open, and forms a funnel-shaped opening into the tube from the pleuroperitoneal cavity. In sections of the sixth day the duct of Müller is to be seen lying between the duct of the Wolffian body and the pleuroperitoneal cavity. Its diameter is generally smaller than that of the Wolffian duct.

19. Between the S0th and 100th hour of incubation, the permanent kidneys begin to make their appearance, and as is the case with the Wolffian bodies, the first portion of them to appear is their duct. Near its posterior extremity the Wolffian duct becomes expanded, and from the expanded portion a diverticulum is constricted off which in a transverse section lies above the original duct, and the blind end of which points forwards, that is, towards the head of the chick. This is the duct of the permanent kidney or ureter. At first the ureter and the Wolffian duct open by a common trunk into the cloaca, but this state of things lasts for a short time only, and by the sixth day the two ducts have independent openings.

The earlier state of things was overlooked by Remak, who thus came to give an incorrect account of the origin of the duct of the kidneys.

Kupffer (Untersuchung über die Entwickelung des Hurn- und Geschlechtssystems, Archiv für Microscop. Anat. Vol. II. I 866) was the first to give a correct account of the development of the duct of the permanent kidneys in the chick. His observations have since been confirmed by a number of other observers, including Waldeyer.

In sections of a somewhat later period the duct of the kidneys can be seen to lie above (dorsal to) the Wolffian duct, and farther from it than the duct of Miiller.

The formation of the kidneys themselves is very similar to the formation of the Wolffian bodies. 
From the upper end of the ureter diverticula are given off at right angles into the intermediate cell-mass. These lengthening and becoming twisted, form the tubuli uriniferi, while the mesoblast around their extremities becomes directly converted into the Malpighian bodies and the capillary network of the kidneys. Corresponding to the relative position of their ducts, the kidney lies above the Wolffian body. At its first appearance it forms an oval body, lying in the upper part of intermediate cell-mass between the Wolffian body and the vertebral column, and is placed rather nearer the median line than the Wolffian body.

The formation of the kidneys takes place before the end of the seventh day, but they do not become of functional importance till considerably later.

From their mode of development it clearly follows that the permanent kidneys are merely parts of the same system as the Wolffian bodies, and that their separation from these is an occurrence of a purely secondary importance.

20. Before describing the subsequent fate of the Wolffian and Müllerian ducts, it will be necessary to give an account of the formation of the true sexual glands, the ovaries and testes.

A.t the first appearance of the projection from the intermediate mass, which we may now call the genital ridge, a columnar character is not only visible in the layer of cells covering the nascent ridge itself along its whole length, but may also be traced for some little distance outwards on either side of the ridge in the cells lining the most median portions of both somatopleure and splanchnopleure. Passing outwards along these layers, the columnar cells gradually give place to a flat tesselated epithelium. As the ridge continues to increase and project, the columnar character becomes more and more restricted to cells covering the ridge itself, in which at the same time it becomes more distinct. On the outer side of the ridge, that is on the side which looks towards the somatopleure, the epithelium undergoes, as we have seen, an involution to form the duct of Mïller, and for some little time retains in the immediate neighbourhood of that duct its columnar character (Fig. 51, $a^{\prime}$ ), though eventually losing it. 
The median portion of the ridge is occupied by the projection of the Wolffian body, and here the epithelium rapidly becomes flattened.

On the inside of the ridge, however, that is on the side looking towards the splanchnopleure, the epithelium not only retains its columnar character, but grows several cells deep (Fig. 51, a), while at the same time the mesoblast $(E)$ underlying it becomes thickened. In this way, owing partly to the increasing thickness of the epithelium, and partly to the accumulation of mesoblast beneath it, a slight eminence is formed, which when viewed from above, after opening the

FIG. 5 I.

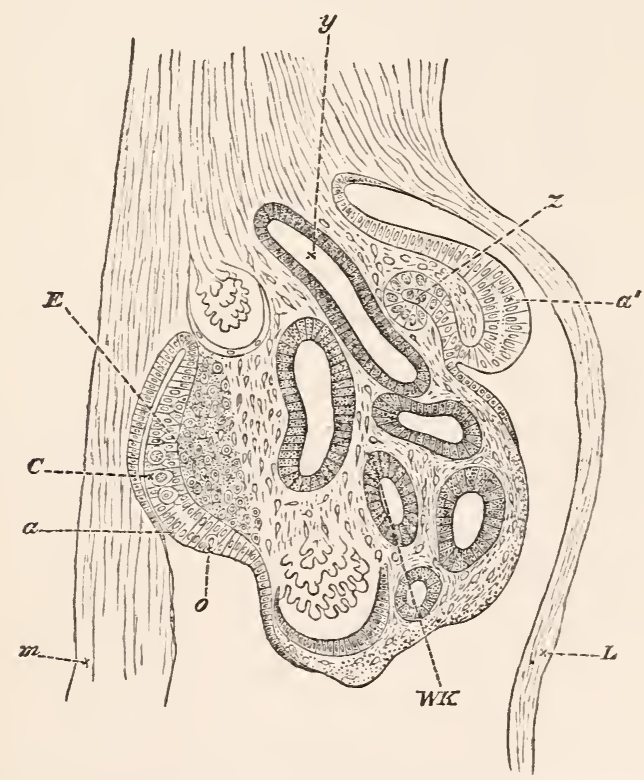

Section of the intermediate Cell-mass on the Fourth Dar. (From Waldeyer.) Magnified 160 times.

$m$. mesentery. $L$. somatopleure. $a^{\prime}$. portion of the germinal epithelium from which the involution to form the duct of Müller $(z)$ takes place. $a$. thickened portion of the germinal epithelium in which the primitive ova $C$ and $o$ are lying. $E$. modified mesoblast which will form the stroma of the ovary. WK. Wolffian body. y. Wolffian duct. 
abdominal cavity, appears in direct light as a fusiform white patch or streak, in its early stages extending along the whole length of the Wolffian body and genital ridge, but subsequently restricted to its anterior portion. Its appearance under these circumstances has been well described by Von Baer.

This 'sexual eminence' is present in the early stages of both sexes. In both the epithelium consists of several layers of short cylindrical cells, a few of which are conspicuous on account of their size and their possessing a highly refractive oval nucleus of considerable bulk; in both, the underlying thickened mesoblast consists-as indeed at this epoch it does generally in all parts of the body-of spindle-shaped cells.

The larger conspicuous cells of the epithelium, which appear to have quite a common origin with their fellow cells, and to rise from them by direct differentiation, and which are seen at the first in male as well as female embryos, are the primordial ova (Fig. 51, o). Thus in quite early stages it is impossible to detect the one sex from the other. At about the 80th to the 100th hour, however, a distinction becomes apparent.

In the males, the epithelium with its underlying mesoblast ceases to develope; the primordial ova neither increase nor multiply. On the contrary, they disappear, and the whole sexual eminence fades away.

In females, on the other hand, the primordial ova enlarge and become more numerous, the whole epithelium growing thicker and more prominent. The spindle-shaped cells of the underlying mesoblast also increase rapidly, and thus form the stroma of the ovary. The growth of this stroma bears subsequently such a relation to that of the epithelium, that the primordial ova appear to sink into the stroma, and each ovum, as it descends, to carry with it a number of the ordinary epithelium-cells, which arrange themselves round it in a distinct layer. In this way each ovum becomes invested by a capsule of vascular connective tissue, lined internally by a layer of epithelium; the whole constituting a Graffian follicle. The large nucleus of the primordial ovum becomes the germinal vesicle, while the ovum itself remains as the true ovum; this subsequently becomes enlarged by the addition of a quantity of yolk derived from the epitholial lining of the follicle. 
Pflüger (Die Eierstocke der Säugethiere u. des Menschen, Leipzig, r863) described the ova as arising, in mammals, out of the epithelium of tubular glands, a chain of several ova being frequently found in one tube and the tube becoming subsequently divided by constrictions into as many follicles. According to Waldeyer however, whose account we have followed above, the primordial ova make their appearance as individual specialized epithelium-cells, without the preformation of any tubular glands, the capsule or Graffian follicle being a later product. Waldeyer's views have been on the whole generally accepted (Leopold, Untersuch. über das Epithel. des Ovariums. Inaug. Diss. Leipzig, I8;0, Romiti, Max Schultze's Archiv, I873, Bd. x.), though opposed by Kapff (Reichert and Du Bois Reymond's Archiv, 1872), and more recently by Sernoff (loc. cit.).

The first traces of the testes are found in the dorsal and inner side of the intermediate cell-mass, and appear about the sixth day. From the first they differ from the rudimentary ovaries, by coming into somewhat close connection with the Wolffian bodies; but occupy about the sane limits from before backwards. The mesoblast in the position we have mentioned begins to become somewhat modified, and by the eighth day is divided by septa of connective tissue into a number of groups of cells; which are the commencing tubuli seminiferi. By the sixteenth day the cells of the tubuli have become larger and acquired a distinctly epithelial character.

Waldeyer is of opinion that the tubules of the Wolffian body penetrate into the tissue from which the testes are formed, and becoming much finer than the remainder of the tubules constitute the 'tubuli seminiferi.' Apart from its inherent difficulties, this view has not been corroborated by any subsequent observer.

It is distinctly denied by Sernoff (loc. cit.), who further states that the testes are entirely formed out of the mesoblast of the intermediate cell-mass, and that their rudiments have no connection either with the germinal epithelium or with the tubules of the Wolffian body.

We have now described the origin of all the parts which form the urinary and sexual systems, both of the embryo and adult. It merely remains to speak briefly of the changes, which on the attainment of the adult condition take place in the parts described.

The Wolffian body, according to Waldeyer, may be said to consist of a sexual and urinary part, which can, he states, be easily distinguished in the just-hatched chick. The sexual part becomes in the cock the after-testes or coni vasculosi, and consists of tubules which lose themselves on the one hand in the seminiferous tubules, and on the other hand, in birds, probably form the whole of what can be called the epididymis. In the hen it forms part of the parovarium of 
His, and is composed of well-developed tubes without pigment. The urinary part forms in both sexes a small rudiment, consisting of blindly ending tubes with yellow pigment, but is most conspicuous in the hen.

The Wolffian duct remains as the vas deferens in the male. In the female it becomes atrophied and nearly disappears.

The duct of Müller on the right side (that on the left side with the corresponding ovary generally disappearing) remains in the female as the oviduct. In the male it is almost entirely obliterated on both sides.

21. We may return to the changes which are taking place in the circulation.

On the fourth day, the point at which the dorsal aorta divides into the two branches which we may now call the iliac arteries is carried much further back towards the tail.

A short way beyond the point of bifurcation, each iliac gives off a branch to the newly formed allantois. It is not, however, till the second half of the fourth day, when the allantois grows rapidly, that these allantoic, or as we may now call them umbilical, arteries acquire any importance, if indeed they are present before. With the increase of the allantois they speedily acquire such a size, that the iliac trunks from which they were given off seem to be mere branches of themselves.

The omphalo-mesaraic arteries are before the end of the day given off from the undivided aortic trunk as a single but quickly bifurcating vessel, the left of the two branches into which it divides being much larger than the right.

During the third day, we saw that the arterial arch running in the first visceral fold became obliterated, the obliteration being accompanied by the appearance of a new (fourth) arch running in the fourth visceral fold on either side.

During the fourth day the second pair of arterial arches also becomes nearly (if not entirely) obliterated; but a new pair of arches is developed in the last (fifth) visceral fold, behind the last visceral cleft; so that there are still three pairs of arterial arches, which however now run in the third, fourth and fifth visceral folds. The last of these is as yet small, and together with the slight remains of the second 
pair of arches we may consider that there are in all four pairs of arches. When the first and second arches are obliterated, it is only the central portion of each arch on either side which absolutely disappears. The ventral portion connected with the bulbus arteriosus, and the dorsal portion which joins the dorsal aorta, both remain, and are both carried straight forward towards the head. The ventral portions of both first and second arches unite on each side to form a branch, the external carotid (Fig. 52, E. CA), which runs straight up from the bulbus arteriosus to the head.

Fig. 52.

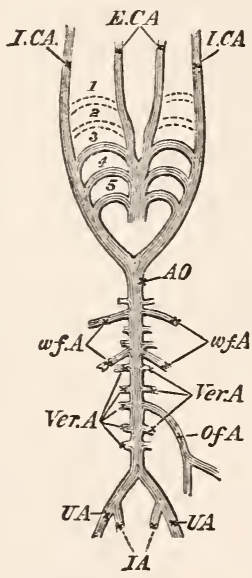

State of Arterial Circulation on the Fifth or Sixth Dar.

E. CA. external carotid. I. CA. internal carotid. AO. dorsal aorta. $u f . A$. arteries to the Wolffian bodies. Ver. $A$. arteries given off between each of the vertebræ. Of. A. omphalo-mesaraic artery. UA. umbilical artery. IA. iliac artery.

In the same way the dorsal portions form a branch, the internal carotid, which takes its origin from the dorsal or far end of the third arch.

22. In the venous system important changes also occur.

As the liver in the course of its formation wraps round the common trunk of the omphalo-mesaraic veins, or meatus venosus, it may be said to divide that vessel into two parts: 
into a part nearer the heart which is called the sinus venosus (Fig. 53, S.V.), and into a part surrounded by the liver which is called the ductus venosus. Beyond, i.e. behind the liver, the ductus venosus is directly continuous with the omphalo-mesaraic veins, or as we may now say, vein, for the right trunk has become so small as to appear a mere branch of the left. (Fig. 53, Of.)

We saw that on the third day the ductus venosus, while running through the liver, exhibited numerous bulgings

Fig. 53.

Diagraj of the Venous Circulation at the Commencement of the Fifth DAY.

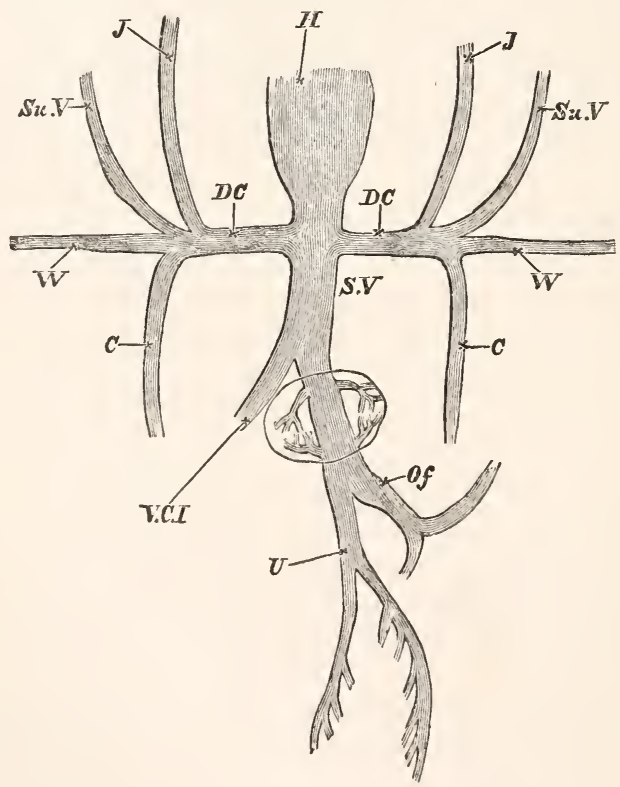

II. heart. D. C. ductus Cuvieri. Into the ductus Cuvieri of each side fall $J$. the jugular vein, or superior cardinal vein, $S u$. $V$. the superior vertebral vein, $W$. the vein from the wing and $C$. the inferior cardinal vein. S. V. sinus venosus. $O f$. omphalo-mesaraic vein. $U$. umbilical vein, which at this stage gives off branches to the body-walls. 
indicative of branches about to be formed. These are on the fourth day actually formed, and become connected with the capillary network simultaneously developed in the hepatic substance in such a way that those branches which come off from the ductus venosus soon after its entrance between the liver-lobes, carry blood into the substance of the liver, while those which join the ductus venosus shortly before it leaves the liver, carry blood away from the hepatic substance into the ductus. The former are called vence advehentes, the latter vence revehentes. As a result of this arrangement, there is a choice of paths for the blood in passing from the omphalomesaraic vein to the sinus venosus; it may pass through the capillary network of the liver, going in by the venæ advehentes, and coming back again by the venæ revehentes, or it may go straight through the ductus venosus without passing at all into the substance of the liver.

As the alimentary canal by its continued closing in becomes on the fourth day more and more distinct from the yolk-sac, it gradually acquires veins of its own, the mesenteric veins, which first appear as small branches of the omphalomesaraic vein, though eventually, owing to the change in the relative size and importance of the yolk-sac and intestine, the latter seems to be a branch of one of the former.

Corresponding to the increase in the size of the head, the superior cardinal veins (Fig. 53, J.) become larger and more important and are joined by the superior vertebral $(S u . V$.) and wing veins $(W)$. As before, they form the ductus Cuvieri (D.C.) by joining with the cardinal veins $(C)$.

The latter are now largely developed; they seem to take origin from the Wolffian bodies, and their size and importance is in direct proportion to the prominence of these bodies. They might be called the veins of the Wolffian bodies.

As the kidneys begin to be formed, a new single median vein makes its appearance, running from them forwards, beneath the vertebral column, to fall into the sinus venosus (Fig. 53, V.C.I.). This is the vena cava inferior.

As the lungs are being formed, the pulmonary veins also make their appearance and become connected with the left side of the auricular division of the heart.

The blood carried to the allantois by the umbilical arteries is brought back by two veins which very soon after 
their appearance unite close to the allantois into a single trunk, the umbilical vein, which, running along the splanchnopleure, falls into the omphalo-mesaraic vein (Fig. 53, $U$ ).

23. Meanwhile the heart is undergoing considerable changes. 'Though the whole organ still exhibits a marked curvature to the right, the ventricular portion becomes directed more distinctly downwards, forming a blunted cone whose apex will eventually become the apex of the adult heart.

The concave (or dorsal) walls of the ventricles become much thicker, as did the convex or ventral walls on the third day.

Well-marked constrictions now separate the ventricles from the bulbus arteriosus on the one hand, and from the auricles on the other. The latter constriction is very distinct, and receives the name of canalis auricularis (Fig. 54, C.A.); the former, sometimes called the fretum Halleri, is far less conspicuous.

The most important event is perhaps the formation of the ventricular septum. This, which commenced on the third day as a crescentic ridge or fold springing from the convex or ventral side of the rounded ventricular portion of the heart, now grows rapidly across the ventricular cavity towards the concave or dorsal side. It thus forms an incomplete longitudinal partition extending from the canalis auricularis to the commencement of the bulbus arteriosus, and dividing the twisted ventricular tube into two somewhat

Fig. 54.

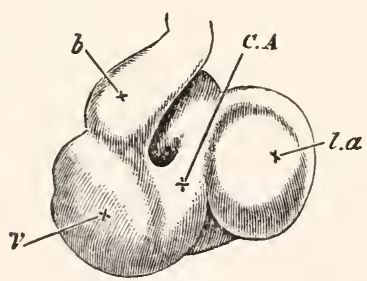

Heart of a Chick on the Fourth Day of Incubation vietwed from the Ventral Surface.

l. a. left auricular appendage. C. A. canalis auricularis. $V$. ventricle. $b$. bulbus arteriosus. 
curved canals, one more to the left and above, the other to the right and below. These communicate freely with each other, above the free edge of the partition, along its whole length.

Externally the ventricular portion as yet shews no division into two parts.

The bulbus arteriosus (Fig. 54, b) has increased in size, and is now very conspicuous.

The venous end of the heart is placed still more dorsal, and to the left of the arterial end; its walls are beginning to become thicker.

The auricles are nearly if not quite as far forward as the ventricles; and the auricular appendages (Fig. 54, l.a.), which were visible even on the third day, are exceedingly prominent, giving a strongly marked external appearance of a division of the auricular portion of the heart into two chambers; but Von Baer was unable to detect at this date any internal auricular septum.

24. The chief events of the fourth day are :-

(1) The increase of the cranial and body flexure.

(2) The increase in the tail-fold.

(3) The formation of the limbs as local thickenings of the Wolffian ridge.

(4) The formation of the olfactory grooves.

(5) The absorption of the partition between the mouth and the throat.

(6) The formation of the allantois as a diverticulum of the alimentary canal.

(7) The formation of the spinal ganglia.

(8) The vacuolation of the cells of the notochord.

(9) The formation of the Wolffian body.

(10) The involution of the germinal epithelium to form the duct of Müller.

(11) The appearance of the primitive ova in the germinal epithelium.

(12) The development of a fifth pair of arterial arches and the obliteration of the second pair.

(13) The origin from the ductus venosus of the capillaries of the liver.

(14) The development of the 'canalis auricularis,' the growth of the septum of the ventricles and of the auricular appendages. 


\section{CHAPTER VII.}

THE CHANGES WHICH TAKE PLACE ON THE FIFTH DAY.

1. ON opening an egg about the middle of the fifth day, the observer's attention is not arrested by any new features; but he notices that the progress of development, which was so rapid during the later half of the fourth day, is being continued with undiminished vigour.

The allantois which on the fourth day began to project from the pleuroperitoneal cavity has grown very rapidly, and now stretches away from the somatic stalk far over the right side of the embryo (which it will be remembered is lying on its left side) in the cavity between the two amniotic folds (Fig. 8, $K$ ). It is very vascular, and already serves as the chief organ of respiration.

The blastoderm has spread over the whole of the yolksac; and the yolk is thus completely enclosed in a bag whose walls, however, are excessively delicate and easily torn. The vascular area extends over about two-thirds of the yolk.

The splanchnic stalk or umbilical duct has now reached its greatest narrowness; it has become a solid cord, and will undergo no further change till near the time of hatching. The space between it and the somatic stalk is still considerable, though the latter is narrower than it was on the fourth day.

2. The embryo remains excessively curved, so much so indeed that the head and the tail are nearly in contact.

The limbs have increased, especially in length; in each a distinction is now apparent between the more cylindrical 
stalk and the flattened terminal expansion; and the cartilaginous precursors of the several bones have already become visible.

The fore and hind limbs are still exceedingly alike, and in both the stalk is already beginning to be bent about its middle to form the elbow and knee respectively.

The angles of both knee and elbow are in the first instance alike directed outwards and somewhat backwards. By the eighth day, however, the elbow has come to look directly backwards and the knee forwards. In consequence of this change, the digits of the fore limb point directly forwards; those of the hind limb directly backwards. This state of things is altered by a subsequent rotation of the hand and foot on the arm and leg, so that by the tenth day the toes are directed straight forwards, and the digits of the wing, backwards and somewhat downwards, the elbow and knee almost touching each other.

While these changes are taking place, the differences between wing and foot become more and more distinct. The cartilages of the digits appear on the fifth day as streaks in the broad flat terminal expansions, from the even curved edge of which they do not project. On the sixth or seventh day the three digits of the wing (the median being the longest), and the four (or in some fowls five) digits of the foot may be distinguished, and on the eighth or ninth day these begin to project from the edge of the expanded foot and wing, the substance of which, thin and more or less transparent, remains for some time as a kind of web between them. By the tenth day, the fore and hind extremities, save for the absence of feathers and nails, are already veritable wings and feet.

At an early period of development we find the following elements in the avian manus, as separate masses of cartilage.

In the carpus there are four elements. Two in the proximal row which remain distinct through life, viz. (I) the radiale, (2) the united intermedium and ulnare. In the distal row (according to some recent observations of Dr Rosenberg, Zeitschrift für Wiss. Zoologie, I873, p. I39, etc.) there are also two elements. One of these is the united first and second carpal which we Inay call carpal I-II, and the other is the united third and fourth carpal which we may call carpal III-Iv. These subsequently unite with the metacarpal bones, and form with them a united 'carpo-metacarpus' comparable with the tarso-metatarsus of the avian foot.

Four metacarpals are present, viz. the first, second, third, and fourth. The 
first, second, and third are the usually recognized elements, and to these Dr Rosenberg's investigations (loc. cit.) have added a fourth. The first, second and third persist in the adult, though they become anchylosed in all recent birds. They also fuse, as we have said above, with the distal row of the carpals. Phalanges belonging to the first, second, and third metacarpals are present.

There thus seem in the avian manus to be no representatives of the centrale, the fifth carpal, the fifth metacarpal and the phalanges of the fourth and fifth digits.

Of the elements we have spoken of in the avian hand, the only ones which require further notice are the carpal ${ }^{\mathrm{I}-\mathrm{II}}$, carpal ${ }^{\mathrm{III}-\mathrm{IV}}$, and the fourth metacarpal.

The united first and second carpal first appears as a small mass of cartilage close to the proximal end of the second metacarpal. In this condition it persists for some time but commences finally to fuse with the first metacarpal; and at a slightly subsequent period with the second metacarpal. These relations with the first and second metacarpals shew without doubt that this little mass of cartilage is the representative of the first and second bones of the distal row of the carpus. In a still later stage carpal ${ }^{1-I I}$ fuses also with carpal $^{\text {III-IV. }}$. Its distinct nature as a separate element in the bird's manus is again shewn during ossification, when there appears for it a separate centre of ossification.

Carpal ${ }^{\text {III-IV }}$ appears about the same time as carpal ${ }^{\mathrm{I}-\mathrm{II}}$ but is at its first appearance united with metacarpals three and four; it soon becomes separated from metacarpal three, and afterwards also from metacarpal four. It subsequently undergoes considerable changes of shape, and rather later fuses with carpal ${ }^{1-11}$. Its true nature is again, as with carpal ${ }^{\mathrm{I}-\mathrm{II}}$ shewn during ossification by the appearance of a separate centre of ossification for it.

The fourth metacarpal is, as we have described, at first united with carpal ${ }^{\mathrm{III}-\mathrm{IV}}$, but subsequently the neck connecting the two becomes constricted, and finally they become completely separated from each other. The small independent mass of cartilage thus formed represents the fourth metacarpal; it applies itself closely to the side of the third metacarpal, though without becoming united with it. It ossifies very late-some time after the hatching of the chick, and after ossification fuses with the third metacarpal-and then in most cases disappears completely.

The pes of a fowl in its early embryonic condition consists of

( I) a mass of cartilage close to the distal end of the tibia. It represents (Gegenbaur) the proximal row of tarsal bones, viz. the 'tibiale,' the 'intermedium,' the 'fibulare,' and the 'centrale.' This cartilage fuses in the adult with the distal end of the tibia.

(2) a mass of cartilage representing the five bones of the distal row of the tarsus. In the adult this unites with the metatarsus, forming a tarsometatarsus.

(3) the metatarsus. There are usually stated to be four metatarsal bones present in the metatarsus of a fowl, which are anchylosed in the adult, but are represented by separate rods of cartilage in the embryo. They are the distal extremity of a first metatarsal, and complete second, third and fourth metatarsal bones. In addition to these Dr Rosenberg (loc, cit.) has found a small oval mass of cartilage representing a fifth metatarsal. Soon after its appearance this becomes fused with the end of the tarsal mass of cartilage representing the fifth tarsal, but later entirely atrophies.

(4) There are four phalanges present both in the embryo and the adult, a number which is never exceeded in birds (except amongst some abnormal breeds of fowls, e.g. the Dorking fowls); though one or more of the four are frequently deficient. 
3. As we mentioned in the last chapter, the formation of the primitive cranium commenced upon the fourth day. This in its earliest stage, inasmuch as it is composed of condensed but otherwise only slightly differentiated mesoblast, may be spoken of as the membranous cranium.

On the sixth day, true hyaline cartilage makes its appearance; and the primitive membranous cranium gives place to the primitive cartilaginous cranium.

The cartilage which is the first to appear, forms a thick plate called the investing mass of Rathke (Fig. 55 , $i v$.), surrounding the whole of that portion of the notochord which projects in front of the foremost protovertebra. The hinder

FIG. 55 .

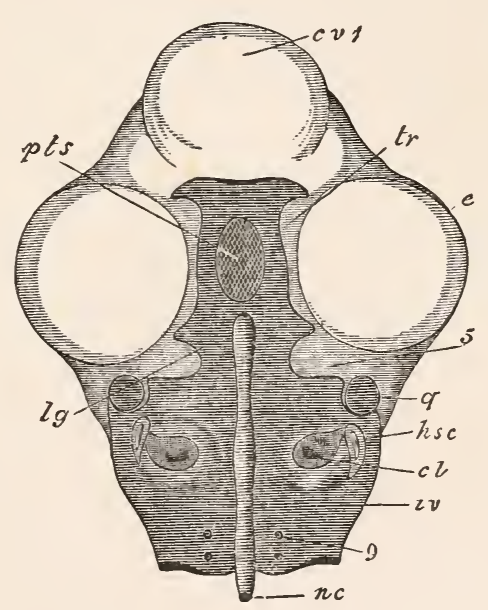

View froy above of the Investing Mass and of the Trabecule on the Fourth Day of Incobation. (From Parker.)

In order to shew this, the whole of the upper portion of the head has been sliced away. The cartilaginous portions of the skull are marked with the dark horizontal shading.

$c v$. I. cerebral vesicles (sliced off). e. eye. nc. notochord. $i v$. investing mass. 9. foramen for the exit of the ninth nerve. cl. cochlea. hsc. horizontal semicircular canal. q. quadrate. 5. notch for the passage of the fifth nerve. $l g$. expanded anterior end of the investing mass. pts. pituitary space. tr. trabeculæ. The reference line $t r$, has been accidentally made to end a little short of the cartilage.

E. 
portion of this investing mass sends upwards along the sides of the brain two lateral projections or wings, which enclose the rudiments of the internal ear. In the chick the portions which thus inclose the auditory sacs seen never to be at any time separate from the remainder of the investing mass. At the front end of the notochord the cartilaginous investing mass divides into two horizontal branches in the form of two cartilaginous rods called the trabeculce (Fig. 55, tr.), which passing forward (in a somewhat different plane from the investing mass), meet again in front, and so enclose a space called the pituitary space $p t s$, into which the infundibulum extends downward. In front of this junction, the trabeculye expand into a somewhat broad plate (subsequently developed into the ethmoid and nasal cartilages), which ends in two horns in the interior of the fronto-nasal process.

The front end of the notochord probably defines the anterior boundary of the basi-occipital. At first it extends quite up to the pituitary space and the starting-point of the trabeculæ. Subsequently, however, there takes place between it and the pituitary space a growth of cartilage in which the ossification for the basi-sphenoid takes place.

The lateral projections at the hinder end of the investing mass grow up behind, and completely enclose that part of the neural canal from which the medulla oblongata is developed, and in it ossifications arise to form the occipital bones and the bones which invest the auditory labyrinth.

It is important to notice that the only segment of the skull, which primarily forms a cartilaginous roof to any part of the brain, is the occipital segment. The roof of the remainder of the skull is formed by membrane-bones.

For the histological differences observable in the development of cartilage and membrane bones, we must refer the reader to treatises on histology; for our purpose it is sufficient to say that a membrane-bone is one which is not preformed in cartilage, while a cartilage-bone is one in which the ossification takes place in a bed of cartilage, which fills the place subisequently occupied by the bone.

The trabeculæ together with the cartilage between the pituitary space and the end of the notochord give rise to the sphenoid bone, while in the cartilage in front of the trabecula the ethmoid and nasal bones are formed. 
From the study of the development of the skull, especially in some of the lower vertebrates, Mr Parker and Professor Huxley have shewn, that the trabeculæ are developed independently of the investing mass, and that their subsequent connection with it is due to a secondary process. Professor Huxley is of opinion that they are to be regarded as the remains of a pair of visceral arches, corresponding with the other tive pairs of arches which we find developed in the chick. The stage in which they exist as simple visceral arches with a core of undifferentiated mesoblast is not seen in the chick. 'i'hey first attract notice when they become cartilaginous rods.

The ordinary visceral arches are, as we have seen, sufficiently obvious, while as yet their mesoblast is quite undifferentiated; but in them, as in the trabeculæe, rods of cartilage are subsequently developed and begin to make their appearance about the fifth day.

The first arch, it will be remembered, budded off a process called the superior maxillary process. The whole arch, therefore, comes to consist of two parts, viz. a superior and an inferior maxillary process; in each of these, cartilaginous rods are developed. In the superior maxillary process, the rod does not appear till the fifth day. It is called from its subsequent fate, the pterygo-palatine rod, and consists of a pterygoid and of a palatine part. In the inferior maxillary process two developments of cartilage take place; one which forms the quadrate in the upper or proximal portion close to the origin of the superior maxillary process, a second in the lower or distal portion, which goes by the name of Meckel's cartilage.

Cartilaginous rods are also formed in the second and third arches. These, which give rise to the hyoids and branchials respectively, quickly come to lie within the first arch, but do not forin a conspicuous portion of the skeleton of the face.

4. Closely connected with the development of the skull. is the formation of the parts of the face.

After the appearance of the nasal grooves, on the fourth day the inouth (Fig. 56 M.) appears as a deep depressiou inclosed by five processes. Its lower border is entirely formed by the two inferior maxillary processes (Fig. $56, F .1$ ), at its sides lie the two superior maxillary processes $S . M$, while above it is bounded by the fronto-nasal process $n f$.

After a while the outer angles of the frontu-nasal process, enclosing the termination of the ethmovomerine plate, project somewhat outwards on each side, giving the end of the 
FIG. 56.
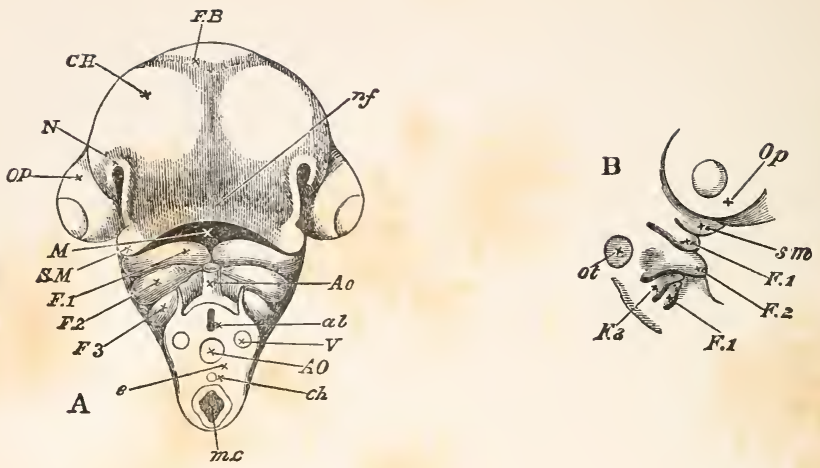

A. Head of an Embryo Chick of the Fodrth Day viewed from below AS AN OPAQUE OBJECT. (Chromic acid preparation.)

$C I I$. cerebral hemispheres. FB. vesicle of the third ventricle. $O p$. eyeball. $n f$. naso-frontal process. $M$. cavity of mouth. S. M. superior maxillary process of $F$. I, the first visceral fold (inferior maxillary process). $F .2, F .3$, second and third visceral folds. $N$. nasal pit.

In order to gain the view here given the neck was cut across between the third and fourth visceral folds. In the section $e$ thus made, are seen the alimentary canal $a l$ with its collapsed walls, the neural canal m.c., the notochord $c h$., the dorsal aorta $A O$, and the vertebral veins $V$.

The incision has been carried just below the upper limit of the pleuroperitoneal cavity, consequently a portion of the somatopleure appears at the angle between the two third visceral folds. Almost embraced by the piece of somatopleure is seen the end of the bulbus arteriosus $A o$.

In the drawing the nasal groove has been rather exaggerated in its upper part. On the other hand the lower part of the groove, where it runs between the superior maxillary process $S . M$. and the broad naso-frontal process, was in this particular embryo extremely shallow and indeed hardly visible. Hence the end of the superior maxillary process seems to join the inner and not, as described in the text, the outer margin of the nasal groove. A few hours later the separation of the two would have been very visible.

B. The same seen sideways, to shew the visceral folds. Letters as before.

process a rather bilobed appearance. These projecting portions of the fronto-nasal process form on each side the inner margins of the rapidly deepening nasal grooves, and are sometimes spoken of as the inner nasal processes. The outer margin of each nasal groove is raised up into a projection frequently spoken of as the outer nasal process which runs downwards to join the superior maxillary process, from which, 
however, it is separated by a shallow depression. This depression, which runs nearly horizontally outwards towards the eyeball, is, according to Coste and Kölliker, subsequently converted into the lachrymal duct.

On the fifth day, the inner nasal processes or lower and outer corners of the fronto-nasal process arching over, unite on each side with the superior maxillary processes. (Compare Fig. 57, which, however, is a view of the head of a chick of the sixth day.) In this way each nasal groove is converted into a canal, which leads from the nasal pit above, into the cavity of the mouth below, and places the two in direct communication. This canal, whose lining consists of epiblast, is the rudiment of the nasal labyrinth.

FIG. 57.

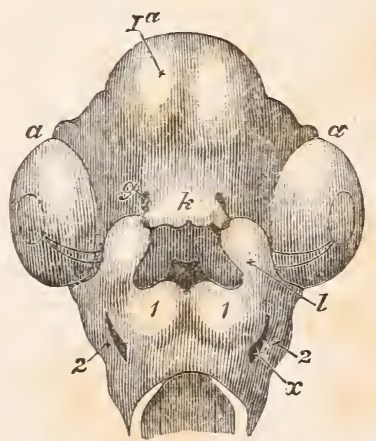

Head of a Chick at the Sixth Day froy below. (Copied from Huxley's Elements of Comparative Anatomy.)

Ia. cerebral vesicles. $a$. eye, in which the remains of the choroid slit can still be seen. $g$. nasal pits. $k$. fronto-nasal process. $l$. superior maxillary process. I. inferior maxillary process or first visceral arch. 2. second visceral arch. $x$. first visceral cleft between the first and second visceral arches.

The cavity of the mouth is seen enclosed by the fronto-nasal process, the superior maxillary processes and the first pair of visceral arches. At the back of it is seen the opening leading into the throat. The nasal grooves leading from the nasal pits to the mouth are already closed over and converted into canals.

By the seventh day (Fig. $\check{8}$ ), not only is the union of the superior maxillary and fronto-nasal processes completed, and the upper boundary of the mouth thus definitely con- 
stituted, but these parts begin to grow rapidly forward, thus deepening the mouth, and giving rise to the appearance of a nose or beak (Fig. 58), which, though yet blunt, is still distinct. The whole of the lower boundary of the buccal cavity is formed by the inferior maxillary processes.

FIG. 58 .

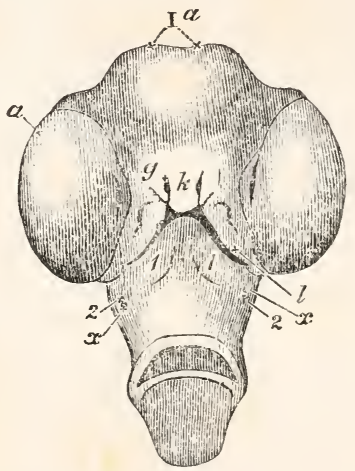

Head of a Chick of the Seventh Day from beiow. (Copied from Huxley's Elements of Comparatize Anatomy.)

I $a$. cerebral vesicles. $a$. eye. $g$. nasal pits. $k$. fronto-nasal process. $l$. superior maxillary process. I. first visceral arch. 2. second visceral arch. $x$. first visceral cleft.

The external opening of the mouth has become much constricted, but it is still enclosed by the fronto-nasal process and superior maxilary processes above, and by the inferior maxillary process (first pair of visceral arclies) below.

The superior maxillary processes have united with the fronto-nasal process, along the whole length of the latter, with the exception of a small space in front, where a narrow angular opening is left between the two.

As we have before mentioned, the ethmovomerine cartilage is developed in the fronto-nasal process, the pterygopalatine bar in the superior maxillary process, Meckel's rartilage and the quadrate in the inferior maxillary process; the other bones which form the boundaries of the mouth in the adult are developed later after all external trace of these parts as separate processes has disappeared. For an account of their formation, however, we must refer the reader to the chapter upon the development of the skull.

At first the mouth is a simple cavity into which the nasal canals open directly. When however the various 
processes unite together to form the upper boundary of the mouth, each superior maxillary process sends inwards a lateral bud. These buds become flattened and form horizontal plates which stretch more and more inward towards the middle line. There they finally meet, and by their union, which is effected first in front, and thence extends backwards, they constitute a horizontal plate, stretching right across the mouth, and dividing it into two cavities-an upper and a lower one.

In the front of the mouth their union is quite complete, so that here there is no communication between the two cavities. Behind, however, the partition is not a complete one; so that the two divisions of the buccal cavity communicate at the back of the mouth. The external opening of the mouth passes into the lower of these two cavities, which may therefore be called the mouth proper. Into the upper chamber the nasal ducts open; it may be called the respiratory chamber and forms the commencement of the chamber of the nose. In birds generally the upper nasal cavity becomes subsequently divided by a median partition into two chambers, which communicate with the back of the mouth by separate apertures. The original openings of the nasal pits remain as the nostrils.

5. One important occurrence of the fifth day is the appearance of the anus, which is formed very much in the same way as the mouth.

Beneath the tail an involution of the epiblast takes place towards the cloaca. At this point the wall of the cloaca, which has here taken no share in the cleavage of the mesoblast, becomes thinner, and is finally perforated. An orifice thus places the cloaca in communication with the exterior, and constitutes the anus.

6. On this day also important changes take place in the spinal cord; and a brief history of the development of this organ may fitly be introduced here.

At the beginning of the third day, the cavity of the neural canal (Fig. 41) is still of considerable width, and when examined in vertical section its sides may be seen to be nearly parallel, though perhaps approximating to each other more below than above.

We say below and above, because a vertical section is naturally examined with its dorsal side uppermost. In the ordinary terminology of the spinal cord, 
above would be posterior and below anterior. These latter terms it will be henceforward most convenient to adopt.

The exact shape, however, varies according to the region of the body from which the section is taken.

The epiblast walls are at this time composed of radiately arranged columnar cells. The cells are much elongated, but somewhat irregular; and it is very difficult in sections to make out their individual boundaries. They contain granular oval nuclei in which a nucleolus can almost always be seen. The walls of the canal are both anteriorly and posteriorly considerably thinner than in the middle.

Towards the end of the third day, changes take place in the shape of the cavity. In the lumbar region its vertical section becomes more elongated, and at the same time very narrow in the middle while expanded at each end into a somewhat bulbous enlargement, producing an hour-glass appearance (Fig. 44). Its walls however still preserve the same histological characters as before.

On the fourth day (Fig. 47) coincidently with the appearance of the spinal nerves, important changes may be observed in the hitherto undifferentiated epiblastic walls.

In the anterior region of the cord, the external portions of the epiblast become modified into grey matter, forming an anterior grey colum which in turn is covered superficially by a mass of white matter forming an anterior white column. The internal portions of the epiblast remain as the epithelium lining the spinal canal. Both columns are formed at the point of entrance of the anterior nerve-roots; and these may easily be traced through the white into the grey matter.

The grey column is composed of numerous small nuclei, each of which appears to be surrounded by a definite mass of protoplasm, though the boundaries of the protoplasm belonging to each nucleus can only occasionally be made out. The nuclei lie in the meshes of a network of fibres continuous with the fibres of the nerve-root, and passing through the mass of grey matter in two directions:-(1) round the anterior end of the spinal canal, immediately outside its epithelium and so to the grey matter on the opposite side, forming in this way an anterior commissure through which a decussation of the fibres from the opposite sides is effected: (2) upwards along the outside of the lateral walls of the canal. 
The posterior roots of the spinal nerves enter the cord near its posterior surface, and at this point the posterior grey columns are formed in a similar way to the anterior. In some cases also the rudiment of a posterior white column may be seen at the junction of the nerve with the epiblast of the canal. The fibres of the posterior root cannot be traced so far into the cord as those of the anterior root.

The grey matter of the cord seems undoubtedly to be formed by a metamorphosis of the external cells of the epiblast of the neural tube, and is directly continuous with the epithelium; there being no strong line of demarcation between them. Whether the fibres which traverse it, and which seem to be partly nervous and partly connective tissue in their nature, are derived from mesoblast or epiblast our observations have not enabled us to determine.

The white matter which caps the grey mass, and which forms the commencement of the anterior white column, is a peculiar tissue. It consists of a network of fibres somewhat resembling the connective tissue network of the white matter of the adult cord, to which it has a further likeness in not being easily stained by carmine. The fibres of which it is composed have a general tendency to be disposed in radiating septa, a peculiarity which is especially noticeable with low powers. Along the fibres and more especially in the septa, numerous highly refracting granules are embedded, and in the meshes pale spherical nuclei with nucleoli are to be seen. The boundary between the white and grey matter is very sharply defined, and we have always failed to trace the fibres of which we are speaking in to the fibres present in the grey matter, though Lockhart Clarke (Phil. Trans. I 862) asserts that they are continuous. Nor can the fibres of the nerve-roots be seen to come into connection with these same fibres. It has generally been assumed that the white matter like the grey is derived from the epiblast: this does not however appear ever to have been clearly proved, while the peculiarities of the tissue, and the fact that it first appears at the origin of the spinal nerves, might seem to indicate that it is directly derived from the mesoblast surrounding the cord; a view which we are inclined to accept.

On the fourth day there is no trace of either an anterior or a posterior fissure, and in the lumbar region the shape of the spinal canal is not very different from what it was on the third day. It appears in sections as a narrow slit dilated somewhat at either end (Fig. 47). The epithelium surrounding the slit is still very thin, especially above and below, but at the anterior end forms a somewhat arched projection with the convex surface turned downwards.

On the fifth and sixth days important changes take place.

By the great increase of the grey matter, which now comes to form the chief mass of the cord, the epithelium is reduced to a thin layer of cells immediately surrounding the canal.

In the dorsal region, the side walls of the laterally com- 
pressed canal come into absolute contact in the middle. So that sections no longer shew an hour-glass cavity, but two more or less elliptical cavities, representing the former terminal enlargements, one anterior and one posterior, separated by a neck in which the epithelium of the one sille is closely applied to that of the other. In other words, the original single canal has been divided longitudinally into an anterior and posterior canal. Of these the anterior will alone remain as the permanent central canal of the spinal cord. In the lumbar region this division has as yet not taken place.

The anterior white columns have very much increased in quantity; the posterior white columns have also become distinct, and the two form together a thick covering for the grey matter. The two columns of each side are continuous with each other, but their line of junction is clearly marked; and on the sixth day there may be seen at this spot a small mass of white matter, differing somewhat from the rest in appearance, which perhaps may be looked upon as the first commencement of the lateral column. The columns of the one side are not continuous with those of the other either posteriorly or anteriorly. In other words, there are as yet no white commissures.

'The anterior ends of the cord on each side of the middle line have commenced to grow downwards. These outgrowths, in which both the white and the grey matter take part, have an important function. They enclose between them a somewhat linear space: the commencement of the anterior fissure. This, which is at first not very deep and rather wide, may be noticed alrearly on the fifth day (L. Clarke) and on the sixth day is very clearly marked.

Corresponding with these grosser changes, certain histological features make their appearance. Between the anterior and posterior parts into which the grey matter is divided on each side, or, as we may now call them, the anterior and posterior cornua, there is found a rather lighter hand of grey matter in which the nuclei are somewhat more scattered. 'The anterior cornu exhibits a further division into an outer and upper part, and a lower and inner part, in both of which the nuclei are more numerous than in the intervening mass. The posterior cornu is of considerably darker colour than the anterior, the difference being due to 
the greater number of nuclei present in the former. The outlines of the cells are more clearly marked and somewhat more angular in shape than they were on the fourth day.

The distinctions between the several parts of the grey matter are chiefly brought about by variations in the number of nuclei in a given area. Throughout the cord fibres of the grey matter seen to be continuous with the epithelium of the neural canal, but this is much more strongiy marked in the posterior than in the anterior region. In the posterior region also, it is still much more difficult to trace the routs of the nerves than in the anterior.

Of the three columns into which the white matter on each side is divided, the anterior column differs from the posterior in being thicker and also in having wider meshes and fewer granules. The lateral column is the most granular of all and very conspicuous. The minute structure of the white matter remains about the same as on the fourth day.

Meanwhile an alteration is taking place in the external outline of the cord. From being, as on the fourth and fitth days, oval in section, it becomes, chiefly through the increase of the white matter, much more nearly circular.

On the seventh day the most important event is the formation of the posterior fissure.

This is brought about by the absorption of the roof of the posterior of the two parts into which the neural canal has become divided.

Between the posterior horns of the cord, the epithelium forming the roof of the, so to speak, posterior canal is along the middle line covered neither by grey nor by white matter, and on the seventh day is partially absorbed, thus transforming the canal into a wedge-shaped fissure, whose mouth however is seen in section to be partially closed by a triangular clump of elongated cells (Fig. 59 c.). Below this mass of cells the fissure is open. It is separated from the 'true spinal canal' by a very narrow space along which the side walls have coalesced. In the lumbar and sacral regions the two still communicate.

We thus find, as was first pointed out by Lockhart Clarke, that the anterior and posterior fissures of the spinal cord are, morphologically speaking, entirely different. The anterior fissure is merely the space left between two lateral downward growths of the cord, while the posterior fissure is part of the original neural canal separated from the rest of 
Fig. 59.

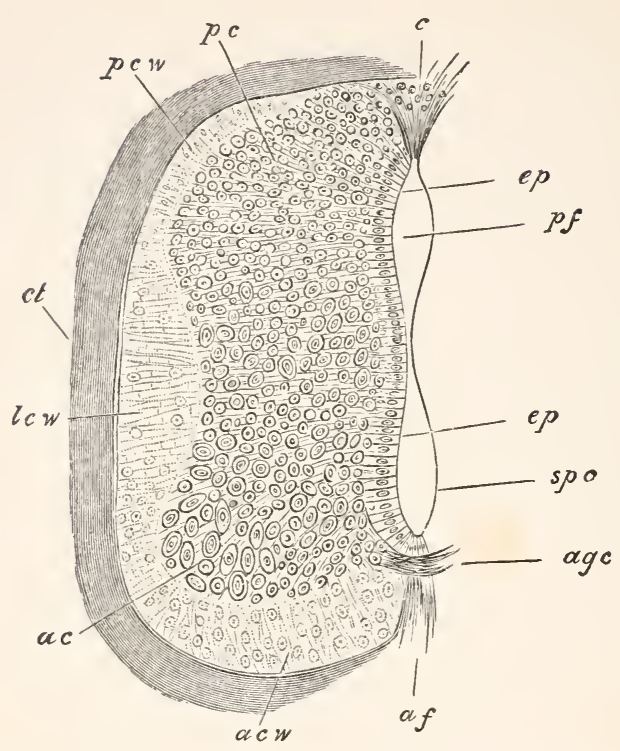

Section throdgh the Spinal Cord of a Seven Days' Chick.

p.c. $w$. posterior white column. l.c.w. lateral white column. a.c.w. anterior white column. $p c$. posterior cornu, of grey matter, consisting of very small cells. a. $c$. anterior cornu of grey matter, with a peculiar mass of very large cells. ep. epithelium lining the original medullary canal. $p . f$. posterior fissure. The posterior fissure is chiefly formed by the upper portion of the original medullary canal which becomes open above. The upper portion of it is now filled with tissue $(c)$ which is probably derived from the epithelium of the medullary canal. The lower portion of the medullary canal becomes the spinal canal $(s p . c$.$) and is eventually entirely shut off$ from posterior fissure. The communication between the spinal canal and the posterior fissure is already narrowed, and if the section had been made further forwards, the two would have been entirely separated from each other.

a. $f$. anterior fissure. This is formed in an entirely different manner from the posterior fissure. It is produced by the anterior column of white, and the anterior cornu of grey matter, growing downwards and leaving between them a fissure. It is at this time filled up with connective tissue.

a.g. $c$. anterior grey commissure. $c$. tissue filling up the end of the posterior fissure. sp. $c$. spinal canal. Only the right half of the cord is representer in the figure. The section passes through the cord between the entrance of two spinal nerves. The angular form of the cells of the cord has not been done justice to by the engraver. 
the cavity (which goes to form the true spinal canal) by a median coalescence of the side walls.

The lateral white columns have on the seventh day increased in size and become less granular, and the lines of junction between them and the anterior columns have now to be arbitrarily selected. The posterior white columns are still much thinner and more granular than the anterior. The nuclei of the white matter are more numerous than before.

Some of the septa of the white matter can now be traced in the one direction into the grey matter, and in the other direction into the connective tissue around the cord. Whether these are nerve-fibres which have separated from the remainder of the fibres, to enter the cord at a different point, or are merely trabeculæ of connective tissue, cannot be absolutely determined. The latter view however seems most probable. In the grey matter, the anterior and posterior divisions are better distinguished than at an earlier date. In particular the nuclei of the cells of the posterior division are both smaller and more numerous than those of the anterior. Some of the fibres from the posterior root, after entering the grey matter, quickly pass out again into the posterior column of the white matter.

In the anterior division of the grey matter, near the entrance of the anterior roots, there is a peculiar and well-marked mass of somewhat triangular cells, with large and distinct nuclei, more deeply stained with carmine than the remainder of the grey matter. This mass of cells is present in the lumbar and sacral regions, but is deficient or very inconspicuous in the dorsal portion of the cord. The nuclei of the whole anterior region of the grey matter have increased in size, and the cells to which they belong (when clearly visible) are usually found to be angular.

Around the true spinal canal, the line of separation between the epithelium and the grey matter is sharply defined, but elsewhere is very indistinct.

By the end of the seventh day, the following important parts of the cord have been definitely established:

(1) The anterior and posterior fissures.

(2) The anterior and posterior horns of grey matter.

(3) The anterior, posterior and lateral columns of white matter.

\section{(4) The spinal canal.}

As yet, however, the grey mass of the two sides of the cord only communicate by the anterior grey commissure, and the white columns of opposite sides do not communicate at all. The grey matter, moreover, still far preponderates over the white matter in quantity.

By the ninth day the posterior fissure is fully formed, and the posterior grey commissure has also appeared.

In the centre of the sacral enlargement this commissure is absent, and the posterior columns at a later period separate 
widely and form the 'sinus rhomboidalis,' which is not, as has been sometimes stated, the remains of the primitive 'sinus rhomboidalis' visible during the second day.

The anterior white columns have much increased on this day, and now form the sides of the already deep anterior fissure. The anterior white commissure does not however appear till somewhat later.

7. The fifth day may perhaps be taken as marking a most important epoch in the history of the heart. The changes which take place on that and on the sixth day, added to those previously undergone, transform the simple tube of the early days of incubation into an almost completely formed heart.

The venous end of the heart, though still lying somewhat to the left and above, is now placed as far forwards as the arterial end. the whole organ appearing to be drawn together. The ventricular septum is complete.

The apex of the ventricles becomes more and more pointed. In the auricular portion a small longitudinal fold appears as the rudiment of the auricular septum, while in the canalis auricularis, which is now at its greatest length, there is also to be seen a commencing transverse partition tending to separate the cavity of the auricles from those of the ventricles.

About the 106th hour, a septum begins to make its appearance in the bulbus arteriosus in the form of a longitudinal fold, which according to Dr Tonge (Proc. of Royal Soc. 1868 ) starts, not (as Von Baer thought) at the end of the bulbus nearest to, but at that furthest removed from, the heart. It takes origin from the wall of the bulbus between the fifth and fourth pairs of arches and grows downwards in such a manner as to divide the bulbus into two channels, one of which leads from the heart to the fourth and third pair of arches and the other to the fifth pair. The free edge of the septum is somewhat V-shaped, so that its two legs as it were project downwards towards the heart, further than its central portion; and this shape of the free edge is maintained duriug the whole period of its growth. Its course downwards is not straight but spiral, and thus the two channels into which it divides the bulbus arteriosus, wind spirally the one uver the other. The existence of the septum can only be 
ascertained at this stage by dissection or by sections, there being as yet no external signs of the division.

At the time when the septum is first formed, the opening of the bulbus arteriosus into the ventricles is narrow or slitlike, apparently in order to prevent the flow of the blood back into the heart. Soon after the appearance of the septum, however, semilunar valves (Tonge, loc. cit.) are developed from the wall of that portion of the bulbus which lies between the free edge of the septum and the cavity of the ventricles.

These arise as six solid outgrowths of the wall arranged in pairs, an anterior, an inner, and an outer pair, one valve of each pair belonging to the one and the other to the other of the two main divisions of the bulbus which are now being established.

The anterior and the inner pairs of valves are the first to appear: the former as two small sulid prominences separated from each other by a narrow groove; the latter as a single shallow ridge, in the centre of which is a prominence indicating the point where the ridge will subsequently become divided into two. The outer pair of valves appear opposite each other, at a considerably later period, between the ends of the other pair of valves on each side.

As the septum grows downwards towards the heart, it finally reaches the position of these valves. One of its legs then passes between the two anterior pair of valves, and the other unites with the prominence on the inner valve-ridgre. At the same time the growth of all the parts causes the valves to appear to approach the heart and thus to be placed quite at the top of the ventricular cavities. The free edge of the septum of the bulbus now fuses with the ventricular septum, and thus the division of the bulbus into two separate channels, each provided with three valves, and each communicating with a separate side of the heart, is complete, the position of the valves not being very different from what it is in the adult heart.

That division of the bulbus which opens into the fifth pair of arches is the one which communicates with the right ventricle, while that which opens into the third and fourth pairs communicates with the left ventricle. The former becomes the pulmonary artery, the latter the commencement of the systemic aorta. 
The external constriction actually dividing the bulbus into two vessels, does not begin to appear till the septum has extended some way back towards the heart.

The semilunar valves become pocketed at a period considerably later than their first formation (from the 147th to the 165th hour) in the order of their appearance.

8. Towards the end of the fifth and in the course of the sixth day further important changes take place in the heart.

The venous end with its two very conspicuous auricular appendages, comes to be situated more above (dorsal to) the arterial end, though it still turns rather towards the left. The venous portion of the heart undergoes on the sixth day or even near to the end of the fifth, such a development of the muscular fibres of its walls, that the canalis auricularis becomes almost entirely concealed. The point of the heart is now FIG. 60.
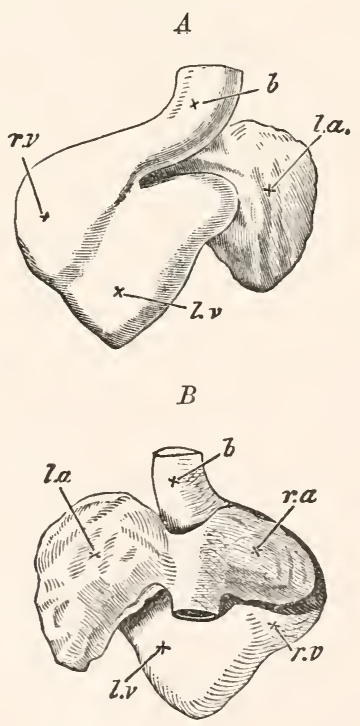

Two views of the Heart of a Chick upon the Fifth Day of Incobation, $A$ from the ventral, $B$ from the dorsal side.

l.a. left auricular appendage, $r . a$. right auricular appendage, $r . v$. right ventricle. $l . v$. left ventricle. $b$. bulbus arteriosus. 
directed nearly backwards (i.e. towards the tail), but also a little downwards.

An alteration takes place during the sixth day in the relative position of the parts of the ventricular division of the heart. The right ventricle is now turned towards the abdomiual surface, and also winds to a certain extent round the left ventricle. It will be remembered that on the fourth day the right ventricle was placed above (dorsal to) the left.

The right ventricle is now also the smaller of the two, and the constriction which divides it from the left ventricle does not extend to the apex of the heart (Fig. 60). It has, however, a very marked bulge towards the right.

At first the bulbus arteriosus appeared to come off chiefly from the left ventricle; during the fifth day, and still more on the sixth, it appears to come from the right chamber. This is caused by the canal from the right ventricle into the bulbus arteriosus passing towards the left, and on the ventral side, so as entirely to conceal the origin of the canal from the left chamber of the heart. On the seventh day the bulbus arteriosus appears to come less markedly from the right side of the heart.

All these changes, however, of position of the bulbus arteriosus only affect it externally; during the whole time the two chambers of the heart open respectively into the two divisions of the bulbus arteriosus. The swelling of the

Fig. 6r.

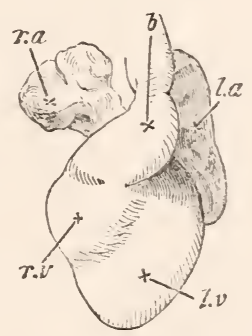

heskt of a Chick upon the Sixth Dat of Ixcubation, frosi the Ventral Surface.

l. $a$. left auricular appendage, $r$. $a$. right auricular appendage, $r . v$.right ventricle, $l . v$. left ventricle, $b$. bulbus arteriosus. 
bulbus is much less marked on the seventh day than it was before.

At the end of the sixth day, and even on the fifth day (Figs. 60, 61), the appearance of the heart itself, without reference to the vessels which come from it, is not very dissimilar from that which it presents when adult.

The original curvature to the right now forms the apex of the ventricles, and the two auricular appendages are placed at the anterior extremity of the heart.

The most noticeable difference (in the ventral view) is the still externally undivided condition of the bulbus arteriosus.

About the sixth or, perhaps, even on the fifth day, the pericardium, according to Von Baer, makes its appearance. Its mode of formation is not exactly known, but it probably takes origin from folds of the lining of the thoracic cavity which meet and coalesce.

9. The subsequent changes which the heart undergoes are concerned more with its internal structure than with its external shape. Indeed, during the next three days, viz. the eighth, ninth, and tenth, the external form of the heart remains nearly unaltered.

In the auricular portion however, the septum which commenced on the fifth day becomes now more conspicuous. It is placed vertically, and arises from the ventral wall; commencing at the canalis auricularis and proceeding backwards, it does not as yet reach the opening into the sinus venosus.

The blood from the sinus, or, as we may call it, the inferior vena cava, enters the heart obliquely from the right, so that it has a tendency to flow towards the left auricle of the heart, which is at this time the larger of the two.

The valves between the ventricles and auricles are now well developed, and it is about this time that the division of the bulbus arteriosus into the aorta and pulmonary artery becomes visible on the exterior.

By the eleventh or thirteenth day the right auricle has become as large as the left, and the auricular septum much more complete, though there is still a small opening, the foramen ovale, by which the two cavities communicate with each other. Through this foramen the greater part of the blood of the vena cava inferior, which is now joined just 
at its entrance into the heart by the right vena cava superior, is directed into the left auricle. The left vena cava superior enters the right auricle independently; between it and the inferior vena cava is a small valve which directs its blood entirely into the right auricle.

On the sixteenth day the right vena cava superior, when viewed from the exterior, still appears to join the inferior vena cava before entering the heart; from the interior however the two can now be seen to be separated by a valve. This valve, called the 'Eustachian valve,' extends to the opening of the left vena cava superior, and into it the valve which in the earlier stage separated the left superior and inferior venæ cavæ has apparently become merged. There is also on the left side of the opening of the inferior cava a membrane, stretching over the foramen ovale, and serving as a valve for that orifice. The blood from the inferior cava still passes chiefly into the left auricle through the foramen ovale; while the blood from the other two venæ cavæ now falls into the right auricle, being prevented from entering the left chamber by the Eustachian valve.

Hence, since at this period also the blood from the left ventricle passes to a great extent to the anterior portion of the body, there is a species of double-circulation going on. The greater part of the blood from the allantois entering the left auricle from the inferior vena cava passes into the left ventricle and is thence sent chiefly to the head and anterior extremities; from these it is brought back through the right auricle to the right ventricle, from whence it is returned along the aorta to the allantois.

From the seventeenth to the nineteenth day, the right auricle becomes larger than the left. The large Eustachian valve still prevents the blood from the superior cavæ from entering the left auricle, while it conducts the blood from the inferior vena cava into that chamber through the foramen ovale. The entrance of the inferior vena cava is however further removed than it was from the foramen ovale, and the increased flow of blood from the lungs prevents all the blood of the inferior cava from entering into the left auricle. At the same time the valve of the foramen ovale prevents the blood in the left auricle from entering the right auricle.

During the period from the seventh day onwards, the 
apex of the heart becomes more marked; the arterial roots are more entirely separated and the various septa completed, so that when the foramen ovale is closed and the blood of the inferior vena cava thereby entirely confined to the richt auricle, the heart has practically acquired its adult condition.

10. The fifth day may also be taken as marking the epoch at which histological differentiation first becomes distinctly established.

It is of course true that long before this date, even from the earliest hours, the cells in each of the three fundamental lavers have ceased to be everywhere alike. Nevertheless the changesundergone by the several cells have been few and slight. The cells of epiblastic origin, both those going to form the epidermis and those included in the neural involution, are up to this time simple more or less columnar cells; they may be seen here elongated, there oval, and in another spot spheroidal; here closely packed with scanty protoplasm, there scattered with each nucleus well surrounded by cell-substance; but wherever they are found they may still be recognized as cells of a listinctly epithelial character. So also with the cells of hypoblastic origin, whether simply lining the alimentary canal or taking part in the formation of the compound glands. Even in the mesoblast, which undergoes far more changes than either of the other layers, not only increasing more rapidly in bulk but also serving as the mother tissue for a far greater number of organs, the alterations in the individual cells are, till near upon the fifth day, insignificant. Up to this time, the mesoblast may be spoken of as consisting of little more than indifferent tissue:-of nuclei imbedded in a protoplasmic cell-substance. In one spot the nuclei are closely packed together, and the cell-substance scanty and compact; at another the nuclei are scattered about with spindle-shaped masses of protoplasm attached to each, and there is a large development either of intercellular spaces or of intracellular vacuoles, filled with clear fluid. The protoplasm differs in various places, chiefly in being more or less granular, and less or more transparent, having as yet undergone but slight chemical transformation. Up to this epoch (with the exception of the early differentiated blood), there are no distinct tissues, and the rudiments of the various 
organs are simply marked out by greater or less condensation of the simple mesoblastic substance.

From the fifth day onwards, however, histological differentiation takes place rapidly; and it soon becomes possible to speak of this or that part as being composed of muscular, or cartilaginous, or connective \&c. tissue. It is not within the scope of the present work to treat in detail of these histogenetic changes, for information concerning which we would refer the reader to histological treatises. We have alręady had occasion to refer incidentally to many of the earliest histological events; and shall content ourselves by giving a brief summary of the derivation of the tissues of the adult animal from the three primary layers of the blastoderm.

The epiblast or upper layer of many embryologists forms primarily two very important parts of the kody, viz. the central nervous system and the epidermis.

It is from the involuted epiblast of the neural tube, that the whole of the grey matter of the brain and spinal cord appears to be developed, the simple columnar cells of the epiblast being apparently directly transformed into the characteristic caudate nerve-cells. There is, however, some doubt whether mesoblast cells may not possibly enter into its formation, and it is very probable that the white matter of the brain and spinal cord is derived from the mesoblast alone.

The epithelium (ciliated in the young animal) lining the canalis centralis of the spinal cord, together with that lining the ventricles of the brain, all which cavities and canals are, as we have seen, derivatives of the primary neural canal, is the undifferentiated remnant of the primitive epiblast.

The epiblast as we have said also forms the epidermis; not however the dermis, which is of mesoblastic origin. The line of junction between the epiblast and the mesoblast coincides with that between the epidermis and the dermis. From the epiblast are formed all such tegumentary organs or parts of organs as are epidermic in nature.

In addition to these, the epiblast plays an important part in the formation of the organs of special sense.

According to their mode of formation, these organs may be arranged into two divisions. In the first come the cases where the sensory expansion of the organ of special 
sense is derived from the involuted epiblast of the medullary canal. To this class belongs the Retina, including the epithelial pigment of the choroid, which is formed from the original optic vesicle budded out from the fore-brain.

To the second class belong the epithelial expansions of the membranous labyrinth of the ear, and the cavity of the nose, which are formed by involution from the superficial epiblast covering the external surface of the embryo. These accordingly have no primary connection with the brain. We may also fairly suppose that the 'taste bulbs' and the nervous cells which have lately been described as present in the epidermis are also structures formed from the epiblast.

In addition to these we have the crystalline lens formed of involuted epiblast and the cavity of the mouth lined by it. These are the most important parts which are derived from the epiblast.

From the hypoblast are derived the epithelium of the digestive canal, the epithelium of the trachea, bronchial tubes and air cells, the cylindrical epithelium of the ducts of the liver, pancreas and other glands of the alimentary canal, as well as the hepatic cells constituting the parenchyma of the liver, developed as we have seen from the solid hypoblast cylinders given off around the primary hepatic diverticula.

Homologous probably with the hepatic cells, and equally of hypoblastic origin, are the spheroidal 'secreting cells' of pancreas and other glands. The epithelium of the salivary glands, though these so exactly resemble the pancreas, is of epiblastic origin, inasmuch as the cavity of the mouth (Chap. vi. § S) is entirely lined by epiblast.

The hypoblast also lines the allantois.

From the mesoblast are formed all the remaining parts of the body. 'The muscles, the bones, the connective tissue and the ressels, both arteries, veins, capillaries and lymphatics with their appropriate epithelium, are entirely formed from the mesoblast.

All the nerves of the body, both the cranial nerves (the so-called optic and olfactory nerves alone excepted), the spinal nerves, and the sympathetic system, are also formed from the mesoblast. The nerve-cells of the sympathetic ganglia as well as those of the ganglia on the posterior roots of the spinal nerves are of mesoblastic origin, and thus appar- 
ently are in striking contrast with the nerve-cells in the brain and cord. The fibres constituting the white matter of both brain and spinal cord are also probably derived from mesoblast.

The generative and urinary organs are entirely derived from the mesoblast. It is worthy of notice that their epithelium, though resembling so closely the hypoblastic epithelium of the alimentary canal, is distinctly mesoblastic.

From the mesoblast lastly are derived all the muscular, connective, and nervous and vascular elements, as well of the alimentary canal and its appendages as of the skin and the tegumentary organs. Just as it is only the epidermic moiety of the latter which is derived from the epiblast, so it is only the epithelium of the former which comes from the hypoblast.

In the present state of our knowledge we cannot in all cases with certainty say which parts of the mesoblast enter into the formation of particular organs; the more important facts in this part of our subject will however already have been gathered, from the earlier part of this work.

11. The important events then which characterize the fifth day are:-

1. The growth of the allantois.

2. The appearance of the knee and elbow, and of the cartilages which precede the bones of the digits and limbs.

3. The formation of the primitive cartilaginous cranium, more especially of the investing mass, the trabeculæ, and the ethmo-vomerine plate; and the appearance of rods of cartilage in the visceral arches.

4. The developments of the parts of the face: the closing in of the nasal passages by the nasal processes.

5 . The formation of the anus.

6. A large development of grey matter in the spinal cord as the anterior and posterior cornua ; considerable growth both of the anterior and posterior white columns, and the commencement of the anterior and posterior fissures.

7. The appearance of the auricular septum, of a septum in the bulbus arteriosus, and of the semilunar valves.

8. The establishment of the several tissues. 


\section{CHAPTER VIII.}

\section{FRON THE SIXTH DAY TO THE END OF INCUBATION.}

1. The sixth day marks a new epoch in the development of the chick, for distinctly avian characters then first make their appearance.

Striking and numerous as are the features, which render the class aves one of the most easily recognizable in the whole animal kingdom, the embryo of a bird does not materially differ in its early phases from that of a reptile or a mammal, even in the points of structure which are most distinctively avian. It may, it is true, be possible to infer, even at a comparatively early stage, from some subsidiary tokens, whether any given embryo belongs to this class or that (and indeed the same inference may be drawn from the ovum itself); but up to a certain late it is impossible to point out, in the embryo of the fowl, the presence of features which may be taken as broadly characteristic of an avian organization. This absence of any distinctive avian differentiation lasts in the chick roughly speaking till the commencement of the sixth day.

IVe do not mean that on the sixth day all the organs sudilenly commence to exhibit peculiarities which mark them as avian. 'There are no strongly marked breaks in the history of development; its course is perfectly gradual, and one stage passes continuously into the next. The sixth and seventh days do however mark the commencement of the period in which the specialization of the lird begins to be apparent. Then for the first time there become visible the main features of the characteristic manus and pes; the crop and the intestinal caca make their appearance; the stomach takes on the form of a gizzard; the nose begins 
to develope into a beak; and the commencing bones of the skull arrange themselves after an avian type. Into these details we do not propose to enter, and shall therefore treat the history of the remaining days with great brevity.

We will first speak of the FETAL APPENDAGES.

2. On the sixth and seventh days, these exhibit changes which are hardly less important than the events of previous days.

The amnion at its complete closure on the fourth day very closely invested the body of the chick; the true cavity of the amnion was then therefore very small. On the fifth day, fluid begins to collect in the cavity, and raises the membrane of the ammion to some distance from the embryo. The cavity becomes still larger by the sixth day, and on the seventh day is of very considerable dimensions, the fluid increasing with it. On the sixth day Von Baer observed movements of the embryo, chiefly of the limbs; he attributes them to the stimulation of the cold air on opening the egg. By the seventh day very obvious morements begin to appear in the amnion itself; slow vermicular contractions creep rythmically over it. The amnion in fact begins to pulsate slowly and rythmically, and by its pulsation the embryo is rocked to and fro in the egg. This pulsation is due probably to the contraction of involuntary muscular fibres, which seem to be present in the attenuated portion of the mesoblast, forming part of the amniotic fold. (Cf. Chap. II. \$ 9, p. 42.) Similar movements are also seen in the allantois at a considerably later period.

The growth of the allantois has been very rapid, and it forms a flattened bag, covering the right side of the embryo and rapidly spreading out in all directions, between the primitive folds of the amnion, that is between the amnion proper and the false amnion (or chorion). It is filled with fluid, so that in spite of its flattened form its opposite walls are distinctly separated from each other.

The vascular area has become still further extended than on the previous day, but with a corresponding loss in the definite character of its blood-ressels. The sinus terminalis has indeed by the end of the seventh day lost all its previous distinctness. And the vessels which brought back the blood from it to the heart are no longer to be seen. 
Both the omphalo-mesaraic arteries and veins now pass to and from the body of the chick as single trunks, assuming more and more the appearance of being merely branches of the mesenteric vessels.

The yolk is still more fluid than on the previous day, and its bulk has (according to Von Baer) increased. This can only be due to its absorbing the white of the egg, which indeed is diminishing rapidly.

3. During the eighth, ninth, and tenth days, the amnion does not undergo any very important changes. Its cavity is still filled with fluid, and on the eighth day its pulsations are at their beight, henceforward diminishing in intensity.

The splitting of the mesoblast has now extended to the outer limit of the vascular area, viz. over about three quarters of the yolk-sac. The somatopleure at this point is continuous (as can be easily seen by reference to Fig. 8) with the original outer fold of the amnion.

It thus comes about that the further splitting of the mesoblast merely enlarges the cavity in which the allantois lies. The growth of this organ keeps pace with that of the cavity in which it is placed. Spread ont over the greater part of the yolk-sac as a flattened bag filled with fluid, it now serves as the chief organ of respiration.

Hence it is very vascular, the vessels on that side of the bag which is turned to the chorion and shell being especially large and numerous.

The yolk now begins to diminish rapidly in bulk. The yolk-sac becomes flaccid, and on the eleventh day is thrown into a series of internal folds, abundantly supplied with blood-vessels. By this means the surface of absorption is largely increased, and the yolk is more and more rapidly taken up by the blood-vessels, and in a partially assimilated condition transferred to the body of the embryo.

4. By the eleventh day the abdominal parietes though still much looser and less firm than the walls of the chest may be said to be definitely established, and the loops of intestine, which have hitherto been hanging down into the somatic stalk, are henceforward confined within the cavity of the abdomen. The body of the embryo is therefore completed; but it still remains connected with its various 
appendages by a narrow somatic umbilicus, in which run the stalk of the allantois and the solid cord suspending the yolk-sac.

The cleavage of the mesoblast still progressing, the yolk is completely invested by the (splanchnopleuric) yolk-sac except at the pole opposite to the embryo, where for some little time a small portion remains unenclosed; at this spot the diminished white of the egg adheres as a dense viscid plug.

The allantois meanwhile spreads out rapidly, and lies over the embryo close under the shell, being separated from the shell membrane by nothing more than an attenuated membrane the chorion, formed out of the outer primitive fold of the amnion and the remains of the vitelline membrane. With this chorion the allantois partially coalesces, and in opening an egg at the later stages of incubation, unless care be taken the allantois is in danger of being torn in the removal of the shell membrane. As the allantois increases in size and importance, the umbilical (or allantoic) vessels are correspondingly developed. They are very conspicuous when the egg is opened, the pulsations of the umbilical arteries at once attracting attention.

5. On about the sixteenth day, the white having entirely disappeared, the cleavage of the mesoblast is carried right over the pole of the yolk opposite the embryo, and is thus completed (Fig. 8). The yolk-sac now, like the allantois which closely wraps it all round, lies loose in a space bounded outside the body by the chorion, and continuous with the pleuroperitoneal cavity of the body of the embryo. Deposits of urates now become abundant in the allantoic fluid.

The loose and flaccid walls of the abdomen enclose a space which the empty intestines are far from filling, and on the nineteenth day the yolk-sac, diminished greatly in bulk but still of some considerable size, is withdrawn through the somatic stalk into the abdominal cavity, which it largely distends. Outside the embryo there remains nothing now but the highly vascular allantois and the practically bloodless chorion and amnion. The amnion, whose fluid during the later days of incubation rapidly diminishes, is continuous at the umbilicus with the body-walls of the embryo. The 
chorion, (or outer primitive amniotic fold,) is by the completion of the cleavage of the mesoblast and the invagination of the yolk-sac, entirely separated from the embryo. The cavity of the allantois by means of its stalk passing through the umbilicus is of course continuous with the cloaca.

6. In the EMbrio itself a few general points deserve notice.

By the sixth or seventh day, the flexure of the body has 'become less marked, so that the head does not lie so near to the tail as on the previous days; at the same time a more distinct neck makes its appearance.

Though the head is still disproportionately large, its growth ceases to be greater than that of the body.

Up to this period the walls of the somatic stalk have remained thin and flaccid, almost membranous in fact, the heart appearing to hang loosely out of the body of the embryo. About this time however the stalk, especially in front, rapidly narrows and its mesoblast becomes thickened. In this way the heart and the other thoracic viscera are enclosed by definite firm chest walls, along the sides of which the ribs grow forwards and in front of which the cartilaginous rudiments of the sternum appear.

The abdominal walls are also being formed, but not to the same extent, and the stalk of the allantois still passes out from the peritoneal cavity between the somatic and the splanchnic stalks.

In the brain one of the most marked features is the growth of the cerebral hemispheres. The median division between these has in front increased in depth, so that the lateral ventricles are continued forwards as two divergent horns, while backwards they are also continued as similar divergent horns separated from one another by the vesicle of the third ventricle.

We propose to treat more fully of the development of the brain in a later part of this work, the importance of the mammalian brain rendering it unclesirable to go too much into the details of the brain of the bird.

All the visceral clefts, with the exception of the first, are closed by the seventh day: this one however still remains open, communicating with the mouth by the Eustachian tube and with the exterior by the aperture of the external auditory 
meatus. It becomes divided internally into two parts by the tympanic membrane.

The structures which surround the mouth are beginning to become avian in form, though the features are as yet not very distinctly marked. The inferior maxillary processes meet in front and form the lower boundary of the mouth; while, separated from these by only a narrow slit, the superior maxillary processes and fronto-nasal process meet in a similar way above, to form the upper boundary. The union of the superior maxillary processes is not with the tip of the frontonasal process, but with its sides, so that an angular space is left on each side between them (vide Fig. 58). The nasal grooves are however completely roofed over.

The tongue has appeared on the floor of the mouth as a bud of mesoblast covered by epiblast.

7. During the eighth, ninth, and tenth days, the embryo grows very rapidly, the head being still especially large, and at the same time becoming more round, the midbrain not being so prominent.

8 . From the eleventh day onwards the embryo successively puts on characters which are not only avian, but even distinctive of the genus, species and variety.

So early as the ninth or tenth day the sacs containing the feathers begin to protrude from the surface of the skin as papillæe especially prominent at first along the middle line of the back from the neck to the rump, and over the thighs, the sacs of the tail feathers being very conspicuous. On the thirteenth day, these sacs, generally distributed over the borly, and acquiring the length of a quarter of an inch or more, appear to the naked eye as feathers, the thin walls of the sacs allowing their contents, now coloured according to the variety of the bird, to shine through. They are still however closed sacs, and indeed remain such even on the nineteenth day when many of them are an inch in length.

On the eighth day a chalky-looking patch is observable on the tip of the nose. This by the twelfth day has become developed into a horny but still soft beak.

On the thirteenth day, nails are visible at the extremities, and scales on the remaining portions of the toes. These on the sixteenth day become harder and more horny, as does also the beak. 
By the thirteenth day the cartilaginous skeleton is completed and the various muscles of the body can be made out with tolerable clearness.

Ossification begins according to Von Baer on the eighth or ninth day by small deposits in the tibia, in the metacarpal bones of the hind-limb, and in the scapula. On the eleventh or twelfth day a multitude of points of ossification make their appearance in the limbs, in the scapular and pelvic arches, in the ribs, in the bodies of the cervical and dorsal vertebræ and in the bones of the head, the centres of ossification of the vertebral arches not being found till the thirteenth day.

Fig. 62.

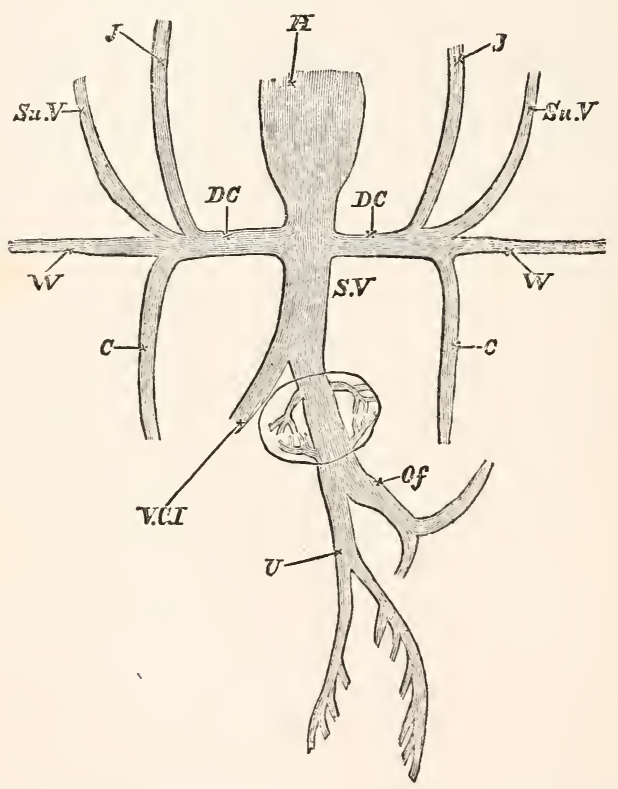

Diagram of the Venous Circulation at the Cominencement of the FiFth DaY.

II. heart. D.C. ductus Cuvieri. Into the ductus Cuvieri of each side fall $J$. the jugular vein, $S u$. $V$. the superior vertebral, $W$. the vein from the wing and $C$. the inferior cardinal vein. $S$. $V$. sinus venosus. Of. omphalo-mesaraic vein. $U$. umbilical vein, which at this stage gives off branches to the body-walls. V.C. I. inferior vena cava. 
9. The events which we have thus briefly narrated are accompanied by important changes in the arterial and venous systems.

The condition of the venous system at about the end of the third day was fully described in Chap. V. $\S 16$, and the changes which have taken place between that date and the latter lays of incubation may be seen by comparing the diagram Fig. $39 \mathrm{~B}$ with the diagrams Figs. 62 and 63.

On the third day, nearly the whole of the venous blood from the body of the embryo was carried back to the heart by two main venous trunks, the superior (Fig. $39 \mathrm{~B}, S u$. $V_{\text {.) }}$ and inferior (Fig. $39 \mathrm{~B}, C$ ) cardinal veins, joining on each side to form the short transverse ductus Cuvieri, both which in turn united with the sinus venosus close to the heart. As the head and neck continue to enlarge and the wings become developed, the single superior cardinal or jugular vein, as it is usually called (Fig. 62, J), of each side, is joined by two new veins: the vertebral vein ( $S u$. V.), bringing back blood from the head and neck, and the vein from the wing $(W)$.

The inferior cardinal or vertebral veins have their roots in the Wolffian bodies; they become developed, pari passu, with those organs, and may be called the veins of the Wolffian bodies. On the third day they are the only veins which bring the blood back from the hinder part of the body of the embryo.

About the fourth or fifth day, however, a new single venous trunk, the vena cava inferior (Fig. 62, V.C.I.), makes its appearance in the middle line, in a plane more dorsal than that of the cardinal veins. This, starting from the sinus venosus not far from the heart, is on the fifth day a short trunk running backward in the middle line below the aorta, and speeaily losing itself in the tissues above the Wolffian bodies. When the kidneys are formed it receives blood from them, and thenceforward enlarging rapidly becomes the channel by which the greater part of the blood from the hind limbs and the hinder part of the body finds its way to the heart. In proportion as this vena cava inferior increases in size, and the Wolffian bodies give place to the permanent kidneys, the posterior cardinal veins diminish. Communicating branches between them and it are established 
FIG. 63.

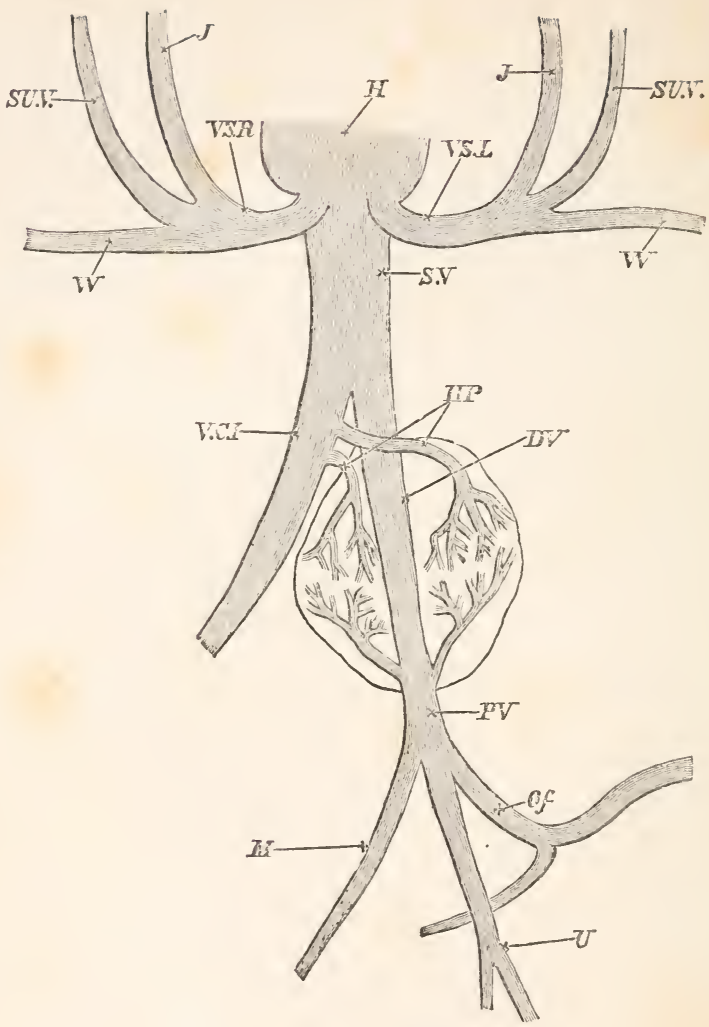

\section{Diagram of tife Vexous Circulation during the later Days of INCUBATION.}

II. heart. V.S.R. right vena cava superior. $\quad T$. S. $L$. left vena cava superior.

$S \cdot V$. sinus venosug. The two venæ cave superiores are the original 'ductus Cuvieri,' they still open into the sinus venosus and not independently into the heart. J.jugular vein. $S U . V$. superior vertebral vein. $W$. vein for the wing. $V$. C.I. vena cava inferior, which receives most of the blood from the inferior extremities, etc. IIP. Hepatic veins, which fall into the vena cava inferior. $D$. $V$. ductus venosus. $P . V$. portal vein. $M$. a vein bringing blood from the intestines into the portal vein. $O f$. omphalo-mesaraic vein. $U$. umbilical vein. The three last mentic ned veins unite together to form the portal vein.

The remnants of the inferior cardinal veins are not shewn. 
in the substance of the Wolffian bodies, so that soon the blood of those organs, as well as that from all the rest of the hind body, with the exception of the alimentary canal and its appendages, passes into the vena cava inferior.

The diminished trunks of the cardinal veins remain for some time; their anterior ends unite to form the small azygos vein.

At its first appearance the vena cava inferior may be considered as a branch of the trunk which we have called the sinus venosus, but as development proceeds, and the vena cava becomes larger and larger, the sinus venosus assumes more and more the appearance of being merely the cardiac termination of the vena cava, and the ductus venosus may now be said to join the vena cava instead of being prolonged into the sinus.

While this growth of the vena cava is going on, the points at which the ductus Cuvieri enter into the sinus venosus, or, as we may now say, vena cava inferior, are drawn in towards the heart itself, and finally these trunks fall directly and separately into the auricular cavities, and are henceforward known as the right and left vena cava superior (Fig. 63, V.S.R., V.S.L.). There are therefore, when these changes have been effected, three separate channels, with their respective orifices, by which the blood of the body is brought back to the heart, viz. the right and left superior and the inferior venæ cavæ.

While the auricular septum is as yet unformed, the blood from these veins falls into both auricles, perhaps more into the left than into the right. As the septum however grows up, the three vessels become connected with the right auricle only while the left receives the two pulmonary veins coming from the lungs. (Compare Chap. vir. § 7.)

On the third day the course of the vessels from the yolksac is very simple. The two omphalo-mesaraic veins, of which the right is already the smaller, form the meatus venosus from which, as it passes through the liver on its way to the heart, are given off the two sets of vence adrehentes and vence revehentes.

With the appearance of the allantois on the fourth day, a new feature is introduced. From the meatus venosus, a short distance behind the liver, there is given off a vein which quickly divides into two branches. These, running 
along the ventral side of the body from the walls of which they receive some amount of blood, pass to the allantois. They are the allantoic or umbilical veins. The single vein which they unite to form becomes, by reason of the rapid growth of the allantois, very long; and hence it is perhaps better to speak of it as the umbilical vein (Fig. 63, U). The right branch soon diminishes in size and finally disappears. Meanwhile the left on reaching the allantois bifurcates; and, its two branches becoming large and conspicuous, there still appear to be two main allantoic or umbilical veins uniting at a short distance from the allantois to form the single long umbilical vein. At its first appearance the umbilical vein seems to be but a small branch of the omphalo-mesaraic, but as the allantois grows rapidly, and the yolk-sac dwindles, this state of things is reversed, and the less conspicuous omphalo-mesaraic appears as a branch of the larger umbilical.

On the third day the blood returning from the walls of the intestine is insignificant in amount. As however the intestine becomes more and more developed, it acquires a distinct venous system, and the blood sent to it by branches of the aorta is returned by veins which form a trunk, the mesenteric vein (Fig. 63, $M$ ), falling into the omphalo-mesaraic vein at its junction with the umbilical vein.

These three great veins in fact, viz. the omphalo-mesaraic, the umbilical, and the mesenteric, form a large common trunk which enters at once into the liver, and which we may now call the portal vein (Fig. 63, P.V.). This, at its entrance into the liver, partly breaks up into the vence advehentes, and partly continues as the ductus venosus straight through the liver, emerging from which it joins the vena cava inferior. Before the establishment of the vena cava inferior, the venæ revehentes, carrying back the blood which circulates through the hepatic capillaries, joined the ductus venosus close to its exit from the liver. By the time however that the vena cava has become a large and important vessel it is found that the venæ revehentes or as we may now call them the hepatic veins have shifted their embouchment and now fall clirectly into that vein, the ductus venosus making a separate junction rather higher up (Fig. 63, $H P$ ).

This state of things continues with but slight changes till 


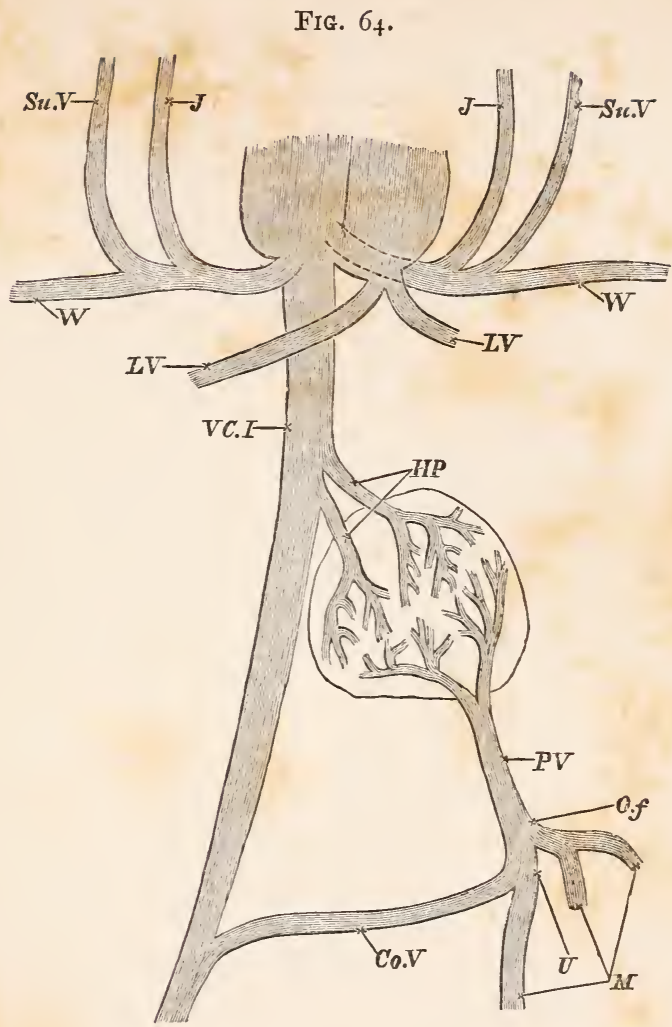

Diagray of the Venots Circulation of the Chick after the Comiencement of Respiration by means of the Lungs.

$W$. wing-vein. $J$.jugular vein. $S u$. $V$. superior vertebral vein. These unite together on each side to form the corresponding superior vena cava. $L$. $V$. pulmonary veins. $\quad V . C$. $I$. vena cava inferior. $H P$. hepatic veins. $P$. $V$. portal vein. $M$. mesenteric veins. At $U$. and $O . f$. are shewn the points at which the umbilical and omphalo-mesaraic veins originated previous to their becoming obliterated at the commencement of respiration. $C o$. $V$. connecting vessel between the branches of the portal vein and the vena cava inferior. It is called the coccygeo-mesenteric vein, and unites the cross branch connecting the two hypogastrics with the mesenteric vein. It is represented in the figure in a purely diagrammatic manner. The ductus venosus has become obliterated. The three venæ cavæ fall independently into the right auricle and the pulmonary veins into the left auricle. 
near the end of incubation, when the chick begins to breathe the air in the air-chamber of the shell, and respiration is no longer carried on by the allantois. Blood then ceases to flow along the umbilical vessels; they become obliterated. The omphalo-mesaraic vein, which as the yolk becomes gradually absorbed proportionately diminishes in size and importance, comes to appear as a mere branch of the portal vein. The ductus venosus becomes closed, remaining often as a mere ligament; and hence the whole of the blood coming through the portal vein flows into the substance of the liver, and so by the two hepatic veins into the vena cava (Fig. 64, HP).

Previous to these changes one of the veins passing from the rectum into the vena cava has given off a branch which effects a junction with one of the mesenteric veins. This now forms a somewhat conspicuous connecting branch between the systems of the vena cava and the portal vein (Fig. 64, Co.V.).

All three venæ cavæ now fall exclusively into the right auricle, and by the closure of the foramen ovale the blood

Frg. 65 .

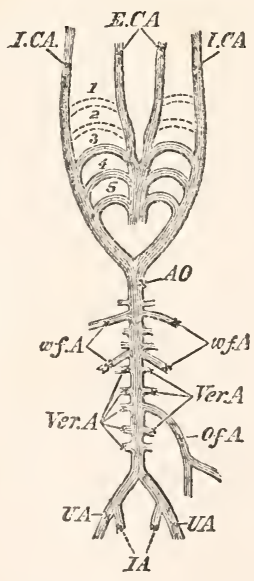

State of Arterial Circolation on the Fifth or Sixth Day. E. CA. extemal carotid. I. $C A$. internal carotid. AO. dorsal aorta. wf. $A$. arteries to the Wolffian bodies. Ver. A. arteries given off between each pair of vertebræ. Of. A. omphalo-mesaraic artery. UA. umbilical artery. IA. iliac artery. 
Alowing through them is entirely shut off from the left auricle, into which passes the blood from the two pulmonary veins (Fig. 6t, L.V.).

Such is the history of the veins in the chick. As will be seen in the second part of this work, the course of events in the mammal, though in the main similar, differs in some unimportant respects.

10. It remains for us to speak of the changes which have in the meantime been taking place in the arterial system. The condition of things which exists on the fifth or sixth day is shewn in the diagram (Fig. 65).

We have already seen (Chap. vI. p. 168) that of the three aortic arches which make their appearance on the third day, the first two disappear : the first on the fourth, the second on the fifth day; but that their disappearance is accompanied by the formation behind them of two new aortic arches, the fourth and the fifth. Thus there are generally three, never more than three, pairs of a.ortic arches present and functional at one time.

According however to Von Baer this is not strictly true. He states that there are four arches present both on the fourth and fifth days. In the case of the fourth day, a slight remnant of the first pair of arches still persists when the fourth pair is already formed; and on the fifth day the third pair has not entirely disappeared when the fifth pair is formed. In both of these cases kowever the first pair of arches of the four is only present for a very short time, and then is so diminished in size as to be of no importance.

The first pair of arches, before it entirely disappears, sends off on each side two branches towards the head. Of these, one forms the direct continuation of the bulbus arteriosus in a straight line from the point where the first aortic arch leaves it. Primarily distributed to the tongue and inferior maxillary region, it becomes the external carotid (Fig. 65, E. CA.).

The other, starting from the point where the aortic arch of each side joins its fellow above the alimentary canal to form the dorsal aorta, is primarily distributed to the brain, and becomes the internal caratid (Fig. 65, I.CA.).

When the first arch disappears, the external carotid arteries still remain as the anterior continuations of the bulbus arteriosus. And since the dorsal trunks uniting the listal ends of the first and second arches do not become obliterated at the time when the first pair of arches dis- 
appears, the internal carotids remain as branches springing from the distal ends of the second pair of arches; they are supplied with blood from that pair, the stream in which flows chiefly towards the head instead of backwards towards the dorsal aorta, as is the case with the succeeding arches. When the second pair of arches is obliterated, the connecting branch with the next arch is again left, and thus the internal carotids appear as branches' from the distal ends of the third pair of arches.

On the third day, the dorsal aorta does not long remain single in its backward course along the body, but soon divides into two trunks which run one on either side of the middle line of the body. On the fourth day however, the point at which the aorta divides is carried very much further back, quite to the posterior end of the Wolffian bodies. The two branches into which it there divides, form the origin of the iliac (Fig. 65, IA) arteries supplying the hind limbs. Each of these sends a short branch to the allantois $(U A)$. As the allantois grows rapilly and becomes an important respiratory organ, these allantoic or umbilical arteries increase so much in size that they speedily appear to be the direct continuations of the aorta, and the iliac arteries to be mere branches of them. As a general, though apparently not invariable rule, the right umbilical artery gets gradually smaller and soon disappears.

From the main trunk of the aorta are given off small transverse branches between the vertebræ (represented diagrammaticallyin Fig. 65, Ver. A.), and also important branches to the Wolffian bodies (Fig. 65, wf. A.).

The omphalo-mesaraic artery $(O f . A$.) now leaves the aorta as a single but quickly bifurcating trunk, which at the end of the fifth day is still very large.

By the fifth day, the ventricular portion of the heart (compare Chap. viI. $§ 7$ ) is almost completely divided into two chambers. The bulbus arteriosus is also divided by a septum into two channels, which do not however run in a straight course, but have, according to Von Baer, a spiral arrangement. One of the channels communicates with the right ventricle of the heart and the other with the left. The spiral of the former turns from the right and above (dorsal), to the left and below (ventral); the latter from the left and 
below (ventral), to the right and above (dorsal). The septum separating these two channels, according to Dr Tonge (Proc. Roy. Soc. 1868), commences as an outgrowth from the wall between the fourth and fifth pair of arches, and is so arranged that the channel from the left ventricle communicates with the third and fourth pairs of arches only, and that from the right ventricle with the fifth pair only.

According to Dr Tonge's view the two channels after the completion of the septum do not communicate with each other at any point, and are completely shut off from each other at their ends. Von Baer believed that the two channels (into which the bulbus arteriosus is divided by the septum) opened at their distal terminations into a common trunk, but that the direction of the openings of the two channels caused the two strearns of blood from them to enter into different arterial arches. According to him the stream from the channel communicating with the left rentricle is directed so as entirely to miss the last pair of arterial arches, and to fall into the third and fourth pairs, while that from the right ventricle enters the fifth pair alone.

One result of this arrangement is that all the blood which passes to the anterior extremity of the body, comes from the left ventricle of the heart.

At about the seventh day an entire separation begins to take place between the arterial roots which come respectively from the right and left chambers of the heart. The root from the right chamber (Fig. 66, R.P.A.) remains connected with the fifth pair of arches. The root from the left ventricle is connected with the third and fourth pairs of arches.

According to Von Baer the right arterial root is connected with the fourth arch on the left side, and the fifth arch on the right side. He also believed that the fifth arch on the left side has by this day altogether disappeared. According to his view, therefore, the pulmonary arteries are derived from both the fifth and fourth pairs of arches. Rathke (Denkschriften der Akademie $z u$ Wien, I857, Bd. xnII.) however altogether denies this, and states that both pulmonary arteries are derived from the fifth pair of arches alone. We have in our account followed Rathke's statements.

The lower part of the body still receives blood from both the right and left ventricles, since the blood which enters the fifth arch still flows into the common dorsal aorta.

As the lungs however increase in size, a communication is set up between them and the fifth pair of arches in the shape of two vessels which, springing one from the arch of each side, grow downwards towards the lungs. At first small and narrow, these pulmonary arteries, for such they are, grow rapidly larger and larger, so that more and more of the blood from the right ventricle is carried to the lungs. 
FIG. 66.

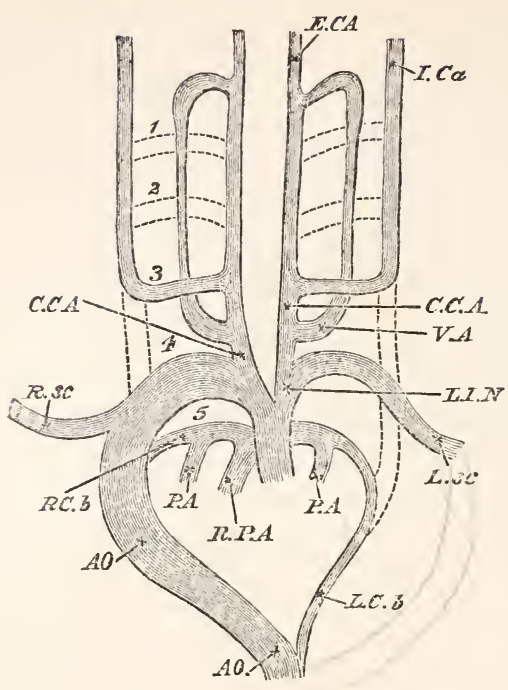

Diagray of the Condition of the Arches of the Aorta towards the Close of Incubation.

I , 2, 3, 4, 5. The several aortic arches. E.CA. External carotid. I.Ca. Internal carotid. C.C.A. Common carotid. V.A. 'Vertebral' artery. R.sc. Right subclavian. L.sc. Left subclavian. L.I.N. Left innominate. AO. Aorta. P.A. Pulmonary arteries. R.P.A. Right arterial root, or division of bulbus arteriosus, or pulmonary artery; the left root or division, constituting the aorta, is seen by its side. RC.b. Communication on the right side between the fourth and fifth arches. L.C.b. Communicating trunk between the fifth arch and the dorsal aorta.

At the same time the connection between the third and fourth pairs of arches on each side grows weaker; and finally the passage between them becomes obliterated, so that less and less of the blood which flows along the third pair of arches is able to pass backwards to the hind end of the body.

On the eighth day (according to Rathke, loc. cit.) from the common root of the external and internal carotids of each side, a branch (Fig. 66, V.A.) is given off which passes forwards along the neck, and ends in front by becoming connected with a branch from the external carotid. This vessel is of much larger calibre at its two extremities, than in the central part of its course. In the adult it terminates anteriorly by anastomosing with the occipital artery (a branch from the external carotid). The smaller calibre of the central part is still more marked in the adult, than at the stage we are 
describing. This vessel passes up in the neural arch of the vertebræ, and is usually spoken of as the vertebral artery.

Rathke calls the vertebral arteries the 'arteriæ collaterales colli,' and if his view of their development is correct they can scarcely be considered homologous with the vertebral arteries usually so called in mammals. Soon after the anastomosis between the third and fourth arches disappears, the common trunk of the carotids on each side becomes much lengthened, and it is from near the base of the lengthened common carotid that the 'vertebral artery' (as we shall call Rathke's 'arteria collateralis colli') takes its origin.

\section{The fourth arch of the right side now becomes the most} important of all the arches; and nearly the whole of the blood supplying the hinder parts of the body passes through it. It is this arch which remains as the permanent aortic arch of the adult; and it is important to notice that the arch which forms the great dorsal aorta in birds is the fourth on the right side, and not as in mammals the fourth on the left side.

From the observations of Rathke we know, with tolerable certainty, the manner in which the carotids and the so-called vertebral arteries of birds are developed. There is however still some doubt as to the origin of the subclavian arteries, although Von Baer and Rathke have both investigated the point.

Von Baer believed that the artery which forms the continuation of the third arch, which on Rathke's authority we have called the 'internal carotid,' became the 'vertebral artery;' and he believed that the subclavian was given off as a branch from it. As Rathke points out, this does not agree with the anatomy of the parts, since in birds the subclavian forms the continuation of the innominate artery, after a common branch for the vertebral and carotids has been given off trom it. If it had not been for Rathke's satisfactory observations on the development of the carotids and vertebrals, this would not be a fatal objection to Von Baer's view; since we might easily suppose that although the subclavian was originally a branch of the vertebral, yet by subsequent changes, the point at which the subclavian left the vertebral was carried further and further back, till finally the subclavian became a branch of the common trunk of the vertebral and carotids, or in other words the subclavian formed the continuation of the innominate artery, after the common branch which divides into the vertebral and carotids had been given off from it.

Rathke's view of the origin of the subclavian is founded on the analogy of other vertebrates, rather than on his own observations on the chick. He states that although he attempted to do so, he was unable satisfactorily to observe the origin of these vessels in the chick. The following is the view which he adopts, and which we have followed in our diagram (Fig. 66).

The right subclavian (R.sc.) arises, he believes, either from the connecting branch (anastomosis) between the fourth and fifth arches (of that side), or from the branch connecting the fifth arch with the dorsal aorta; probably from the former. This would make its development very nearly similar to the development of the corresponding subclavian (i.e. the left) in mammals. We shall mention directly, in speaking of the final changes which the arterial system undergoes, how it is that the right subclavian fnally comes to form the continuation of the right innominate artery.

The left subclavian ( $L$. sc.) forms the continuation of the fourth arch 
of the left side, and primarily takes its origin from the connecting branch between the fourth and fifth arches. Its mode of development thus nearly agrees with that of the right subclavian of mammals. In support of this view of the development of the left subclavian, is the fact that in some birds there is, between it and the dorsal aorta, a fibrous connection which is the remains of the vessel which originally carried the blood from this fourth arch to the dorsal aorta. On the left side therefore, as can easily be understood by reference to the diagram, the subclavian without further alteration remains as the continuation of the left innominate, L.I.N.

In consequence of these changes the condition of the aortic arches during the latter days of incubation, before respiration by the lungs has commenced, is as follows (Fig. 66).

The first and second arches are completely obliterated. The third arch on each side is continued at its distal end as the internal carotid, I.Ca, the connection between it and the fourth arch having become entirely obliterated. From its proximal end as the direct continuation of the trunk which originally supplied the first and second arches the external carotid, E.CA., is given off. Each pair of carotids arises therefore from a common trunk-the common carotid $(C$. C.A.). Each of these trunks gives off near its proximal end a branch, the vertebral artery (V.A.), which joins at its distal end a branch from the external carotid.

The common carotid on the right side comes off from the fourth arch of the right side (the arch of the dorsal aorta), and is not as yet connected with the right subclavian, R.sc.

The common carotid of the left side comes off from the fourth arch of the left side; but since this arch becomes the left subclavian, L.sc. (the connection between the fourth and fifth left arches being obliterated), the portion of the trunk (L.I.N.) between the fourth arch and the bulbus arteriosus (or as it must now be called the common aortic root) is called the left innominate artery.

The fourth arch of the right side forms the commencement of the great dorsal aorta, and gives off the right subclavian ( $R . s c$. ) just before it is joined by the fifth arch.

The fifth arch of each side gives off branches $P$.A. to the lungs; their distal continuations RC.b., L.C.b., by which they are connected with the systemic circulation, though much reduced, are not obliterated.

The final changes undergone by the arterial system after the commencement of the pulmonary respiration consist 
chiefly in the complete separation of the pulmonary and systemic circulations.

FIG. 67.

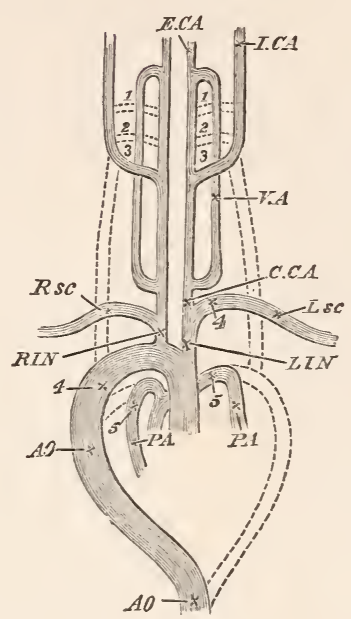

Diagray of the Arterial Ststem of the Adult Fowl.

R.I.N. right innominate artery. The other letters as in Fig. 66.

The dotted lines, as before, shew the portions of arches which have been obliterated.

As the branches to the lungs become stronger and stronger, less and less blood from the right ventricle enters into the dorsal aorta; and the connecting vessels become smaller and smaller.

Each of the arches from the right ventricle may therefore be considered at about the sixteenth or eighteenth day as divided into two parts, an inner part which connects the heart with the lung, and an outer part which still connects the arch with the main dorsal aorta. As these outer parts become smaller they receive the name of the 'ductus or canales Botalli' or 'ductus arteriosi.' The one on the right side is short; that on the left side is much longer and narrower.

Von Baer supposed that the reason of this was, that since the pulmonary arch of the left side was the fourth, the ductus Botalli of that side consisted of the branch between the fourth and fifth arch, as well as between the fifth arch and the dorsal aorta. It is easy however from diagram (Fig. 66) to see that this 
reason is superfluous, and that the explanation is that the canalis Botalli of the right side is merely the portion of the fifth arch between the origin of the pulmonary artery and the junction of the fifth arch with that common trunk, with which all the arches of that side are at one time or other connected, and which remains as the continuation of the aortic arch into the dorsal aorta; while on the left side it consists of the same portion of the arch as on the right side, and also of the corresponding common trunk of the left side.

When respiration commences the blood ceases to pass through these canals, which either remain as mere ligaments or else become absorbed altogether. By this means, the foramen ovale becoming at the same time closed, a complete double circulation is established. All the blood from the right ventricle passes into the lungs, and all that from the left ventricle into the body at large.

Two other changes take place about the same time in the aortic branches. That portion of the right fourth or aortic arch which lies between the origin of the right subclavian and the common carotid becomes shortened, and is finally swallowed up in such a fashion that the right subclavian (Fig. 67, R.sc.) comes off from the right common carotid, a very short trunk being left between the union of the two to serve as the right innominate artery.

At the same time, corresponding to the increase in the length of the neck, the common carotids are very greatly lengthened. They lie close together in the neck, and in many birds actually unite to form a common trunk.

It will of course be understood that with the disappearance of the allantois and the absorption of the yolk, the umbilical and omphalo-mesaraic arteries also disappear.

11. It may perhaps be of advantage to the reader if we here briefly summarize the condition of the circulation at its four most important epochs; viz. on the third day, on the fifth day, during the later days of incubation before respiration by the lungs has commenced, and after the chick has begun to breathe by the lungs.

On the third day the circulation is of an exceedingly simple character.

The heart is to all intents and purposes a simple twisted tube marked off by constrictions into a series of three consecutive chambers. The blood coming from the venous radicles passes through the heart and then through the three pair of arterial arches. 
From these it is collected into the great dorsal aorta. On this dividing into two branches, the stream of blood also divides and passes down on each side of the notochord along the body, and thence out by the omphalo-mesaraic arteries, which distribute it to the yolk-sac.

In the yolk-sac it partly passes into the sinus terminalis and so into the fore and aft trunks, partly directly into the lateral trunks, of the omphalo-mesaraic veins. In both cases it is brought back to the two venous radicles and so to the heart.

On this day the blood is aerated in the capillaries of the yolk-sac.

On the fifth or sixth day, the two auricles are present though having a common cavity. The septum of the ventricles is nearly complete, so that the blood on entering the ventricles from the auricles is divided into two streams. These two streams pass respectively from the right and left chambers of the heart into the two divisions of the bulbus arteriosus. The blood from the right ventricle passes into the fifth pair of arches and that from the left ventricle into the third and fourth pairs of arches.

From the anterior parts the blood is brought back by the anterior cardinal or jugular veins; from the hinder parts of the body, chiefly by the cardinal veins, but also in part by the now commencing vena cava inferior.

The blood from the yolk-sac and allantois, together with a small quantity from the intestine, is collected into the portal vein, and by that vessel carried to the liver. Here it becomes divided into two streams, part flowing directly by the ductus venosus into the sinus venosus, and the remainder passing through the capillaries of the liver and being brought back to the sinus venosus by the hepatic veins.

During this period the blood is aerated both by the allantois and yolk-sac, but as yet chiefly by the latter.

At a somewhat late period of incubation, the blood from the ventricles passes into two entirely distinct roots. The one of these, that from the right chamber, sends the blood to the fifth pair of arches. Passing through these two arches, the greater part of the blood flows into the dorsal aorta, a small portion only finding its way into the lungs through the as yet unimportant pulmonary arteries. 
Through the other aortic root, viz. that from the left ventricle, the blood flows into the third and fourth pairs of arches. That part of the blood which flows into the third pair of arches, passes almost entirely to the head and upper extremities by the external and internal carotids; that which flows into the right arch of the fourth pair, is chiefly brought to the dorsal aorta, but some of it passes to the right wing; that, on the contrary, which goes into the left fourth arch, is for the most part sent to the wings, a small part only reaching the dorsal aorta. There is still a mixture of the blood from the two chambers of the heart, so that the blood in the dorsal aorta is composed partly of the blood from the left, and partly from the right chambers. The blood of the upper (anterior) end of the body comes entirely from the left ventricle.

The blood of the dorsal aorta passes to the yolk-sac and allantois, and to all the hinder parts of the body. It is brought back from the yolk-sac, from the allantois, and to a certain extent from the intestines, by the portal vein, part of the blood from which passes to the inferior vena cava by the direct course (ductus venosus), and part indirectly by the more circuitous course of the capillaries of the liver and hepatic veins.

The blood from the generative and urinary organs, and from the hinder extremities, is brought back to the heart by the vena cava inferior; that from the upper extremities and head by the jugular, vertebral and wing veins into the two venæ cavæ of the right and left side, and so to the heart. Of these three venæ cavæ, the left superior and the inferior open into the heart independently. The right superior enters with the inferior. All of these enter the right auricle, but the common opening of the inferior and right superior venæ cavæ is so directed, that the blood carried by those vessels flows chiefly through the foramen ovale into the left auricle. The blood from the left superior vena cava enters the right auricle only. Now the blood of the inferior vena cava has been partly aerated by the allantois; and, since it is this blood which passing through the left auricle and ventricle is distributed to the third aortic arch, unmixed by any blood from the right ventricle (the mixture with the blood from the fifth arch taking place in the fourth arch only), it happens that the blood which flows to the anterior extremities and 
head, is more aerated than that in any other part of the body.

From the anterior extremities the blood is to a great extent returned by the left superior cava, and goes into the right auricle, whence, by the right ventricle, it is distributed through the fifth pair of arches over the body, after joining the more aerated blood passing through the fourth pair of arches.

The blood from the lungs is brought back by two small veins into the left auricle.

The characteristics of the circulation at this time, are that the blood is aerated by the allantois, and that there is a partial double circulation. (Vide Chap. vir. § 9.)

As soon as respiration commences, the canals leading to the dorsal aorta trom the fifth pair of arches, which communicate only with the right ventricle, become closed. The blood passing along the fifth arch now flows only into the lungs, through the pulmonary arteries. The blood from the left ventricle owing to the cessation of the circulation of the yolk-sac and the allantois is distributed exclusively to the body of the chick, from whence it is all brought back into the right auricle by the three now independently opening venæ cavæ.

The portal veins henceforward receive blood from the intestines only, and the ductus venosus is obliterated, so that all the blood of the portal vein passes through the capillaries of the liver.

The partition between the auricles is rendered complete by the closure of the foramen ovale; into the right auricle the veins of the body enter, and into the left the pulmonary veins.

There is thus a completely double circulation formed, in which all the blood of the left ventricle is arterial, and all the blood of the right ventricle venous, and there is at no part of the circulation a mixture of venous and arterial blood.

12. As early as the sixth day, movements, as we have said, may be seen in the limbs of the embryo upon opening the egg. We may conclude that after this epoch spontaneous movements occur from time to time in the unopened egg. They cannot however be of any great extent until the fourteenth day, for up to this time the embryo retains the position 
in which it was first formed, viz. with its body at right angles to the long axis of the egg.

On the fourteenth day a definite change of position takes place; the chick moves so as to lie lengthways in the egg, with its beak touching the chorion and shell membrane where they form the inner wall of the rapidly increasing airchamber at the broad end (Chap. I. \$2).

On the twentieth day or thereabouts, the beak is thrust throngh these membranes, and the bird begins to breathe the air contained in the chamber. Thereupon the pulmonary circulation becomes functionally active, and at the same time blood ceases to flow through the umbilical arteries. The allantois shrivels up, the umbilicus becomes completely closed, and the chick piercing the shell at the broad end of the egg with repeated blows of its beak, casts off the dried remains of allantois, amnion and chorion, and steps out into the world. 


\section{CHAPTER IX.}

\section{THE DEVELOPMENT OF THE SKULL.}

1. Is the chapter on the fifth day, we gave a short description of the earliest stages of the development of the skull. The subject is however of sufficient importance to merit a separate chapter, and in order to render the present account complete in itself, we have found it necessary to repeat a few of the statements already made.

2. In its earliest condition the cranium is composed of a mesoblastic tissue of stellate cells which can be distinguished from the remainder of the mesoblast by its greater opacity. In this condition (which is that of the fourth day), it may be spoken of as the membranous cranium. From this membranous condition the tissue composing it rapidly passes into true hyaline cartilage.

3. The primitive skull ${ }^{1}$, from its very first formation on the fourth day, consists of elements which fall into two very distinct divisions. We have on the one hand a sheet of cartilage which ensheaths the notochord from its anterior end to the first vertebra. This sheet of cartilage forms an unsegmented continuation of the vertebral bodies. It is to be considered as the most anterior portion of the axial skeleton, in which the segmentation has become obliterated; and as such is equivalent not to one, but to a (hitherto not certainly determined) number of vertebræ.

This sheet was spoken of by Rathke (Entwickelungsgeschichte der Natter), its discoverer, as the 'investing mass,'

1 The facts narrated in this chapter are mainly derived from MIr Parker's Memoir upon the Development of the Skull of the Common Fowl (Gallus domesticus), Phil. Trans., r866, Vol. cLvi., pt. x. 
from its relations to the notochord, and as such we shall continue to speak of it.

The second of the two divisions into which the parts of the skull fall, consists of a series of paired rods, whose proximal extremities are attached more or less closely to the investing mass. All of these (with the exception of the trabeculæ) are developed along the axes of the visceral arches.

Fig. 68.

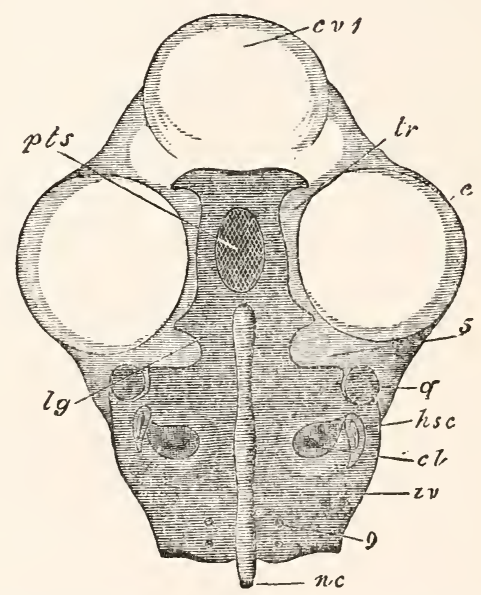

View From above of the Investing Mass and of the Trabecule on the Fourth Day of Incubation. (From Parker.)

In order to shew this the whole of the upper portion of the head has been sliced away. The cartilaginous portions of the skull are marked with the dark horizontal shading.

$c v$ I cerebral vesicles (sliced off). $e$ eye. $n c$ notochord. $i v$ investing mass. 9 foramen for the exit of the ninth nerve. cl cochlea. hsc horizontal semicircular canal. $q$ quadrate. 5 notch for the passage of the fifth nerve. $l g$ expanded anterior end of the investing mass. pts pituitary space. $t r$ trabeculæ. The reference line $t r$ has accidentally been made to end a little short of the cartilage.

We shall commence by describing the 'investing mass' as we find it on the fourth day, and from this pass on to the paired rods attached to it. 
4. The investing mass (Fig. 68 , iv) is on the fourth day a broad plate of tissue ensheathing the notochord and arching upwards on each side and especially behind. Laterally it encloses the auditory sacs, and the tissue surrounding these (forming the so-called 'periotic capsules') is in the chick never separate from the investing mass. In front it becomes narrowed, and at the same time excavated so as to form a notch on each side (Fig. 68, 5) through which the fifth nerve passes; and in front of this again it becomes expanded.

In order to render our subsequent account more intelligible, we may shortly anticipate the fate of the investing mass. Behind it grows upwards, and the two outgrowths meet above so as completely to enclose the medulla oblongata, and to circumscribe a hole known as the 'occipital foramen.' And it is at this point only that the roof of the skull is at any period formed of cartilage.

Later than this there appear in the investing mass a series of ossifications forming the whole of the occipital bone, and the skeleton of the ear. Knowing this, we shall be able to speak of the different parts of the investing mass, as the regions of the various bones 'ex-occipital,' 'basi-occipital' etc. even before the ossifications, which mark these out, have appeared.

5. In considering the paired rods of cartilage attached to the investing mass, we will commence with the most anterior. These are known as the 'trabeculce cranii' (Fig. 68, tr). They are two narrow rods whose proximal ends are attached to the front end of the investing mass, of which they appear like a forward continuation, while distally they unite with each other. There is thus left an oval (or more nearly circular) space where the cartilage forming the base of the skull is deficient. This space is enclosed behind by the investing mass, and on the two sides and in front by the trabeculæ; it is called the 'pituitary space' (Fig. 68, pts), and in it lies the 'pituitary body.'

Where the trabeculæ unite in front, they form a somewhat expanded plate of cartilage continued anteriorly into two diverging horns, which subsequently develope into the ali-nasal cartilages. Owing to the cranial flexure the trabeculæ at first lie in a different plane from the investing mass.

In function, the trabeculæ seem almost to serve as a con- 
tinuation of the base of the skull, and such they were considered to be by Rathke, their discoverer. Their different mode of development, and (in the lower vertebrates) primitive independence of the investing mass, prove that this is not the case, and that they are almost undoubtedly to be looked on as paired appendages. Their primitive independence of the investing mass is clearly shewn in the skulls of the Marsipobranchii, in which the trabeculæ are formed of a dense fibrous tissue and the investing mass of hyaline cartilage. (W. Mïller, Bau der Hypophysis u. des Processus infundibuli cerebri. Jenaische Zeitschrift, Vol. vi.) A very probable view, first put forward by Huxley in his Hunterian lectures (vide Anat. Vertebrates, p. 77), and subsequently adopted by Parker as well as other investigators, is, that they are homologous with the rods of cartilage developed in the visceral arches, and that they are therefore the remnants of an anterior pair of arches.

Gegenbaur (Vergleichende Anatomie der Wirbelthiere, IIr. Heft) looks upon the trabecule and their coalesced extremities simply as a prevertebral portion of the cranium, and not as paired appendages of the investing mass. Their paired condition seems to militate against this view; it must be admitted however that our present knowledge of them does not permit us to state with certainty more than that they are not morphologically a continuation of the investing mass.

In the bird and apparently many higher vertebrates they are never independent of the investing mass, and it was this fact which led to the erroneous view of their nature, formerly adopted, that they were forward continuations of the axial skeleton.

In them and in the plate of cartilage formed by their coalescence in front appear the ossifications of the whole of the sphenoid, the ethmoid and nasal regions.

6. The remainder of the paired series of appendages are developed in the visceral arches. The foremost of these are the cartilaginous rods developed in the first visceral or mandibular arch. In our account of the face we mention that the mandibular arch on each side produces a bud known as the superior maxillary process, which goes to form the superior boundary of the mouth. In this process, as well as in the primitive arch, rods of cartilage appear.

The segmentation, so to speak, of the first visceral arch 
appears when taken by itself somewhat remarkable, and led to the view, formerly very general and still held by many, that the superior maxillary process was equivalent to a visceral arch. Mr Parker has recently shewn that in both Osseous fishes and the Elasmobranchii all the anterior arches undergo a somewhat similar segmentation, and it appears therefore to be a fair deduction that this segmentation persists in the chick in the mandibular arch, while it has been lost in the others. None of Mr Parker's investigations on the lower vertebrates support the view that the maxillary process is an aborted anterior arch. Similar views as to the nature of the maxillary process have been arrived at by Gegenbaur (loc. cit.), from his investigations upon the crania of the cartilaginous Fishes.

7. The cartilage or differentiated mesoblast appears in the maxillary process later than in the mandibular arch,

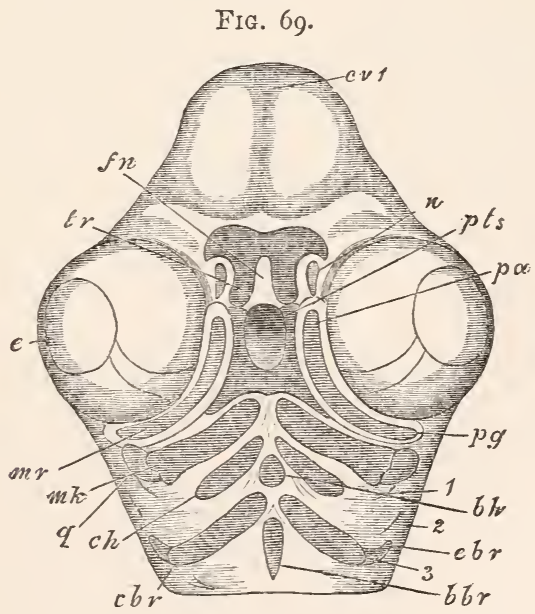

View from below of the Paired A ppendages of the Skold of a Fotrl on the Fourth Day of Incubation. (From Parker.)

$c v$ I cerebral vesicles. $e$ eye. fin fronto-nasal process. $n$ nasal pit. $t r$ trabecula. pts pituitary space. $m r$ superior maxillary process. $p g$ pterygoid. pa palatine. $q$ quadrate. $m k$ Meckel's cartilage. $c h$ cerato-hyal. $b h$ basi-hyal. $c b r$ ceratobranchial. $e b r$ proximal portion of the cartilage in the third visceral arch. $b b r$. basibranchial. I first visceral cleft. 2 second visceral cleft. 3 third visceral arch. 
and consists of a rod on each side. Each of these early becomes divided into two parts, a proximal and a distal. From the bones which subsequently develope in them the proximal one is known as the pterygoid (Fig. 69, pg), and the distal one as the palatine (Fig. $69, p a$ ). Both of the rods are very delicate, and remain in the cartilaginous condition for a short time only.

8. In the mandibular arch itself, there is also a proximal and distal cartilage. The proximal cartilage is situated (Figs. 68 and $69, q$ ) at the side of the investing mass but not united with it. It is known as the quadrate, and in the early stage is merely a small knob of cartilage. The distal rod is called Meckel's cartilage (Fig. $69, \mathrm{mk}$ ); on it are subsequently moulded the bones which form the mandible; while its proximal end becomes the articulare.

9. In the next arch, usually called the second viscera! or hyoid arch, there is a very small development of cartilage. This consists of a central azygos piece the 'basi-hyal' (Fig. 69, $b h$ ), and two rods, one on each side, the 'cerato-hyals' (Fig. 69, ch).

10. In the third arch, which corresponds with the first branchial arch of the Ichthyopsida, there is on each side a large distal cartilaginous rod (Fig. $69, c b r$ ) the 'ceratobranchial,' and a smaller proximal piece (Fig. 69, ebr); between the two arches lies an undefined mass (Fig. 69, bbr) the 'basibranchial.' In the arches behind this one there is in the bird no development of cartilage.

11. The growth of this primordial cartilaginous skull is very rapid, and by the 5 th or 6 th day very important changes have taken place. In the first place the pre-pituitary portion of the cranio-facial axis becomes equal to and subsequently actually surpasses in length the post-pituitary part. This change is accompanied by a considerable decrease in the cranial flexure. In the investing mass, the chief changes are an upward growth on each side behind, to form the exoccipitals; and the appearance of the occipital condyles (Fig. 70 , oc) as small swellings on each side of the middle line at the hind end of the plate. In front of the termination of the notochord two transverse vertical walls of cartilage rise up, the one in front of and the other behind the pituitary body: these are called respectively the anterior and 
posterior clinoid walls. But it is in front of the foremost of these that the most noticeable changes take place. From the mid-line of the coalesced trabeculæ there rises up a high ridge, the ethmo-presphenoid plate. This plate is at first highest behind. In front and below it sends out a process, the prenasal cartilage, which forms the cartilaginous basis on which the premaxillary region is moulded.

12. Development continues to be very rapid in these parts; and on the seventh day the anterior end of the ethmo-presphenoid plate (Fig. 70 , eth and $p s$ ) becomes its highest point and forms the retral spike of the ethmoid (Fig. 70, eth).

FIG. ;O.

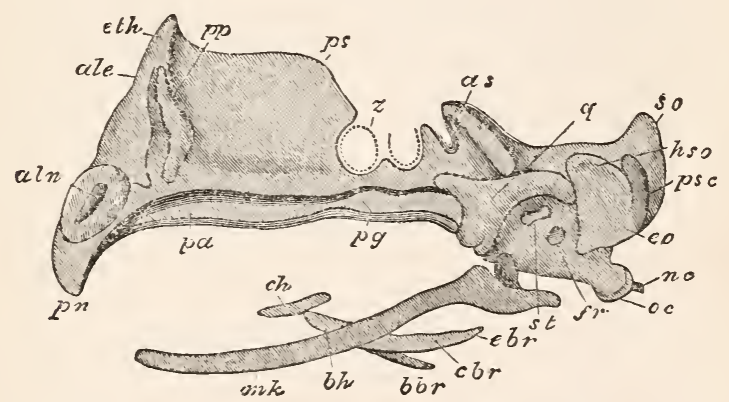

Side View of the Cartilaginous Cranium of a Fowl on the Seventh Day of Incubation. (From Parker.)

$p n$ prenasal cartilage. aln alinasal cartilage. ale aliethmoid ; immediately below this is the aliseptal cartilage. eth ethmoid. $p p$ pars plana. $p s$ presphenoid. $p a$ palatine. $p g$ pterygoid. $z$ optic nerve. as alisphenoid. $q$ quadrate. $s t$ stapes. fr fenestra rotundum. hso horizontal semicircular canal. psc posterior vertical semicircular canal : both the anterior and the posterior semicircular canals are seen shining through the cartilage. so supraoccipital. $e o$ exoccipital. $o c$ occipital condyle. $n c$ notochord. $m k$ Mleckel's cartilage. $c h$ cerato-hyal. $\quad b h$ basi-hyal. $c b r$ and $e b r$ ceratobranchial. $b b r$ basibranchial.

The prenasal cartilage (Fig. 70, pn) still points downwards, and by this time are formed the alinasal cartilages (Fig. 70, aln) developed from the trabecular horns, and the aliseptal cartilages which enclose the inferior turbinals (Fig. 70).

The basisphenoid grows outwards on each side to form 
the alisphenoid (Fig. 70,as) and posteriorly the supraoccipitals (Fig. 70, so) have approached much nearer to each other above; and at the same time the thickenings to form the occipital condyles have greatly increased (Fig. 70, oc).

The quadrate (Fig. 70, q) has undergone great modifications. In the earlier stage it was a simple knob of cartilage, but now it sends a process forwards, the orbital process, and a long process backwards which articulates with both the 'periotic capsule' and the exoccipital (Fig. 70, eo); downwards it sends a two-headed process to articulate with the extremity of Meckel's cartilage.

The fenestra ovale and fenestra rotundum (Fig. 70, fri) appear during this stage, and in the former the head of the small stapes (Fig. 70, st) is placed.

The palatine (Fig. 70, pa) and pterygoid (Fig. 70, pg) bars have increased in length, the former being the longer; between them and the base of the skull there has appeared a mass of tissue which will eventually become the sphenoidal rostrum (parasphenoid).

The second arch is not changed much; while the parts of the third arch, corresponding with the first branchial arch of osseous fishes, have increased in size, but have not undergone other modifications.

13. In the next (Parker's third) stage, which occurs about the middle of the 2nd week of incubation, the prenasal cartilage (Fig. 71, pn) has greatly increased in length and lost its downward curvature, so that it is in the same straight line as the septum nasi and the ethmoid; the continuous plate thus formed while increasing in length is at the same time narrowed except just in front of the pituitary space, where it becomes expanded on each side into an ear-shaped process.

The pituitary space is still open and admits the internal carotids; behind it is another azygos slit (Fig. 71, nc') along the median line, in which the notochord can be seen. This slit is a new formation, and as the skull outgrows the notochord, the surrounding parts will become the post-pituitary part of the basisphenoid.

The outgrowths forming the alisphenoids have largely increased. When looked at from below they are almost entirely concealed by the ossified basitemporals (Fig. 71, bt), 
but their anterior corners just project out in front of it (Fig. 71, as).

The occipital condyle formed by the coalescence of the two originally separate cartilaginous knobs, is seen just below the notochord (Fig. 71,nc), and the supraoccipitals (Fig. 71, so) have coalesced above the occipital foramen.

14. The ossifications of the cartilaginous skull, which have commenced at this stage, are ${ }^{1}$ :

(1) An ectosteal ossification around the notochord on the inner surface of the skull immediately behind the occipital condyle. This soon spreads to the opposite surface and forms an ossifying centre for the basioccipital.

(2) An ectosteal ossification (Fig. 71,eo) in each exoccipital beginning immediately behind the vagus foramen (Fig. 71, 8); this ossification commences on the exterior, but soon spreads round to the inner surface of the cartilaginous exoccipital.

(3) The palatine (Fig. 71, pa) has become entirely ossified by endostosis, the only instance of this process in the ossification of the primordial skull of the bird.

(4) An ectostosis of the pterygoid (Fig. 71, pg).

(5) An ectosteal ossification of the quadrate (Fig. 71, q).

(6) An ectosteal ossification of the cerato-branchials.

15. At this stage the majority of the true membrane bones also begin to be formed.

The paired premaxillaries (Fig. $71, p x$ ) are formed in the investment of the prenasal cartilage; they are triangular with the apex directed forwards, and soon begin to develope their three normal processes: one above towards the frontal (the nasal process), one along the margin of the beak to join the maxillary (the marginal process), and one below along the middle of the palate, to join the palatine bones. The maxillaries (Fig. $71, m x$ ) are developed on each side outside the endoskeleton. Pointed at each end, they are broader in the middle, sending a process inwards

1 The term ectostosis or ectosteal is used when ossification sets in between the perichondrium and the cartilage, endostosis when ossification takes place between the actual cells of the cartilage. These two processes not unfrequently occur combined, when ossification by ectostosis on reaching the cartilage sets up a true endosteal ossification. Parostosis is used for all ossifications which take place in purely fibrous tracts. Parosteal products are often spoken of as membrane or splint bones. 


\section{FIG. 7 I.}

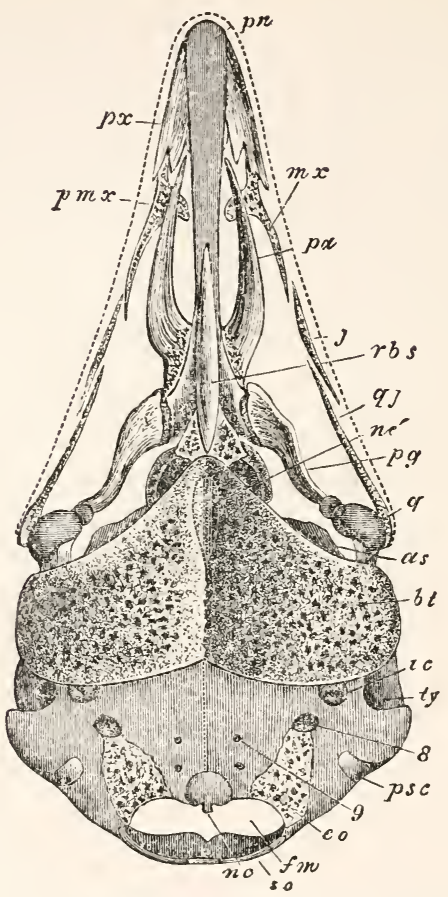

Eurbryonic Skull of a Fowl during the Second Week of Incubation (Third Stage) Frov Below. (From Parker.)

The figure is intended to shew the cartilaginous skull with its ossifications, and also the splint bones which have commenced to be formed independently of the cartilaginous cranium. The parts of the lower jaw and hyoid are omitted.

The following letters refer to the cartilaginous cranium and its ossifications. 2m prenasal cartilage. pa palatine bone (which has already become ossified). $p g$ pterygoid (also ossified). $n c^{\prime}$ notochord visible in the post-pituitary space. Both the notochord and the post-pituitary space would be in reality perfectly concealed by the plate of bone $b t$, but have been diagrammatically represented as if they were visible through it. $n c$ end of notochord appearing beyond the occipital condyle. $q$ quadrate. as alisphenoid. ic internal carotid. ty tympanic cavity. $p s c$ posterior semicircular canal. 8 foramen for passage of eighth nerve. 9 foramen for passage of ninth nerve. $e$ c centre of ossification for the occipital. so supraoccipital region. $f m$ foramen magnum. The following letters refer to the splint bones.

$p x$ premaxillary. $m x$ maxillary. $p m x$ process inwards from the premaxillary to form the maxillo-palatine. $j$ jugal. $q j$ quadrato-jugal. $r b s$ sphenoidal rostrum. $b t$ basitemporal. 
to form the maxillo-palatine (Fig. 71, pmx). The jugal (Fig. $71, j$ ) and the quadrato-jugals (Fig. 71, qj) are also developed during this stage, and connect the hinder end of the maxillaries with the quadrate; they are both delicate rod-like bones, the quadrato-jugal being the larger.

The top of the cranium, which up to this time has not been covered by either cartilage or bone, begins now to be closed in by parosteal osseous deposits taking place to form the nasals, frontals, and parietals. Outside the prootic region the squamosal also begins to be formed by a deposit taking place in the mesoblast external to the perichondrium.

In the base of the skull, there are three osseous deposits to form membrane bones, which soon uniting constitute the equivalent of the parasphenoid of osseous fishes.

The first of these is the osseous deposit which takes place in the mass of tissue, previously spoken of as the rostrum (Fig. 71, rbs); this is connected behind with two osseous deposits, the basisphenoidal ossicles, formed one on each side in front of the pituitary space. The third deposit is the large somewhat reniform mass of bone (Fig. 71, bt) called the basitemporal, which forms one of the most conspicuous features in the skull at this stage, and consists of two symmetrical halves. The basitemporals have not at this stage a very firm connection with the base of the skull, but shortly they become firmly united with it and with one another, and thus the pituitary space becomes entirely closed over below by bone.

16. During the previous stage, most of the bones of the face and skull had begun to be ossified, so that during the next or fourth stage, that is about the end of the second and the beginning of the third week of incubation, the chief changes consist in the progressive ossification of the various bones. Since it is not our purpose to enter fully into the structure of the skull of the fowl but rather to confine ourselves to its early development, this and the succeeding stages will be described with greater brevity than the first three.

17. One most important change which occurs during this stage, is the fenestration of the ethmo-presphenoid cartilage. This cartilage (Fig. 70,ps) formed during the second stage an unbroken plate, but towards the end of the third and 
the beginning of the fourth stages, two fenestræ appear. The hindermost of these separates the presphenoid from the ethmoid; but since the hole does not extend to the bottom of the plate, the basi-sphenoid and the ethmoid are continuous below. The other fenestra separates the ethmoid from the nasal septum.

The early condition of the ethmoido-nasal cartilage resembles, as Parker points out, its permanent condition in the Struthiadce, while the condition which it acquires during this stage resembles its condition in Tinamine birds, seeming to prove that the Gallinaceous birds passed through these two stages before reaching the present condition. This plate of cartilage remains during this stage quite unossified. The aliethmoid, aliseptal, and alinasal cartilages which grow out from it on each side, are now severally separated from each other by slight grooves.

18. The chief new ossifying centres which appear in the primordial skull in this stage are :-

(1) An ectosteal plate for the prootic.

(2) 'Two ectosteal plates which appear in each alisphenoid, one in the upper corner, and another immediately above the foramen ovale.

The centres which had begun during the last stages have now spread considerably.

The basioccipital is to a great extent ossified, but still encloses the remains of the notochord. The occipital condyle is however still unossified. Both the exoccipital and supraoccipital plates are spreading rapidly, and the latter have nearly reached the middle line. The quadrate is nearly entirely ossified, but its upper and lower condyles and orbital process are still cartilaginous.

19. The rostrum is still separate from the ethmoid, but has to a great extent coalesced with the basisphenoid. Between the posterior end of the pterygoid and the rostrum, a plate of cartilage is interposed, called the basipterygoid.

The basitemporals have coalesced to a great extent with the osseous outgrowths of the basisphenoid, but at the sides a space is left between the two, through which the Eustachian tubes pass. Between the basitemporals themselves, where they have not coalesced, another space is left, which 
becomes part of the common vestibule in which the Eustachian tubes meet in the middle line.

One new splint bone, the vomer, begins to be formed during this stage in the middle line about half way along the palatines.

The various membrane-bones of the skull are now much more firm and consistent, and the frontals have sent down a process which upon reaching the ethmoidal plate at once sets up ossification in it.

20. In the next stage, about the second day after birth, a considerable number of changes have taken place. The ethmoid has begun to be ossified by two ectosteal plates, one on each side, and the ossification set up by the frontal process in the ethmoid plate has increased. The ethmoid is now only connected with the septum nasi by a narrow isthmus above.

The septum still retains its anterior process, the prenasal cartilage, but this is rapidly diminishing.

In the ear there are now three ectosteal ossifications, the prootic, which appeared in the last stage, and which is by far the largest of the three; the opisthotic, which lies between the prootic and the exoccipital, but is distinct from both; and a third small ectosteal plate, the pterotic.

A small ring-like ossification appears in the internal angular process of Meckel's cartilage; its other process rapidly diminishes and soon disappears.

The supraoccipital ossifications have united in the middle line; the exoccipitals have increased very much, but are quite separate from the opisthotics.

The basioccipital ossification has begun to spread into the condyles.

21. The chief facts of importance in reference to the splint bones, are, that the premaxillaries (Fig. 71, px) have united in the middle line; the vomer has considerably increased in size; the lacrymals have developed, and possess a large supraorbital and a thick anteorbital plate. 'Ihere is still a large fontanelle above, between the frontals and parietals. The squamosals are very large. In the mandible all the splint bones have by this time become developed.

At about the third week after birth two new centres appear, one at the outside of the alisphenoid, forming the centre for the post-frontal, and another over the posterior 
semicircular canal, for the epiotic, which is for a short time distinct from the exoccipital.

In chicks of about two months old, the sutures have begun to lose their distinctness, but two new pairs of centres of ossification appear adjacent to the presphenoid. These first ossify independently, but afterwards set up ossification in the presphenoid, which is still unossified. In fowls of from seven to nine months, the splint bones are beginning to coalesce for the most part with the other bones of the skull, and the sutures are rapidly being obliterated. It would be beyond the scope of this work to enter into the changes by which the skull of the young chick, with most of the sutures distinct, developes into the skull of the adult, where the lines of junction of most of the bones are quite undistinguishable. We shall therefore conclude by giving a table of those bones which are preformed in cartilage, and of the purely splint or membrane bones.

Parts of the bird's skull which are either preformed in cartilage or remain cartilaginous.

Supraoccipital. Exoccipital. Basioccipital. Epiotic. Prootic. Opisthotic. Pterotic. Alisphenoid. Basisphenoid. Orbitosphenoid. Presphenoid. Ethmoid. Post-frontal. Septum nasi, turbinals, prenasal and nasal cartilages. Skeleton of the second and third visceral arches and stapes. Meckel's cartilage and quadrate (first visceral arch). Pterygoid and palatine (superior maxillary process).

Splint-bones not preformed in cartilage.

Parietals. Squamosals. Frontals. Lacrymals. Nasals. Premaxillæ. Maxillæ. Maxillo-palatines. Vomer. Jugals. Quadrato-jugals. Dentary and Bones of Mandible. Basitemporal and rostrum. 


\section{APPENDIX.}

PRACTICAL INSTRUCTIONS FOR STUDYING THE DEVELOPMENT OF THE CHICK.

\section{Incubators.}

OF all incubators, the natural one, i.e. the hen, is by far the best. The number of eggs which fail to develope is much fewer than with an artificial incubator, and the progress of development is much more regular. Under the hen an egg taken, say at 36 hours, will most probably be found in the stage which we have described as proper to that date ; in an artificial incubator, it is nearly sure not to be so. A good sitter will continue to sit for thirty or more days at least, even though the eggs are daily being changed. She should never be allowed to want for water, and should be well supplied according to her appetite with soft food. It is best to place the food at some little distance from the eggs, in order that the hen may leave the eggs when feeding. She will sit most persistently in a warm, quiet, somewhat darkened spot. When an egg is placed under her, the date should be marked on it, in order that the duration of its incubation may be exactly known. When the egg is intended to remain for some time, e.g. for seven days or more, the mark should be bold and distinct, otherwise it will be rubbed off.

Where a "broody" hen cannot be obtained, recourse must be had to an artificial incubator. We have ourselves been so accustomed to employ a hen, that we have very little experience of the various machines which have been introduced as incubators. We have, however, obtained tolerably satisfactory results with an ordinary chemical double-jacketed drying water-bath, thoroughly covered in with a thick coat of cotton wool and flannel baize, and heated by a very small 
gas-jet. If the vessel be filled with hot water, and allowed to cool down to $40^{\circ}$ or thereabouts, before the eggs are introduced, a very small gas flame will be sufficient to maintain the requisite temperature. A small pin-hole-nozzle, giving with ordinary pressure an exceedingly narrow jet of flame about two inches high, is the most convenient. By turning the gas off or on, so as to reduce or increase the height of the jet as required, a very steady mean temperature may be maintained. A simple arrangement of this kind on the whole works more satisfactorily than any of the complicated instruments which have been introduced for similar purposes.

In the absence of gas, a patent night-light placed at a proper distance below the bath may be made to answer very well. When a body of water, once raised to the necessary temperature, is thoroughly surrounded with non-conducting material, a very slight constant amount of heat will supply all the loss.

The temperature should be from $37^{\circ}$ to $40^{\circ} \mathrm{C}$; it may rise temporarily a few degrees above $40^{\circ}$ without any permanent harm, and should not be allowed to fall below $37^{\circ}$.

The eggs within the bath should be placed on and covered up with cotton wool; and the products of the combustion of the gas should be kept as much from them as possible.

\section{Examination of a 36 to 48 hours embryo.}

The student will find it by far the best plan to begin with the study of an embryo of this date. The manipulation is not difficult; and the details of structure are sufficiently simple to allow them to be readily grasped. Earlier embryos are troublesome to manage until some experience has been gained; and the details of later ones are so many as to render it undesirable to begin with them.

A. Opening the egg.

Take the egg warm from the hen or the incubator, and place it (it does not matter in what position, since the blastoderm will at this stage always be found at the uppermost part of the egg) in a small basin large enough to allow the egg to be covered with fluid. It is of advantage, but not necessary, to place 
at the bottom of the basin a mould, e.g. a flat piece of lead with a concavity on the upper surface, in which the egg may rest securely without rolling. Pour into the basin so much of a .7 .5 per cent. solution of sodium chloride warmed to $35^{\circ}$ as will cover the egg completely. With a sharp tap break through the shell at the broad end over the air-chamber, and let out as much air as has already been gathered there. Unless this is done, the presence of air in the air-chamber will cause the broad end to tilt up. At this date there will be very little air, but in eggs of longer incubation, inconvenience will be felt unless this plan be adopted.

Instead of being broken with a blow, the shell may be filed through at one point, and the opening enlarged with the forceps; but a little practice will enable the student to use the former and easier method without doing damage.

With a blunt pair of forceps, remove the shell carefully bit by bit, leaving the shell-membrane behind; begin at the hole made at the broad end, and work over the upper part until about a third or half of the shell has been removed.

Then with a finer pair of forceps remove the shellmembrane; it will readily come away in strips, torn across the long axis of the egg in a somewhat spiral fashion. The yolk and embryo will now come into view.

It is the practice of some simply to break the egg across and pour the yolk and white together into a basin, very much as the housewife does. We feel sure, however, that the extra trouble of the method we have given will be more than repaid in the results.

During this time, and indeed during the whole period of the examination of the embryo in situ, the basin and its contents must be maintained, either by renewal of the salt solution, or by the basin being placed on a sand-bath, at about $38^{\circ} \mathrm{C}$.

\section{B. Examination of the blastoderm in situ.}

This may be done with the naked eye, or with a simple lens of low power. Observe :- 
1. Lying across the long axis of the egg, the pellucid area, in the middle of which the embryo may be obscurely seen as a white streak.

2. The mottled vascular area, with the blood-vessels just beginning to be formed.

3. The opaque area spreading over the yolk with the changes in the yolk around its periphery.

4. (With a simple lens), the contractions of the heart; perhaps the outlines of the head of the embryo may be detected.

\section{Removal of the embryo.}

Plunge one blade of a sharp fine pair of scissors through the blastoderm, just outside the outer margin of the vascular area, and rapidly carry the incision completely round. Until the circle is complete, avoid as much as possible any agitation of the liquid in the basin.

With a little trouble, the excised blastoderm may now be floated into a watch-glass, care being taken to keep it as flat as possible. With a pair of forceps or with a needle, aided by gentle shaking, remove the piece of vitelline membrane covering the blastoderm.

If any yolk adheres to the blastoderm, it may with a little gentle agitation easily be washed off. Sometimes it is of advantage to suck up the yolk with a glass syringe, replacing the fluid removed with clean ( 75 p. c.) salt solution.

The blastoderm should now be removed from the watch-glass to a microscopic glass slide; since it is difficult in the former to prevent the edges of the blastoderm from curling up.

The transference may easily be effected, if both the watch-glass and slide are plunged into a basin of clean warm salt solution. With a little care, the blastoderm can then be floated from the one to the other, and the glass slide, having the blastoderm with its upper surface uppermost spread flat upon it, very gently raised out of the liquid. 
A thin ring of putty may now be placed round the blastoderm, a small quantity of salt solution gently poured within the ring, and the whole covered with a glass slide, which may be pressed down until it is sufficiently close to the embryo. The presence of any air-bubbles must of course be avoided.

Provided care be otherwise taken to keep the embryo well covered with liquid, the putty ring and the coverslip may be dispensed with. They are often inconvenient, as when the embryo has to be turned upside down.

The object is now ready for examination with a simple lens or with a compound microscope of low objective. It is by far the best for the student to begin at least with the simple lens. In order that everything may be seen at its best, the slide should be kept warmed to about $38^{\circ}$, by being placed on a hot stage.

\section{Surface view of the transparent ambryo from above.}

The chief points to be observed, are :-

1. The head-fold.

2. The indications of the amnion; especially the false amnion, or outer amniotic fold.

3. The neural tube: the line of coalescence of the medullary folds, the first cerebral vesicle, the commencing optic vesicles, the indications of the second and third cerebral vesicles, the as yet open medullary folds at the tail end.

4. The heart seen dimly through the neural tube; note its pulsation if present.

5. The fold of the somatopleure anterior to the heart (generally very faintly shewn).

6. The fold of the splanchnopleure (more distinctly seen) : the omphalo-mesaraic veins.

7. The protovertebrce. 
8. Indications of the omphalo-mesaraic arteries.

9 . The as yet barely formed tail-fold.

10. The commencing blood-vessels in the pellucid and vascular areas.

E. Surface view of the transparent embryo from below.

The coverslip must now be removed and the glass slide again immersed in a vessel of clean salt solution. By gently seizing the extreme edge of the opaque area with a pair of forceps, no difficulty will be found in so floating the blastoderm, as to turn it upside down, and thus to replace it on the slide with the under surface uppermost.

The points which most deserve attention in this view, are :-

1. The heart: its position, its union with the omphalomesaraic veins, its arterial end.

2. The fold of the splanchnopleure marking the hind limit of the gut; the omphalo-mesaraic veins running along its wings.

3. The protovertebrce on each side of the neural canal behind the heart; farther back still, the vertebral plates not divided into protovertebræ.

F. The cxamination of the cmbryo as an opaque
object.

This should never be omitted. Many points in the transparent embryo only become intelligible after the examination of it as an opaque object.

Having removed the putty ring and coverslip, if previously used, allow the blastoderm so far to become dry, that its edge adheres to the glass slide. Care must of course be taken that the embryo itself does not become at all dry. Place the glass slide with the blastoderm extended flat on it, in a shallow vessel containing a $\cdot 1$ or $\cdot 5$ per cent. solution of chromic acid. 
If the blastoderm be simply immersed by itself in the chromic acid solution, the edges of the opaque area will curl up and hide much of the embryo. The method suggested above prevents these inconveniences.

The embryo thus hardened and rendered opaque by immersion in chromic acid, (a stay of 24 hours in the solution will be sufficient) may be removed to a watch-glass, containing either some of the solution, or plain water, and examined with a simple lens, under a strong direct light. The compound microscope will be found not nearly so advantageous for this purpose as the simple lens. A piece of black paper placed under the watch-glass, will throw up the lights and shadows of the embryo, with benefit. The watch-glass should have a flat bottom; or a shallow flat glass cell should be used instead.

a. Looking at the embryo from above, observe:-

1. The head-fold; the head distinctly projecting from the plane of the blastoderm, and formed chiefly by the forebrain and optic vesicles.

2. The elevation of the medullary canal, and the indications of the side walls of the embryo.

3. The indications of the tail.

4. The Amnion partly covering the head. Tear it open with needles. Observe its two folds.

b. Having turned the blastoderm upside down, observe the following points, looking at the embryo from below.

1. The hinder limit of the splanchnopleure in the headfold, marking the hind limits of the fore-gut. The opaque folds now conceal the head almost entirely from view.

2. The commencing tail-fold, and the shallow boatshaped cavity (of the alimentary canal) between it and the head-fold.

The student should not fail to make sketches of 
the embryo, both as a transparent, and as an opaque object, seen from below as well as from above. These sketches will be of great service to him when he comes to study the sections of the same embryo.

\section{G. On preparing sections of the embryo.}

1. Hardening.

a. With chromic acid.

The embryo must be immersed in the way recommended under $\mathrm{F}$, in a solution of the strength of " 1 p.c. for 24 hours. From this it should be removed and placed in a stronger solution ( 3 p. c.) for another 24 hours. If it then appears sufficiently hard, it may be at once placed in alcohol of $70 \mathrm{p}$. c., in which it should remain for one day, and then be transferred to alcohol of 90 p.c., after remaining in which for two days, it should lastly be placed in absolute alcohol; in this it can stay till required for section. If the chromic acid has not by this time been completely got rid of, the absolute alcohol must be changed, till the specimen is entirely free from acid.

b. With picric acid.

The best form for applying the picric acid, is that introduced by Dr Kleinenberg.

With 100 parts of water, make a cold saturated solution of picric acid; add to this two parts of concentrated sulphuric acid: filter and add to the filtrate three times its bulk of water.

In this solution of picric acid, the embryo must be immersed in the same manner as when chromic acid is used (vide F). After remaining in the acid for five hours, it is to be treated successively with weak, strong and absolute alcohol, as was done in the case of the chromic acid. Still more difficulty will probably be found in getting rid of the picric acid, than was found to 
be the case with the chromic acid, and the alcohol will probably have to be changed several times.

\section{c. With osmic acid.}

Immerse the embryo in a 5 p.c. solution of osmic acid; leave it in this, covered and in the dark, for $2 \frac{1}{2}$ hours, and then place the embryo in absolute alcohol, taking care completely to get rid of the acid by washing several times with alcohol.

The osmic acid method has the advantage of being simpler than the others, and also of leaving the cells in a more natural condition. Its disadvantages are, that it is less certain, and further, that it is necessary to cut sections of the embryo the next day after it has been placed in the spirit. Otherwise it becomes too brittle; the sections are then not so easy to make, and not so good. It has the further disadvantage that the outlines of the individual cells are not so clearly brought out as with the other two reagents.

Absolute alcohol has also been employed as a hardening reagent, but is by no means so good as the reagents recommended above.

\section{Stainixg.}

In most cases it will be found of advantage to stain the embryo. The best method of doing this, is to stain the embryo as a whole, rather than to stain the individual sections after they have been cut.

Either carmine or hæmatoxylin may be employed. For carmine, Beales' or some other alcoholic solution is the best. Into this the embryo may be removed directly from the absolute alcohol, left in the carmine for 24 hours, and then placed again in absolute alcohol for another 24 hours before being cut.

The best solution of hæmatoxylin, one for which 
we are indebted to Dr Kleinenberg, is made in the following way.

1. Make a saturated solution of crystallized calcium chloride in 70 p.c. alcohol, and add alum to saturation.

2. Make also a saturated solution of alum in 70 p.c. alcohol, and add 1 to 2 in the proportion of $1: 8$.

3. To the mixture of 1 and 2 add a few drops of a barely alkaline saturated solution of hæmatoxylin.

The embryo may be removed into this directly from the absolute alcohol, left in it for $\check{5}$ hours and placed back again in absolute alcohol for 24 hours.

Neither the carmine nor the hæmatoxylin will stain if the embryo is not quite free from acid.

Only the chromic or picric acid specimens require to be stained. It is however possible to stain the osmic acid specimens with hæmatoxylin.

\section{Imbeding.}

It is hardly possible to obtain satisfactory sections of embryos, without employing the method of imbedding now so largely employed in histological studies.

The substances most generally used, are:-

1. Paraffin, obtained by heating together five parts of solid paraffin with one part of paraffin oil and one part of pig's lard.

2. Wax and oil, made by heating together three parts of common white wax and one of olive-oil.

3. Spermaceti, obtained by heating together very thoroughly four parts of spermaceti and one of cocoa butter, or four parts of spermaceti and one of castor oil.

[4. Gum. The above substances are all liquid when warm, and become solid when cold. When a solution of gum is used as an imbedding medium, 
it must be hardened with alcohol. We cannot however recommend this substance for embryos. For the method of employing it, see Hand-book for the Physiological Laboratory, p. 92.]

The exact consistency of these various mixtures should be made to vary according to the solidity of the embryo, by increasing or diminishing the various constituents.

From a cold solid mass of one of the mixtures, cut a cubical block (about an inch cube), and on one surface make a shallow cavity, large enough to receive the blastoderm. Having with a morsel of blotting-paper removed all superfluous alcohol from the blastoderm place it quite flat in the cavity just made, and gently pour over it some of the mixture sufficiently heated to have become liquid (care being taken, of course, that it is not too hot). With a heated needle, remove any bubbles that may make their appearance; and make on the block, as a guide in cutting the sections, a mark of the exact position of the embryo. It is sometimes of advantage to transfer the embryo from the alcohol to some oil of cloves (when the wax and oil is used, or to some creosote, when the paraffin is employed), and to allow it to become saturated with that substance, before placing it in the block. The adbesion of the imbedding material to the object imbedded, is thus rendered more complete.

This method serves fairly well, when there are no cavities in the object into which it is necessary for the mixture to penetrate. When these exist, the following method (again Dr Kleinenberg's) will be found the most satisfactory.

Remove the object from the absolute alcohol and place it directly into Bergamot oil, and leave it there, till the oil has completely penetrated through it. In the mean time, prepare a small paper box, by bending up the sides and folding in the corners of a piece of stiff paper, and pour on the bottom of it a thin coating of the mixture of spermaceti and castor oil. When this has become solid, place the 
embryo flat upon it, having removed as much superfluous Bergamot oil as possible, and then pour in some more of the spermaceti mixture, which should be as hot as can be used without injuring the object. With a hot needle, move the object about so as to get rid of all air bubbles, and to assist the spermaceti in penetrating all parts of the embryo. Finally, before the spermaceti becomes solid, place the embryo in the position required for subsequently making sections; and when the whole becomes solid, make a mark over the position of the embryo. It is better to soak the object in the hot spermaceti before finally imbedding it, but the manipulation of this is rather difficult with embryos of this age.

If successfully imbedded, the spermaceti will be found to have penetrated through and through the embryo; and the method has the great merit of permitting the imbedding medium to be quite easily dissolved away from the sections, by a few minutes' immersion in a mixture of four parts of turpentine to one of creosote. With the other imbedding materials of which we have spoken, this cleaning of the sections is very troublesome and difficult, and liable to result in injury to the specimens.

\section{Cutting Sections.}

When the imbedding block is cold pare away the edges and then gradually slice it away until the edge of the area opaca appears. The sections must then be made more carefully, and each one examined till the actual body of the embryo is arrived at.

It is best to begin with transverse vertical sections which may commence either at the tail or head end. 'The latter is preferable, since the bit which is last cut is apt to slip.

A section instrument may be used ; but we ourselves very much prefer a simple razor provided with heavy fixed handle (i.e. with no hinge) held in the hand. 
In any case the cutting-blade should for the first method of imbedding be kept freely wetted with spirit; and each section, as it is made, carefully floated on to a glass slide and then, the spirit having been removed, mounted in glycerine, or treated with creosote or oil of cloves and turpentine and mounted in balsam or dammar. It is well to guard the section with a small diaphragm of paper placed under the cover-slip.

When the object has been imbedded in spermaceti in the way recommended above, the block of spermaceti and the razor should be moistened with olive oil and not with spirit. The sections must be floated from the razor on to the glass slip in the ordinary way and then treated with a mixture of turpentine ( 4 parts) and creosote (1 part) till all the oil and the spermaceti are removed. They may then be mounted in Canada balsam or dammar in the usual way. Even when the other methods of imbedding spoken of above are adopted, it will frequently be found advantageous to moister the razor with creosote or oil of cloves rather than with spirit.

Whichever method be followed, a series of sections, each as thin as possible, should be obtained and carefully numbered, from head to tail or vice versa. They should at first at least all be retained for study, and not even the fragmentary ones thrown away, these being often the most instructive.

The following transverse sections will perhaps be the most instructive.

1. Through the optic vesicles, shewing the optic stalks.

2. Through the hind-brain, shewing the auditory sacs.

3. Through the middle of the heart, shewing its relations to the splanchnopleure and alimentary canal.

4. Through the point of divergence of the splanchnopleure folds, shewing the venous roots of the heart.

5. Through the dorsal region, shewing the medullary 
canal, protovertebræ and commencing cleavage of the mesoblast.

6. Through a point where the medullary canal is still open, shewing the mode in which its closing takes place.

A good series of transverse sections having been obtained, longitudinal vertical ones may be made in a similar manner. These are however much more difficult to manage; and are moreover chiefly of use to compare with the transverse ones.

GG. Preservation of the embryo as a whole.

Embryos of this or an earlier day may be easily preserved whole as microscopic objects; but the value of such preparations is very slight, and they are perhaps not worth the trouble. The best method is probably to place the embryos in the picric acid solution for a short time, and then successively in weak, strong and absolute alcohol and finally to mount them, in glycerine or, after staining and treatment with oil of cloves, in balsam. For shewing some points, treatment with osmic acid for a short time and then with alcohol and finally with glycerine may be adopted.

Whole embryos of a later date cannot be satisfactorily preserved as microscopic objects.

\section{Examination of an Embryo of about 48-50 hours.}

A. Opening the egg-as in II. A.

B. Examination of the blastoderm in situ. Observe

1. The form of the embryo, which is much more distinct than at the earlier stage.

2. The beating of the heart.

3. The general features of the circulation. 
C. Removal of the embryo from the yolk, as in
II. C.

D. Surface view of the transparent cmbryo from above.

Notice:-

1. General form of the embryo.

a. Commencing cranial flexure.

b. The tail and side folds.

2. Ammion. Notice the inner and outer (false amnion) limbs and remove them with a needle. When the amnion has been removed the features of the embryo will be much more clearly visible.

3. The organs of sense.

a. Eye. Formation of the lens already nearly completed.

6. Auditory involution, now a deep sac with a narrow opening to the exterior.

4. The brain.

a. The vesicles of the fore-, mid-, and hind-brain.

b. The two cerebral lobes, which have been budded off from the fore-brain.

c. The cranial flexure taking place at the midbrain.

E. Transparent cmbryo from below.

Manipulation as in II. E.

Notice:-

1. The increase of the head-folds of the somatopleure and splanchnopleure, especially the latter, and the commencement of these folds at the tail. 
2. The now $\boldsymbol{\Omega}$-shaped heart; for further particulars vide Chap. IV. $\S 18$.

3. The commencing 1 st and 2 nd visceral clefts and the aortic arches.

4. The circulation of the yolk sac, vide Fig. 23. Make out all the points there shewn and ascertain by examination that what have been called the veins and arteries in that figure, are truly such.

F. The embryo as an opaque object. Treatment as in II. F.

From above:

Observe the amnion, which is a very conspicuous object, and remove it with needles if not done previously. The external form of the brain and the auditory sac appear very distinctly.

From BeLOW:

Observe the nature of the head-and tail-folds, which are much more easily understood from the opaque than from the transparent embryos.

Observe also the alimentary canal, the widely open hind end of the fore-gut, and the front end of the as yet very short hind-gut.

\section{G. Scctions.}

Manipulation as in II. G. The embryo can still be stained as a whole.

The more important sections to be preserved, are

1. Through optic lobes, shewing:

a. The formation of the lens.

b. The involution of the primary optic vesicle.

c. The constriction, especially from above, of the optic stalk. 
2. Through auditory sac, shewing:

a. Auditory sac still open.

b. The thin roof and thick sides of the hind-brain.

c. Notochord.

d. Heart.

e. Closed alimentary canal.

3. Through dorsal region, shewing the general appearance of a section of an embryo at this stage, which should be compared with a similar section of the earlier stage.

It shews:

a. The commencement of the side folds; the alimentary canal still however open below.

b. The Wolffian duct Jying close under the epiblast on the outside of the protovertebræ.

c. The notochord with the aortæ on each side.

IV. Examination of an Embryo at the end of the third day.

A. Opening the egg, as in II. A.

B. Examination of the blastoderm in situ. Observe:-

1. The great increase of the vascular area both in size and distinctness. The circulation is now better seen in situ than after the blastoderm has been removed.

2. That the embryo now lies completely on its left side and that it is only connected with the yolk-sac by a somewhat broad stalk.

C. Removal of the embryo. See II. C.

It is now unnecessary to remove the whole of the blastoderm with the embryo; indeed it is better to 
cut away the vascular area unless it is wanted for examination.

\section{Surface view of the transparent embryo.}

Since the embryo now lies on its side we shall not have to speak of the view from above and below. The riews from the two sides differ chiefly as to the appearance of the heart.

The embryo (freed from the blastoderm and the amnion) is to be floated on to a glass slide in the usual way. It is necessary to protect it while under examination, with a cover-slip, which must not be allowed to compress it. To avoid this, we have found it a good plan to support the cover-slip at one end only, since by moving it about when thus supported, a greater or less amount of pressure can be applied at will to the object.

The details which can at this stage be seen in a transparent embryo are very numerous and we recommend the student to try and verify everything shewn in Fig. 24. Amongst the more important and obvious points to be noticed are

1. The increase of the cranial flexure and the bodyflexure.

2. The condition of the brain. The mid-brain now forms the most anterior point of the head.

The fore-brain consists of the inconspicuous vesicle of the third ventricle and the two large cerebral lobes.

The hind-brain consists of a front portion, the cerebellum with a thickened roof; and a hinder portion, the fourth ventricle with a very thin and delicate roof.

3. Organs of sense.

The eye especially is now in a very good state to observe. The student may refer to Fig. 31, and the description there given. 
The sar-vesicle will be seen either just closing or completely closed.

4. In the region of the heart attention must also be paid to:

a. The visceral clefts.

b. The investing-mass, i.e. the growth of mesoblast taking place around the end of the notochord.

c. The condition of the heart.

5. In the region of the body the chief points to be observed are:

a. The increase in the number of the protovertebrce.

b. The Wolffian duct, which can be seen as a streak along the outer side of the hinder protovertebræ.

c. The allantois, which is now a small vesicle lying between the folds of the somatopleure and splanchnopleure at the hind end of the body, but as yet hardly projects beyond the body cavity.

F. The cmbryo as an opaque object.

Preparation as in II. F.

The general form of the embryo can be very satisfactorily seen when it is hardened and examined as an opaque object; but the most important points to be made out at this stage in the hardened specimens are those connected with the visceral clefts and folds and the mouth.

If the amnion has not been removed it will be necessary to pick it completely away with needles. Without further preparation a view of the risceral folds and clefts may be obtained from the side; but a far more instructive view is that from below, in order to gain which the following method may be adopted.

Pour a small quantity of melted black wax (made by mixing together lampblack and melted wax) into a watch-glass, using just enough to cover the bottom of the glass. While still soft make a small depression in the wax with the rounded end of a pen-holder or handle of a paint-brush and allow the wax to cool. 
In the meantime cut off the head of the hardened embryo by a sharp clean transverse incision carried just behind the visceral clefts, transfer it to the watchglass and cover it with water or spirit. By a little manipulation the head of the embryo may now be shifted into the small depression in the wax, and thus be made to assume any required position. It should then be examined with a simple lens under a strong reflected light, and a drawing made of it.

When the head is placed in the proper position, the following points may easily be seen.

1. The opening of the mouth bounded below by the first pair of visceral folds, and commencing to be enclosed above by the now very small buds which are the rudiments of the superior maxillary processes. Compare Fig. 38.

2. The second and third visceral arches and clefts.

3. The nasal pits.

\section{G. Sections. Manipulation as in II. G.}

The embryo can still be stained as a whole.

The most important sections are:-

1. Through the eyes in the three planes, vide Fig. 30, D. E. F.

2. Through the auditory sac.

3. Through the dorsal region, shewing the general changes which have taken place.

Amongst these, notice

a. The changes of the protovertebrce: the commencing formation of the muscle-plates.

b. The position of the Wolffian duct and the formation of the germinal epithelium.

c. The aortce and the cardinal veins.

d. The great increase in depth and relative diminution in breadth of the section. 


\section{Examination of an Embryo of the Fourth Day.}

A. Opening the egg, as in II. A.

Great care will be required not to injure the blastoderm, which now lies close to the shell-membrane.

B. Examination in situ. Observe:-

1. The now conspicuous amnion.

2. The allantois, a small, and as yet hardly vascular vesicle, beginning to project from the embryo into the space between the true and the false amnion.

3. The rapidly narrowing somatic stalk.

C. Removal of the cmbryo, as in II. C. and IV. C.

The remarks made in the latter place apply with still greater force to an embryo of the fourth and succeeding days.

D. Surface view of the transparent embryo. For manipulation, vide IV. D.

The points to be observed are:-

1. The formation of the fifth, seventh, and ninth cranial nerves.

To observe these, a small amount of pressure is advantageous.

2. The formation of the fourth visceral cleft, and the increase in size of the superior maxillary process.

3. The formation of the nasal pits and grooves.

4. The great relative growth of the cerebral lobes and the formation of the pineal gland from the roof of the vesicle of the third ventricle.

5. The great increase in the investing mass.

6. The formation and growth of the muscle-plates, which can now be easily seen from the exterior.

7. The allantois. Make out its position and mode of opening into the alimentary canal. 
F. The embryo as an opaque object. Manipulation as II. F. For mode of examination vide IV. F.

The view of the mouth from underneath, shewing the nasal pit and grooves, the superior and inferior maxillary processes and the other visceral folds and clefts, is very instructive at this stage. Compare Fig. 48.

\section{G. Sections. Manipulation as in II. G.}

A slightly stronger solution of chromic acid may be used than for the younger embryos.

The student will most probably find that he can still stain the embryo as a whole, especially if he employs hæmatoxylin. If this cannot be clone, the sections must be stained individually after being cut. The most important sections are,

1. Through the eyes.

2. Transverse section immediately behind the visceral arches, shewing the origin of the lungs.

3. Transverse section just in front of the umbilical stalk, shewing the origin of the liver.

4. Transverse section at about the centre of the dorsal region, to shew the general features of the fourth day. Compare Fig. 47.

Amongst the points to be noticed in this section, are a. Muscle-plates.

b. Spinal nerves and ganglia.

c. Wolffian duct and bodies.

d. Miiller's duct.

e. Mesentery.

$f$. Commencing changes in the spinal cord.

5. Section passing through the opening of the allantois into the alimentary canal.

For the points to be observed in embryos of the fifth and sixth days, the student must consult the chapters devoted to those days. 
In the hardened specimens, especial attention should be paid to the changes which take place in the parts forming the boundaries of the mouth.

In making sections, it will probably be found impossible to stain the embryo as a whole; in this case, the individual sections must be stained separately.

\section{Examination of a Blastoderm of 20 hours.}

A. Opening the cgg, as in II. A.

B. Examination in situ.

It will not be found possible to make out anything very satisfactory from the examination of a blastoderm in situ at this age. The student will however not fail to notice the halones, which can be seen forming concentric rings round the blastoderm.

C. Removal of the embryo.

Two methods of hardening can be adopted at this age. One of these involves the removal of the blastoderm from the yolk, as in II. C. In the other case, the yolk is hardened as a whole. The latter of these is the most satisfactory method for sections; but if employed, the embryo cannot be viewed as a transparent object.

In the cases where the blastoderm is removed from the yolk, the manipulation is similar to that described under II. C, with the exception of more care being required in freeing the blastoderm from the vitelline membrane.

D. Surface view transparent, from above. Observe:-

1. The medullary groove between the two medullary folds, whose hind ends diverge to enclose between them the end of the primitive groove.

2. The head-fold at the end of the medullary groove.

3. The one or two pairs of protovertebrce flanking the medullary groove. 
4. The notochord as an opaque streak along the floor of the medullary groove.

E. Surface view transparent, from below.

Same points to be seen as from above, but less clearly.

F. Embryo as an opaque object.

As an opaque object, whether the embryo is hardened in situ or after being removed from the yolk, the same points are to be seen as when it is viewed as a transparent object, with the exception of the notochord and protovertebræ (vide D). The various grooves and folds are however seen with far greater clearness.

\section{G. Sections.}

Two methods of hardening may be employed; (1) with the embryo in situ, (2) after it has been removed.

To harden the blastoderm in situ the yolk must be hardened as a whole. After opening the egg either leave the yolk in the egg-shell or pour it out into a Berlin capsule; in any case freeing it as much as possible from the white, and taking especial care to remove the more adherent layer of white which immediately surrounds the yolk.

Place it in a weak solution of chromic acid (first of 11 p.c. and then of 5 p.c.) with the blastoderm uppermost and leave it in that position for two or three days.

Care must be taken that the yolk does not roll about; the blastoderm must not be allowed to alter its position: otherwise it may be hard to find it when everything has become opaque. If at the end of the second day the blastoderm is not sufficiently hard, the strength of the solution should be increased and the specimen left in it for another day.

After it has become hardened by the chromic acid, the yolk should be washed with water and treated successively with weak and strong spirit, vide II. G. After it has been in the strong spirit (90 p. c.) for two days, the vitelline membrane may be safely peeled off 
and the blastoderm and embryo will be found in situ. The portion of the yolk containing then must then be sliced off with a sharp razor, and placed in absolute alcohol.

The staining, \&c. may be effected in the ordinary way.

If osmic acid, which we believe will be found especially serviceable for these early stages, is employed, it will be necessary to remove the blastoderm from the yolk before treating it with the reagent.

The following transverse sections are the most important at this stage:

1. Through the medullary groove, shewing

a. The medullary folds with the thickened mesoblast.

b. The notochord under the medullary groove.

c. The commencing clearage of the mesoblast.

2. A section through the region where the medullary folds diverge, to enclose the end of the primitive groove, shewing the greatly increased width of the medullary groove, but otherwise no real alteration in the arrangement of the parts.

3. A section through the front end of the primitive groove with the so-called axis cord underneath it, while on each side of it are still to be seen the medullary folds.

4. A section through the primitive groove behind this point, shewing the typical characters of the primitive groove.

VII. Examination of an unincubated Blastoderm.

A. Opening the egg. Vide II. A.

B. Examination of the blastoderm in situ.

Observe the central white spot and the peripheral more transparent portion of the blastoderm and the halones around it. 
C. Removal of the blastoderm. Vide VI. C.

With the unincubated blastoderm still greater care is required in removal than with the 20 hours' blastoderm, and there is no special advantage in doing so unless it is intended to harden it with osmic acid.

D. Surface view transparent from above.

Observe the absence of the central opacity.

E. Surface view transparent from underneath. Nothing further to be observed than from above.

F. As an opaque object.

There is nothing to be learnt from this.

G. Sections.

Manipulation as in VI. G.

Only one section is required, viz. one taken through the centre of the blastoderm, shewing:

a. The distinct epiblast.

$b$. The lower layer cells not as yet differentiated into mesoblast and hypoblast.

c. The thickened edge of the blastoderm.

d. The segmentation cavity and formative cells.

\section{Examination of the process of Segmentation.}

To observe the process of segmentation it will be found necessary to kill a number of hens which are laying regularly. The best hens lay once every 24 hours, and by observing the time they usually lay (and they generally lay pretty regularly about the same time), a fair guess may be made beforehand as to the time the egg has been in the oviduct. By this means a series of eggs at the various stages of segmentation, may usually be obtained without a great unnecessary sacrifice of hens. For making sections, the yolk must in all cases be hardened as a whole, which may be done as recommended in VI. G. 
Chromic acid is an excellent reagent for this and it will be found very easy to make good sections.

In the sections especial attention should be paid,

1. To the first appearance of nuclei in the segments, and their character.

2. To the appearance of the horizontal furrows.

3. As to whether new segments continue to be formed outside the limits of the germinal disc, or whether the firesh segmentation merely concerns the already formed segments.

4. In the later stages, to the smaller central and larger peripheral segments, both containing nuclei.

For surface views, the germinal disc, either fresh or after it has been hardened, can be used. In both cases it should be examined by a strong reflected light. The chief point to be noticed is the more rapid segmentation of the central than of the peripheral spheres.

\section{Examination of the later changes of the Embryo.}

For the later stages, and especially for the development of the skull and the vascular system of the body of the chick, it will be found necessary to dissect the embryo. This can be done either with the fresh embryo or more advantageously with embryos which have been preserved in spirit.

If the embryos are placed while still living into spirit a natural injection may be obtained. And such an injection is the best for following out the arrangement of the blood-vessels.

Sections of course will be available for study, especially when combined with dissections.

\section{Study of the development of the Blood-vessels.}

Observations on this subject must be made with blastoderms of between $30-40$ hours. These are to be removed from the egg, in the usual way (vide II. A. and C.) spread out over a glass slip and examined from below, vide II. $\mathrm{E}$. 
The blastoderm when under examination must be protected by a cover-slip with the usual precautions against pressure and evaporation, and a hot stage must also be employed.

Fresh objects so prepared require to be examined with a considerable magnifying power (400 to 800 diameters). From a series of specimens between 30 and 40 hours old all the points we have mentioned in Chapter IV. $\S 6$ can without much difficulty be observed.

Especial attention should be paid in the earlier specimens to the masses of nuclei enveloped in protoplasm and connected with each other by protoplasmic processes; and in the later stages to the conversion of these nuclei into blood corpuscles and of the protoplasmic processes into capillaries, with cellular walls.

Blastoderms treated in the following ways may be used to corroborate the observations made on the fresh ones.

\section{With gold chloride.}

Immerse the blastoderm in gold chloride ( 5 p.c.) for one minute and then wash with distilled water and mount in glycerine and examine.

By this method of preparation, the nuclei and protoplasmic processes are rendered more distinct, without the whole being rendered too opaque for observation.

The blastoderm after the application of the gold chloricle should become a pale straw colour; if it becomes in the least purple, the reagent has been applied for too long a time.

2. With potassium bichromate.

Immerse in a 1 p. c. solution for one day and then mount in glycerine.

3. With osmic acid.

Immerse in a $\breve{5}$ p.c. solution for half an hour and then in absolute alcohol for a day, and finally mount in glycerine. 


\section{N D E X.}

\section{A.}

AFdistssiefF, $64,71,72$.

Air-chamber, 12.

Albumen, I3.

Aliethmoid cartilage, 236 .

Alimentary canal, $6 \mathrm{r}$.

Alinasal cartilages, $227,231,236$.

Aliseptal cartilages, $23 \mathrm{I}, 236$.

Alisphenoid, 232.

Allantoic arteries, 168.

Allantoic veins, 210.

Allantois, 4I, I47-I50, I74, 20I, $202,203$.

Amnion, 39-4I, 57, $8_{3}, \mathrm{I}_{4} \mathrm{I}, 20 \mathrm{I}$, 202 ; cavity of, 40.

Amniotic fluid, 4 I ; sac, 4 r.

Annuli fibrosi, I $_{5} 8$.

Anterior fissure, I86, I $8 \%$.

Anus, I83.

Aorta, dursal, I 2 I ; primitive aortæ, 66,79 .

Aortic arches, 8 r, I 2 I, 2 I3, 2 I 7,2 I 8 .

Aquapendente, FAbricius of, 2 .

Aqueductus vestibuli, I I I, I I 5 .

Area opaca, 16, I \&; pellucida, I6.

Aristotle, 2.

Arteriæ collaterales colli, 2 I 7 .

Arterial arches, I68, I69; arterial system, 213-220.

Arteries; allantoic $\mathbf{6 8}$; iliac 168 , $2 \mathrm{I}+$; omphalo-mesaraic $66,80,168$, 202, 214 ; subclavian 217 ; umbilical I68; vertebral 2 I 7 .

Auricles, 63, 79, I73.

Axis-cord, 48 .

B.

BabUchis, io6.

Batr. Sce Vox Bater.

Basibranchial, 230.

Basi-hyal, 230.
Basioccipital, 236.

Basipterygoid, 236 .

Basisphenoid, 231 .

Basisphenoidal ossicles, 235.

Basitemporals, 232, 235,236 .

Beak, 205.

Blastoderm, 5, I4, I6-I8, I7 t, 241 , 242,252 ; of 20 hours, 261 ; unincubated 263 .

Blood-vessels, 66-72; study of development of, 265,266 .

Body-folds, 3 I.

Boettcher, I I 3 , I I 4 , I I 6 .

Bone; metatarsal 176; occipital 227; tarsal, 176 .

BoNNeT, 4 .

BORNHAUPT, I 49, I62.

Branchial clefts, 1 I 9 .

Branchial fold, I 9 .

Bulbus arteriosus, $63,79, \quad 123$, I 73 , I9O-I94, 2 I 4,2 I 5.

C.

Canal; alimentary 6r; semicircular, 115.

Canales Botalli, 2 I 9, 220.

Canalis auricularis, 123,172 ; reuniens, I I I.

Capsules, periotic, 227 .

Cardinal veins, 124 .

Carotids, I69, 2 I 3 .

Carpals, 175,176 .

Carpo-metacarpus, I 75 .

Carpus, I75.

Cartilagre, $230-23^{2}$; aliethmoid $23^{6}$; alinasal $227,231,236$; aliseptal 231 , $23^{6}$; ethmoido-nasal 236 ; ethnopresphenoid 235, 236 ; Meckel's, I 79, I $82,229,230$; paired rods of, 227 ; pre-nasal, 23 I, 232. 
Cartilage-bone, I 78 .

Cartilaginous rods, 228; skeleton, 206 ; skull, $230-233$.

Cavity of the ammion, 40.

Cell-mass, intermediate, $82,136,161$, I 65.

Centrale, 176 .

Cerato-branchial, 230.

Cerato-hyals, 230.

Cerebelium, 93 .

Cerebral vesicles, $58-60$.

Chalaze, I3.

Change of position of embryo, 87 .

Chorion, 32, 42, 203.

Choroid, roz.

Choroidal fissure, 99-10r.

Cicatricula, I 4. See Blastoderm.

Circulation, 79, 81, 168-173, 220223.

Clarke, Lockhart, I53, 185, I87 ; L. C., I 86.

Cleavage of the mesoblast, $38,57,65$, $202,203$.

Clefts; branchial, 119; visceral, II9, 204.

Clinoid walls $23 \mathrm{I}$.

Cochlea, II I, i I2.

Condyle, occipital, $230,233,236$.

Cones, 106.

Coni vasculosi, 167.

Corpora bigemina, 93 .

CORTI, rods of, II 7 .

Coste, 22, I 8 I.

Cranial flexure, $78,8_{7}, 89, \mathbf{1} 43$; cranial nerves, $137,138,147$.

Cranium, I77-I79, 225-238.

Crura cerebri, 93 .

Curvature of the body, 87 .

Cutting sections, $250-252$.

D.

DARESTE, I 5 .

Descemet, membrane of, Io2.

Descent of the ovum, $2 \mathrm{I}$.

Digits, 175 .

Disc, germinal, 19 .

Discus proligerus, I9.

JobRTIN, I25, I48, I49.

Döllinger, 5.

Dorking fowls, 176 .

Dorsal aorta, I 2 I.

Duct of Miuller, I62, 163, 168; umbilical duct, 143,174 . See Wolffian duct.
Ductus arteriosi, 219; Botalli, 219; cochlearis, I I I, I15; Cuvieri, 124, I 7 I ; venosus, $123,170,210$.

Duodenum, 128.

Dursy, 50, 78, 82, 91, г56.

E.

Ear, 78, III 117,237 .

Ectosteal ossification, 233, $23 \%$.

Ectostosis, 233.

Egg-shell, II.

Elbow, 175 .

Embryo as an opaque object, 245 , $254,257,260,262$; of the third day, examination of, $252-258$; of the fourth day, $259-26 \mathrm{r}$.

Embryology, meaning of, I.

Embryonic sac, 35; shield, 44.

Endostosis, 233 .

Epiblast, 27, 44, 56, 197 .

Epididymis, $16 \%$.

Epigenesis, 3.

Epiotic, 238.

Episkeletal muscles, I 59 .

Epithelium, germinal, 160,165 .

Ethmoid, 237.

Ethmoido-nasal cartilage, 236 .

Ethmo-presphenoid cartilage, 235, 236 ; plate, $23 \mathrm{I}$.

Eustachian valve, 195.

Evolution, 3.

Examination of the blastoderm in situ, 24I, 242, 252, 255 .

Exoccipital plates, 236 .

Exoccipitals, 230.

Eye, I 7 .

Eyeball, 94.

F.

FABRICIUS, 2, 3 .

Feathers, 205.

Fenestra ovale, 232 ; rotundum, 232.

Fenestræ, 236.

Fibulare, 176 .

Fissure; anterior, $186, \mathrm{I} 87$; choroidal, 99-10I ; posterior, 187 .

Fotal appendages, 201.

Foot, 175,176 .

Foramen ovale, 194; occipital, $22 \%$.

Fore-brain, $78,9 \mathrm{I}$.

Foregut, 62,

Formative cells, $r_{7}, 26,44$. 
Fourth ventricle, 9t.

Fretum Halleri, 172 .

Frontals, 235 .

Fronto-nasal process, I 20,145 .

\section{G.}

Gall-bladder, 13.3.

Gasserian gangliun, $14 \%$.

Gegenbaur, $15+, 157,158,228,229$.

Genital ridge, 164 .

Germinal disc, 19; epithelium, 160, I 65 ; spot, 20 ; vesicle, 20.

Gland, pineal, 9 I.

Glossopharyngeal nerve, $1+7$.

Goette, 25, 50, 72, 93, 12S, 129 , I3I, $133,1+7$.

Grattian follicle, I66, I67.

Grey column, I $8_{4}$, I 85 ; grey matter, $184,185,186,187,189$.

\section{H.}

Hrmatoxylin, solution of, 247,248 .

Halones, 52 .

HALLER, 4,5 .

Hand, 175,176 .

Hardening, $2+6,2+7$.

HaRVEY, 2, 3 .

HASSE, I 12,155 .

Head, 145.

Head-fold, 29, 33, 34, 48, 57 .

Heart, 63-66, $122,123,172,173$, $190-196$.

Hen's egg, structure of, I I-I9; changes in, before it is laid, I9-26.

Hensen, 82 .

Hepatic veins, 210.

Hering, I33.

Hind-brain, 78,93 .

His, $16,48,52,55,64,68,7$ i, 72 , $8_{2}, 8_{3}, 1_{2}, I_{34}, 149,153,156$, I59, 168 .

HOPPE-SEILER, I 5 .

HUxlex, $76,90,137,159,179,181$, $182,228$.

Hypoblast, $27,45,51,56,19^{8}$.

Hypophysis cerebri, 9I.

\section{I.}

Iliac arteries, 168,214 . Imbedding, $248-250$.

Incubation, 27-42.
Incubators, 239, 2 +0.

Infundibulum, 91,92 .

Intermediate cell-niass, $S_{2},{ }_{3} 6,161$, 165.

Intermedium, 15,176 .

Investing mass, $177,178,225-227$.

Iter a tertio ad quartum ventriculum, 93 .

J.

JAEGER, 158 .

Jugal, 235.

Jugular vein, 20\%.

K.

KAPFF, 167.

Kidneys, permanent, $16_{3}, 16_{4}$.

Klein, 65,72 .

KleinexberG, $2+6,2+8,2+9$.

Knee, 175 .

KOELLIKER, 6, 71, 82, 101, IOS, т33, I $34,159,18$ I.

KUPFFER, 163 .

I.

Lacrymals, 237.

Laminæ dorsales, 48 .

Lateral column, I86, 187 .

Lateral folds, 76 ; plate, $5+$; latera 1 ventricle, $9 \mathrm{I}$.

Lens, 98, 107,108 .

Lens-capsule, 108, 109.

LEOPOLD, I67.

Lieberkuehs, 98, 101, 102, 107, 108.

Ligamentum suspensorium, 158.

Limbs, $143,1+5,174,175$.

Liquor amnii, $4 \mathrm{I}$.

Liver, I3I-I33.

Lungs, I 29-I 3 I.

Lung-vesicle, primary, $13 \mathrm{I}$.

II.

MIALPIGHT, 3 .

Malpighian bodies, $\mathrm{I} \sigma_{7}$.

Mandibular arch, 230.

Manus, 175,176 .

Marginal process, 233 .

Naxilla, I 20.

Maxillares, 233 .

Maxillary processes, 179, 229 .

Maxillo-palatine, 235.

Meatus venosus, 123, 169, 229. 
MEckel's cartilage, I79, I 82,229 , 230.

Medulla oblongata, 93 .

Medullary canal, 53; folds, $3 \pi, 48$, 57 ; groove, 48,57 .

Nembrana limitans externa, 106.

Membrane of Descemet, 102; of Reissner, II2; vitelline membrane $13,20$.

Membrane-bones, ז $78,233,237,238$.

Membranous labyriuth, II I-I I 3 .

Meniscus, 158 .

Mesenteric veins, 17 I, 2 I0.

Mesentery, 127.

Nesoblast, $27,45,56$, 196, I9S, I99.

Metacarpals, 175,176 .

Metatarsal bones, 76 .

Metatarsus, 176 .

Mid-brain, $78,93,145$.

Miescher, 15 .

Nouth, 179, 182, I 83 .

Movements of the embryo, 223, 224 .

Mucous layer, 45 .

Mueluer, 92, 93, I33, $134,156,157$, 228 ; duct of M. I 44 ; M.'s duct, I 26,162 ; fibres of M., ${ }^{\circ} 7$.

Muscies, episkeletal, I 59 .

Muscle-plates, I 35 , I 36 , I 59.

\section{N.}

Nails, 205 .

Nasal labyrinth, I8I ; pits, II7, I45; processes, iso, 233 .

Nasals, 235 .

Nathusius, II.

Neck, II9.

Nerves; cranial, I37, I38, I47 ; glossopharyngeal, I 47 ; optic, 107 ; pneumogastric, 147 .

Neural canal, 53 ; tube, 37 .

Notochord, 49, $57,78,156,157$.

Nucleus of Pander, 15 ; nucleus pulposus, $15^{8}$.

\section{O.}

Occipital bone, $22 \%$.

Occipital condyle, $230,233,236$.

Occipital foramen, 227.)

OELLACHER, 2O, 2I, 22, 25.

(Esophagus, 12\%.

Olfactory vesicle, I 7 .

Omphalo-mesaraic arteries, 66, 80, $168,202,214$; veins, $63,69,80$, I 70,202 .
Opening the egg, 240, $24 \mathrm{I}$.

Opisthotic, 237.

Optic cup, 97, 103; nerve, 107; vesicles, $76,77,94,95,96$.

Osseous labyrinth, I I I-I I 4 .

Ossification, 206; of the cranium, 233 , 235 ; of the ear, 237.

Otic vesicle, II I.

Ovarian ovum, I9.

Oviduct, 20, I68.

Ovum, descent of the, $2 \mathrm{I}$; primordial ova, 166,167 .

P.

Paired rods of cartilage, 227 .

Palatinè, 233.

Palatine rod, 230, 232.

Pancreas, I33.

PANder, 5, 7I ; nucleus of $P, I_{5}, I_{7}$.

Parasphenoid, 235.

Parietals, 235 .

Parker, r77, 179, 225, 226, 228, 229, 23 I, 232, 234, 236 .

Parostosis, 233.

Parovarium, 167 .

Pecten, 105.

Pellucid area, 57. See Area pellucida.

Peremenchio, 133 .

Pericardium, 194.

Periotic capsules, 227.

Pes, 176 .

Pflueger, 167 .

Phalanges, 176 .

Pineal gland, 9r.

Pituitary body, 9r, 92, 227 ; diverticulum, 92,$93 ;$ space, $I_{7} 8$, $227,232$.

Pleuroperitoneal cavity, 38,54 .

Pnelumogastric nerve, 147 .

Portal vein, 2 Io.

Posterior fissure, 187 .

Post-frontal, 237 .

Premaxillaries, 233, 237.

Prenasal cartilage, 23 I, 232.

Preservation of the embryo as a whole, 252.

Primary lung-vesicle, $\mathbf{I} 3 \mathrm{I}$.

Primitive aortx, 66, 79; groove, 47, 57 ; streak, 46, 47, 56 .

Primordial ova, I66, i67.

Prootic, 23\%.

Protovertebræ, .55, 5Ћ, 134, I5II 59 .

Pterotic, 237. 
Pterygoid rod, 230, 232.

Pterygo-palatine rod, 179 .

Punctum saliens, 2.

Purkinje, 6.

Quadrate, I $79,230,232,236$.

Quadrato-jugals, 235 .

R.

Fiadiale, 175 .

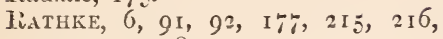
$217,225,228$.

Recessus vestibuli, II I, II 5 .

Reichert, 9i, i 48 .

RiEISSNER, membrane of, I 12.

Rejiak, 6, 64, 71, 72, $78,82,97$, ror, 106, 108, 133, 134, 138, I+7, 148 , $150,153,163$.

Removal of the embryo, 242, $26 \mathrm{I}$.

Retina, ${ }^{0} 0_{5}$.

Ribs, $1=8$.

Rods and cones, Io6; rods of Corti, I 7 ; paired rods of cartilage, 227 .

Romiti, $8,3,167$.

Rosenberg, 175,176 .

Rostrum, 232, $235,236$.

S.

Sac; amniotic $4 \mathrm{r}$; embryonic 35 .

Sacculus hemisphericus, I I 5 .

SicheNk, I33, I37.

Schultze, Max, гоб.

SCHWARCK, I $55,157$.

Sclerotic, 102 .

Sections, $250-252,254,255,258,260$, $26 \mathrm{I}, 262,263,264$.

Segmentation, $22-26,26_{4}, 2 \sigma_{5}$; secondary of vertebral column, I 55 .

Segmentation-cavity, 25 .

Semicircular canals, i I5.

Semilunar valves, I91, 192.

Seminiferous tubules. See Tubuli seminiferi.

Septum nasi, 237; septum of the bulbus arteriosus, 215 ; ventricular septum 172,173 .

SERNoFF, 162,167 .

Serous cavity, 38 ; layer, 45 .

Sexual eminence, 166.

Shell-membrane, I 2.

Sinus rhomboidalis, 60, I90; terminalis, 69 ; venosus, 123 , I 70 .

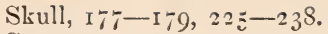

SMIDT, $9 \mathrm{I}$.

Somatic stalk, 39, I 43 .

Somatopleure, $38,54,57$.

Spinal cord, I83-190; ganglia, I52, I53.

Splanchnic stalk, 39, I4I, I 74 .

Splanchnopleure, $38,54,57$.

Spleen, 133.

Splint-bones, 233, 238 .

Splitting of the mesoblast. Sce Cleavage.

Squamosal, 235 .

Staining, $2+7$.

Stapes, 232.

Stomach, I 28 .

STRICKER, I33.

Stroma, I66.

Subclavian arteries, $21 \%$.

Summary of changes during the first day, 56, 57; second day, 83 ; third day, I39, I 40 ; fourth dity, 173; fifth day, 199

Supraoccipital plates, 236 .

Supraoccipitals, 232, 233 .

Surface view of embryo, $2+3,2+4$, $253,256,259,262$.

Sutures, 238 .<smiles>C1=CCC1</smiles>

T.

Tail, I 25,143 .

Tail-fold, 35,75, I $25, \mathrm{I}+3$.

Tarsal bones, 176 .

Tarso-metatarsus, 176 .

Testes, 167 .

Thorson, Allen, 6, I 2.

Thyroid body, I33, I3t.

Tibiale, $1 ; 6$.

TONGE, I9O, I9I, 215 .

Tongue, 205.

Trabeculæ cranii, $178,179,227,228$.

Trachea, I3I.

Tread, 2.

Tubuli seminiferi, 167 .

Tubuli uriniferi, $\mathrm{I} \sigma_{+}$.

'Tween brain, 9 r.

$\mathrm{U}$.

Tinare, I 75 .

Umbilical arteries, I58; duct, I43,

17 ; veins, 172,210 .

Ureter, I63.

Utriculus, I 5 . 


\section{V.}

Tas deferens, 168 .

Vascular area, 28, 56, 57, 84, 85; v. layer, 45 .

Veins; allantoic 210; cardinal 124 ; of the liver, I 7 I; hepatic 2 ro; jugular 207 ; mesenteric $\mathrm{I} 7 \mathrm{I}, 210$; omphalo-mesaraic 63,69 , 8o, 17o, 202 ; portal 210 ; umbilical 172 , 210 ; vena cava inferior, $17 \mathrm{I}, 207$, 209; vena terminalis, 69; venæ advehentes, 171, 209, 2 I0; ven:e cavæ, 209; venæ revehentes, $17 \mathrm{I}$, 209, 210.

Tenous circulation, I $70,2 \mathrm{II}$; venous system, $169-172$.

Tentricles, 79, 193; fourth ventricle 94.

Ventricular septum, $\mathbf{1}_{7} \mathbf{2}, \mathbf{1} 7 \mathbf{3}$.

Tertebral arteries, $2 \mathrm{I} 7$; column, $\mathbf{5}_{\mathbf{5}} \mathbf{2}$ - I55; plate, 54,56 .

Tesicle, germinal, 20 ; olfactory $\mathbf{I}_{7} 7$; vesicle of the cerebral hemispheres, 78 ; optic vesicle $76,77,94,95$, 96 ; otic vesicle in ; vesicle of the third ventricle, $9 \mathrm{I}$.

Testibule, II I.

Visceral arches, 179; clefts, I19, 204; folds, II 9 .
Vis essentialis, 5.

Vitellin, I5.

Vitelline membrane, I3, 20.

Vitreous humour, IOI.

Vomer, 237.

VoN BAER, 5, 6, 7, 41, 64, 65, 7I, $72,78,86,9$ I, 148, I 50, 166, I 73 , I90, 194, 201, 202, 206, 213, 214 , $215,217,219$.

W.

Waldeter, S2, I49, I 60, I 61, I 62 , $163,165,167$.

White of egg, I3.

White columns, I 84, I 85,186, I 89 ; white matter, $184,18 \dot{\xi}, \mathbf{1 8 6}, \mathbf{1} 8{ }_{7}$, I 89 ; white yolk, 15 .

Wing, 175 .

WOLFF, 4, 5, 6, 7I.

Wolffian bodies, I39, I6 $\sigma_{-1} \sigma_{3},{ }^{6} 6_{7}$; Wolffian duct, $73,82,83,138$, I 39 , $160-162,168$; Wolffian ridge, $I_{43}$.

$Y$.

Yolk, I4-ı6, 202; yellow yolk, I4; white yolk, 15 .

Yolk-sac, 35 . 
Bedford Street, Covent Garden, London, Seftember I 874 .

MACMILLAN \& Co.'s CATALOGUE of WORKS in MAthematics and PhystCal SCIENCE; including PURE and APPLIED MATHEmatics; Phystcs, Astronomy, Geclogy, Chemistry, Zoology, Botany; Physiology, ANATOMY, and MEDICAL WORKS generally; and of WORKS in MENTAL and MORAL Philosophy and Allied Subjects.

\section{MATHEMATICS.}

Airy.- Works by Sir G. B. AIry, K.C.B., Astronomer Royal :-

ELEMENTARY TREATISE ON PARTIAL DIFFERENTIAL EQUATIONS. Designed for the Use of Students in the Universities. With Diagrams. New Edition. Crown 8vo. cloth. 5s. $6 d$. It is hoped that the methods of solution here explained, and the int. stances exhibited, will be found sufficient for application to nearly, all the important problems of Physical Science, which require for their complete investigation the aid of Partial Differential Equa. tions.

GN THE ALGEBRAICAL AND NUMERICAL THEORY OF ERRORS OF OBSERTATIONS AND THE COMBINATION OF OBSERVATIONS. Crown 8vo, cloth. $6 s .6 d$. In order to spare astronomers and observers in natural philosophy the confusion and loss of time which are produced by referring to the ordinary treatuses embracing both branches of probabilities (the first relating to chances which can be altered only by the changes of entive units or integral multiples of units in the fundamental conditions. of the problem; the other concerning those chances which have respect to insensible gradations in the value of the element measured), this volume has been drawn up. It relates only to errors of observa. tion, and to the rules, derivable from the consideration of these crrors, for the combination of the results of observations.

UNDULATORY THEORI OF OPTICS. Designed for the Use of Students in the University. New Edition. Crown 8ro, cl. 6s. 6d. The plan of this tract has been to include those fhenomena only which admit of calculation, and the investigations are applied only to phenomena wihich actually have been observed.

UN SOUND AND ATMOSPHERIC VIBRATIONS. With the Mathematical Elements of Music. Designed for the Use of Students of the University. Second Edition, revised and enlarged. Crown 8vo. 9.. 
$\operatorname{Airy}($ G. B.)-continued.

A TREATISE ON MAGNETISM. Designed for the Use of Students in the University. Crown 8vo. 9s. $6 d$.

Ball (R. S., A.M.)-EXPERIMENTAL MECHANICS. A Course of Lectures delivered at the Royal College of Science for Ireland. By Robert Stawell Ball, A. M., Professor of Applied Mathematics and Mechanics in the Royal College of Science for Ireland (Science and Art Department). Royal Svo. I6s.

"IVe have not met with any book of the sort in English. It elucidates instructively the methods of a teacher of the very highest rank. IVe most cordially recommend it to all our readers."-Mechanics' Magazine.

Bayma.-TIIE ELEMENTS OF MOLECULAK MECIIANICS. By Joseph Barma, S.J., Professor of Philosophy, Stonyhurst College. Demy Svo. cloth. Ios. $6 \%$.

Boole.-Works by G. Boole, D.C.L, F.R.S., Professor of Mathematics in the Queen's University, Ireland:-

A TREATISE ON DIFFERENTIAL EQUATIONS. Third Edition. Edited by I. Todhunter. Crown 8vo. cloth. I 4 s.

"A treatise incomparably superior to any other clementary book on the subject with which we are acquainted."-Philosophical Magazine.

A TREATISE ON DIFFERENTIAL EQUATIONS. Supplementary Volume. Edited by I. Todiunter. Crown Svo. cloth. 8s. $6 d$.

THE CALCULUS OF FINITE DIFFERENCES. Crown Svo. cloth. Ios. 6d. New Edition revised.

Cambridge Senate-House Problems and Riders, WITH SOLUTIONS :--

I 848-I851.-PROBLEMS. By Ferrers and JACKson. Svo, cloth. I 5 s. $6 d$.

I848-I851.-RIDERS. By JAMESON. Sro. cloth. 7s. 6 d.

1854.-PROBLEMS AND RIDERS. By WALTON and Mackenzie. 8vo, clotl. ios. $6 \%$.

IS57.-PROBLEMS AND RIDERS. By CAMPION and IVALTON. Svo, cloth. Ss. 6\%.

I86o.-PROBLEMS AND RIDERS. By WATSON and Routr, Crown 8vo. cloth. $7 s .6 d$.

r864. - PROBLEMS AND RIDERS. By WALTON and WILKINSON. Svo. cloth. Ios. 6 .

These volumes will be found of great value to Teachers and Students as indicating the style and range of mathematical study in the University of Cambridge.

Cheyne.-Torks by C. H. H. Cheyne, M.A., F.R.A.S.:-

AN ELEMENTARY TREATISE ON THE PLANETARY TIIEORY. With a Collection of Problems. Second Edition. Crown Svo. cloth. 6s. 6 d. 
Cheyne.-continued.

THE EARTH'S MOTION OF ROTATICA. CrOWn STO. 3s. $6 \%$.

Childe.-THE SINGULAR PROPERTIES OF TI E ELITP. SOID AND ASSOCIATED SURFACES OF THE NTII DEGREE. By the Rev. G. F. CHILde, M.A., Author of "Ray Surfaces," "Related Caustics," \&c. Sro. Ios. $6 d$.

Dodgson.-AN ELEMENTART TPEATISE ON DETERMINANTS, with their Application to Simultaneous Linea Equations and Algebraical Geometry. By Charles L. Dodgsox, M.A., Student and Mathematical Lecturer of Christ Church.t Oxford. Small 4to. cloth. Ios. $6 d$.

Earnshaw (S., M.A.)-PARTIAL DIFFERENTIAI. EQUATIONS. An Essay towards an entirely New Methorl of Integrating them. By S. EARxshaw, M.A., of St. John's College, Cambridge. Crown Svo. 5 s.

"One of the few English books containing original matrematics." Nature.

Ferrers.-AN ELEMENTARY TREATISE ON TRIIINEAR CO-ORDINATES, the Method of Reciprocal Polars, and the Theory of Projectors. By the Rev. N. M. Ferrers, M.A., Fellow and Tutor of Gonville and Caius College, Cambridge. Seconi! Edition. Crown Sro. 6s. 6d.

Frost.-Works by Percival Frost, M.A., late Fellow of St. John's College, Mathematical Lecturer of King's Coll. Cambridge:THE FIRST THREE SECTIONS OF NEIVTON'S PRINCIPIA. With Notes and Illustrations. Also a Collection of Problems, principally intended as Examples of Newton's Methods. Second Edition. Svo. cloth. Ios. $6 d$.

\section{AN ELENENTARY TREATISE ON CURVE TRACINC.} Svo. I2s.

The author has written this book under the conviction that the shill? and power of the young mathematical student, in order 20 be thoroughly available afterwards, ought to be dizicloped in all possible directions. In order to understand the work it is not nccessary to have much knowledge of what is called Higher Algcbra, nor of Algebraical Geometry of a higher kind than that which simply relates to the Conic Sections.

Frost and Wolstenholme.-A TREATISE ON SOLID GEOmetry. By Percival Frost, M.A., and the Rev. J. Wolstenholme, M.A., Fellow and Assistant Tutor of Christ's College. Svo. cloth. ISs. 
Godfray.- Works by IIUgi Godfray, M.A., Mathematical Lecturer at Pembroke College, Cambridge :-

A TREATISE ON ASTRONOMY, for the Use of Colleges and Schools. Svo. cloth. I2s. 6 d.

"It is a working book," says the Guardian, "taking Astronomy in its proper place in the Mathematical Sciences. . . . It is a book which is not likely to be got up unintelligently."

AN ELEMENTARY TREATISE ON THE LUNAR

THEORY, with a Brief Sketch of the Problem up to the time of Newton. Second Edition, revised. Crown 8vo. cloth. 5s. 6d.

"As an elcmentary treatise and introduction to the subject, we think it may justly claim to supersede all former ones."-London, Edinburgh, and Dublin Phil. Magazine.

Green (George).-MATHEMATICAL PAPERS OF THE LATE GEORGE GREEN, Fellow of Gonville and Caius College, Cambridge. Edited by N. M. Ferrers, M.A., Fellow and Tutor of Gonville and Caius College. 8vo. $15 s$.

The publication of this book may be opportune at present, as several of the subjects with which they are directly or indirectly concerned have recently been introduced into the course of mathematicai study at Cambridge. They have also an interest as being the work of an almost entirely self-taught mathematical genius. "It has becn for some time recognized that Gren's writings are amongst the most valuable mathematical productions we possess." Athenæum.

Hemming.-AN ELEMENTARy TREATISE ON THE DIFFERENTIAL AND INTEGRAL CALCULUS. For the Use of Colleges and Schools. By G. W. Hemming, M.A., Fellow of St. John's College, Cambridge. Second Edition, with Corrections and Additions. 8vo. cloth. 9s.

"There as no book in common use from which so clear and exact a knowledge of the principles of the Calculus can be so readily obtained." - Literary Gazette.

Jackson.-GEOMETRICAL CONIC SECTIONS. An Elementary Treatise in which the Conic Sections are defined as the Plane Sections of a Cone, and treated by the Method of Projections. By J. Stu.art Jackson, M.A., late Fellow of Gonville and Caius College. Crown Svo. 4s. $6 d$.

This work has been writtch with a view to give the student the benefit of the Method of Projections as applicd to the Ellipse and Hyperbola.

Kelland and Tait.-AN INTRODUCTION TO QUATERNIONS. With numerous Examples. By P. Kelland, M.A., F.R.S., and P. G. TAIt, M.A., Professors in the department of Mathematics in the University of Edinburgh. Crown Svo. $7 s .6 d$. This work is an attempt to make it possible to introduce the subject of Quatemions into an Elemcntary Course of Mathematics; it is 
written for those who desire to bccome mathematicians. In thi first nine chapters Prof. Kelland endcavours to iilnustrate and enforce the principles of the scicnce; the last chapter, by Profs Tait, is an introduction to the application of Quaternions to the region biyond that of pure geometry.

Morgan.-A COLLECTION OF PROBLEMS AND EXAMPLES IN MATHEMATICS. With Answers. By H. A. Morgan, M.A., Sadlerian and Mathematical Lecturer of Jesus College, Cambridge. Crown Svo. cloth. $6 s, 6 i$.

Newton's Principia.-4to. cloth. 31s. $6 d$.

It is a sufficient guarantee of the reliability of this complete edition of Newton's Principia that it has been printed for and under the care of Professor Sir William Thomison and Professor Blackburn, of Glasgow University.

Parkinson.-A TREATISE ON OPTICS. By S. PARKINson, D.D., F.R.S., Fellow and Tutor of St. John's College, Cambridge. Third Edition, revised and enlarged. Crown Svo. cloth. IOs. $6 d$.

Phear.-ELEMENTARY HYDROSTATICS. With Numerous Examples. By J. B. PheAr, M. A., Fellow and late Assistant Tutor of Clare Coll. Cambridge. Fourth Edition. Cr. Svo. cloth. 5s. 6d.

Pratt.-A TREATISE ON ATTRACTIONS, LAPLACE'S FUNCTIONS, AND THE FIGURE OF THE EARTH. By John H. Pratt, M.A., Archdeacon of Calcutta, Author of "The Mathematical Principles of Mechanical Philosophy." Fourth Edition. Crown Svo. cloth. $6 s .6 d$.

Routh.-AN ELEMENTARY TREATISE ON THE DYNAMICS OF THE SYSTEM OF RIGID BODIES. With numerous Examples. By Edivard John Routh, M.A., late Fellow and Assistant Tutor of St. Peter's College, Cambridge ; Examiner in the University of London. Second Edition, enlarged. Crown 8vo. cloth. I4s.

Tait and Steele.-DYNAMICS OF A PARTICLE. With numerous Examples. By Professor Tait and Mr. Steele. New Edition. Crown 8vo, cloth. IOs. $6 d$.

Thomson.-PAPERS ON ELECTROSTATICS AND MAGNETISM. By Professor Sir IVIlliam Thomson, F.R.S. Svo. ISs.

"In the whole range of modern mental activity and rese scch, there is perhaps nowhere to be found any such amount of purcly scicntific matter, free from all speculation whatever, as is to be found in these diversified and masterly papers on the nearly allied subjects of elcctricity and magnetism. There is scarcaly a paper in all the forty-two in which there is not something interesting, wirition in a clear, unambiguous, and manly style."-Scotsman. 
Todhunter.-IVorks bj I. Todhunter, M.A., F.R.S., of St. Toh 's College, Cambridge :-

"Mi. Thathunter is chicfly known to stu" of mathenatics as the autior of a serics of admirable mitinematical text-books, which possess the rare qualities of being clexr in syle and absolutcly free from mistakes, typograptical or other."--Soturday Review.

A TREATISE ON SPHERICAL TRIGO NOMETRY. Third Edition, enlarged. Crown 8vo. cloth. 4s. $6 d$.

PLANE CO-ORDINATE GEOMETRY, as applied to the Straight Line and the Conic Sections. With numerous Examples. Fifth Edition. Crown 8vo. cloth. $7 s .6 d$.

$A$ TREATISE ON THE DIFFERENTIAL CALCULUS. With numerous Examples. Sixth Edition. Crown 8vo. cloth. IOS. 6 d.

A TREATISE ON THE INTEGR.AL CALCULUS AND ITS APPLICATIONS. With numerous Examples. Fourth Edition, revised and enlarged. Crown Svo. cloth. Ios. 6d.

EXAMPLES OF ANALYTICAL GEOMETRY OF THREE JIMENSIONS. Third Edition, revised. Crown 8vo. cloth. 4s.

A TREATISE ON ANALYTICAL STATICS. With numerous Exansle.s. Thind Edition, revis $i \mathrm{i}$ and enlarged. Crown Sro. cloth. Ias. $6 \%$

A HITORY OF THE IITHEMATICAL TIEORY OF PR.OBABILITI, from the Time of Pascal to that of Laplace. Svo. IS.

RESEARCHES IN TIE CALCULUS OF VARIATIONS, Principally on the Theory of Discontinuous Solution:s: An Essay 1. which the f.dams' Prize was awarded in the University of Cambriage in $187 \div$. Svo. $6 s$.

A HISTORY OF THE MATHEMATICAL THEORIES OF ATTRACTION, and the Figure of the Earth, from the time of Newton to that of Laplace. "Two vols. 8 vo. 24 s.

"Such historics as tiis are at fresent more valuable than original wark. They' at once cnatie the wathematician to make himself ") ister of all that has been done on the subject, and also give in: a che to the right meilod of dealing with the subject in fil'er, by showing him the patis by which advance has ben mat in the past. . . . It is with ammingled satisfaction tivat we see this work adopied as his special subject by one whos: cast of mind and self-culture have made him one of the $3:$ accurate, as he certainly is the most leamed, of Cambri "re mathematicians."-Saturday Review. "Probably no man in England is so qualified to do justice to the theme as Mr. Iodhenter. To all mathomaticians "se volumes will be detply interesiing, and to all succeeding investigators, of the highest practical ritiity." - $\Lambda$ thenecum. 
Wilson ( $\bar{V}$. P.) - A TREATISE ON DYNAMICS. By W. P. IVILson, M.A., Fellow of St. John's College, Cambridge, and Professor of Mathematics in Queen's College, Belfast. Svo. 9s. $6 a$.

Wolstenholme.-A BOOK OF MATHEMATICAL PROBLEMS, on Subjects included in the Cambridge Course. By Joseph Wolstenholme, Fellow of Christ's College, some time Fellow of St. John's College, and lately Lecturer in Mathematics at Christ's College. Crown 8vo. cloth. 8s. $6 d$.

Young.-SIMPLE PRACTICAL METHODS OF CALCU LATING STRAINS ON GIRDERS, ARCHES, AND TRUSSES. With a Supplementary Essay on Economy in suspension Bridges. By E. W. YousG, Associate of King's College, London, and Member of the Institution of Civil Engineers. Svo. $7 s .6 d$.

"An cxcellent combination of theoretical methods of finding strains in beams and structures, as modified by practical experience. The reasoning is clear, and the equations are simple enough, and do not require more than a knowledge of elementary algebra and trigonometry for their solution. The diagrams are especially clear." Architect.

\section{PHYSICAL SCIENCE.}

Airy (G. B.)-POPULAR ASTRONOMY. With Illustrations. By Sir G. B. AIry, K.C.B., Astronomer Royal. Seventh and cheaper Edition. ISmo. cloth. $4 s .6 d$.

Rastian.-THE BEGINNINGS OF LIFE: Being some Account of the Nature, Modes of Origin, and Transformations of Lower Organisms. By H. Charlton Bastian, M.D., F.R.S., Pro fessor of Pathological Anatomy in University College, London, \&c. In Two Volumes. With upwards of 100 Illustrations, Crown Svo. 28 s.

"It is a book that cannot be ignored, and must ineritably lead to renewed discussions and repeated observations, and through these to the establishment of truth."-A. R. WALLACE in Nature.

\section{Blanford (W. T.)-GEOLOGY AND ZOOLOGY OF}

ABISSINIA. By WV. T. Blaxford. Svo. $2 \mathrm{I}$ s.

This work contains an account of the Geological and Zoological Observations made by the author in Abyssinia, when accompanying the British Army on its march to Magdala and back in 186S, and during a short journey in Northem Abyssiniz, after the departure of the troops. Part I. Personal Narrative; Part II. Geology"; Part III. Zoology. IVith Coloured Illustrations and Geological Map. "The result of his labours," the Academy say's, "is an important contribution to the natural history of the country." 
Clodd.-THE CHILDHOOD OF THE WORLD: a Simple Account of Man in Early Times. By Edward Clod, F. R.A.S. Third Edition. Globe Svo. 3s. Special Edition for Schools. I 8 mo. I $s$.

"Likely, we think, to prove acceptable to a large and growing class of readers."-Pall Mall Gazette.

Professor MAX MUller, in a ietter to the author, says: "I read your book with great pleasure. I have no doubt it will a'o good, and Thope you will contimue your work. Nothing spoils our tempen so much as having to mulearn in youth, manhood, and covn ola age, so many things which we were taught as children. A book like yours will prepare a far better soil in the child's mind, and 1 was delighted to have it to read to my children."

Cooke (Josiah P., Jun.)-FIRST PRINCIPLES OF CHEMiCAL PHILOSOPHY. By Josiah P. Cooke, Jun., Ervine Professor of Chemistry and Mineralogy in Harvard College. Third Edition, revised and corrected. Crown Svo. I2s.

The object of the author in this book is to present the philosophy of Chemistry in such a form that it can be made with profit the subject of College recitations, and furnish the teacher with the means of testing the student's faithfulness and ability. With this view the snbject has been developed in a logical order, and the principles of the science are taught indepenuently of the experimental evidence on which they rest.

Cooke (M. C.)-HANDBOOK OF BRITISH FUNGI, with full descriptions of all the Species, and Illustrations of the Genera. By M. C. Cooke, M.A. Two vols. crown Svo. 245. "No effort has been spared to make the work worthy of confidence, and, by the publication of an occasional supplement, it is hoped to maintain it for many years as the "Handbook" for every student of British Fungi. Appended is a complete alphabetical Index of all the divisions and subdivisions of the Fungi noticed in the text. The book contains 400 figures. "Will maintain its place as the standard English book, on the subject of which it treats, for many years to come." - Standard.

Dawkins, - CAVE-HUNTING : Researches on the Evidence of Caves respecting the Early Inhabitants of Europe. By W. Boy Dawkins, F.R.S. Illustrated. Svo.

Dawson (J. W.)-ACADIAN GEOLOGY. The Gcologic Structure, Organic Remains, and Mineral Resources of Nova Scotia, New Brunswick, and Prince Edward Island. By JohN William Dawson, M.A., LL.D., F.R.S., F.G.S., Principal and Vice-Chancellor of M'Gill College and University, Montreal, \&.c. Second Edition, revised and enlarged. With a Geological Map and numerous Illustrations. Svo. I $S$ s.

"The book will doubtless find a place in the library, not only of the sciontific geologist, but also of all who are desirous of the in- 
dustrial progress and commercial prosperity of the Acadian provinces." - Mining Journal. "A style at once popular and scientific. .. A valuable adatition to our store of geological knoziledge." Guardian.

Galton.-Works by Francis Galion, F.R.S. :-

METEOROGRAPHICA, or Methods of Mapping the Weather. Illustrated by upwards of 600 Printed Lithographic Diagrams. 4to. 9 s.

"If the various Governments and scientific bodies would perform for the whole world for two or three years what, at a great cost and labour, Mr. Galton has done for a fart of Europe for one month, Meteorology would soon cease to be made a joke of."-Spectator.

HEREDITARY GENIUS: An Inquiry into its Laws and Consequences. Demy 8vo. I2s.

"I propose," the anthor says, "to show in this book that a man's natural abilities are derived by inheritance, under exactly the same limitations as are the form and fhysical fiatures of the whole organic world. The Times calls it "a most able and most interesting book;" and Mr. Darwin, in his "Descent of Mcm" (vol. i. p. I I I), says, "WVe know, through the admirable labours of MIr. Galion, that Genius tends to be inherited."

Geikie (A.)-SCENERY OF SCOTLAND, Viewed in Connection with its Physical Geography. With Illustrations and a new Geological Map. By Archibald Geikie, Professor of Geology in the University of Edinburgh. Crown Svo. IOs. $6 d$.

"We can confidently recommend. Mr. Gikit's work to those who wish to look below the surface and read the physical history of the Scencry of Scotland by the light of modern science."-Saturday Review. "Amusing, picturesque, and instructive."-Times.

Guillemin.-THE FORCES OF NATURE : A Popular Introduction to the Study of Physical Phenomena. By Amédée Guillemin. Translated from the French by Mrs. Norman Lockyer ; and Edited, with Additions and Notes, by J. Norman LOCKYER, F.R.S. Illustrated by II Coloured Plates and 455 Woodcuts. Second Edition. Imperial 8vo. cloth, extra gilt. 3 Is. 6 d.

The present work consists of Seven Books, each divided into a number of Chapters, the Books treating respectively of Grazity, Sound, Light, Heat, MIagnetism, Electricity, and Atmospheric Meteors. "Translator and Editor have done justice to their trust. The text has all the force and flow of orisinal writing, combining faithfulness to the author's meaning with purity and independence in regard to idiom; while the historical pricision and accuracy pera'ading the work throughout, spath of the ivatch ful editorial sutpervision which has been given to every scientific detail. Nothing can well exceed the clearness and delicacy of the illustrative woodiuts, borrowed from the Frinch edition, or the 
purity and chromatic truth of the coloured plates. Altogether, the work may be said to have no parallel, either in point of fulness or attraction, as a popular manual of physical science.

What we feel, however, bound to say, and what we say with pleasure, is, that among works of its class no publication can stand comparison cither in literary completencss or in artistic grace with it. "-Saturday Review.

\section{Henslow.-THE THEORY OF EVOLUTION OF LIVING} TIIINGS, and Application of the Principles of Evolution to Religion considered as Illustrative of the WVisdom and Beneficence of the Almighty. By the Rev. George Henslow, M.A., F.L.S. Crown Svo. $6 s$.

"The author is highly philosophical, profound, and accurate in arguments. . . His literary merits are of the highest order. . . . He has certainly written on the whole with much force, brevity, and to the point." - Morning Post. "Several previonsiy accepted axioms of Nitural Theology are showon to be incompatible with the existing position of biological science, and their weakness is well brought forward. . . . In one thing IIr. Henslow has done great good: he has shown that it is consistint with a full dogmatic belief to hold opinions very different from those taught as Natural Theology :ome half-century ago." - Nature.

\section{Hooker (Dr.)-THE STUDENT'S FLORA OF THE} BRITISH ISLANDS. By J. D. HookER, C.B., F.R.S., M.D., D.C.L., Iresident of the Royal Society. Globe Svo. IOs. 6 .

The object of tinis work is to supply students and field-botanists with a fuller account of the Plants of the Eiritish Islands than the manuals hitherto in use aim at giving. "Cortainly the fullest and most accurate manual of the lizid that has yet appeared. Dr. Hooker has shown his characteristic industry and ability in the care and shill which he has throwin into the characters of the plants. These are to a great extent original, and are really admirable for thir combination of clearness, brevity, and completeness." - I'all Mall Gazette.

\section{Huxley (Professor).-LAY SERMIONS, ADDRESSES,} ANi) REVIEIVS. By T. H. Huxley, IL.D., F.R.S. New and Cheaper Edition. Crown 8vo. 7s. $6 \mathrm{cl}$.

Fourten Discourses on the following suljects:--(1) On the Adrisableness of Improving Natural is nowledge:-(2) Emancipationlilack and White:-(3) A Liberal Education, and where to find it:-(4) ScientificEducation:-(5) On the Educational Value of the Natural Ilistory Sciences:-(6) On the Study of Ziology:(7) On the Physical Basis of Life:-18) The Scientific Aspects of Positivism:-(9) On a l'icce of Chril:-(ro) Giological Contemporaneity and I'ersistent J'jizis of Life:-(I I) Giological Reform:(12) The Origin of Spicis:-(13) Criticisms on the "Origin of 


\section{Huxley (Professor)-continued.}

Species:"-(I4) On Descartes" "Discourse touching the Method of using One's Reason rightly and of seeking Scicntific Truth."

CRITIQUES AND ADDRESSES. Svo. Ios. $6 d$.

These "Critiques and Addresses," like the "Lay Sermons," \& c., pub. lished three years ago, deal chiefly with educational, scientific, and philosotlicical sibbjects; and, in fact, as the author says, "indicate the high-water mark of the varions tides of occupation by authich 1 haz' been carried along since the beginning of the year I870." The following is the list of Contents:-I. Administrative Nilitism. 2. The School Boards: what thsy can do, and what they may an. 3. On Medical Education. 4. Yast. 5. On the Formation of Coal. 6. On Coral and Coral Reefs. 7. On the Method's and Results of Ethnology. 8. On sone Fixed Points in British Ethnolosy. 9. Palaontology and the Doctrine of Evolution. 10. Biogenesis and Abiogenesis. Ir. Mr. Darwin's Critirs. I2. The Gencuiogy of. Animals. 13. Bishop Berkcley on the Metaphysics of Senscition.

LESSONS IN ELEMENTARY PHYSIOLOGY. With numerous Illustrations. New Edition. ISmo. cloth. $4 s .6 a^{\prime}$.

This book describes and explains, in a series of graduated lessons, the principles of IHuman Physiology, or the Structure and Functions of the Human Body. "Pure gold throughout."-Guardian. "Unquestionably the clearest and most complete elementarv treatise on this subject that we possess in any language."-." estminster Review.

Jellet (John H., B.D.) - A TREATISE ON THE THEORY OF FRICTION. By JUHN H. JELLET, B.D., Senior Fellow of Trinity College, Dublin ; President of the Royal Irish Academy. Svo. 8s. 6 d.

"The book supplies a "i" nt rohich has hitherto existed in the science of pure mechanics." - Enrineer.

Jerons - THE PRINCI ES OF SCIENCE. A Treatise on iogic and Scientific Method. By W. Stanley Jevors, F.R.S., Proiessor of Logic and Political Economy at Owens College, Manchester. 2 vols. Svo. 25 s.

"We believe that this will be recognized in the future as one of the most valuable pirilosofhical works of our time." -Nanchester Examiner.

Jones.-THE OWENS COLLEGE JUNIOR COURSE CF PRACTICAL CHEMISTRY. By FraNCIS Jones, Chemical Master in the Grammar School, Manchester. With Freface by Professor Roscoe. New Edition. I8mo. with Illustrations. 2s. $6 d$.

Kingsley.-GLAUCUS: OR, THE WONDERS OF THE SHORE. By Charles Kingsley, Canon of Westminster. New Edition, revised and corrected, with numerous Coloured Plates. Crown Svo. 5s. 
Kirchhoff (G.)-RESEARCHES ON THE SOLAR SPEC. TRUM, and the Spectra of the Chemical Elements. By G. Kirchinoff, Professor of Physics in the University of Heidelberg. Second Part. Translated, with the Author's Sanction, from the Transactions of the Berlin Academy for IS62, by IENRY R. Roscoe, B.A., Ph.D., F.R.S., Professor of Chemistry in Owens College, Manchester. Part II. 4to. $5^{s}$.

Lockyer (J. N.)-Works by J. Norman Lockyer, F.R.S.ELEMENTARY LESSONS IN ASTRONOMY. With numerous Illustrations. New Edition. I8mo. 5 s. 6 l.

"The book is full, clear, sound, and worthy of attention, not only as a popular exposition, but as a scientific 'Index.' "- Athenæum. "The most fascinating of elementary books on the Sciences." Nonconformist.

THE SPECTROSCOPE AND ITS APPLICATIONS. By J. Nornian Lockyer, F.R.S. With Coloured Plate and numerous Illustrations. Second Edition. Crown Svo. $3 s .6 d$.

This forms Volume One of "Nature Series," a series of popular Scientific Works now in course of publication, consisting of popular and instructive works, on particular scientific subjects-Scientific Discovery', Applications, History, Biography-by some of the most eminent scientific men of the day. They will be so zuritten as to be interesting and intelligible even to non-scientific readers.

CONTRIBUTIONS TO SOLAR PHYSICS. By J. Norman Lockyer, F.R.S. I. A Popular Account of Inquiries into the Physical Constitution of the Sun, with especial reference to Recent Spectroscopic Researches. II. Communications to the Royal Society of London and the French Academy of Sciences, with Notes. Illustrated by 7 Coloured Lithographic Plates and 175 Woodcuts. Royal 8vo. cloth, extra gilt, price 3is. $6 d$.

"The first part of the work, presenting the reader with a continuous sketch of the history of the various inquiries into the physical constitution of the sun, cannot fail to be of interest to all who care for the revelations of modern science; and the interest will be enhancid by the excellence of the numerous illustrations by which it is accompanied."-A Ahenæum. "The book may be taken as an authentic exposition of the present state of science in connection with the $i m$. portant subject of spectroscopic analysis. . . . Even the unscientific public may' derive much information from it."-Daily News.

Lubbock.-THE ORIGIN AND METAMORPHOSES OF INSECTS. By Sir JoHn Lubbock, M.P., F.R.S. With Numerous Illustrations. Second Edition. Crown Svo. 3 s. $6 d$. This volume is the second of "Nature Series." The Athenæum says: "It is written in a clear and pleasing style, like all the author's scientific treatises, and is niccly illustrated with outline wood-cuts. We can most cordially recommend it to all young naturalists." "As a summary of the phenomena of insect meta- 
morphoses his little book is of great value, and will be read with interest and profit by all students of natural history. The whole chapter on the origin of insccts is most interesting and valuable. The illustrations are numerous and good." - Westminster Review.

Macmillan (Rev. Hugh).-For other Works by the same Author, see Theological Catalogue.

HOLIDAYS ON HIGH LANDS; or, Rambles and Incidents in search of Alpine Plants. Globe 8vo. cloth. $6 s$.

The aim of this book is to impart a general idea of the origin, character, and distribution of those rare and beautiful Alpine plants which occur on the British hills, and which are found almost everywhere on the lofty montain chains of Enrope, Asia, Ajrica, and America. The information the author has to give is conveyed in a setting of personal adventure. "One of the most charming books of its kind ever written."-Literary Churchman. "Mr. MI's glowing pictures of Scandinavian scenery." - Saturday Review.

FIRST FORMS OF VEGETATION. Second Edition, corrected and enlarged, with Coloured Frontispiece and numerous Illustrations. Globe 8vo. $6 s$.

The first edition of this book was published under the name of "Footnotes from the Page of Nature; or, First Forms of Vigetation." This edition contains upwards of 100 pages of nean matter and eleven new illustraíions. "Probably the best popular guile to the study of mosses, lichens, and fungi ever written. Its practical value as a help to the student and collector cannot be exagoerated." -Manchester Examiner.

Mansfield (C. B.) - A THEORY OF SALTS. A Treatise on the Constitution of Bipolar (two-membered) Chemical Compounds. By the late Charles Blachford Massfield. Crown 8vo. I4s.

Miller.-THE ROMANCE OF ASTRONOMY. By R. KALlEy Miller, M.A., Fellow and Assistant Tutor of St. Peter's Col lege, Cambridge. Crown Svo. 3 s. $6 d$.

"On the whole, the information contained is of a trustworthy character, and we cordially recommend it to the perusal of those who, without being in possession of the knowledge requisite for discussing astronomical theories, or the means by which they are arrived at, are y'at desirous of becoming acquainted with some of the most interesting of astronomical conclusions." - Athenæum.

Mivart (St. George).-Works by St. George Mivart, F.R.S. Sc., Lecturer in Comparative Anatomy at St. Mary's Hospital: ON THE GENESIS OF SPECIES. Crown Svo. Second Edition, to which notes have been added in reference and reply to Darwin's "Descent of Man." With numerous Illustrations. pp. xv. 296. 9 s.

Canon Kingsley, in his address to the "Devonshire Association," says, "Let me recommend carnestly to y'ou, as a specimen of what 
Mivart (St. George)-continued.

can be said on the other sine, the 'Genesis of Species,' by MIr. St. George Mivart, F.R.S. a book which I am happy to say has been received elsewhere as it has deserved, and, I trust, will be reccired so among you." "Yn no work in the Enstish language has this great controversy been treated at once with the same broad and vigorous grast of facts, and the same liberal and candid temper." - Saturday Review.

LESSONS IN ELEMENTARY ANATOMY. With upwards of 400 Illustrations. ISmo. $6 s .6 d$.

"It may be questioned whether any other work on anatomy contains in like compass so proportionately great a mass of information." -Lancet. "We recommend it as one of the most waluable yet published in Messrs. Macmillan's Scientific Series." - Pall Mall Gazette.

Murphy.- Works by Joseph John MURPhy :-

HABIT AND INTELLIGENCE, in Connection with the Laws of Matter and Force: A Series of trientific Essays. Two Vols. Svo. I6s.

"We are pleased to listen," says the Saturday Review, "to a writer who has so firm a foothold upon the ground within the scope of his immediate survey, and who can enunciate with so mich clearness and forcepropositions which come within his grasp."

THE SCIENTIFIC BASES OF FAITH. Svo. I4s.

Nature.-A WEEKLY ILLUSTRATED JOURNAL OF SCIENCE. Published every Thursday. Price $4 \%$. Monthly Parts, I s. 4 $d$. and is. 8 $d$. ; Half-yearly Volumes, Ios. $6 d$. Cases for binding Vols. is. $6 d$.

"Backed by many of the best names among English philusophers, and by a ferv equally valuable supporters in America and on the Continent of Ezrope." -Saturday Review. "This able and well-edited Fournal, which posts up the scicnce of the day promptly', and promises to be of signal service to students and savants. . . . . . Scarcely any expressions that we can employ would exaggerate our sense of the moral and theological value of the work." -British Quarterly Review.

Oliver.-Works by Daniel Oliver, F.R.S., F.L.S., Professor of Botany in University College, London, and Keeper of the Herbarium and Library of the Royal Gardens, Kew :-

LESSONS IN ELEMENTARY BOTANY. With nearly Two Hundred Illustrations. New Edition. I8mo cloth. $4 s .6 d$. This book is designed to teach the elements of Botany on Professor Henslow's plan of selected Types and by the use of Schedules. The earlier chapters, embracing the elements of Structural and Physiological Botany, introduce us to the methodical study of the Ordinol Types. The conchuding chapters are cntitled, "How to Dry 


\section{Oliver.-continued.}

Plants" and "IHow to Discribe Plants." I valuable Glossary is appended to the a'olume. In the preparation of this work free use has bien made of the manuscript materials of the late P'rofessor IIcnslow.

FIRST BOOK OF INDIAN BOTANY. With numerous Illustrations. Extra fcap. Svo. 6s. 6 .

This mannal is, in substance, the author's "Lessons in Elementary Botany," adapted for use in India. In preparing it he has had in vinus the want, often felt, of some handy résumé of Indian Botany, which might be serviceable not only to residents of India, but also to anyone about to proceed thither, desirons of getting some preliminary itica of the botany of the country. It contains a welldigested summary of all essential knowledge pertaining to Indian Botany, wronght out in accordance with the best principles of scientific arrangement."-Allen's Indian Mail.

Penrose (F. C.) - ON A METHOD OF PREDICTING BY GRAPIICAL CONSTRUCTION, OCCULTATIONS OF STARS BY THE MOON, AND SOLAR ECLIPSES FOR ANY GIVEN PLACE. Together with more rigorous methods for the Accurate Calculation of Longitude. By F. C. PExrose, F.R.A.S. With Charts, Tables, \&c. 4to. I2s.

Perry.-AN ELEMENTARY TREATISE ON STEAM. By John Perry, B.E., Whitworth Scholar, \&c., late Lecturer in Physics at Clifton College. With numerous Woodcuts, Numerical Examples, and Exercises. I 8mo. 4s. $6 d$.

"Mr. Perry has this compact little volume brought together an immense amount of information, new told, regarding stiam and its application, not the least of its merits being that it is suited to the capacities alike of the tyro in engincering science or the batter grade of artisan."-Iron.

Pickering.-ELEMENTS OF PHYSICAL MANIPULATION. By E. C. Pickering, Thayer Professor of Physics in the Massachusetts Institute of Technology. Part I., medium Svo. ros. $6 d$. "IVe shall look with interest for the appearance of the second volume, and when finished 'Physical Mranipulation' will no donbt be considered the best and most complete text-book on the subject of which it treats." -Nature.

Rendu.-THE THEORY OF THE GLACIERS OF SAVOY. By M. le Chanolne Rendu. Translated by A. Wells, Q.C., late President of the Alpine?Club. To which are added, the Original Memoir and Supplementary Articles by Professors TAIT and RUSKIN. Edited with Introductory remarks by GEORGE ForBES, B.A., Professor of Natural Philosophy in the Andersonian University, Glasgow. Svo., 7s. 6d. 
Rodwell.-THE BIRTH OF CHEMISTRY. By G. F. Ron. weLL, F.R.A.S., F.C.S. With numerous Illustrations, Crown Sro. 3 s. $6 d$.

"Mr. Rodruell has produced a thoughtful, sugsestive, and decidedly readable book."-Quarterly Journal of Science.

Roscoe.-Works by Henky E. Roscoe, F.R.S., Professor of Chemistry in Owens College, Manchester :-

LESSONS IN ELEMENTARY CHEMISTRY, INORGANIC AND ORGANIC. With numerous Illustrations and Chromolitho of the Solar Spectrum, and of the Alkalies and Alkaline Earths. New Edition. I8mo. cloth. 4s. $6 \mathrm{~d}$.

"IVe unhesitatingly pronounce it the best of all our elementary treatises on Chemistry."--Medical Times.

SPECTRUM ANALYSIS. Six Lectures, with Appendices, Engravings, Maps, and Chromolithographs. Royal 8vo. $2 \mathrm{r} s$.

A Third Edition of these popular Lectures, containing all the most recent discoveries and several additional illustrations. "In six lectures he has given the history of the discovery and set forth the facts relating to the analysis of light in such a way that any reader of ordinary intelligence and information will be able to understand what 'Spectrum Analy'sis' is, and what are its claims to rank among the inost signal triumphs of scicnce." -Nonconformist. "The lectures themselves furnish a most admirable elementary treatise on the subject, whilst by the insertion in appendices to each lecture of exiracts from the most important published memoirs, the author has rendered it equally valuable as a text-book for advanced students."-Westminster Review.

Schorlemmer.-A MANUAL OF THE CHEMISTRY OF THE CARBON COMPOUNDS OR ORGANIC CHEMISTRY. By C. Schorlemmer, F.R.S., Lecturer in Organic Chemistry-in Owens College, Manchester. Svo. I 4 s.

"It appears to us to be as complcte a mamual of the metamorphoses of carbon as could be at present produced, and it must prove eminently useful to the chemical student." - Atheneum.

\section{Stewart (B.)-LESSONS IN ELEMENTARY PHYSICS.} By Balfour Stewart, F.R.S., I'rofessor of Natural Philosophy in Owens College, Manchester. With numerous Illustrations and Chromolithos of the Spectra of the Sun, Stars, and Nebula. New Edition. r8mo. 4s. $6 d$. A descriftion, in an clementary manner, of the most inportant of those laws which regulate the phenomena of nature. The active agents, heat, light, electricity, etc., are regarded as varieties of energy, and the work is so arranged that thir relation to one another, looked at in this light, and the faramount importance of the law's of encrgy, are cliarly, brought out. The volume contains all the necessary illustrations. The Edutcaional Times calls this 
"the beau-ideal of a scientific text-book, clear, accurate, and thorough."

Taylor.-SOUND AND MUSIC : A Non-Mathematical Treatise on the Physical Constitution of Musical Sounds and Harmony, including the Chief Acoustical Discoveries of Professor Helmholtz. By Sedley Taylor, M.A., late Fellow of Trinity Colledge, Cambridge. Large crown 8vo. 8s. $6 d$.

"In no previous scientific treatise do we remember so exhaustive and so richly illustrated a description of forms of vibration and of wave-motion in fluids."-Musical Standard.

Thomson.-THE DEPTIIS OF THE SEA : An Account of the General Results of the Dredging Cruises of H.M.SS. "Porcupine" and "Lightning" lluring the Summers of 1868.69 and 70 , under the scientific direction of Dr. Carpenter, F.R.S., J. Gwyn Jeffreys, F.R.S., and Dr. Wyville Thomson, F.R.S. By Dr. IVyville Thomson, Director of the Scientific Staff of the "Challenger" Expedition. With nearly Ioo Illustrations and 8 coloured Maps and Plans. Second Edition. Royal 8vo. cloth, gilt. 3Is. $6 d$. It was the important and interesting results recorded in this volume that induced the Government to send out the great Expedition now launched under the scientific guidance of Dr. Wyrille Thomson. The Athenæum say's: "Professor Thomson's book is full of interesting matter, and is written by a master of the art of popular exposition. It is excellently illustrated, both coloured maps and woodcuts possessing high merit. Those who have already become interested in dredging operations will of course make a point of reading this work; those who wish to be pleasantly introduced to the subject, and rightly to appreciate the news which arrives froin time to time trom the 'Challenger,' should not fail to scek instruction from Professor Thomson."

Thornton.-OLD-FASHIONED ETHICS, AND COMMONSENSE METAPHYSICS, with some of their Applications. By William Thomas Thornton, Author of "A Treatise on Labour." 8vo. IOs. $6 d$.

The present volume deals with problems which are agitating the minds of all thoughtful men. The following are the Contents:I. Ante-Utilitarianism. II. History's Scientific Pretensions. III. David Hume as a Metaphysician. IV. Huxleyism. V. Recent $P$ ase of Scicntific Atheism. VI. Limits of Demonstrable Theism.

Thudichum and Dupré.-A TREATISE ON THE ORIGIN, NATURE, AND VARIETIES OF WINE. Being a Complete Manual of Viticulture and CEnology. By J. L. WV. Thudichum, M.D., and August Dupré, Ph.D., Lecturer on Chemistry at Westminster Hospital. Medium 8vo. cloth gilt. 25 . "A treatise almost unique for its usefulness either to the winc-grower, the vendor, or the consumer of wine. The analyses of wine are the most complete we have yet sen, exhibiting at a glance the 
constituent principles of nearly all the wines known in this country." - Wine Trade Review.

Wallace (A. R.)-CONTRIBUTIONS TO THE THEORY OF NATURAL SELECTION. A Series of Essays. By, Alfred RUSSEl WAllace, Author of "The Malay Archipelago," etc. Second Edition, with Corrections and Additions. Crown 8vo. 8s. $6 d$. (For other TVorks by the same Author, see CATALOGUE OF HISTORY AND TRAVELS.)

Mr. Wallace has good claims to be considered as an independent originator of the theory of natural selection. Dr. Hooker, in his address to the British Association, spoke thus of the author: "Of Mr. Wallace and his many contributions to philosophical biology it is not easy to speak without enthusiasm; for, putting aside their great merits, he, throughout his writings, with a modesty as rare as I believe it to be unconscious, forgets his owen unquestioned claim to the honour of having originated independently of Mr. Darvin, the theories which he so ably defends." The Saturday Review says: "He has combined an abundance of resh and original facts with a liveliness and sagacity of reasoning which are not often displayed so effectively on so small a scale."

Warington.-THE WEEK OF CREATION; OR, THE ${ }^{\circ}$ COSMOGONY OF GENESIS CONSIDERED IN ITS RELATION TO MODERN SCIENCE. By GEORGE WARInGTON, Author of "The Historic Character of the Pentateuch Vindicated." Crown Svo. 4s. $6 d$.

"A very able vindication of the Mosaic Cosmogony, by a writer who unites the adrantages of a critical knowiledge of the Hebrew text and of distinguished scientific attainments." - Spectator.

Wilson.-Works by the late GEORGE WILson, M.D., F.R.S.E., Regius Professor of Technology in the University of Edinburgh :-

RELIGIO CIIEMICI. With a Vignette beautifully engraved after a design by Sir Noel Paton. Crown 8 vo. 8s. 6 .

"A more fascinating volume," the Spectator says, "has seldom fallen into our hands."

THE PROGRESS OF THE TELEGRAPH. Fcap. 8vo. Is. "While a complete viens of the progress of the greatest of human inventions is obtained, all its suggestions are brought out with a rare thoughtfulness, a genial humour, and an exceeding beauty of utterance."-Nonconformist.

Wilson (Daniel.)-CALIBAN : THE MISSING LINK. By DANIEL Wilson, LL. D., Professor of History and English Literature in University College, Toronto. 8vo. IOs. 6 .

"The whole volume is most rich in the eloquence of thought and imagination as well as of words. It is a choice contribution at once to science, thcology", religion, and literature."-British Quarterly Review. 
Winslow.-FORCE AND NATURE : ATTRACTION AND REPULSION. The Radical Principles of Energy graphically discussed in their Relations to Physical and Morphological Development. By C. F. Winslow, M.D. 8vo. I4s.

"Deserees thoughtful and conscientious study."-Saturday Review.

Wurtz.-A HISTORY OF CHEMICAL THEORY, from the Age of Lavoisier down to the present time. By AD. WURTz. Translated by Henry Watts, F.R.S. Crown 8vo. 6s.

"The discourse, as a résumé of chemical theory" and research, unites singular luminousness and grasp. A few judicious notes are added by the translator."-Pall Mill Gazette. "The treatment of the subject is admirable, and the translator has evidently done his duty most efficiently." - Westminster Review.

\section{WORKS IN PHYSIOLOGY, ANATOMY, AND MEDICAL WORKS GENERALLY.}

Allbutt ( $T$. C.) -ON THE USE OF THE OPHTHALMO. SCOPE in Diseases of the Nervous System and of the Kidneys; also in certain other General Disorders. By THOMAS CLIFFord Allbutr, M.A., M.D. Cantab., Physician to the Leeds General Infirmary, Lecturer on Practical Medicine, \&c. \&c. 8vo. I5s.

THE EFFECTS OF OVERWORK AND STRAIN ON THE HEART AND GREAT BLOOD-VESSELS. (Reprinted from St. George's Hospital Reports.) 2s. 6d.

Anderson.-ON THE TREATMENT OF DISEASES OF THE SKIN : with an Analysis of Eleven Thousand Consecutive Cases. By Dr. McCall Anderson, Professor of Practice of Medicine in Anderson's University, Physician to the Dispensary for Skin Diseases, \&c., Glasgow. Crown 8vo. cloth. 5 s.

Barwell.-THE CAUSES AND TREATMENT OF LATERAL CURVATURE OF THE SPINE. Enlarged from Lectures published in the Lancet. By Richard BARwell, F.R.C.S., Surgeon to and Lecturer on Anatomy at the Charing Cross Hospital. Second Edition. Crown Svo. 4s. $6 d$.

Corfield (Professor $\mathrm{V}$. H.)-A DIGEST OF FACTS RELATING TO THE TREATMENT AND UTILIZATION UF SElvage. By W. H. Corfield, M.A., B.A., Professor of Hygiene and Public Health at University College, London. 8vo. IOs. 6d. Second Edition, corrected and enlarged.

"Mr. Corfield's work is entitled to rank as a standard authority, no less than a convenient handbook, in all matters relating to servage."-Athenæum.

Elam (C.)-A Physician's PRoblems. By Charles ELAM, M.D., M.R.C.P. Crown 8vo. 9s.

Contents :- "Natural Heritage." "On Degeneration in Man." 
"On Moral and Criminal Epidemics." "Body v. Mind." "Illusions and Hallucinations." "On Somnambulism." "Reverie and Abstraction." "The book is one which all statesmen, magistrates, clergymen, medical men, and parents should study and inwardly digest." -Examiner.

Fox.-Works by Wilson Fox, M.D. Lond., F.R.C.P., F.R.S., IIolme Professor of Clinical Medicine, University College, London, Physician Extraordinary to her Majesty the Queen, Sc. :-

DISEASES OF THE STOMACH : being a new and revised Edition of "The Diagnosis and Treatment of The Varieties of Dyspepsia." 8vo. Ss. $6 d$.

ON THE ARTIFICIAL PRODUCTION OF TUBERCLE IN THE LOWER ANIMALS. With Coloured Plates. 4to. 5s. $6 d^{*}$ In this Lecture Dr. Fox describes in minute detail a large number of experiments made by him on guinea-pigs and rabbits for the purpose of inquiring into the origin of Tubercle by the agency of dirict irritation or by septic matters. The work is illustrated by thrie plates, containing a number of coloured illustrations from nature.

ON THE TREATMENT OF HYPERPYREXIA, as Illustrated in Acute Articular Rheumatism by means of the External Application of Cold. Svo. 2s. $6 d$.

The object of this work is to showe that the class of cases included under the title, and which have hitherto been invariably fatal, may, by the use of the cold bath, be brought to a favourable termination.

Flower (W. H.) - AN INTRODUCTION TO THE OSTE. OLOGY OF THE MAMMALIA. Being the substance of the Course of Lectures delivered at the Royal College of Surgeons of England in 1870. By IV. II. Flower, F.R.S., F.R.C.S., Hunterian Professor of Comparative Anatomy and Physiology. With numerous Illustrations. Globe 8vo. $7 s .6 d$.

Although the present work contains the substance of a Course of Lectures, the form has been changed, so as the better to adapt it as a handbook for students. Theoretical vicius have been almost entirely exchuded: and while it is impossible in a scicntific treatise to avoid the employment of technical terms, it has been the author's endeavour to use no more than absolutely necessary, and to exercise due care in selecting only those that seem most appropriate, or which have received the sanction of general adoption. With a very few exceptions the illustrations have been drawn cxpressly for this work from specimens in the Museum of the Royal College of Surgeons.

Galton (D.) - AN ADDRESS ON THE GENERAL PRINCIPLES WHICH SHOULD BE OBSERVED IN THE CONSTRUCTION OF HOSPITALS. Delivered to the British Medical Association at Leeds, July 1869. By Douglas Galton, C.B., F.R.S. Crown 8vo. 3s. 6 d.

"An admirable exposition of those conditions of structure which most conduce to cleanliness, cconomy, and convenience."-Times. 
Hood (Wharton).-ON BONE-SETTING (so called), and its Relation to the Treatment of Joints Crippled by Injury, Rheumatism, Inflammation, etc. etc. By WHARTON P. HOOD, M.D., M.R.C.S. Crown Svo. 4s. $6 d$.

The author for a period attended the London practice of the late Mr. Hutton, the famous and successful bone-setter, by whom he was initiated into the mystery of the art and practice. In the present work he gives a brief account of the salient features of a bonesetter's method of procedure in the treatment of damaged joints, of the results of that treatment, and of the class of cases in which he has seen it prove successful.

Humphry. - Works by G. M. Humphry, MI.D., F.R.S., Professor of Anatomy in the University of Cambridge, and Honorary Fellow of Downing College :-

THE HUMAN SKELETON (including the Joints). With 260 Illustrations, drawn from nature. Medium 8vo. 28 s.

OBSERVATIONS IN MYYOLOGY. Svo. 6s. '

This work includes the IIyology of Cryptobranch, Lepidosiren, Dog. Fish, Ceratodus, and Pseudopus Pallasil, with the Nerves of Cryptobranch and Lepidosiren and the Disposition of Muscles in Vertebrate Animals. The volume contains a large number of illustrations.

Huxley's Physiology.-See p. Ir, preceding.

Journal of Anatomy and Physiology.

Conducted by Professors Husphriy and Newton, and Mr. Clark of Cambridge, Professor TURNer of Edinburgh, and Dr. IVRIGHT of Dublin. Published twice a year. Old Series, Parts I. and II., price $7 s .6 d$. each. Vol. I. containing Parts I. and II., Royal Svo., I6s. New Series, Parts I. to IX. 6s, each, or yearly Vols. I2s, 6d. each.

Leishman.-A SYSTEM OF MIDIVIFERY, including the Diseases of Pregnancy and the Puerperal State. By William Leishman, M.D., Regins Professor of Midwifery in the University of Glasgow; Physician to the University Lying-in Hospital; Fellow and late Vice-President of the Obstetrical Society of London, etc. etc. Svo. Illustrated. 30s.

Lankester.-COMPARATIVE LONGEVITY IN MAN AND THE LOIVER ANIMALS. By E. Ray Lankester, B.A. Crown 8vo. 4s. 6 .

Maclaren.-TRAINING, IN THEORY AND PRACTICE.

By ARchibald Maclaren, the Gymnasium, Oxford. Second and Cheaper Edition, enlarged. Crown Svo. 6s. $6 d$.

"The philosophy of human health has seldom received so apt an ex. position."-Globe. "After all the nonsense that has been written about training, it is a comfort to get hold of a thoroughly sensible hook at last." - John Bull. 
Macpherson.-Works by John Macpirerson, M.D. :

THE BATHS AND WELLS OF EUROPE; Their Action and Uses. With Notices of Climatic Resorts and Diet Cures. With a Map. New Edition, revised and enlarged. Extra fcap. 8vo. 6s. 6 d.

This work is intended to supply information which will afford aid in the selection of such Spas as are suited for particular cases. It exhibits a sketch of the present condition of our knowledge on the subject of the operation of mineral waters, gathered from the author's personal observation, and from every other availabls source of information.

OUR BATHS AND WELLS : The Mineral Waters of the British Islands, with a List of Sea-bathing Places. Extra fcap. 8vo. pp. xv. 205. 3 s. 6 d.

Maudsley.-Works by Henry Maudsley, M.D., Professor of Medical Jurisprudence in University College, London :-

BODY AND MIND: An Inquiry into their Connection and Mutual Influence, specially in reference to Mental Disorders; being the Gulstonian Lectures for I870. Delivered before the Royal College of Physicians. New Edition, with Psychological Essays added. Crown 8vo. 6s. $6 d$.

\section{THE PHYSIOLOGY AND PATHOLOGY OF MIND.} Second Edition, Revised. 8vo. I6s.

Morgan.-UNIVERSITY OARS : Being a Critical Enquiry into the After-health of the Men who rowed in the Oxford and Cambridge Boat-Race, from the year I 829 to I 869 , based upon the personal experience of the Rowers themselves. By JoHn E. Morgan, M.D., M.A. Oxon., F.R.C.P., late Captain of the John + (Coll. Univ.)? Physician to the Manchester Royal Infirmary, author of "The Deterioration of Races," \&c. Crown 8vo. IOs. $6 d$.

"Dr. Morgan's book presents in a most admirable manner full and accurate statistics of the duration of life, and of the causes of death, of all the men who have rowed in Oxford and Cambridge boats from 1829 to $\mathbf{1} 869$, and also gives letters addressed to the author by nearly every individual of the number."-Daily News.

Munro.-THE SCIENCE AND ART OF NURSING THE SICK. By Aneas Munro, M.D. Crown 8vo. $75.6 d$.

Contents.-The Sick-room-Nurses and Nursing-Sick DietAppliances - Nursing in Childbed-Invalid Cookery. - The Medical Times says: "There is much in this work that the young practitioner will be glad to learn. It is just the book to put into the hands of any intelligent woman intending to qualify as a surse, and if more heads of families were famitiar with its teach. ing, it would save them much anxiuty and the doctor much unnecessary trouble." 
Pettigrew.-THE PHYSIOLOGY OF THE CIRCULATION IN PLANTS, IN THE LOWER ANIMALS, AND IN MAN. By J. Bell Pettigrew, M.D., F.R.S., \&c. Illustrated by 150 Woodcuts. 8vo. I2s.

Practitioner (The).-A Monthly Journal of Therapeutics and Public Health. Edited by Francis E. ANstie, M.D. Svo. Price Is. 6d. Half-yearly vols., Svo. cloth. IOs. $6 d$. each.

Radcliffe.-DYNAIIICS OF NERVE AND MUSCLE. By Charles Bland Radcliffe, M.D., F.R.C.P., Physician to the Westminster Hospital, and to the National Hospital for the Paralysed and Epileptic. Crown 8vo. 8s. 6\%.

Reynolds (J. R.)-A SYSTEM OF MEDICINE. Vol. I. Edited by J. Russell Reynolds, M.D., F.R.C.P. London. Second Edition. Svo. 25s.

"It is the best Cyclopadia of medicine of the time." - Medical Press. Part I. General Diseases, or Affections of the Whole System. $\$$ I. - Those determined by agents operating from without, such as the exanthemata, malarial diseases, and their allies. \& II.-Those determined by conditions existing within the body', such as Giout, R'heumatism, Rickets, etc. Part II. Local Diseases, or Affections of particular Systems. \& I.-Diseases of the Skin.

A Sistem OF MEDICINE. Vol. II. Second Edition. Svo. $25 s$.

Part II. Local Diseases (continued). \& I.-Diseases of the Norvous System. A. General Nervous Diseases. B. Partial Diseases of the Nervous System. 1. Diseases of the Head. 2. Diseases of the Spinal Column. 3. Diseases of the Nerves. \$ II.-Discases of the Digestive System. A. Diseases of the Stomach.

A SYSTEM OF MEDICINE. Vol. III. 8vo. 25 s.

Part II. Local Discases (continued). \&II. Diseases of the Digestive System (continued). B. Diseases of the Mouth. C. Diseases of the Fauces, Pharynx, and Esophagus: D. Diseases of the Intestines. E. Diseases of the Peritoneum. F. Discases of the Liver. G. Diseases of the Pancreas. \$ III.-Diseases of the Respiratory System. A. Diseases of the Larynx. B. Diseases of the Thoracic Organs.

Reynolds (O.)-SEWER GAS, AND HOW TO KEEP IT OUT OF HOUSES. A Handbook on House Drainage. By Osborne Reynolds, M.A., Professor of Engineering at Owens College, Manchester, Fellow of Queen's College, Cambridge. Second Edition. Crown 8vo. cloth. Is. $6 d$.

"Professor Reynolds' admirable pamphlet will a thousand times over repay its cost and the reader's most attentive perusal."-Mechanics' Magazine.

Rolleston.-THE HARVEIAN ORATION, I873. By GEORGE Rolleston, M.D., F.R.S., Linacre Professor of Anatomy and 
Physiology, and Fellow of Merton College, in the University of Oxford. Crown 8vo. 2s. 6d.

Seaton.-A HANDBOOK OF VACCINATION. By EDWARD C. Seaton, M.D., Medical Inspector to the Privy Council. Extra fcap. 8vo. 8s. $6 d$.

\section{WORKS ON MENTAL AND MORAL PHILOSOPHY, AND ALLIED SUBJECTS.}

\section{Aristotle.-AN INTRODUCTION TO ARISTOTLE'S} RHETORIC. With Analysis, Notes, and Appendices. By E. M. Cope, Trinity College, Cambridge. 8vo. I4s.

ARISTOTLE ON FALLACIES; OR, THE SOPHISTICI ELENCHI. With a Translation and Notes by Edward Poste, M.A., Fellow of Oriel College, Oxford. Svo. $8 s .6 d$.

"It will be an assistance to genuine snudents of Aristotle."-Guardian. "It is indeed a work of great skill."-Saturday Review.

Birks.-FIRST PRINCIPLES OF MORAL SCIENCE; Or, a First Course of Lectures delivered in the University of Cambridge. By the Rev. 'T. R. Birks, Professor of Moral Philosophy. Crown 8vo. 8s. $6 d$.

" "ihis work treats of three topics all preliminary to the direct exposi. tion of Moral Philosophy. These are the Certainty and Dignity of Moral Science, its Spiritual Geography, or relation to other main subjects of human thought, and its Formative Principles, or some elementary truths on which its whole development must depend.

Boole. - AN INVESTIGATION OF THE LAIVS OF THOUGHT, ON WHICH ARE FOUNDED THE MATHEMATICAL THEORIES OF LOGIC AND PROBABILITIES. By GEORge BOOLE, LL.D., Professor of Mathematics in the Queen's University, Ireland, \&c. 8vo. I4s. Butler (W. A.), Late Professor of Moral Philosophy in the University of Dublin :-

LECTURES ON THE HISTORY OF ANCIENT PHILO. SOPHY. Edited from the Author's MSS., with Notes, by William HePworti Thompson, M.A., Master of Trinity College, and Regius Professor of Greek in the University of Cambridge. New and Cheaper Edition. 8vo.

Calderwood.- - IVorks by the Rev. Henry Calderwood, M.A., LL.D., Professor of Moral Philosophy in the University of Edinburgh :-

PHILOSOPHY OF THE INFINITE: A Treatise on Man's Knowledge of the Infinite Being, in answer to Sir WV. Hamilton and Dr. Mansel. Cheaper Edition. 8vo. 7s. 6d. 
Calderwood.-continued.

"A book of great ability. . . w written in a clear style, and may be easily understood by even those who are not versed in such discussions."-British Quarterly Review.

A HANDBOOK OF MORAL PHILOSOPHY. Second Edition. Crown Svo. $6 s$.

"It is, we feel convinced, the best handbook on the subject, intellectually and morally, and does infinite credit to its author."-Standard. "A compact and useful work, going over a great deal of ground in a manner adapted to suggest and facilitate further study. . . . His book will be an assistance to many students outside his own University of Edinburgh."-Guardian. "We cannot too heartily recommend this excellent manual to all teachers who are anxious that the faith of their pupils should be strengthened by sound philosophy and substantial logic, and that their philosophy should be enlightened by the purest of all lights - that from heaven." John Bull.

Green (J. H.)-SPIRITUAL PHILOSOPHY : Founded on the Teaching of the late SAMUEL TAYLOR Coleridge. By the late Joseph Henry Green, F.R.S., D.C.L. Edited, with a Memoir of the Author's Life, by John Simon, F.R.S., Medical Officer of Her Majesty's Privy Council, and Surgeon to St. Thomas's Hospital. Two Vols. 8vo. 25 s.

Huxley (Professor.)-LAY SERMONS, ADDRESSES, AND REVIEIVS. See Physical Science Catalogue preceding.

Jevons. - Works by W. Stanley Jevons, M.A., Professor of Logic in Owens College, Manchester :-

THE SUBSTITUTION OF SIMILARS, the True Principle of Reasoning. Derived from a Modification of Aristotle's Dictum. Fcap. 8vo. 2s. 6d.

' 'Mrr. Fevons' book is very clear and intelligible, and quite worth consulting."-Guardian.

Maccoll.-THE GREEK SCEPTICS, from Pyrrho to Sextus. An Essay which obtained the Hare Prize in the year 1868. By Norman Maccoli, B.A., Scholar of Downing College, Cambridge. Crown 8 vo. 3s. $6 d$.

"Mr. Maccoll has produced a monograph which merit the gratitude of all students of philosophy. His style is clear and vigorous; he has mastered the authorities, and criticises them in a modest but independent spirit."-Pall Mall Gazette.

M'Cosh.-Works by JAmes M 'Cosh, LL.D., President of Princeton College, New Jersey, U.S.

"He certainly shows himself skilful in that application of logic to psychology, in that inductive science of the human mind which is 


\section{$\mathrm{M}$ 'C Cosh (J.)-continued.}

the fine side of English philosophy. His philosophy as a whole is worthy of attention." - Revue de Deux Mondes.

THE METHOD OF THE DIVINE GOVERNMENT, Physical and Moral. Tenth Edition. 8vo. IOs. 6d.

"This work is distinguished from other similar ones by its being based upon a thorough study of physical science, and an accurate knowledge of its present condition, and by its entering in a decper and more unfettered manner than its predecessors upon the discussion of the appropriate psychological, ethical, and theological questions. The author keeps aloof at once from the à priori idealism and dreaminess of German speculation since Schelling, and from the onesidedness and narrononess of the empiricism and positivism which have so prevailed in England."-Dr. Ulrici, in "Zeitschrift. für Philosophie."

THE INTUITIONS OF THE MIND. A New Edition. 8vo. coth. Ios. $6 d$.

"The undertaking to adjust the claims of the sensational and intuitional philosophies, and of the à posteriori and à priori methods, is accomplished in this work with a great amount of success." Westminster Review. "I value it for its large acquaintance with English Philosophy, which has not led him to neglect the great German works. I admire the moderation and clearness, as well as comprehensiveness, of the author's views." -Dr. Dörner, of Berlin.

AN EXAMINATION OF MR. J. S. MILL'S PHILOSOPHY: Being a Defence of Fundamental Truth. Crown 8vo. $7 s .6 \%$.

"Such a work sreatly needed to be done, and the author was the man to do it. This volume is important, not merely in reference to the vicus of Mr. Mill, but of the whole school of writers, past and present, British and Continental, he so ably represents."-Princeton Review.

THE LAWS OF DISCURSIVE THOUGHT: Being a Textbook of Formal Logic. Crown 8*o. $5 s$.

"The amount of summarized information which it contains is very great; and it is the only work on the very important subject writh which it deals. Never was such a work so much needed as in the present day."-London Quarterly Review.

CHRISTIANITY AND POSITIVISH : A Series of Lectures to the Times on Natural Theology and Apologetics. Crown 8vo. $7 s .6 d$.

Masson.-RECENT BRITISH PHILOSOPHY : A Review with Criticisms ; including some Comments on Mr. Mill's Answer to Sir William Itamilton. By I)Avid Masson, M.A., Professor of Rhetoric and English Literature in the University of Edinburgh. Crown 8vo. $6 s$. 
"We can nowhere point to a work which gives so clear an exposition of the course of philosophical speculation in Britain during the past century, or which indicates so instructively the mutual infuences of philosophic and scientific thought." - Fortnightly Review.

Maurice.-Works by the Rev. Frederick Denison Maurice, M.A., Professor of Moral Philosophy in the University of Cam. bridge. (For other Works by the same Author, see THEOLOGICAL Catalogue.)

SOCIAL MORALITY. Twenty-one Lectures delivered in the University of Cambridge. New and Cheaper Edition. Crown 8vo. Ios. 6 .

"Whilst reading it we are charmed by the freedom from exclusiveness and prejudice, the large charity, the loftiness of thought, the eagerness to recognize and appreciate whatever there is of real worth extant in the world, which animates it from one end to the other. We gain new thoughts and new ways of viewing things, even more, perhaps, from bcing brought for a time under the influence. of so noble and spiritual a mind."-Athenæum.

THE CONSCIENCE: Lectures on Casuistry, delivered in the University of Cambridge. New and Cheaper Edition. Crown Svo. 5 s.

The Saturday Review say's: "We rise from them with detestation of all that is selfish and mean, and with a living impression that there is such a thing as goodness after all."

MORAL AND METAPHYSICAL PHILOSOPHY. Vol. I. Ancient Philosophy and the First to the Thirteenth Centuries; Vol. II. the Fourteenth Century and the French Revolution, with a glimpse into the Nineteenth Century. New Edition and Pretace. 2 Vols. 8vo. 25 s.

This is an Edition in two volumes of Professor Maurice's History of Philosophy from the earliest perzod to the present time. It was formcrly scattered throughout a number of scparate volumes, and it is belioved that all adnirers of the author and all students of fhilosophy will welcome this compact Edition. In a long introduction to this Edition, in the form of a dialogue, Professor Maurice justifies some of his own peculiar views, and touches upon some of the most important topics of the time.

Murphy,-THE SCIENTIFIC BASES OF FAITH. By, JOSEPH JOHN MURPHy, Author of "Habit and Intelligence." 8 vo. I 4 s.

"The book is not without substantial value; the writer continues the work of the best apologists of the last century, it may be with less force and clearness, but still with commendable persiasiveness and tact; and with an intelligent feeling for the changed conditions of the problem." - Academy.

\section{Picton,-THE MYSTERY OF MATTER AND, OTHER}


ESSAYS. By J. Allanson Picton, Author of "New Theories and the Old Faith." Crown Svo. Ios. 6d.

Contents :- The Mystery of Matter-The Philosophy of Ignorance-The Antithesis of Faith and Sight-The Essential Nature of Religion-Christian Pantheism.

Thring (E., M.A.)-THOUGHTS ON LIFE-SCIENCE. By Edward Thring, M.A. (Benjamin Place), Head Master of Uppingham School. New Edition, enlarged and revised. Crown Svo. $7 s .6 d$.

Venn.-THE LOGIC OF CHANCE : An Essay on the Foundations and Province of the Theory of Probability, with especial reference to its application to Moral and Social Science. By JoHN Venn, M.A., Fellow of Gonville and Caius College, Cambridge. Fcap. 8vo. 7s. $6 d$. 
Every Thursday, price 4d.; Monthly Parts 7s. 4d. \& 7s, 8d,; Half-yearly Volumes 1.0s, 6d.

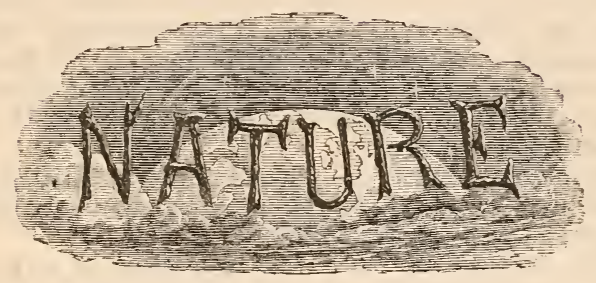

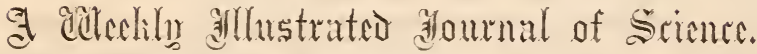

IT is now four years since NATURE first appeared, and the Publishers only state the acknowledged truth when they say that it has become the accredited organ of the leading scientific men in both the Old and the New Vorld.

No man can now lay claim to be considered intelligent and well-read unless he knows something of the principles of Science, and keeps himself au courant with the most recent developments of scientific principles in the various Arts. The all-important part which is being gradually assigned to Science in Education, in the Arts and Manufactures, in Commerce, and in Social Economy, is evident to all ; and there is no surer sign of a wide-spread regard for Science than the desire which exists among all classes of intelligent readers for scientific literature which is not beyond the range of those who can read and think. In the "fresh fields and pastures new," which scientific explorers are every year making accessible, are to be found feasts of instruction and pleasure of the highest kind, apparently inexhaustible, and fraught with the healthiest results to body and mind. So that, while "literature," in the old sense of the term, 
still holds, and will continue to hold, its place, there is a general craving abroad for scientific literature possessing a general interest.

One of the great aims of the Publishers of NATURE is to satisfy this laudable craving. This, however, is not accomplished by boiling down Science, or by offering to the public articles deprived of all that is characteristically scientific, but by avoiding the minute details of the separate sciences, and by expounding, in a popular and yet authentic manner, the GRAND RESUlTS OF SCIENTIFIC RESEARCH, discussing the most recent scientific discoveries, and pointing out the bearing of Science upon civilization and progress, and its claims to a more general recognition, as well as to a higher place in the educational system of the country.

ORIGINAL ARTICLES on all subjects coming within the domain of Science are contributed by the most eminent scientific men belonging to all parts of the world.

REviEWS, setting forth the nature and value of recent scientific works, are written for NATURE by men who are acknowledged masters in their particular departments.

The CORRESPONDENCE columns of NATURE, while forming a medium of scientific discussion and of intercommunication among the most distinguished men of Science, have become the recognised organ for announcing new discoveries and new illustrations of scientific principles among observers of Nature all the world over,-from Japan to San Francisco, from New Zealand to Iceland.

The SERIAL columus of NATURE contain the gist of the most important Papers that appear in the numerous Scientific Journals which are now published at home and abroad, in various languages; while longer ABSTRACTS are given of the more valuable Papers which appear in foreign Journals.

The PRINCIPAL SCIENTIFIC Societies and ACAdemies of the world, British and Foreign, have their transactions regularly recorded in NATURE, the Editor being in correspondence, for this purpose, with representatives of Societies in all parts of the world.

NoTES from the most trustworthy sources appear each week, recording the latest gossip of the scientific world at home and abroad.

In short, the Publishers venture to repeat, NATURE is the recognised organ of Science throughout the world. They have aimed, they believe successfully, so to conduct the paper that it shall have a claim on all readers. Its articles are brief and condensed, and are thus suited to the circumstances of an 
active and busy people, who have little time to read extended reviews and elaborate treatises.

NATURE is, moreover, well calculated to be of great service to teachers in any way connected with Science, or who give a place, however small, to Science in their course of instruction. It will keep them informed of all that is most recent and valuable in Science, and enable them to add constant freshness and interest to their instruction.

In order to make the paper more and more valuable to the general reader, and to supply a want which it is believed is felt by many, series of Papers, profusely illustrated, have been recently commenced, consisting of interestingly and instructively written articles, on particular scientific subjects - Scientific Discovery, Applications, History, Biography-by some of the most eminent scientific men in the kingdom. Among the works which are appearing, or will appear in NATURE shortly, the Publishers are already enabled to announce the following :-

"The Spectroscope and its Applications," by J. NoruIAN LOCKYER, F.R.S.

"The Origin and Metamorphoses of Insects," by Sir JoHN LUBBock, Bart., M.P., F.R.S.

"The Science of Weighing and Measuring", by H. W. CHISHOLi, Warden of the Standards.

"The Polarization of Light," by William SpotTiswoode, F.R.S.

"Meteorites," by N. S. Maskelyne, F.R.S., Keeper of the Mineral Department, British Museum.

"Mountain and Valley Sculpture," by Professor GEIkiE, F.R.S.

"The approaching Transits of Venus," by Professor Forbes.

"The Birth of Chemistry," by G. F. RodwelL, F.C.S. 
LONDON :

R. CLAY, SONS, AND TAYLOR, PRINTERS, BREAD STREET HILL. 




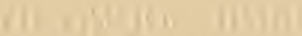

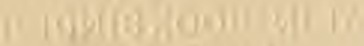

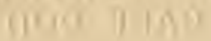




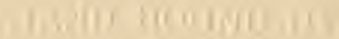

IN IN iJorsasmon is

X) 1;i (i) 1 

